LBL- -19215

DE85 010980

\title{
Angle-Resolved Photoemission Extended Fine Structure
}

\author{
John Joseph Barton \\ Ph.D. Thesis
}

\author{
Lawrence Berkeley Laboratory \\ University of California \\ Berkeley, California 94720
}

March 1985

\section{DISCLAIMER}

This report was prepared as an account of work sponsored by an agency of the United States Government. Neither the I'nited States Government nor any agency thereof, nor any of their employees, makes any warranty, express or implied, or assumes any legal liability or responsibility for the accuracy, completeness, or usefulness of any information, apparatus, product, or process disclosed, or represents that its use would not infringe privately owned rights. Reference herein to any specific commercial product, process, or service by trade name, trademark, manufacturer, or otherwise does not necessarily constitute or imply its endorsement, recommendation, or favoring by the United States Government or any agency thereof. The views and opinions of authors expressed herein do not necessarily state or reflect those of the United States Government or any agency thereof.

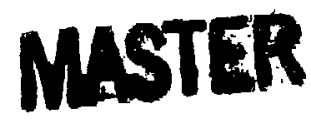


Abstract

Measurements of the Angle-Resolved Photoemission Extended Fine Structure (ARPEFS) from the $S(1 s)$ core level of $c(2 \times 2) S / N i(001)$ are analyzed to determine the spacing between the $S$ overlayer and the first and second $\mathrm{Ni}$ layers. ARPEFS is a type of photoelectron diffraction measurement in which the photoelectron kinetic energy is swept typically from 100 to $600 \mathrm{eV}$. By using this wide range of intermediate energies we add high precision and theoretical simplification to the advantages of the photoelectron diffraction technique for determining surface structures. We report developments in the theory of photoelectron scattering in the intermediate energy range, measurement of the experimental photoemission spectra, their reduction to ARPFES, and the surface structure determination from the ARPFES by combined Fourier and multiple-scattering analyses.

Curved-wavefront correstions to the single scattering of $l=1$ spherical waves are derived by applying lifting operators to the addition theorem for $\ell=0$ waves; the resulting formulation facilitates approximate calculation and physical interpretation because it contains a dominant term plus curved-wave corrections appearing as derivatives of the dominant term. A new addition theorem for translating spherical waves, $i^{l} h_{\ell}(k r) Y_{\ell m}(\hat{r})$, follows from a Taylor series expansion of the Fourier transform of these waves; the formula may also be interpreted as approximation of a rotation-translation- 
rotation sequence in which the magnetic quantum number expansion (MQNE) is truncated. This Taylor series - MQNE theorem is applied to the multiple scattering of photoelectrons in the context of a general discussion of small-atom approximations to electron scattering from non-overlapping potentials. We demonstrate that curved-wave corrections are large for forward scattering angles in the intermediate energy range even when the corrections for backscattering may be neglected; the Taylor series - MQNE allows stepwise addition of these corrections. Incorporating the new multiple-scattering method, we re-examine the theory of photoelectron scattering in the intermediate energy range arriving at a model which includes selected multiple scattering events, curved-wave corrections, analytic aperture Integration, anisotropic thermal averaging within a correlated Debye model, and an isotropic mean free path inelasic damping; we demonstrate that electron refraction and dynamic screening do not affect ARPEFS for metallic systems. We show by example that curvedwave forward focusing is a fundamental physical effect in photoelectron diffraction: neither single-scattering nor plane-wave treatments are adequate for ARPEFS.

Techniques for Fourier analysis of experimental ARPEFS spectra are explored with general guidelines for taper weighting function selection and adaptation of autoregressive linear prediction methods for Fourler analysis being reported. These Fourler techniques are 


\section{DEDICATION}

I dedicate this thesis to my parents, John M. and Louise M. Barton, for their vision, their love, and their support. 


\section{ACKNOWLEDGEMENTS}

I am indebted to several colleagues for their expert collaboration In the course of the research reported in this thesis. I thank in particular, Prof. David A. Shirley for his support and consultation on every aspect of the science reported here, Zahid Hussain for his patient tutoring in experimental physical chemistry, Charlie Bahr for his aid in the analysis and presentation of the data, Steve Robey for his help in the experimental measurements and the development of the theoretical treatment. Specifically, Chapters 2 through 8 report work in collaboration with D.A. Shirley, Chapter 5 was developed with S. Robey, and Chapter 7 contains contributions from C.Bahr, S.Robey, Z.Hussain, and Eberhardt Umbach. Numerous stimulating discussions with Chuck Fadley and S.Y. Dave Tong aided my work.

I also wish to thank Eberhardt Umbach for his help in learning the techniques of surface science, Dennis Trevor for sharing his knowledge of instrumentation, and Lenny Klebanoff for no good reason. I am grateful to wini Heppler for the polished crystals and the smoked turkey. The staff of the stanford Synchrotron Radiation Lab deserves my appreciation; special thanks to Katherine Cantwell, Robert Mayer, Harry Morales, Piero Pianetta, and Mike Rowan.

Thanks to Prof. T.C. MCGill for the right advice at the right time.

I happily acknowledge the help and friendship o: the Shirley research group members. In the Last Days of the preparation of this thesis, I was fortunate to have the enthusiastic help of Barbara Moriguchi.

For her help, for her frlendship. I send my love to cindy. 
CHAPTER 1: INTRODUCTION......................... 1

I. Introduction for the Non-Specialist............. 2

II. Introduction for the specialist............... 7

III. Outline............................ 16

References.............................. 21

Figure Captions.......................... 23

Figures................................... 24

CHAPTER 2: CURVED WAVEFRONT CORRECTIONS FOR PHOTOELECTRON

SCATTERING.............................. 26

I. Introduction......................... 27

II. Curved Wave Scattering of $\ell=1$ Photoelectrons......... 29

A. Plane waves.......................... 30

B. Spherical Waves...................... 33

II . Nature of Curved wave Corrections.............. 37

IV. Curved Wave Corrections to the Scattering from $\mathrm{Ni}$ Atoms 48

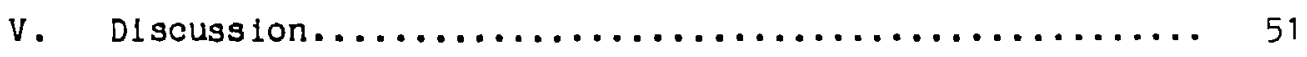

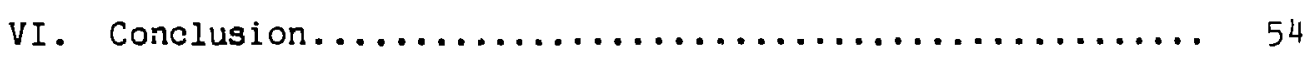

Appendix A: Alternative Derivation for Differential Form of OrIgIn-Shift Addition Theorem.............. 57

Appendix B: Application to EXAFS................. 60

References............................. 65

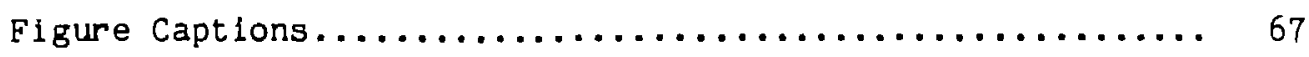


Figures.............................. 70

CHAPTER 3: APPROXIMATE TRANSLATION OF SCREENED SPHERICAL WAVES.. 84

I. Exact Origin-Shift Addition Theoren............. 85

II. Taylor Series Expansion..................... 88

III. Magnetic Quantum Number Expansion................ 94

IV. Conclusion............................ 97

Appendix A: Our Spherical harmonics................ 98

Appendix B: Coordinate Systems, Euler Angles, and Rotations. 99

Appendix $C:$ on the Taylor-Series Integrals............ 102

References.................................. 110

Figure Captions............................ 112

Figures................................... 113

CHAPTER 4: SMALL ATOM AFPROXIMATIONS FOR PHOTOELECTRON SCATTERING IN THE INTERMEdIATE ENERGY RANGE................. 114

I. Introduction......................... 116

II. Spherical wave Scattering and Nozawa's Gaunt Integral

Summation.................................. 121

III. Physically Motivated Small Atom Approximations........ 127

A. Point Scattering Model.................... 127

B. Homogeneous Wave Model.................... 130

C. Plane Wave Model....................... 133

D. Hybrid Model........................... 136

E. Formal and Practical Plane-Wave Limits.......... 137

IV. TS-MQNE Approximation..................... 140

V. The Taylor Serieg Scattering Factors.............. 148 
VI. Discussion.............................. 153

VII. Conclusion............................. 157

Appendix A: Comparing Notation with Ref.4............. 160

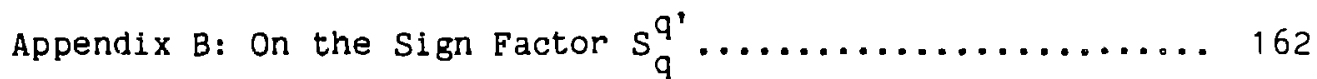

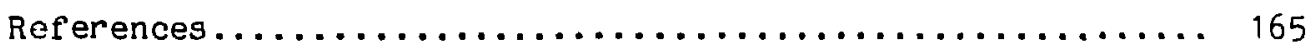

Figure Captions............................... 167

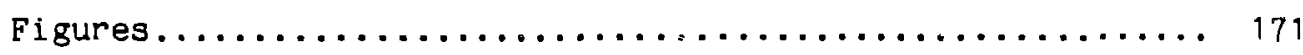

CHAPTER 5: THEORY OF ANGLE-RESOLVED PHOTOEMISSION EXTENDED FINE

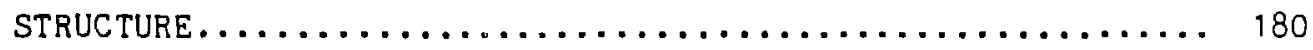

I. Introduction $\ldots \ldots \ldots \ldots \ldots \ldots \ldots \ldots \ldots \ldots \ldots \ldots \ldots, 182$

II. Goals of the Theory; The Model Systems............ 190

A. The Zero order Problem.................... 192

B. Connection to the Scattering Problem............ 193

C. The Scattering Problem.................... 194

III. The zero order Problem.................... 196

IV. The Scattering Problem..................... 209

v. Refraction and the Inner Potential.............. 214

VI. Flnite Angular Resolution................... 219

VII. Thermal Averaging........................ 224

VIII.Application to $\mathrm{c}(2 \times 2) \mathrm{S} / \mathrm{Ni}(001) \ldots \ldots \ldots \ldots \ldots \ldots \ldots . \ldots \ldots$

Ix. Conclusion................................ 247

Appendix A: Finite Angular Resolution................ 250

References.................................. 257

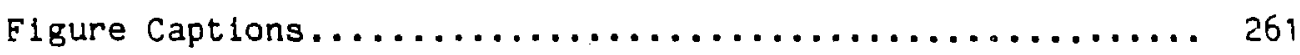

Figures...................................... 264 
CHAPTER 6: FOURIER ANALYSIS OF EXTENDED FINE STRUCTURE

WITH AUTOREGRESSIVE PREDICTION.................. 275

I. Introduction........................... 276

II. ARPEFS............................ 278

III. The Taper-and-Transform Method................ 280

IV. The Autoregressive Prediction Method.............. 284

V. Discussion............................... 291

VI. $\quad$ Summary.............................. 296

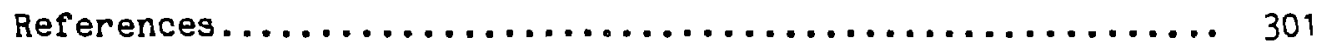

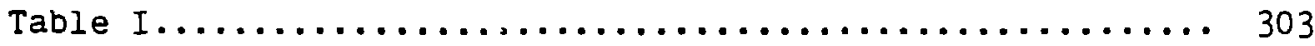

Figure Captions........................... 305

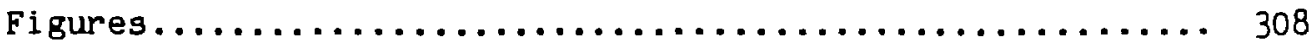

CHAPTER 7: THE MEASUREMENT AND ANALYSIS OF ARPEFS DATA:

APPLICATION TO C(2X2)S/NI(100)................ 316

I. Introduction.......................... 317

II. Angle-Resolved Photoemission.................. 319

A. Sample............................... 319

B. Electron Energy Analyzer.................. 320

C. Photon Monochromator..................... 320

D. Photoemission Measurements................. 321

E. Sources of Error........................ 321

III. The Extended Fine structure................. 325

A. Method................................ 325

B. Sources of Error...................... 330

IV. Extracting Geometry Information.............. 334

A. Fourier Transform...................... 334 
B. Multiple Scattering Analysis................ 339

C. Sources of Error...................... 345

v. Discussion and Conclusion.................. 348

References.................................. 350

Figure Captions............................. 352

Figures................................. 356

CHAPTER 8: A GENERALIZED RAMSAUER-TOWNSEND RESONANCE IN ARPEFS

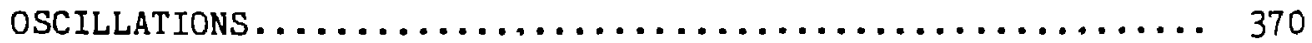

I. Introduction................................ 371

II. Experimental........................ 374

III. Generalized Ramsauer-Townsend Resonance........... 376

IV. Discussion............................ 381

v. Conclusion.............................. 385

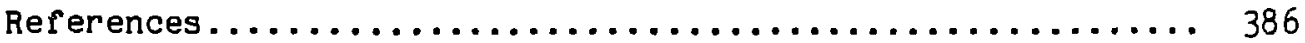

Elgure Captions............................ 388

Figures................................. 391

CHAPTER 9: CONCLUSION............................. 399 
CHAPTER 1:

INTRODUCTION

This thesis desoribes the development of a rew approach to determining the atomic geometry of surfaces. The method is based on the analysis of electron kinetic energy dependent oscillations in the partial cross section for core-level photoemission. The partial crosssection oscillations re observed with an angle-resolving photoelectron spectrometer; the oscillations are superimposed upon an atomic-like background and extend over the energy range from 50-1000 ev. Hence the title of this work: Angle-Resolved Photoemission Extended Fine Structure (ARPEFS). The acronym ARPEFS describes both the measured oscillations themselves and their use to determine surface structures. This thesis will Jiscuss a theory which quantitatively predicts the ARPEFS, and experimental measurements of $S(1 \mathrm{~s})$ ARPEFS from $c(2 \times 2) S / N i(100)$. The result is a structure determination for the well-known c(2x2)s/Ni(100) system by a new technique, a technique which has some unique qualifications as a tool for more sophisticated surface systems.

The remainder of this introduction is divided into three parts. In the first part--for the non-specialist--I will try to place this work within the whole body of chemistry and physics. The work of graduate students is necessarily obscure in detail and, to someone unfamiliar with the fields of surface structure determination or photoemission spectroscopy, this thes is solves an unfamiliar and thus uninteresting problem. The goal of the first part of this introduction is to outline the scientific interest in research like that reported here. In the second part--for the specialist--I will review previous work on partial 
cross-section oscillations similar to what is called ARPEFS here. The third part of the introduction contains an informal tour through the remaining chapters as a guide to those interested in only a particular aspect of this work.

\section{INTRODUCTION FOR THE NON-SPECIALIST}

The research reported here is a small contribution toward the eventual understanding of chemistry on surf'aces. Chemistry can occur when gases, liquid, or solid chemicals are mixed. When both constituents in a chemical reaction are in the same state--say $\mathrm{H}_{2}(g)$ and $\mathrm{O}_{2}(\mathrm{~g})$--and freely mix, then the reaction is called homogeneous. Almost all of classical chemistry is concerned with homogeneous chemistry, partly because-every molecule in such a reaction is equivalent: the mechanism of the chemical reaction can, in principle, be deduced by studying the individual reactants without regard to their position in space. The mechanism itself may require two molecules to meet along a particular axis, but any arrangement of two molecules will occur frequently when a large number of free molecules are mixed.

When the constituents in a reaction are in different phases--say the reduction of $\mathrm{H}_{2} \mathrm{O}$ at an electrode--the reaction is called heterogeneous. Surface chemistry is concerned with understanding heterogeneous chemistry, in large part, because the chemistry which can occur at the interface between two phases is so much different than that characteristic of a homogeneous reaction. Two of the most interesting differences are the constraints placed on the chemical reaction by the geometry of the interface, and the new dimension afforded by the "passive" participation of the bulk in a reaction occurring at an 
interface. When a molecule adsorbs to a solid surface, it is no longer free to reorient, and simultaneously its internal bond strengths are changed. Such a molecule can be much closer to the transition state for a reaction which would not occur without the surface. Or one part of the molecule may preferentially attach to a surface, placing adjacent parts in close proximity to the surface; the reactivity of the parts near the surface could be increased or decreased leading to selective reactions. These are differences in chemistry which have direct analogues in homogeneous chemistry, but when the passive role of the Interface is considered, very different chemistry can occur. The surface of a metal can (perhaps) serve as a reservoir for electrons or bonding orbitals, mediating a chemical reaction at a surface electronically in a manner similar to the thermal mediation provided by solvents in solution chemistry. A semiconductor, on the other hand, may mediate a photochemical reaction, absorbing the light energy and presenting it to a reactant molecule on the surface as a new, high. energy electronic state.

Surfaces are difficult to study for the same reasons that they are interesting. Foremost among the problems is ihe small ratio of surface atoms to bulk atoms: any physical measurement which is not preferentially sensitive to surfaces will have great difficulty examining only surface properties as distinct from bulk properties. Similarly it is sometimes difficult to distinguish if chemical reactions run for the purpose of studying a surface are characteristic of that surface or incidental surfaces or different, uninteresting sites on the same surface. Two approaches to this problem may be distinguished. In the first, chemical systems are devised which have a high ratio of 
surface area to volume, and techniques for studying bulk materials are then applied. This approaun has been especially fruitful for some special materials (e.g. exfoliated graphite) and for studying catalytic reactions which anyway occur on highly dispersed small particles. In the second approach, idealized, tightly controlled chemical systems are studied by techniques specifically chosen for their sensitivity to the surface region. This second approach provides a closer contact to idealized models of surface properties and allows a more detailed and elementary view of the surface chemical reaction. Furthermore, most of the idealized systems are solid single crystals providing an important and fruitful overlap with the results of solid state physics.

The work reported in this thesis falls in the second group and concerns new physical techniques for studying the surfaces of solid single crystals. It addresses perhaps the most fundamental question about an ideal surface: what is the arrangement of the atoms in the surface region of the crystal?. It achieves sensitivity to the surface by measuring electrons ejected into vacuum outside the crystal. This brings us to the ARPEFS measurement itself.

Briefly, our experiment consists of the following. To be specific we will describe the ARPEFS measurements of $c(2 \times 2) S / N i(100)$ analyzed here, but the same methods should apply to a variety of surface systems. We begin with a single crystal of $\mathrm{Ni}$ which is cut and polished into a disk $-1 / 4^{\prime \prime}$ in diameter and 1/16" thick with its surface parallel to the (001) crystal plane of $\mathrm{Ni}$. This crystal is mounted on a goniometer capable of rotating the crystal about two perpendicular axes through the polished surface and capable of heating the crystal to $1100^{\circ} \mathrm{C}$. The goniometer is mounted inside a stainless steel vacuum chamber equipped 
with an electron energy analyzer. The chamber is evacuated, and the crystal surface is cleaned until only $\mathrm{Ni}$ atoms can be detected. The crystal is then heated to heal any damage in the polished surface yielding a surface which has--on the atomic scale--large regions of ordered, crystalline, flat $\mathrm{Ni}(001)$ planes. This prepared crystal is then exposed to $\mathrm{H}_{2} \mathrm{~S}$ gas which decomposes to give an ordered, crystalline overlayer of sulfur atoms on top of tne $\mathrm{Ni}$ planes. This is the surface whose structure we wish to determine.

The crystal is lowered into a soft $x$-ray beam, and the electron analyzer is adjusted to collect only those electrons emitted from the crystal in a particular direction and coming from photoexcitation of the sulfur (1s) orbital. The number of those electrons is recorded, and the energy of the soft $x$-rays--and of the electron aralyzer--is increased. As the experiment proceeds, the number of electrons counted oscillates as the electron kinetic energy is increased: this is the angle-resolved photoemission extended fine structure (ARPEFS).

The physical explanation for the oscillations in photoemission intensity is similar to the more familiar description of $x$-ray diffraction. Both the ARPEFS oscillations and the $x$-ray diffraction spots are caused by interference between two (or more) waves which were at one point in phase but which have traveled different distances before arriving at the detector. If the difference in distance is an integral number of wavelengths-- $x$-ray wavelength for diffraction or de Broglie wavelengths for electrons--then the waves will again be in phase at the detector, and an $x$-ray spot or ARPEFS peak will be observed. If the difference in distance is not an integral number of wavelengths, the interference will not be purely constructive, and a lower intensity will 
be observed. The reason that both methods are useful for structure determination is that both $\mathrm{x}$-rays and electrons are directed into the Jetector by scattering from atomic cores. Thus the difference between two waves in distance traveled is directly related to the difference in distance between the atoms.

There are equally important dissimilarities between $x$-ray diffraction and ARPEFS. Foremost is the strength of the interaction between an electron and the atoms of a crystal compared to the interaction between $\mathrm{x}$-rays and those atoms. Electrons scatter very strongly and are absorbed by the crystal very readily so that only a few atomic layers are required to give a signal, and only a few atomic layers are close enough to the surface to contribute to the signal. $X$-rays scatter very weakly and are rarely absorbed so that many atomic: layers contribute to the $x$-ray diffraction. Thus while $x$-rays characterize the bulk, electrons characterize the surface region.

The ARPEFS physics and $x$-ray physics also differ in the reference point for the wave before it scatters into the detector. In $x$-ray diffraction, a collimated beam of $x$-rays is directed at the crystal sample: a plane perpendicular to the beam direction is the reference for scattering phase in $x$-ray diffraction. In ARPEFS, the reference point is the photoemitting $S$ atom, and the interference occurs primarily between the wave which travels directly from this $S$ atom to the detector and waves which scatter off nearby atom cores. Thus while $x-r a y$ diffraction provides a signal characteristic of th entire crystalline array illuminated by the beam, ARPEFS only contairs information about the local geometry about the $S$ atom. 
The distinction between local and extended information is quite important since the strong interaction that electrons have with the crystal must be understood in order to extract geometry information from the measurements. There is a more direct analogy to $x$-ray diffraction which is based on electrons--Low Energy Electron Dirfract 1 on--where a beam of electrons is sent into the crystal in place of $x$-rays. However Low Energy Electron Diffraction must attempt to extract the extended crystal information in the face of the electrons' strong interaction, a very diffjcult task. By concentrating on the information concerning the single emitting atom, ARPEFS has an easier job of extracting the geometry.

Armed with the experimental measurements and the physical basis we can proceed to develop a theory which connects the atomic geometries to the oscillations and use it to devise some scheme for using experimental oscillations to discover the atomic geometry. That is the contert of the following chapters.

\section{FOR THE SPECIALIST}

This second introduction reviews the work which inspired the investigation reported in the body of this thesis. The chapters themselves review material for their specific topics, and we will therefore cover only the larger picture here.

The basic physical phenomenon being exploited here for structure determination is the interference between the probability amplitude for a photoemitted electron to travel directly from the ionized atom to the detector and the probability amplitude for that electron to first 
scatter of a nearby atom. This phenomenon has been called a "finalstate interference ef fect" ${ }^{1,2}$ or photoelectron diffraction ${ }^{3}$ in previous work. While the first term is accurate, it is not very specific; the second term has come into wide spread use, and we must persist in its use as a generic label for the phenomenon despite its implied, misleading parallel to $x$-ray and low energy electron diffraction. We have coined a new term, ${ }^{4}$ Angle-Resolved Photoemission Extended Fine Structure (ARPEFS) to highlight several distinctive features of our new photoelectron diffraction technique. The name is entirely phenomenological in that it refers only to the observed oscillations rather t:an their physical interpretation. We do, however, intend for the ARPEFS to denote only those oscillations which have the same character as the oscillations in the extended energy range. In other words, there may be other final state interference effects particularly at low energy which cannot be described in the same fashion as ARPEFS and are not considered here.

The ARPEFS acronym does parallel Extended X-Ray Absorption Fine Structure (EXAFS) which is also a photoelectron inal-state interference effect. The EXAFS oscillations occur in the total $x$-ray absorption cross section, and hence they are an integral of the ARPEFS oscillations over all emission angles ${ }^{2}$ and overall final states excited at a particular $x$-ray energy. 5

The possibility of observing the diffraction of photoelectrons originating from adsorbate atoms, and of deriving structural information from this phenomenon, was suggested by A. Liebsch in 1974.' The effect was reported independently by three groups in $1978.3,6,7$ Normal photoelectron diffraction, (NPD), in which the photoelectron intensity 
was measured in the direction of the surface normal as a function of electron wave number $k$, has been used to determine a number of adsorbate geometries. $^{8,9}$ In NPD the interference-modulated intensity, $I(k)$, was compared to curves predicted by a LEED-like "quasikinematic" theory developed by S.Y. Tong and co-workers. ${ }^{10}$ The perpendicular distance $d_{\perp}$ between the adsorbate overlayer and the substrate is the parameter to which $I(k)$ is most sensitive, and NPD results were usually expressed in terms of $\mathrm{d}$

The NPD approach to surface structure determination yielded accurate structural parameters, but it suffered from two drawbacks. First, it depended upon an implicit theoretical analysis, much like LEED, albeit simpler. Second, it appeared to emphasize the single parameter $d_{\mathcal{L}}$, rather than showing approximately equal sensitivity to several structural parameters.

An apparently successful attempt ${ }^{11}$ was made to remedy the first deficiency, by using Fourler-transformation analysis. Several. peaks appeared in the Fourier transform of theoretical curves at distances near those predicted from interplanar spacings (in reality they actually corresponded to path-length differences, as discussed later).

The second problem was addressed by studying of $f$-normal $I(k)$ curves collected in directions normal to low Miller index planes that were not parallel to the crystal surface. The goal in these experiments was to perform a complete structure determination by Fourier transforming $I(k)$ data taken in several directions, thereby obtaining $d_{\perp}$ values relative to several planes. Initial attempts along these lines failed to yield any new structural information, ${ }^{12}$ but more recent experiments ${ }^{4}$ using sulfur-1s photoelectrons have succeeded beyond all expectations, leading 
to a new method for adsorbate structure determinations: angle-resolved photoemission extended fine structure (ARPEFS).

There are three major differences between NPD and ARPEFS. First, NPD concentrates on low kinetic energy photoelectrons, 30-150 eV, while ARPEFS works in the range 100-500 eV. In the lower range, NPD gains from the high photoemission cross section near threshold, but it suffers because the electron scattering is more isotropic and more sensitive to non-atomic details in the scattering potential. Although ARPEFS is more difficult to measure, it can be analyzed with simpler theory. The second difference between these diffraction methods is the energy range. For NPD, a large energy range is only modestly valuable in imfroving the confidence of structure assignment. For ARPEFS, the range must be as large as possible to give high-resolution Fourier transforms. The third difference is obvious: the emission angle in ARPEES is not necessarily selected to be normal. Normal emission can be a valuable choice for many surface geometries, but typically it should be only one of several directions measured.

In the course of this research, we have learned a great deal about the nature of the electron scattering physics which leads to the ARPEFS oscillations. To be confident of surface structure analysis with ARPEFS we must have a thorough understanding of the electron scattering. There is no large body of accurately known structures for adsorbate systems which we might use to calibrate an empirical model for ARPEFS; there is no analog to ARPEFS in the bulk of a material where known $x$-ray diffraction structures to be used for this calibration. Thus it seems that we must understand the electron scattering problem fundamentally. Thus a large fraction of this thesis is directed toward a deeper 
understanding of electron scattering in solids in the intermediate energy range. The detail of these chapters on theory must not obscure the underlying simplicity of the ARPEFS oscillations themselves. Our goal for ARPEFS is a surface technique capable of unambiguous, high accuracy $( \pm 0.02 \mathrm{~A})$ structure determination, and this will require both high precision experimental measurements and practical, high accuracy theoretical models. The final steps toward a highly accurate theory are the most painful ones, and this level of refinement is unnecessary for the design of experiments to measure ARPEFS. Thus we will review the single-scattering theory of ARFEFS of the basic phenomenon can be most simply grasped in this way.

Photoelectron diffraction is caused by the interference between direct photoelectron waves and waves which have scattered from ion cores surrounding the photoemitter. A simple treatment for the direct and scattered waves predicts the important physical effects. Fig. 1 illustrates the scattering geometry. We set our origin at the photoemitter and label each scattering center by an index "j". The bond vector $\vec{r}_{j}$ runs from the emitter to the scatterer; its length, $r_{j}=\left|\vec{r}_{j}\right|$, is the bond length. The photon polarization unit vector, $\hat{\varepsilon}$, makes an angle $B_{j}$ with the bond vector:

$$
\beta_{j}=\cos ^{-1} \frac{\hat{\varepsilon} \cdot \vec{r}_{j}}{\left|\vec{r}_{j}\right|}
$$

The scattering angle, $\theta_{j}$, is defined as the angle between the bond vector and the direction to the detector $\vec{R}$, 
$\theta_{j}=\cos ^{-1} \frac{\vec{r}_{j} \cdot \vec{R}}{\left|\vec{r}_{j}\right||\vec{R}|}$.

Figure 2 gives a schematic view of the scattering.

In our analyzer we measure a continuum wavefunction intensity $\psi_{\rho}^{*} \psi_{\hat{\rho}}^{*}$. This wave function contains contributions from a direct photoelectron wave, $\psi_{0}$, and a photoelectron wave scattered into the detector, $\psi_{j}$ :

$\psi_{f}=\psi_{o}+\sum_{j} \psi_{j}$

The direct wave for a 1 s core level is a spherical $p(b=1)$ wave:

$\psi_{0}=M(k) \cos \gamma \frac{e^{i k r}}{k r}$

Here the matrix element for photoemission, $M(k)$, serves as an uninteresting scale factor, while $Y$ measures the angle between the observation direction and the photon polarization direction.

This same wave also propagates into the substrate. Electrons with kinetic energies in the range 50-1000 eV scatter primarily from the highly localized ion core region. Thus a scattered wave appears to emanate from the position of the substrate atom at $\vec{r}_{j}$ :

$\psi_{j}-\frac{e^{i k\left|\vec{r}-\vec{r}_{j}\right|}}{k\left|\vec{r}-\vec{r}_{j}\right|}$. 
The scattered wave must be proportional to the amplitude of the original photoelectron wave in the region of the ion core. This amplitude is

$\psi_{0}\left(\vec{r}_{j}\right)=M(k) \cos \beta_{j} \frac{e^{1 k r_{j}}}{k r_{j}}$

where $\beta_{j}$ is the angle between direction $\vec{r}_{j}$ and the polarization vector and $r_{j}=\left|\vec{r}_{j}\right|$.

The outgoing scattered wave is also proportional to the scattering power $k\left|f\left(\partial_{j}, k\right)\right|$ of the ion core for the scattering angle, $\theta_{j}$, between the original propagation direction and the detector direction. The scattered wave is shifted in phase by a small amount $\phi_{j}$ by the potential of the ion core, giving finally

$\psi_{j}=M(k) \cos \beta_{j} \frac{e^{i\left(k r_{j}+\phi_{j}\right)}}{k r_{j}} k\left|f\left(\theta_{j}\right)\right| \frac{e^{i k\left|\vec{r}-\vec{r}_{j}\right|}}{k\left|\vec{r}-\vec{r}_{j}\right|}$

Near our detector the difference between a spherical wave at the origin and a spherical wave at $\vec{r}_{j}$ appears only as a phase shift equal to $-r_{j} \cos \theta_{j}$. (We can derive this by expanding the shifted spherical wave or by examining Fig. 2). Therefore we write the scattered wave at the detector (position $R$ ) as

$\psi_{j}=M(k) \cos \beta_{j} \frac{\left|f\left(\theta_{j}\right)\right|}{r_{j}} e^{i k\left(r_{j}-r_{j} \cos \theta_{j}\right)+\phi_{j} \frac{e^{i k R}}{k R}}$ 
Now we calculate, to first order, the ratio of the interference term to the direct term:

$x=\frac{I-I_{0}}{I_{0}}=\frac{\psi_{0}^{*} \psi_{0}+\sum_{j} \psi_{0}^{*} \psi_{j}+\sum_{j} \psi_{j}^{*} \psi_{0}-\psi_{0}^{*} \psi_{0}}{\psi_{0}^{*} \psi_{0}}$

$x=\sum_{j} \frac{\psi_{0}^{*} \psi_{j}+\psi_{j}^{*} \omega_{0}^{*}}{\psi_{0}^{*} \psi_{0}}$.

The direct term is simply:

$\psi_{0}^{*} \psi_{0}=M^{*}(k) M(k) \frac{\cos ^{2} \gamma}{k^{2} R^{2}}$

and the cross terms are

$2 R e\left\{M(k) M^{*}(k) \cos \gamma \cos B_{j} \frac{e^{1 k\left(r_{j}-r_{j} \cos \theta_{j}\right)+\phi_{j}}}{r_{j}} \frac{\left|f\left(\alpha_{j}\right)\right|}{k^{2} R^{2}}\right\}$

The cross section and $1 / R^{2}$ dependence are lost when the ratio is found:

$x(k)=2 \sum_{j} \frac{\cos \beta_{j}}{\cos \gamma} \frac{\left.\mid f \theta_{j}\right) \mid}{r_{j}} \cos \left[k\left(r_{j}-r_{j} \cos \theta_{j}\right)+\phi_{j}\right]$.

This is the single-scattering ARPEFS formula for $1 \mathrm{~s}$ photoemission. We will refer to the frequency of the entire argument of the cosine as the scattering path-length difference and to $\left(r_{j}-r_{j} \cos \theta_{j}\right)$ as the geometrical path-length difference. 
The general features of ARPEFS follows from this formula if we add that

i) $\left|f\left(\theta_{j}\right)\right|$ is peaked in the forward $\left(\theta_{j}-0\right)$ and backward $\left(\theta_{j}-180^{\circ}\right)$ directions, 13 (see figure 2),

1i) the phase $\phi_{j}$ is usually rearly constant as a function of $k$ with its linear component typically less than $10 \%$ of the geometrlcal path-length difference $\left(r_{j}-r_{j} \cos \theta_{j}\right)$,

iil) multiple scatterings are mostly forward fosusing, giving changes in amplitude and phase of the cosine but not in the geometrically important frequency, ${ }^{14}$ and

iv) exceptions to che usual behavior are due to properties of the scattering ion cores predictable in advance by studying the atomic ion core scattering amplitudes. 15

The important consequences of the basic model are that

i) atoms in the photoemission final state nodal plane will not produce ARPEFS oscillations, (see flgure 2),

ii) backscattering atoms give large oscillations,

iii) the osclllation amplitude decays with the inverse of the bond length,

iv) the oscillation frequency varies with scattering angle from a maximum of twice the bond length to, in principle, a zero frequency "oscillation" equivalent to forward focusing, and

v) the scattering phase must be lnown with moderate accuracy to connect the cosine frequency to the geometrical path-length difference.

The most straightforward way to take maximum advantage of this model would be to arrange the experimental geometry to maximize 
backscattering by positioning the angle-resolving electron analyzer opening and photon polarization vector in parallel and rotate the crystal to place substrate atoms behind the photoemitting adsorbate from the analyzer. To maximize the simplicity of the curves and the size of the ARPEFS, it is also advantageous to select a high symmetry emission direction if possible. These guidelines were followed for the experimental study of $c(2 \times 2) S / N 1(001)$ described in this thesis with one experimental geometry (normal emission) highlighting a secoñd layer $\mathrm{Ni}$ backscattering atom and having four-fold symmetry while the other experimental geometry ([110] emission) selects a first layer $\mathrm{Ni}$ near neighbor and has two-fold symmetry. A truly unknown system would require a preliminary ARPEFS measurement before the most appropriate geometry can be selected, and a low symmetry adsorption site may not favor particular emission direction choices, but the challenge of selecting the emission and polarization vector positions expresses the rich variety of experimental conditions avallable in the ARPEFS technique.

\section{OUTLINE}

This thesis is organized into nine chapters counting this introduction (Chapter 1) and the conclusion (Chapter 9). Each of the main chapters is self-contained in having its own specific introduction, central topic, and conclusion. When appropriate each chapter refers to the others, but the remainder of this overall introduction will be devoted to a more explicit description of their connections.

Chapters 2, 3, and 4 discuss aspects of the theory of wave scattering from potentials. Chapter 5 uses the results of the preceding chapters and some simple physical models of solids to construct a 
quantitative theory of ARPEFS. Chapter 6 explores the application of modern methods por frequency analysis for the purpose of directly displaying the scattering path-length differences by processing the data without intervening theory. The measurement of the angle-resolved photoemission spectra, the reduction of these spectra to ARPEFS curves, and the analysis of the oscillations with the methods of the preceding chapters are covered in Chapter 7 . Chapter 8 is devoted to a discussion by an unusual and interesting electron-scattering partial cross-section resonance observed in the normal emission ARPEFS from $c(2 \times 2) S / N i(001)$. Finally in Chapter 9, general conclusions are drawn primarily concerning the direction of Puture work.

Chapter 2 and Chapters 3 and 4 provide two separate but related solutions to a paradoxical problem encountered at the outset of this research. The problem is the physical description and numerical calculation of electron scattering in solids; the paradox is that the accuracy of very simple models of electron scattering improves as the energy increases in the intermediate energy range, but the calculation of electron scattering with more sophisticated models becomes increasing difficult. It would seem that some model must exist which lies between these extremes, providing accurate results over the whole range but becoming less expensive at the higher energy. Pursuing such a model led in two directions. Briefly, the simplest model for electron scattering in a solid treats the electron probability amplitude as a plane-wave in the region of each ion core, but in the sase of photoemission and multiple scattering, the actual waves come from a point source and thus are curved in the region of the ion core. Methods por incorporating the curved-wavefront effect exist, but they are too complex for use in the 
intermediate energy range. Chapter 2 develops a step-wise method for incorporating curved-wave corrections by starting with the basic isotropic curved wave and applying derivative lifting operators. The three dimensional derivatives required by these lifting operations are cumbersome, so in Chapter 3 a series expansion employing rotation matrices is derived. Insight from Chapters 2 and 3 are reformulated in Chapter 4 to give a reasonably general discussion of wavefront curvature models and to apply the expansion from Chapter 3 to multiple scattering of photoelectrons.

Chapter 5 incorporates the multiple-scattering equations from Chapter 4 into a framework for numerical simulation of the ARPEFS measurements including a discussion of the photoabsorption, transport of the photoelectron through the surface, thermal averaging, and aperture integration. The results are compared to the experimental curves as a progress check. This chapter would be a natural starting point for the experimentalist; the theorist may also wish to refer to Chapters 2 through 4 only after the significance of the curved-wave multiple scattering is evident. Furthermore the content of chapter 5 is somewhat more provocative in that several interesting theoretical and experimental questions are posed by our re-examination of electron scattering in the intermediate energy range.

Chapter 6 is a slight detour in preparation for the data analysis in Chapter 7. The cosine form for the ARPEFS oscillations suggests that one might Fourier analyze the experimental curves and observe peaks in the transform at the scattering path-length differences. Since the scattering path-length difference is dominated by the geometrical path length, such a Fourier analysis would provide a direct image of the 
surface structure information content of the ARPEFS measurement. Unfortunately, the practical energy range of the ARPEFS measurement limits the resolution of the Fourier spectrum so that individual Fourier peaks may correspond to more than one scattering atom. This is a wellknown problem of truncated Fourier series, and currently much active research in the digital signal processing field is devoted to improving. the resolution of Fourier spectra for well behaved signals. Chapter 6 applies some of the most recently developed methods of frequency analysis to the problem of extracting path-length differences from ARPEFS, but the results are only partially satisfying because in fact the ARPEFS signal is not always well behaved. The difficulty may be traced to the functional dependence of the factors in front of the cosine in the ARPEFS formula: if these factors combine to give an envelope whose Fourier transform is not sharply peaked and symmetric, then the Fourier transform of the ARPEFS curve will not peak at the path-length difference. Nevertheless, Prequency analysis is an important qualitative tool for ARPEFS analysis, and Chapter 6 provides a connection to a rapidly evolving fleld which might ultimately allow a direct analysis procedure to be developed.

Experimental measurements of the $S(1 s)$ ARPEFS from $c(2 \times 2) S / N 1(001)$ and their reduction to a surface structure determination are described in Chapter 7. Both the Fourier analysis methods of Chapter 6 and leastsquares fits of the numerical simulation method proposed in Chapter 5 are applied to deduce the (known) $\mathrm{S}-\mathrm{N} 1$ bond length and to suggest that the interlayer spacing between the first and second $\mathrm{Ni}$ layers is expanded from its bulk value by $-3 \%$. Sources of error for the complete procedure are discussed. 
Chapter 7 concentrates on features of the Fourler spectrum which do peak near scattering path-length differences. Chapter 8 reports the observation of an electron scattering partial cross-section resonance. The strong amplitude dependences which accompany this resonance modulate the ARPEFS oscillations and split the corresponding Fourier peak. The structure information can be extracted nevertheless, and the resulting S-NI bond length is in agreement with that derived in Chapter 7.

Finally, Chapter 9 concludes with a look to the future.. For every question resolved in this thesis, two or more are posed by the solution. While the power and experimentally feasible precision of ARPEFS as a technique for surface structure determination are eyident from previous studies of photoelectron diffraction combined with our new understanding of the advantages of the intermediate energy range, practical measurements with hlgh accuracy require $f$ urther work. The conclusion in Chapter 9 speculates on the directions that this future work might take. 


\section{REFERENCES}

1. A. Liebsch, Phys. Rev. Lett. 32, 1203 (1974).

2. P.A. Lee, Phys. Rev. B 13, 5261 (1976).

3. S.D. Kevan, D.H. Rosenblatt, D. Denley, B.-C. Lu, and D.A. Shirley, Phys. Rev. Lett. 41, 1565 (1978).

4. J.J. Barton, C.C. Bahr, Z. Hussain, S.W. Robey, J.G. Tobin, L.E. Klebanope, and D.A. Shirley, Phys. Rev. Lett. 51, 272 (1983).

5. J.J. Rehr, E.A. Stern, R.L. Martin, and E.R. Davidson, Phys. Rev. B $17,560(1978)$.

6. a) S. Kono, C.S. Fadley, N.F.T. Hall, and Z. Hussain, Phys. Rev. Lett. 41, 117 (1978); b) S. Kono, S.M. Goldberg, N.F.T. Hall, and C.S. Fadley, Phys. Rev. Lett. 41,1831 (1978).

7. D.P. Woodruff, D. Norman, B.W. Holland, N.V. Smith, H.H. Farrell, M.M. Traum, Phys. Rev. Lett. 41, 1130 (1978).

8. a) S.D. Kevan, D.H. Rosenblatt, D.R. Denley, B.-C. Lu, and D.A.Shirley, Phys. Rev. B 20, 4133 (1979); b) S.D. Kevan, J.G. Tobin, D.H. Rosenblatt, R.F. Davis, and D.A. Shirley, Phys. Rev. B 23, 493 (1981); c) S.D. Kevan, R.F. Davis, D.H. Rosenblatt, J.G. Tobin, M.G. Mason, D.A. Shirley, C.H. LI, and S.Y. Tong, Phys. Rev. Lett. 46, 1629 (1981); d) D.H. Rosentllatt, S.D. Kevan, J.G. Tobin, R.F. Davis, M.G. Mason, D.R. Denley, D.A. Shirley, Y. Huang, and S.Y. Tong, Phys. Rev. B 26, 1812 (1982).

9. D.H. Rosenblatt, J.G. Tobin, M.G. Mason, R.F. Davis, S.D. Kevan, D.A. Shirley, C.H. Li, and S.Y. Ton\&s, Phys. Rev. B 23, 3828 (1981).

10. a) S.Y. Tong, C.H. LI, and A.R. Lublnsky, Phys. Rev. Lett. 39,498 (1977); b) C.H. LI, A.R. Lubinsky, and S.Y. Tong, Phys. Rev. B 17, 3128 (1978). 
11. Z. Hussain, D.A. Shirley, C.H. Li, and S.Y. Tong, Proc. Natl. Acad. Sci. USA $78,5293(1981)$

12. D.H. Rosenblatt, S.D. Kevan, J.G. Tobin, R.F. Davis, M.G. Mason, D.A. Shirley, J.C. Tang, and S.Y. Tong, Phys. Rev. B 26, 3181 (1982).

13. P.J. Orders and C.S. Fadley, Phys. Rev. B 27, 781 (1983).

14. J.J. Barton, S.W. Robey, D.A. Shirley, "Theory of Angle-Resolved Photoemission Extended Fine Structuren, LBL-19324, and Chapter 5.

15. J.J. Barton and D.A. Shirley, "A Generalized Ramsauer-Townsend Resonance Observed in ARPEFS Oscillations", LBL-19325, and Chapter 8. 


\section{FIGURE CAPTIONS}

Figure 1 Cross-sectional view of a fcc crystal (001) surface showing the experimental geometry and illustrating the parameters of the scattering formula. The angle-resolving detector lies along the vector labeled $e^{-}$([011] direction in this example), the photon polarization vector is $\hat{\varepsilon}$. The angle between these two vectors is $\gamma$. The vector from the emitter to a scattering atom $j$ makes an angle $\beta_{j}$ with the polarization vector and an angle $\theta_{j}$ with the emission direction.

Figure 2. Illustration of the scattering process. Ir.e tirect wave has an angular distribution dependent upon the : iitial state (1s) and the photon polarization vector, $\hat{\varepsilon}$. The scattering from each atomic center depends upon its scattering angle $\theta_{j}$. The scattering amplitude calculated for $300 \mathrm{eV}$ is shown superimposed on the scattering atom. This amplitude is strongly peaked in the forward and backward directions. The geometrical path-length difference is also illustrated. 


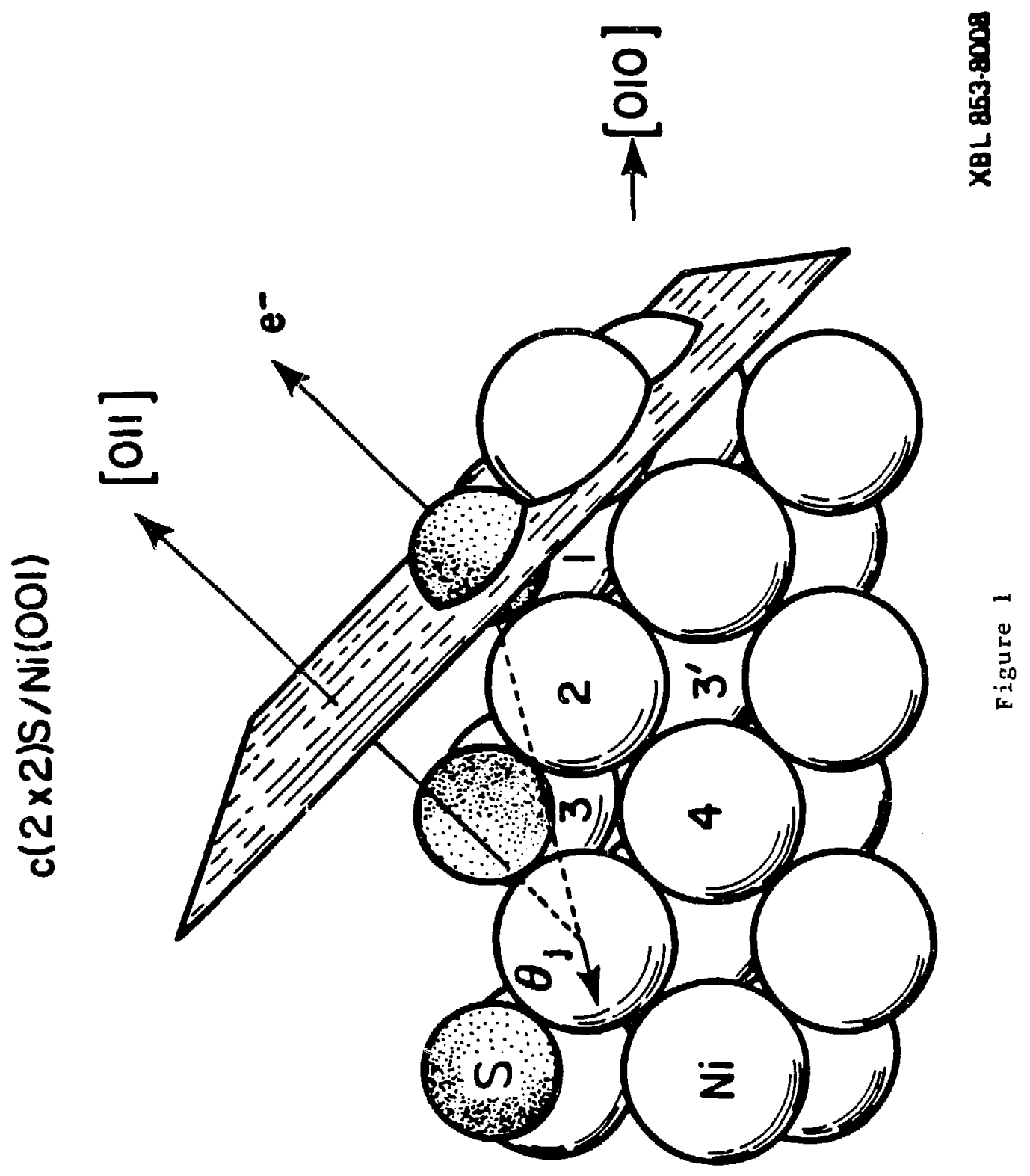




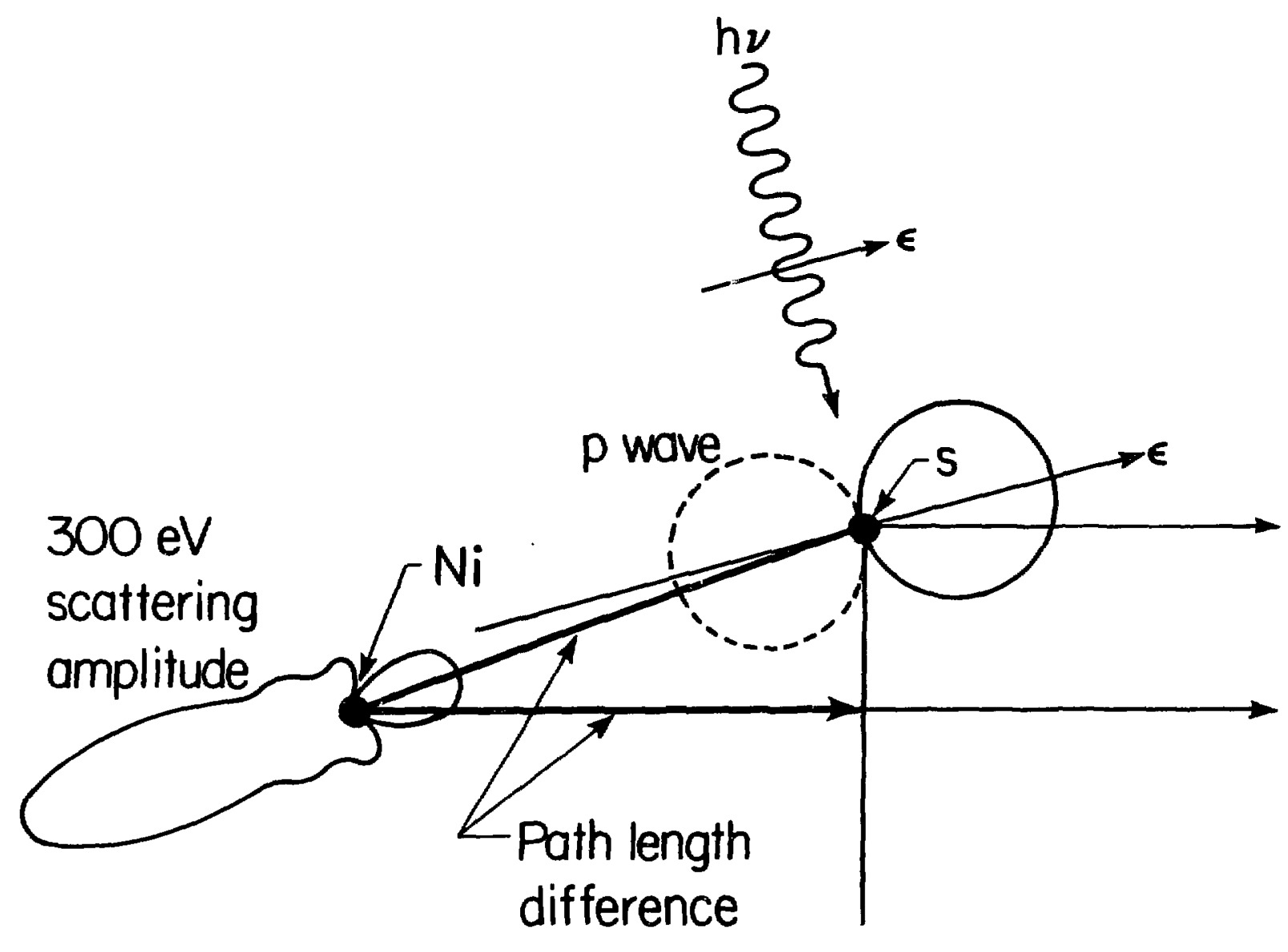

Detector

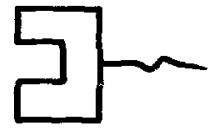

Figure 2 
CHAPTER 2:

CURVED WAVEFRONT CORRECTIONS FOR PHOTOELECTRON SCATTERING

ABSTRACT

We derive new, simplified formulas for the scattering of $l=1$ spherical waves from central potentials, as a basis for discussing curved wavefront corrections to single-scattering plane-wave models for Angle-Resolved Photoemission Extended Fine Structure (ARPEFS) and Extended X-ray Absorption Fine Structure (EXAFS). A differential form for the expansion of the screened spherical wave replaces the usual Gaunt integral form to facilitate the summation over equivalent magnetic sublevels in the scattered wave. Spherical wave scattering factors are defined and interpreted as corrections to the plane- eve scattering factor. We argue and demonstrate by example that the remarkable success of plane-wave models does not result from reaching the spherical wave asymptotic limit; instead successive partial wave corrections cancel for backscattering at high energy. The new scattering formulas allow curved-wavefront numerical calculations to be performed with little more effort than plane-wave formulas. 


\section{INTRODUCTION}

Understanding the motion of unbound electrons in solids is an interesting problem with important implications for surface structure determination methods based on electron scattering. The energy range from 20-200 eV has been studied extensively as a basis for the analysis of LOW Energy Electron Diffraction (LEED) data; ${ }^{1-3}$ more recent work in the energy range 20-1000 ev has been inspired by the explosive growth in the number of Extended X-ray Absorption Fine Structure (EXAFS)

measurements. 4 In the case of LEED, the incldent electron plane wave is simply described, but it excites every atom in the surface region, leading to a complex scattering problem; in the case of EXAFS only a single chemical element is excited by the $x$-ray beam, but the entire $x-$ ray absorption process must be understood and the observed modulations correspond to a special multiple scattering event. 5 Thus we suggest that an even more recerit technique, ${ }^{6}$ Angle-Resolved Photoemission Extended Fine Structure (ARPEFS) may be a more straightforward measurement for further understanding of electron scattering in the 50$1000 \mathrm{eV}$ range. ARPEFS measures partial cross-section oscillations of photollectrons; only electrons from a single chemical element are measured and a $4 \pi$ angular integration is not necessary. This paper investigates one aspect of the theory of electron scattering in solids, the role of curved wave corrections to the plane-wave single-scattering of (1s) photoelectrons.

A more practical motivation for this work is the interesting discrepancy between ARPEFS measurements and simple scattering theory results for the $c(2 \times 2) S / N i(100)$ system. Experimentally, a relatively simple Fourier transform spectrum led to the conclusion that only 
nearest neighbor and backscattering non-neighboring atoms contributed substantially to the observed spectrum. ${ }^{6}$ In other words, the number of important scattering atoms was small, permitting a simple interpretation of the Fourier spectrum. This conclusion has been recently challenged by Bullock, Fadley, and orders ${ }^{7}$ on the basis of single-scattering, plane-wave theoretical calculations. They demonstrated that a great many ion-cores should contribute to the theoretical curve under these and certain other approximations and hence no simple assignment of the Fourier peaks should be possible. Unfortunately, the reproduction of the experimental oscillations by these theoretical calculations is very poor, and we are lead to question the conclusions drawn from them.

To settle this issue, an improved theoretical calculation capable of matching the measured curves within experimental accuracy seems in order; if we know that the sum of the calculated scattering events is correct, then we can compare the relative intensity of these events with more confidence. The plane-wave single-scattering calculations may be Improved by:
1)
a more accurate atomic-like photoemission wavefunction (unscattered, direct wave),
1i) curved wave corrections,
1ii) multiple scattering,
iv) improved elastic scattering phase shifts, and
v) more accurate inelastic damping.

These Improvements are somewhat entwined, but in this paper we will concentrate on a single issue: when are curved wave (also called spherical wave) corrections important? 
We will examine only the simplest case of spherical wave scattering: single scattering of photoelectrons excited from a (1s) core level. We derive new formulas for this scattering in section II, applicable to both ARPEFS and EXAFS experiments. These formulas facilitate a qualitative discussion of curved wave corrections which occupies section III. In section IV we evaluate individual terms in these formulae for the example of a NI atom potential. Our discussion In section $V$ centers on possible generalizations to higher angular momenta. Finally, we address the impact our results might have on calculation of extended fine structure.

II. CURVED WAVE SCATTERING OF $\ell=1$ PHOTOELECTRONS

Our scattering system consists of a photoemitting atom and an array of non-overlapping Ion-core potentials. Zero-order calculation of the photoemission partial cross-section would ignore the lon-core array and only consider the atomic-like photoabsorption. Corrections caused by scattering from the ion core potentials gives the ARPEFS oscillations. Since we are only concerned with the oscillations, the details of zeroorder calculation are not relevant: we need only know the zero-order wave function. With dipole selection rules, polarized 11 ght, a (13) core-level initial state, and complete metallic screening the zero-order wave function is proportional to:

$\Psi_{0}(\vec{r})=\ln 1(k r) Y_{10}(\hat{r})$

Here $h_{\ell}(k r)$ is the spherical Hankel function of the first kind ${ }^{8}$ (we will omit the usual superscript (1) as in $h_{\ell}^{(1)}(k r)$ and we will not use 
spherical Hankel functions of the second $k i n d), Y_{\ell m}(\hat{r})$ is the spherical harmonic evaluated at the angles given by the unit vector, $\hat{r}$, in the direction of $\vec{r}$, and $k$ is the electron's wavenumber $f$ ar fror the photoemitter. Notice that we have selected the polarization vector of the light for our $\hat{z}$ axis to simplify the zero-order wave function description. The first-order corrections to this wave function are generated by including scattered waves emanating from each nearby ion core. The partial wave method ${ }^{9}$ for calculating these scattered waves has three steps:

1) expand the incident wave as an angular momentum series about the ion-core position,

11) multiply each "partial wave" in this series by a (complex) scattering amplitude (which also shifts the wave phase), 11i) sum the non-zero partial waves to give the full scattered wave.

It is the Pirst step which distinguishes plane wave from spherical wave scattering.

\section{A. PL,ANE haVes}

As a basis for our discussion of the curved wave effects we repeat the derivation of the plane wave ARPEFS model first presented by Lee, 5 but following more closely the method used by Lee and Pendry ${ }^{10}$ in their derivation of the EXAFS formula.

In a plane wave approximation, ${ }^{5}$ the photoelectron wave is represented near the scattering center by the value of the wave at the center, times a plane wave: 
$\psi_{0}(\vec{r})=\operatorname{ih}_{1}(k r)\left(\frac{3}{4 \pi}\right)^{1 / 2} \cos \theta_{\varepsilon a} e^{1 k \hat{a} \cdot(\vec{r}-\vec{a})}$

where $\theta_{\varepsilon a}$ is the angle between the electric vector $\hat{\varepsilon}$ and the bond vector $\vec{a}$. Since we have already ignored wavefront curvature with this approximation, we replace the Hankel function by its asymptotic limit,

$i^{l} h_{\ell}(k a)-\frac{e^{i k a}}{1 k a}$

and apply the well-known Bauer formula,

$e^{1 \vec{k} \cdot \vec{r}}=\sum_{\ell=0}^{\infty}(2 \ell+1) i^{\ell} j_{\ell}(k r) P_{\ell}\left(\cos \theta_{k r}\right)$

to expand the photoelectron wave around the scattering center:

$\psi_{0}(\vec{r})=\left(\frac{3}{4 \pi}\right)^{1 / 2} \cos \theta_{\varepsilon a} \frac{e^{1 k a}}{i k a} \sum_{\ell=0}^{\infty}(2 \ell+1) i^{\ell} j_{\ell}\left(k r^{\prime}\right) P_{\ell}\left(\cos \theta_{a r^{\prime}}\right)$

Here $J_{\ell}(k r)$ is the incoming spherical Bessel function, $P_{\ell}(\cos \theta)$ is the Legendre polynomial, and $\vec{r}^{\prime}=\vec{r}-\vec{a}$. The scattering angle, $\theta_{a r}$, is defined as the angle between the propagation vector for the incident plane wave, $k \hat{a}$, and the outgoing wave direction $\vec{r}^{\prime}$.

To construct the scattered wave, we multiply each incoming partial wave by

$T_{\ell}(k)=\frac{1}{2}\left(e^{21 \delta_{\ell}}-1\right)=1 \sin \delta_{\ell} e^{1 \delta_{\ell}}$ 
where $\delta_{\ell}(k)$ is the partial wave phase shift. Surming the new outgoing wave gives

$\psi_{\vec{a}}\left(r^{\prime}\right)=\left(\frac{3}{4 \pi}\right)^{1 / 2} \cos \theta_{\varepsilon a} \frac{e^{i k a}}{i k a} \sum_{\ell=0}^{\ell}(2 \ell+1) T_{\ell}(k) i^{\ell} h_{\ell}\left(k r^{\prime}\right) P_{\ell}\left(\cos \theta_{a r}\right)(7)$

The sum of $\ell$ may be stopped at $l_{\max }$ when all higher partial waves have negligible amplitude, $\left|\mathrm{T}_{\ell}(k)\right| \sim 0, \ell>\ell_{\max }$. At the angle resolved detector, located along $\vec{R}$, we may replace the outgoing spherical waves by their asymptotic limit. Then a scattering factor is defined by

$\mathrm{f}_{\mathrm{aR}}(k)=\frac{1}{\mathrm{ik}} \sum_{\ell=0}^{\ell \max }(2 \ell+1) \mathrm{T}_{\ell}(k) \mathrm{P}_{\ell}\left(\cos \theta_{\mathrm{aR}}\right)$

to $\mathrm{give}$ the scattered wave at the detector as

$\underset{\vec{a}}{\Psi_{\vec{R}}(\vec{R})}=\left(\frac{3}{4 \pi}\right)^{1 / 2} \cos \theta_{\varepsilon a} \frac{e^{i k|\vec{R}-\vec{a}|}}{1 k R} \frac{e^{1 k a}}{a} \rho_{a R}$

The factor $\exp (1 k|\vec{R}-\vec{a}|)$ corrects for the different origin of the scattered wave and for $|\vec{R}| \gg|\vec{a}|$ we have $|\vec{R}-\vec{a}|-|\vec{R}|-|\vec{a}| \cos \theta_{a R}$. The direct wave at the detector is

$\psi_{0}(\vec{R})=\left(\frac{3}{4 \pi}\right)^{1 / 2} \cos \theta_{\varepsilon R} \frac{e^{i k R}}{1 k R}$

and we calculate the ARPEFS oscillations due to a single atom as 
$\psi(k)=\frac{\left(\psi_{0}+\psi_{a}\right)^{*}\left(\psi_{0}+\psi_{a}\right)}{\psi_{0}^{*} \psi_{0}}=\frac{2\left|\rho_{a R}\right|}{a} \frac{\cos \theta_{\varepsilon a}}{\cos \theta_{\varepsilon R}} \cos \left[k a\left(1-\cos \theta_{a R}\right)+\phi_{a R}\right]$

where $\mathrm{f}_{\mathrm{aR}}(\mathrm{k})=\left|\mathrm{f}_{\mathrm{aR}}\right| \exp \left(1 \phi_{\mathrm{aR}}\right)$. This formula has been used to analyze experimental ARPEFS data in ref. 6.

\section{B. SPHERICAL WAVES}

For spherical waves, the angular momentum expansion in its usual form is much more complex: ${ }^{11}$

$\operatorname{in}_{1}(k r) Y_{10}(\hat{r})=\sum_{\ell " m^{n}} G_{10 \ell n^{n} m^{i^{\ell}}} j_{\ell "}\left(k r^{\prime}\right) Y_{\ell " m^{n}}\left(\hat{r}{ }^{\prime}\right)$

where $\vec{r}^{\prime}+\vec{a}=\vec{r}$. This formula is the basis for Lee and Pendry's curved wave EXAFS formula. ${ }^{10}$ To make physical arguments about the nature of curved wave corrections to the plane wave formula, we need a simpler form for this expansion, which we will refer to as an origin-shift addition theorem.

An alternative expansion for spherical waves may be derived most readily from Nozawa's or Iginal paper ${ }^{12}$ which describes expansions of "Helmholtz's Solid Harmonics", his term for the product of spherical Bessel functions anid spherical harmonics, which we will call "spherical waves". Nozawa demonstrated that the origin-shift addition theorem results when the raising operator for Helmholtz's Solid Harmonics, ${ }^{13}$

$I^{\ell} h_{\ell}(k r) P_{\ell}^{m}(\cos \theta) e^{i m \phi}=\left(\frac{-i}{k}\right)^{m}\left(\frac{\partial}{\partial x}+\frac{1 \partial}{\partial y}\right)^{m} P_{\ell}^{(m)}\left(\frac{-1}{k} \frac{\partial}{\partial z}\right) h_{0}(k r)$ 
is applied to the origin-shift addition theorem for $h_{0}(k r)$ :

$h_{0}(k r)=\sum_{\ell=0}^{\infty}(2 \ell+1) i^{\ell} j_{\ell}\left(k r^{\prime}\right) i^{\ell} h_{\ell}(k a) P_{\ell}\left(\cos \theta_{a r^{\prime}}\right)$

Here $P_{\ell}^{(m)}(-i \partial / k \partial z)$ is the operator obtained by using $(-i \partial / k \partial z)$ as the argument of the mth derivative of the Legendre polynomial of order $\ell$. As we shall see, this differential form for the expansion eliminates the need for magnetic quantum numbers for the outgoing scattered wave and leaves explicit the angle dependence hidden within $G_{\text {lm }}$ "m" above. For our particular case the raising operator formula is

$\operatorname{in}(k r) P_{1}(\cos \theta)=\left(-\frac{1}{k} \frac{\partial}{\partial z}\right) h_{0}(k r)$

and the origin-shift addition theorem becomes 14

$$
\begin{aligned}
& \ln ,(k r) P_{1}(\cos \theta): \sum_{\ell=0}^{\infty}(2 \ell+1) i^{\ell n} J_{\ell^{n}}\left(k r^{\prime}\right) h_{0}(k a) \\
& *\left[d_{1}(k a) P_{1}\left(\cos \theta_{\varepsilon a}\right) d_{\ell "}(k a) P_{\ell n}\left(\cos \theta_{a \mu^{\prime}}\right)\right. \\
& -i \cos \theta_{\varepsilon a} \frac{\partial d_{\ell n}(k a)}{\partial(k a)} P_{\ell "}\left(\cos \theta_{a n^{\prime}}\right) \\
& \left.-1 \frac{\left(\cos \theta_{\varepsilon r^{\prime}}-\cos \theta_{\varepsilon a} \cos \theta_{a r^{\prime}}\right)}{k a} d_{\ell \ell^{\prime \prime}}(k a) \frac{\partial P_{\ell n^{\prime}}\left(\cos \theta_{a r^{\prime}}\right)}{\partial\left(\cos \theta_{a r^{\prime}}\right)}\right\}
\end{aligned}
$$

We have introduced $d_{\ell "}(k a)$ to represent the polynomial part of the spherical Hankel function: 
$i^{\ell \prime} h_{\ell \prime \prime}(k a)=\frac{e^{i k a}}{i k a} d_{\ell \prime \prime}(k a)=h_{0}(k a) d_{\ell \prime \prime}(k a)$

Note that for large ka $\gg \ell^{\prime \prime}\left(\ell^{\prime \prime}+1\right), d_{\ell "}(k a)=1.0$, and that $d_{\ell}(k a)$ may be calculated by recursion: $d_{\ell+1}=d_{\ell-1}-d_{\ell}(2 \ell+1) / 1 k a$.

As before, the scattered wave may be calculated by multiplying each Incoming partial wave amplitude by $T_{\ell}(k)$ to generate an outgoing partial wave; each outgoing wave may be replaced by its asymptotic limit when the amplitude is calculated at the detector, position $\vec{R}$.

We invent a generalized scattering factor based on our origin-shift formula as

$\mathrm{f}_{\mathrm{aR}}^{\mathrm{nm}}=\frac{1}{1 k} \sum_{\ell^{n=0}}^{\ell}\left(2 \ell^{n}+1\right) \mathrm{T}_{\ell^{\prime \prime}}(k) \frac{\partial^{\mathrm{n}} \mathrm{d}_{\ell^{n}}(k a)}{\partial(k a)^{\mathrm{n}}} \frac{\partial^{\mathrm{m}} \mathrm{P}_{\ell^{\prime \prime}}\left(\cos \theta_{\mathrm{aR}}\right)}{\partial\left(\cos \theta_{\mathrm{aR}}\right)^{\mathrm{m}}}$

and the scattered wave is then

$$
\begin{aligned}
\Psi_{\vec{a}}(\vec{R}) & =\left(\frac{3}{4 \pi}\right)^{1 / 2} \frac{e^{i k|\vec{R}-\vec{a}|}}{i k R} \frac{e^{1 k a}}{a}\left\{d_{1}(k a) \cos \theta_{\varepsilon a} e_{a R}^{00}\right. \\
& \left.-i \cos \theta_{\varepsilon a} f_{a R}^{10}-\frac{1}{k a} f_{a R}^{01}\left[\cos \theta_{\varepsilon R}-\cos \theta_{\varepsilon a} \cos \theta_{a R}\right]\right\}
\end{aligned}
$$

If we label the factor within the braces $F_{S P H}=|F| \exp \left(i \phi_{S P H}\right)$ we parallel the plane wave construction of $y(k)$ to find

$$
x(k)=2 \frac{\left|F_{S P H}\right|}{a \cos \theta_{\varepsilon R}} \cos \left[k a\left(1-\cos \theta_{\mathrm{aR}}\right)+\phi_{\mathrm{SPH}}\right]
$$


Clearly, $F_{\text {sph }}$ determines both the amplitude and phase of the oscillations we will measure. As ka becomes large, the factors $\mathrm{f}_{\mathrm{aR}}^{10}$ and $f_{a R}^{01}(k a)$ fall to zero, $d_{\ell}(k a)$ becomes 1.0, $f_{a R}^{00}$ tends to $f_{a R}$, and we have $\mathrm{F}_{\mathrm{SPH}}+\mathrm{P}_{\mathrm{aR}} \cos \theta_{\varepsilon \mathrm{a}}$

Thus by studying $\mathrm{F}_{\mathrm{sph}}$ compared to $\mathrm{f}_{\mathrm{aR}}{ }^{\cos \theta_{\varepsilon a}}$ we can learn when curved wave corrections will influence the single scattering of photoelectrons. An alternative derivation for this formula is outlined in Appendix A.

The same method may also be applied to calculation of oscillations In the total absorption cross section, the Extended $x-r$ ay Absorption Fine Structure (EXAFS). Here the scattered wave must be projected back onto the direct wave at the absorbing atom: the oscillations are an interference at the photoemitter. The derivation for (1s) core levels and polarized light is given in Appendix B. If we call

$$
E_{S P H}=\frac{1}{1 k} \sum_{\ell=0}^{\ell}\left(2 \ell^{n+1}\right) T_{\ell n}(k)(-1)^{\ell^{n}}
$$

$$
*\left[\cos ^{2} \theta_{\varepsilon a}\left[d_{1}(k a) d_{\ell^{n}}(k a)-1 \frac{\partial d_{\ell^{n}}(k a)^{2}}{\partial(k a)}\right]-\sin ^{2} \theta_{\varepsilon a}\left[\frac{d_{\ell^{n}}(k a)}{k a}\right] \frac{\ell(\ell+1)}{2}\right\}
$$

then we compare $E_{S p h}$ to $\cos ^{2} \theta_{E a} \rho_{a R}(\pi)$ to examine curved wave corrections for EXAFS.

We might proceed directly to numerical applications of these formulae, but the qualitative success of the plane wave approximation 
suggests that some Inglght into eiectron scattering may be gained by examining the individual terms in these spherical wave formulas compared to results from a plane-wave model. We take up this topic in the next section.

III. NATURE OF THE CURVED WAVE CORRECTIONS

In this section we examine the formulas derived in the previous section for the exact single scattering of $\ell=1$ spherical waves. We know that the plane wave scattering model is substantially correct so we concentrate on differences caused by allowing for wavefront curvature. We begin this section with a brief examination of the mathematical reduction of the spherical wave formulas to their plane wave limit. This provides one method for studying curved wave effects, but to be more specific we might inquire about the importance of the fundamental spherical nature of the waves which is independent of angular momentum embodied in exp(ikr)/1kr - compared to additional curved wave corrections due to the particular incident angular momentum. We will demonstrate that each term in the differential form, eqn 19, corresponds to specific curved wave corrections. The first term gives the basic correction common to all angular momenta, the second term corrects for additional radial structure specific to the incident angular momentum, while the third term corresponds to additional angular character specific to angular momentum.

Our curved wave formulas approach the $\mathrm{plane}$ wave results whenever the spherical Hankel functions can be replaced by their asymptotic limits (eqn. 3). In our notation this is equivalent to replacing the 
polynomial part of the spherical Hankel function, $d_{\ell}(k a)$ by 1.0 in our Pormulas:

$$
d_{\ell}(k a)=\left[1-\frac{\ell(\ell+1)}{21 k a}+\cdots\right]-1.0
$$

Thus we must first discuss the size of $\ell(\ell+1) /(2 i k a)$. Notice that the angular momentum in this formula is the scattered wave angular momentum, not the dipole selection rule momentum from the photoabsorption.

The contribution of each partial wave to the final scattered wave is dictated by the partial wave amplitude (eqn. 6). For every wavenumber, $k$, there will be some angular momentum $\ell_{\max }$ beyond which all partial wave amplitudes may be neglected. With some oriterion for this cutoff we can define an equivalent range, $r_{0}$, for the scattering potential: ${ }^{15}$

$$
\ell_{\max }\left(l_{\max }+1\right)-\left(k r_{0}\right)^{2}
$$

In other words, the largest signiflcant partial wave climbs in proportion to $k$. The asymptotic criterion then reads

$$
\frac{k r_{0}}{2}\left(\frac{r_{0}}{a}\right) \ll 1
$$

for the last significant partial wave. By this analysis we conclude that the spherical Hankel function can be replaced by its limit only for large a $\gg r_{0}$; higher energy actually leads us away from the limit. Of course, as the number of partial waves increases, the impact of the 
largest angular momentum on the value of the scattering factor decreases. To properly assess this effect we should consider in detail the welght of each partial wave, but for a crude estimate assume equal weights. Then the contribution of the largest angular momentum decreases roughly like $\left(1 / \ell_{\max }\right)$, giving an asymptotic criterion for the sum as

$\frac{1}{2}\left(\frac{r_{0}}{a}\right) \ll 1$

We expect $r_{0}$ to be $=1 A$ and for $|\vec{a}|$ equal to the nearest neighbor distance, $r_{0} / 2 a=0.2$. Under these assumptions the curved wave effects are not too small; we turn to study the curved wave formulas for ARPEFS and EXAFS.

For photoelectron scattering we have

$F_{S P H}=d_{1}(k a) \cos \theta_{\varepsilon a} p_{a R}^{00}-1 \cdot \cos \theta_{\varepsilon a} p_{a R}^{10}$

$-1 \rho_{\mathrm{aR}}^{01}\left[\frac{\cos \theta_{\varepsilon \mathrm{R}}-\cos \theta_{\varepsilon a} \cos \theta_{\mathrm{aR}}}{k a}\right]$

The first term,

$d_{1}(k a) \cos \theta_{\varepsilon a} p_{a R}^{00}$

$=\left[1+\frac{1}{k a}\right] \frac{\cos \theta_{\varepsilon a}}{1 k} \sum_{\ell=0}^{\ell \max }(2 \ell+1) \mathrm{T}_{\ell}(k) \mathrm{d}_{\ell}(k a) \mathrm{P}_{\ell}\left(\cos \theta_{a R}\right)$ 
is the only one which survives in the asymptotic $11 \mathrm{mit}$, ka $\gg \ell(\ell+1)$. By examining the origin-shift addition theorem for $h_{0}(k r)$ (eqn 14) we can show that this first term corresponds to the single scaticering of an $\ell=0$ wave - the $\mathrm{f}_{\text {aR }}^{00}$ factor - multiplied by the $\ell=1$ wave components--the $d_{\ell}(k a) \cos \theta_{E a}$ part. The scattering factor, $f_{a R}^{00}$, differs from the plane wave counterpart, $f_{a R}$, only by including a weighting on partial waves, $d_{\ell}(k a)$, dependent on $k a$. Since $h_{0}(k a)=\exp (i k a) / i k a$, we can see that this welghting corrects the plane-wave scattering factor for the variation in the spherical wave over the finite size of the ion core potential due to ( $1 / 1 \mathrm{ka})$.

The second term,

$-i \cos \theta_{\varepsilon a} \mathrm{f}_{\mathrm{aR}}^{10}=\frac{-i \cos \theta_{\varepsilon a}}{i k} \sum_{\ell=0}^{\ell}(2 \ell+1) \mathrm{T}_{\ell}(k) \frac{\partial \mathrm{d}_{\ell}(k a)}{\partial(k a)} \mathrm{P}_{\ell}\left(\cos \theta_{\mathrm{aR}}\right)$

contains the derivative of the polynomial part of the spherical Hankel function. The expansion of $d_{l}$ in equation (23) gives the leading term In the derivative as

$\frac{\partial d_{\ell}(k a)}{\partial(k a)}-\frac{1}{1 k a} \frac{\ell(\ell+1)}{21 k a}$

Since ka - 10 in the ARPEFS energy range we can anticipate this spherical wave correction being much smaller than the difference between $\mathrm{f}_{\mathrm{aR}}^{\mathrm{DO}}$ and $\mathrm{f}_{\mathrm{aR}}$ : the factor $\ell(\ell+1) / 21 \mathrm{ka}$ represents the leading correction to the plane wave form and $f_{a f}$ is smaller by 1/ka. This term is literally the radial variation of $f_{a R}^{00}$ : it corrects the $S$ wave origin shift, given 
by $\mathrm{f}_{\mathrm{ak}}^{00}$ : : or the variation in $\mathrm{h}_{1}(\mathrm{ka})$ over the potential not already contained in $\mathrm{e}_{\mathrm{aR}}^{00}$.

The third term,

$\frac{-1}{\mathrm{ka}}\left(\cos \theta_{\varepsilon R}-\cos \theta_{\varepsilon a} \cos \theta_{a R}\right) \mathrm{f}_{\mathrm{aR}}^{01}=$

$-i \frac{\left(\cos \theta_{\varepsilon R}-\cos \theta_{\varepsilon a} \cos \theta_{a R}\right)}{(k a)(i k)} \sum_{\ell=0}^{\ell}(2 \ell+1) T_{\ell}(k) d_{\ell}(k a) \frac{\partial P_{\ell}\left(\cos \theta_{a R}\right)}{\partial\left(\cos \theta_{a R}\right)}$

contains both an unusual angular factor and a derivative with respect to $\cos \theta_{\text {aR: }}$. This term accounts for the variation in the spherical wave amplitude laterally across the width of the potential. We can use spherical trigonometry to rewrite this term in an instructive fashion. If we place three unit vectors in the directions or $\hat{\varepsilon}$, the polarization vector, $\vec{R}$, the emission vector, and $\vec{a}$, the bond vector, at a common origin, then the vector tips will define a spherical triangle on a unit sphere with sides $\theta_{\varepsilon R^{\prime}} \theta_{\varepsilon a}$, and $\theta_{a R}$. Observing this triangle along the vector $\vec{a}$ we see that

$\cos \theta_{\varepsilon R}-\cos \theta_{\varepsilon a} \cos \theta_{a R}=\sin \theta_{\varepsilon a} \sin \theta_{a R} \cos \phi_{\varepsilon a R}$

where $\phi_{\varepsilon a R}$ is the dihedral angle between $\vec{\varepsilon}$ and $\vec{R}$ through $\vec{a}$. Since the associated Legendre polynomials are defined

$P_{\ell}^{m}(\cos \theta)=\sin ^{m} \theta \frac{d^{m} P_{\ell}(\cos \theta)}{d(\cos \theta)^{m}} \quad 0 \leq m \leq \ell$ 
we can write

$-i \frac{\left(\cos \theta_{\varepsilon R}-\cos \theta_{\varepsilon a} \cos \theta_{a R}\right)}{k a} \rho_{a R}^{01}$

$$
=\frac{\sin \theta_{\varepsilon a} \cos \phi_{\varepsilon a R}}{1 k} \sum_{\ell=0}^{\ell}(2 \ell+1) T_{\ell}(k) d_{\ell}(k a) \frac{P_{\ell}^{1}\left(\cos \theta_{a R}\right)}{i k a}
$$

Thus this correction to the plane wave form reaches its maximum when the scattering potential is located in the nodal plane $\left(\cos \theta_{\varepsilon a}=0 ; \sin \theta_{\varepsilon a}\right.$ - 1) of the outgoing spherical wave. The maximum size of $P_{\ell}^{1}\left(\cos \theta_{\text {aR }}\right)$ is $(\ell+1) / 2$ but all of the partial waves do not reach this maximum for the same angle. Nevertheless we can roughly say that this third term will peak near $\theta_{a R}-20^{\circ}$, giving a curved wave correction approximately $\ell(\ell+1) / 2 k a$ smaller than the first term.

To recap our assignment of the terms in the differential spherical wave formula to speciflc curved wave corrections, we associate the first term - containing $\mathrm{f}_{\text {ap }}^{00}$ - with the fundamental, angular-momentumIndependent nature of the incident wave, the second term - containing $\mathrm{f}_{\mathrm{aR}}^{10}$ - with radial corrections dependent on angular momentum, and the third term - containing $\mathrm{f}_{\mathrm{aR}}^{01}$ - with angular corrections dependent on angular momentum. From this assigmment, we can expect significant curved wave corrections to the single-scattering ARPEFS formula when

1) the scattering potential is near a node in the incident wave angular distribution,

1i) the scattering angle is near $0^{\circ}$ (forward scattering), or 1ii) the scattering factor is near resonance. We now consider these cases in more detail. 
When the center of a scattering potential lies in a nodal surface of the incident wave, the plane wave model, eqn 11 , predicts no scattering. For $l=1$ incident waves, this geometry means $\theta_{\varepsilon a}=90^{\circ}$ and $\cos \theta_{\varepsilon a}=0$. Thus only the third term of the differential formula is nonzero and thus this third term represents the entire curved wave correction for this geometry. It is interesting to note that the usual experimental geometries ${ }^{6}$ for ARPEFS prevent this third term from producing its maximum effect. To maximize the measured photocurrent, the electron detector in the direction $\vec{R}$, is usually placed nearly parallel to $\vec{\varepsilon}\left(\theta_{\varepsilon R}-0^{\circ}\right)$. If an atom has $\theta_{\varepsilon a}-90^{\circ}$ so that $\sin \theta_{\varepsilon a}-1$, then the scattering angle, $\theta_{\mathrm{aR}}$, must also be $\sim 90^{\circ}$ for the scattered wave to enter the detector: for this experimental geometry the condition $\left(\theta_{\varepsilon a}-90^{\circ}, \theta_{a R}-20^{\circ}\right)$ will never be satisfled. Just the opposite must be true for the unusual experimental geometry adopted by Sinkovic et al., in a recent Azimuthal Photselectron Diffraction experiment ${ }^{16}$. They selected $\theta_{\varepsilon R}=72^{\circ}$ and measured electrons emitted $10^{\circ}$ from the surface: many of the important forward scattering atoms would have $\sin \theta_{\varepsilon a}>.5, \cos \phi_{\varepsilon a R}=1$, and $\theta_{a R}-20^{\circ}$. Thus their observation that plane-wave calculations gave poor agreement with experiment may reflect the neglected variation in wave amplitude across the scattering potential rather than multiple scattering effects. When the scattering angle is near $0^{\circ}$ we can get large curved-wave corrections strictly from the difference between the first term containing $\mathrm{f}_{\mathrm{aR}}^{00}$ and the plane wave $11 \mathrm{mit}$. To demonstate this we expand $d_{\ell}$ (ka) according to equation 23 , and subtract the asymptotic plane-wave part: 
$\left(\mathrm{f}_{\mathrm{aR}}^{00}-\mathrm{f}_{\mathrm{aR}}^{\mathrm{PLANE}}\right)=\frac{1}{1 \mathrm{k}} \sum_{\ell=0}^{\ell}(2 \ell+1) \mathrm{T}_{\ell}(k)\left[\frac{\ell(\ell+1)}{21 \mathrm{ma}}\right] \mathrm{P}_{\ell}\left(\cos \theta_{\mathrm{aR}}\right)$

The maximum difference will occur for forward scattering since $P_{\ell}(1)=1$ and all the partial wave corrections add. Conversely the minimum curved wave corrections should be expected for backscattering since $P_{2}(-1)=$ $(-1)^{l}$ and successive partial waves tend to cancel. This overall description should be most accurate for higher energles where the partial wave amplitudes, $T_{\ell}(k)$, have little structure.

When the full scattering factor approaches zero near a Generalized Ramsauer Townsend resonance ${ }^{17}$ we can expect the third case for large curved wave corrections. For special values of electron wavevector, $k$, and scattering angle, $\theta_{a R}$, the partial wave sum will be zero due to exact cancellation of all partial wave components. The particular pair of values $\left(k, \theta_{a R}\right)$ at which the scattering factor becomes zero will differ between the plane-wave and spherical-wave models as they weight the Individual partial waves differently. Thus analysis of scattering resonance data with a plane-wave model will give incorrect scattering angles and the observed resonance energy position will not be correctly given by plane wave calculations. While the first two circumstances leading curved wave effects discussed above invol ve only one or another of the terms in the formula, the resonance calculation will depend in detall on all three terms.

Curved wave corrections to the EXAFS formula are directly analogous to the corrections for photoelectron diffraction. Since the "detector" for EXAFS is the photoemitting atom, the curved wave effects are 
squared: our detector is not asymptotically far from the scattering atom. The first term of the spherical wave scattering factor

$$
\frac{\cos ^{2} \theta \varepsilon a}{1 k} \sum_{\ell=0}^{\ell}(2 \ell+1) T_{\ell}(k)(-1)^{\ell}\left[d_{1}(k a) d_{\ell}(k a)-1 \frac{\partial d_{\ell}(k a)}{\partial(k a)}{ }^{2}\right.
$$

has the same angular dependence as the plane wave model. This term contains both the basic radial correction for $1 / 1$ ka--the first factor inside the brackets--and the radial derivative correction. As discussed above, the radial derivative factor is usually much smaller than the s-wave origin-shift.

The second term of the EXAFS spherlcal wave amplitude factor has the opposite angular dependence ccmpared to a plane wave model:

$$
\frac{\sin ^{2} \theta_{\varepsilon a}}{1 k} \sum_{l=0}^{l}(2 \ell+1) T_{\ell}(k)(-1)^{\ell}\left[\frac{d_{\ell}(k a)^{2}}{k a}\right] \frac{\ell(\ell+1)}{2}
$$

This term corrects for variation in the incident wave amplitude scross the potential, primarily due to the node in the $p$ wave angular distribution. Thus for atoms along the nodal plane perpendicular to the electric vector, this term represents the error made by neglecting the angular structure in the photoelectron wave.

Typically EXAFS analysis is not concerned with relative scattering amplitude of individual atoms. Most of the measurable signal comes from nearest neighbor atoms, all of which contribute oscillations of the same frequency. The overall EXAFS amplitude is not simply given by the magnitude of the scattering amplitude ${ }^{18,19}$ and hence the spherical wave 
corrections to the magnitude are of little consequence. Rather it is the phase of the scattering factor that is central to the EXAFS analys is and high precision should require spherical wave correction; the weight of the individual partial waves in the scattering factor sum will otherwige be incorrect. Of course, practical EXAFS analysis does not rely on the accuracy of the theoretical scattering factor: empirical phase shifts are nearly always derlved from known model compounds.

An Important EXAFS technique which does rely on relative scattering amplitudes is the polarization dependence employed to deternine structures on surfaces. ${ }^{20}$ Here the overall amplitude for nearest nelghbors is measured for several orlentations of the polarization vector with respect to the crystalline sample axis. The results are usually fitted to the angular distribution predicted by a plane wave model-- $\cos ^{2} \theta_{\varepsilon a}$--and ignores spherical wave erfects. We would expect the largest curved wave correction when ka is small, i.e. low $Z$ elements having short bond lengths and in the lower energy region, and when we need accurate angular distribution calculations for small $\cos ^{2} \theta_{\varepsilon a}$. Looking back at the EXAFS formula we also find some insight into the success of the plane wave model. The leading correction to the plane wave amplitude is

$\Delta=\left[\frac{E_{S P H}}{\cos ^{2} \theta_{\varepsilon a}}-\mathrm{P}_{\mathrm{aR}}^{\mathrm{plane}}(\pi)\right]-\frac{1}{1 k} \sum_{\ell=0}^{\ell}(2 \ell+1) \mathrm{T}_{\ell}(k)(-1)^{\ell} \frac{\ell(\ell+1)}{\mathrm{ika}}$

This term is just twice the correction for backscattering ARPEFS, and, as we argued above, the successive terms tend to cancel. Furthermore, we can make a crude argument that the $k$ dependence--and hence the 
frequency shift--of this correction will be very small. The factor $\ell(\ell+1) /$ ika strongly favors high angular momentum waves. If we define an impact parameter $b=\ell / k$, we can take a semiclassical approximation for the phase shift: 21

$\delta_{\ell}(k)=\frac{z(b)}{k r_{0}}$

Here, $Z(b)$ is the unscreened nuclear charge within a sphere of radius $b$ around the scattering atom. For large $l$ we assume small phase shifts to write

$T_{\ell}(k)=1 \delta_{\ell}(k)=\frac{1 Z_{\ell}(b)}{k r_{0}}$

and since $\ell \gg 1$ we have

$\Delta-\sum_{\ell=0}^{\ell} \frac{2 \ell}{1 k} \frac{Z_{\ell}(b)}{k r_{0}}(-1)^{\ell} \frac{\ell^{2}}{k a}$

To get a series whose 1 imit does not depend on $k$ we invent a sampling radius

$$
r_{n}=\frac{l_{\max -n}}{k}
$$

which coincides with $\ell_{\max }=k r_{0}$ for $\ell_{\max } \gg 1$. Then 
$\Delta=\sum_{n=0}^{\ell} \frac{2 r_{n}^{3}}{1 r_{0}^{a}} z\left(r_{n}\right)(-1)^{\ell} \max ^{-n}$

Since the $r_{n}$ are constants of the potential, this spherical wave correction is roughly independent of $k$. Note that this argument requires the low angular momentum to be insignificant and we may not conclude that $\mathrm{f}_{\mathrm{aR}}^{00}$ or $\mathrm{f}_{\mathrm{aR}}$ are roughly independent of $\mathrm{k}$ by similar steps.

IV. CURVED WAVE CORRECTIONS TO THE SCATTERING FROM Ni ATOMS

Now we turn to some specific examples of curved wave effects in the scattering of electrons from $\mathrm{Ni}$ atoms. We will begin by examining the angle dependence of the scattering factors at $5 \mathrm{~A}^{-1}(95 \mathrm{eV})$ and at $10 \mathrm{~A}^{-1}$ $(381 \mathrm{ev})$, followed by their $k$ and $r$ dependences. For each case we will compare $\mathrm{f}_{\mathrm{aR}}^{00}$ to the plane-wave limit, $\mathrm{f}_{\mathrm{aR}}$. As the 1 ast example we calculate the effect of curved wave corrections to the polarization dependence in surfare EXAFS. In all these examples we take $|\vec{a}|=2.23 A$. Figure 1 compares the amplitudes of $\mathrm{f}_{\mathrm{aR}}^{00}$ and $\mathrm{f}_{\mathrm{aR}}$ for scattering angles from $0-180^{\circ}$ at a wavenumber of $5 \AA^{-1}$ (see eqn. 28). The general trend confirms our qualitative discussion in the previous section: the largest corrections are in the forward scattering directions. Figure 2 gives the amplitude of $\mathrm{f}_{\mathrm{ap}}^{10}$ (eqn. 29); note the dramatic reduction in magnitude. The angular structure of $\mathrm{f}_{\mathrm{aR}}^{10}$ is rather similar to $\mathrm{f}_{\mathrm{aR}}^{00}$.

The angular spherical wave correction, eqn. 31 , is plotted in figure 3 as $\left|\sin \theta_{\text {aR }} \mathrm{f}_{\text {aR }}^{01}\right|$ to emphasize the fact that this correction is zero for forward $\left(\theta_{a R}=0^{\circ}\right)$ and exactly backscattering $\left(\theta_{a R}=180^{\circ}\right)$. The overall scale is 20 percent of the scale in figure 7 , but recall 
that two additional angle factors, $\sin \theta_{\varepsilon a}$ and $\cos \cdot \phi_{\varepsilon a R}$ reduce this correction unless the scattering geometry is speclal.

We have constructed figures 4,5 , and 6 to parallel figures 1,2 , and 3 , respectively, except $k=10 \mathrm{~A}^{-1}$ for these new figures. All three comparisons demonstrate that the curved wave corrections are not much smaller at this higher energy, but the cancellation of successive angular momenta due to $P_{\ell}(\cos \theta)=(-1)^{\ell}$ is much more effective. Thus all the large scattering-angle $\left(\theta>90^{\circ}\right)$ amplitudes are quite accurate ( 5 percent) in the plane wave model, while the amplitude for scattering through $32^{\circ}$ is too high by more than a factor of 2 .

We can also compare the scattering factor phase by plotting the argument of the complex ratio $\mathrm{f}_{\mathrm{aR}}^{00} / \mathrm{f}_{\mathrm{aR}}, 1 . e$. their phase difference, as in Figure 7. For $k=5 A^{-1}$, the phase difference is roughly +0.5 radians; note that the two angles where the phase difference is not near +0.5 radians correspond to scattering angles with small scattering amplitudes, see figure 1. The curve for $k=10 A^{-1}$. has the same behavior although the shift is about half as large.

The $k$ dependence of these scattering factors is illustrated for $\theta_{a R}$ $=173^{\circ}$ in figure 8, $\theta_{a R}=0^{\circ}$ in figure 9, and $\theta_{a R}=127^{\circ}$ in figure 10. The backscattering geometry, figure 8, is the most important one for ARPEFS and, fortunately, the plane wave model is rather accurate. As we noted above, the angular curved wave correction is eliminated by $\sin \theta_{a R}$

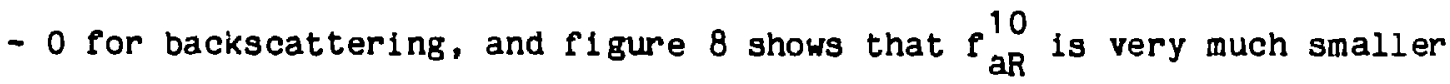
than $\mathrm{f}_{\mathrm{aR}}^{00}$. Thus $\mathrm{f}_{\text {aR }}^{00}$ by itself characterizes the backscattering of $l=1$ waves. Notice also that the plane wave amplitude error approaches a constant not equal to zero, for large $k$. This is explained in the same manner as the EXAFS discussion in the previous section. 
The greatest curved wave corrections occur in the forward directions; figure 9 gives the example of $\theta_{a R}=0^{\circ}$. The plane wave amplitude is roughly .2A too small over the entire range in $k$. Without the alternating sign of $P_{\ell}(-1)$ characteristic of backscattering, we see no approach to the plane wave limit at large $k$. Again $\mathrm{f}_{\text {aR }}^{10}$ is very small, at least a factor of 20 below $\mathrm{P}_{\mathrm{aR}}^{00}$; $\mathrm{P}_{\mathrm{aR}}^{01}$ cannot contribute to forward scattering as long as $\theta_{A R}<-10^{\circ}$. The phase difference (not plotted) between $\mathrm{f}^{00}$ and $\mathrm{f}_{\mathrm{aR}}^{\mathrm{plane}}$ is -.7 radians.

Finally, we consider scattering through $127^{\circ}$, the position of a Generalized Ramsauer Townsend resonance in $\mathrm{Ni}$. The resonance is a crossing of the origin in the complex plane by the complete scattering factor. The resonance position in energy and angle depends crucially on the cancellation of many partial waves and hence cannot be correctly predicted with a plane wave calculation. Figure 10 displays the scattering factors for $\theta_{a R}=127^{\circ}$. The factors $\left|\mathrm{f}_{\mathrm{aR}}^{00}\right|$ and $\left|\mathrm{f}^{\mathrm{plane}}\right|$ are reazonabiy close except in the resonance region near $8 \AA^{-1}$. The angular curved wave correction is now significant, especially since it conspires witt $\mathrm{f}_{\mathrm{af}}^{\mathrm{00}}$ to make the overall scattering amplitude zero at $k=7.5 \mathrm{~A}^{-1}$. $\left(f_{\mathrm{aR}}^{10}\right.$ has not been plotted; it is very small for $\left.\theta_{\mathrm{aR}}=127^{\circ}\right)$. The difference bstween exact single-scattering and plane-wave calculations is more dramatic in the phase of the scattering factor. Calculations done for values of ka corresponding to recent experimental measurements $^{17}$ are shown in figure 11. The phase jump at resonance is not correctly placed in angle or energy in the plane wave limit. To estimate the distance, $|\vec{a}|$, beyond which we can safely use the plane wave formula, we plot in figure 12 the radius at which $\mid f_{a R}^{00}-$ $f^{p l a n e} \mid \leq 0.06 A$, for two energies, $k=5 A^{-1}$ and $k=10 A^{-1}$. This 
criterion for the allowable error in scattering factor was chosen to be - 10 percent of the backscattering $\left(\theta_{\mathrm{aR}}-180^{\circ}\right)$ amplitude for $\mathrm{Ni}$. We see from this figure that plane wave calculations are never adequate by this oriterion for forward scattering at any energy or any practical radius. For angles greater than $45^{\circ}$, most scattering atoms within 10A of the photoemitter would require curved wave corrections in the lower energy region, while perhaps only the nearest neighbors require these extra calculations for $k=10 \mathrm{~A}^{-1}$.

To discuss an example calculation for the curved wave EXAFS formula, we adopt the second form for $x(k)$ given in Appendi $x$, eqn. (B13). Figure 13 compares $f^{1 s 0}$ to its asymptotic limit $f^{p l a n e}(\pi)$ and to $\mathrm{f}^{\text {an }}$. We see a close analogy between $\mathrm{f}^{\mathrm{iso}}$ for EXAFS and $\mathrm{f}_{\mathrm{aR}}^{00}$ for ARPEFS, but the curved wave corrections are larger for EXAFS (compare figure 8) since the "detector" is not asymptotically far from the scattering atom. Once again the large $k$ region approaches a non-zero constant plane-wave error. Perhaps most interesting, $\left|f^{\text {an }}\right|$ is seen to be nearly two orders of magnitude smaller than $\left|f^{1 s 0}\right|$ in this energy range. Thus, at least for $\mathrm{Ni}$ atoms, the standard EXAFS formula with $\mathrm{f}^{1 \mathrm{so}}$ replacing $\mathrm{f}^{\mathrm{plane}}(\pi)$ would give 1 percent accurate curved wave results. Furthermore, since the polarization dependence technique relies only on the assumption that the oscillations are proportional to $\cos ^{2} \theta_{\varepsilon a}$, curved wave corrections to the calculated amplitude ratios are entirely insignificant.

\section{v. DISCUSSION}

We have derived reis formulae and given examples for the curved wave scattering of $\ell=1$ spherical waves. What can we expect for more general spherical waves? We offer some qualitative ideas in this section. 
We envision two important cases: i) photoabsorption by $p, d$, and $f$ core levels giving spherical waves with higher angular momenta and magnetic sublevel occupations; and il) multiple scattering preceded either by photoelectron scattering or plane wave scattering typical for the LEED experiment. Both of these problems can be approached by the method we use here for $\ell=1$ waves. That is, the origin-shift addition theorem summed over magnetic sublevels can be differentiated to higher and higher order. The resulting expressions will be formidable so we will be content with estimates for now.

First we consider higher $\ell$ waves populated by photoemission. For core orbital initial states with $p, d$, or $f$ orbital angular momentum, two partial waves with $\ell \pm 1$ will be created. Each partial wave may be treated by the method of section II. We should always get a first term like $d_{\ell}(k a) Y_{\ell m}(a) f_{a R}^{00}$, the amplitude of the $\ell$ spherical wave times the scattering factor for $\ell=0$ waves. This is the only curved wave factor which survives the asymptotic limit and hence will always be the most important. Our discussion for $\ell=1$ virtually ignores $d_{\ell}(k a)$ as being close to 1.0 , but for higher angular momenta this factor may be important. Otherwise, this first term will follow the trends discussed in the previous section.

We should also always get curved wave corrections due to differences between the $(1 / \mathrm{ikr})$ dependence of $\mathrm{h}_{0}(\mathrm{kr})$ and the angularmomentum-dependent radial wave character through the potential region corrections analogous to $\mathrm{f}_{\mathrm{aR}}^{10}$. For higher angular momenta, the difference between the radial character of the incident spherical wave and the radial character of $h_{0}(k a)$ already included in the first term will increase. We might conclude from our $\mathrm{N} i$ example calculations that 
these radial variations are negligible for $\ell=1$ waves; for some higher $\ell$ we will be forced to include this term.

For all 2 , the radial variations should be less than the angular variations simply because spherical waves (except $\ell=0$ ) have stronger angle dependence. Thus curved wave corrections analogous to $f_{\text {af }}^{01}$ will be Increasingly important for higher angular momenta. These angular corrections are always greatest near nodes in the incoming wave, where the wave amplitude is changing most rapidly. The nodal regions has the least amplitude and the finite extent of the potential is anyway averaging opposite phase waves across the nodal surface, smoothing out the nodal structure. Hence, on the average, even these angular corrections will not be large. The phrase "on the average" is connected with the additional angilar vectors like $\sin \theta_{\varepsilon a} \sin \theta_{a R} \cos \phi_{\varepsilon a R}$ which multiply the curved wave angular correcticn.

In addition to more si gnificant curved wave corrections of the same type as the $2=1$ wave, higher angular momenta waves should also have corrections corresponding to higher order derivatives. Thus the second derivatives of the incoming wave across the extent of the potential will become important for some high $\ell$. Actual calculations are necessary to determine how important these corrections will be.

This leads us to the second important case, multiple scattering. While photoabsorption can populate only dipole allowed angular momenta, an outgoing scattered wave contains all angular momenta up to $l_{\max }$ $\mathrm{kr}_{0}$. To apply the method of this paper to the exact multiple scattering of spherical waves would--as a practical matter--require automation of the derivative calculations, a dubious improvement over the Gaunt integral summation formula, equation 12 . On the other hand, the 
outgoing scattered wave is no more than a spherical wave with an angular dependence and phase determined by scattering rather than by photoabsorption. Thus, approximate multiple scattering could be calculated by starting with $\mathrm{P}_{\mathrm{aR}}^{00}$ times the single scattered wave amplitude at the second scattering center and adding curved wave corrections by numerical differentiation of the single-scattered wavefunction.

VI. CONCLUSION

To summarize our work, we have

1) derived new curved wave formulae for single scattering of (1s) core level photoelectrons, appropriate for ARPEFS and EXAFS experiments,

1i) interpreted the individual scattering factors in this formula as different types of curved-wave corrections, allowing some guidelines to be devised to predict which scattering problems require curved-wave formulas,

1ii) given some idea of the size of these factors for $\mathrm{Ni}$ atom scattering, and

iv) discussed the possible generalization to higher angular momenta core levels.

The significance of these results is partly formal and partly practical. The remarkable accuracy of the plane wave model has been widely recognized, 4,22 but of ten attributed to the asymptotic limit of the spherical wave. Our new formulas more clearly demonstrate the origin of this convenience: the improved cancellation of partial waves 
at large $k$. Thus the accuracy of the plane wave model does not fmprove for large $k$ in forward scattering directions. This point way also be made by a semi-classical argument. As figure 14111 ustrates, forward scattering corresponds to large classical impact parameters; 21 backscattering corresponds to low enough impact parameter to sample the strong attractive center region of the potential. The wavefront curvature corrections are thus much larger for forward scattering directions which sample the extreme edges of the potential.

On the practical side, our new curved-wave formulas are scarcely more complicated than the plane-wave versions. Some advantage way also be made of the different angle dependences of each scattering factor, to minimize numerical computations. Hopefully, our qualitative discussion and numerical example will serve as some guide to estimate when curved wave effects may be important. Finally, we have demonstrated that curved wave EXAFS calculations can be quite accurate with only a minor modification of the plane-wave formula, a result which extends the recent work of Schal $\mathrm{ch}^{23}$ and of Gurman, et al. 24

Unfortunately, it is also clear from our results that curved wave effects cannot explain the difference between ARPEFS experiments and the single-scattering plane-wave calculations of Bullock, Fadiey, and orders. ${ }^{7}$ The curved wave corrections are typically - 20 percent and only that large in the forward directions. Thus while we have reduced the computational barrier to using curved wave calculation for ARPEFS, we can also conclude that the major discrepancies between theory and experiment are not due to curved wave corrections at least for single backscattering. 
We can characterize the disagreement between model calculations and ARPEFS measurement by ncting that scatterings from nearest neighbors and backscatterings from non- nearest neighbors appear to be much more dominant than predicted theoretically. Wavefront curvature increases scattering for some angles, decreases it for others and generally has less effect for backscattering. Therefore, while curved wave formulas may be important for accurate calculation, there are larger errors elsewhere in the theory. Multiple scattering must be part of the answer: as figure 4 illustrates, forward scattering is large in the ARPEFS energy range and should not be neglected. Our results here predict that this forward scattering cannot be calculated within the plane wave formulation. There may also be errors in the inelastic scattering and thermal averaging. We must investigate these questions in further work. 
APPENDIX A: ALTERNATE DERIVATION FOR DIFFERENTIAL FORM OF ORIGIN-SHIFT ADDITION THEOREM

We may arrive at the results of section II by an entirely different route. We will use a series of well known formulas conventently tabulated in Pendry, 1 . his appendi $x$ A.

We begin with the origin-shift addition theorem for $(1, m)=(1,0)$, equation 12. Using the definition of $Y_{10}$ and the recursion relation for $Y_{\ell m}$ we $f$ ind

$G_{10, \ell^{\prime \prime} m^{n}}=4 \pi \sum_{\ell^{\prime} m^{\prime}} i^{\ell^{\prime}} h_{\ell^{\prime}}(k a) Y_{\ell^{\prime} m^{\prime}}^{*}(a)\left\{\left(\frac{3}{4 \pi}\right)^{1 / 2} A_{\ell^{n}+1,-m^{n}}^{0}\right.$

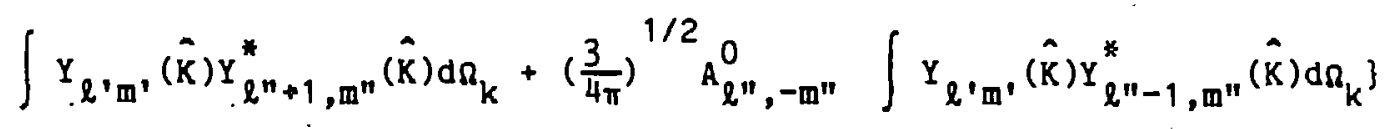

In other words, since $Y_{.10}$ is proportional to $\cos (\theta)$, the product $Y_{10} Y^{*} \ell^{\prime \prime}$ $m^{\prime \prime}$ becomes the recursion relation for $Y_{\ell^{n} m^{n}}$. The factor

$A_{\ell m}^{0}=\left[\frac{(\ell+m)(\ell-m)}{(2 \ell+1)(2 \ell-1)}\right]^{1 / 2}$

is related to the ratios of the normalizing coefficients of spherical harmonics. The remaining integrals in $G_{10, \ell " m " ~}$ are the orthonormality conditions for spherical harmonics;

$\int Y_{\ell^{\prime} m^{\prime}}(\hat{K}) Y_{\ell^{n}+1, m^{n}}(\hat{K}) d \Omega_{K}=\delta_{\ell^{\prime}, \ell^{n}+1^{\prime} \delta^{\prime} m^{n}}$ 


$$
\begin{aligned}
& G_{10, \ell " m^{n}}=\left(\frac{3}{4 \pi}\right)^{1 / 2} 4 \pi\left\{A_{\ell+1,-m^{m}}^{0} 1^{\ell "+1} h_{\ell "+1}(k a) Y_{\ell n+1, m^{\prime \prime}}^{*}(\hat{a})\right. \\
& \left.+A_{\ell ",-m^{\prime \prime}}^{0} 1^{\ell "-1} h_{\ell n-1}(k a) Y_{\ell n-1, m^{n}}^{*}(\hat{a})\right\}
\end{aligned}
$$

The factor inside the braces is the result of a differential operation on $1^{l^{n}} h_{\ell^{n}}(k a) Y_{l^{n}, m^{n}}^{*}$. Rewriting Nozawa's ${ }^{12}$ equation 3.8 in terms of normalized spherical harmonics shows that

$$
\begin{aligned}
& \left(-1 \frac{\partial}{k \partial z}\right) 1^{\ell^{n}} h_{\ell^{\prime \prime}}(k a) Y_{\ell^{\prime \prime} m^{\prime \prime}}^{*}(\hat{a})=\left\{A_{\ell^{n}+1,-m^{\prime \prime}}^{0}{ }^{\ell+1} h_{\ell "+1}(k a) Y_{\ell^{n+1, m "}}^{*}(\hat{a})\right. \\
& \left.+A_{\ell n,-m^{n}}^{0} 1^{\ell^{n}-1} h_{\ell^{n-1}}(k a) Y_{\ell^{n}-1, m^{n}}^{*}(\hat{a})\right\}
\end{aligned}
$$

Thus

$G_{10 \ell m^{n}}=\left(\frac{3}{4 \pi}\right)^{1 / 2} 4 \pi\left(-1 \frac{\partial}{k \partial z}\right) 1^{\ell n} h_{\ell n}(k a) Y_{l m^{n n}}^{*}(\hat{a})$

with

$$
\begin{aligned}
\left(\frac{\partial}{k \partial z}\right) & =\cos \theta_{\varepsilon a} \frac{\partial}{k(\partial a)}-\frac{\sin \theta_{\varepsilon a}}{k a} \frac{\partial}{\partial \theta_{\varepsilon a}} \\
& =\cos \theta_{\varepsilon a} \frac{\partial}{k(\partial a)}-\frac{\left(\cos \theta_{\varepsilon R}-\cos \theta_{\varepsilon a} \cos \theta_{a R}\right)}{(k a)} \frac{\partial}{\partial\left(\cos \theta_{a R}\right)}
\end{aligned}
$$

The addition theorem for spherical harmonics then leads to the results In equation (19). We can avold the derivative operation altogether by 
applying the recursion formulas for $h_{\ell^{n}}$ and $Y_{\ell^{n} m^{n}}$, but this approach is tedious. 


\section{APPENDIX B. APPLICATION TO EXAFS}

We apply the approach of section II to the spherical-wave singlescattering Extended X-ray Absorption Fine Structure. Schaich ${ }^{23}$ and Gurman, et al. 24 recently derived simplified, exact EXAFS formulas for cubic or polycrystalline environments. Here we consider only (1s) corelevel excitation and linearly polarized light, but we allow a general environment. With the $\hat{z}$ axis along the polarization vector, the dipole selection rules reduce Schaich's equation (3) for the $x$-ray absorption coefficient to

$$
\mu_{c}=A_{4} \operatorname{Im}\left[M_{01}^{2}\left(1+\chi_{1}\right)\right]
$$

Our $x_{1}$ corresponds to Schaich's $\left(1+x_{11}\right)$. We are interested only in the oscillations, $x_{1}$; we refer to schaich for the radial matrix elements $M_{01}$ and constants in $A_{4}$. Transcribing Schaich's equation (5) into our notation gives

$$
x_{1}(k)=\sum_{\vec{a} \neq 0<\ell^{\prime \prime m}}\left[e^{1 \delta_{1}^{\prime}}(1) G_{\ell " m^{\prime \prime} 10}(-k \vec{a})(-1) T_{\ell "}(k) G_{10 \ell " m^{\prime \prime}}(k \vec{a})(-1) e^{1 \delta_{1}^{\prime}}\right]
$$

The factor $e^{1 \delta^{\prime}} \ell$ is the absorber atom phase shift which cancels in the photoelectron diffraction experiment and hence was dropped from the formulas of section II.

To apply the differential form from appendix $A$, note that 
$G_{\ell " m " 10}(-k \vec{a})=(-1)^{m "} G_{10 \ell "-m "}(-k a \vec{a})$

This is a consequence of the conjugation property of spherical harmonics With the differentiai forms for the origin-shift addition theorem coefficients, we have

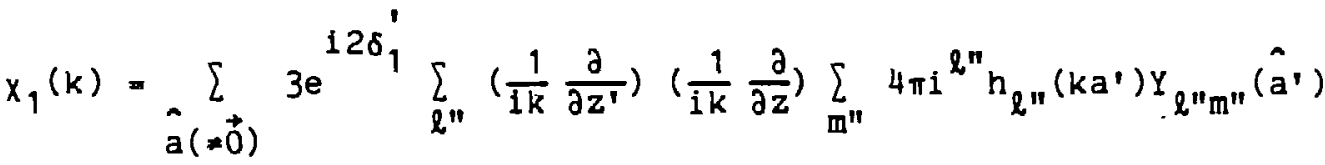

$$
\begin{aligned}
& (-1) T_{\ell "}(k) !^{\ell "} h_{\ell "}(k a) Y_{\ell " m^{n}}^{*}(\hat{a})
\end{aligned}
$$

The primes distinguish outgoing and backscattered waves until the derivatives are complete. The addition theorem for spherical harmonics simplifies this expression, and we employ our separation of spherical waves into asymptotic and polynomial parts to write

$$
\begin{aligned}
x_{1}(k)= & -\sum_{\vec{a}(\neq 0)} 3 e^{12 \delta_{1}^{\prime}} \cdot \sum_{\ell^{\prime \prime}}(2 \ell+1)\left(\frac{1}{1 k} \frac{\partial}{\partial z^{\prime}}\right) h_{0}\left(k a^{\prime}\right) d_{\ell^{\prime \prime}}\left(k a^{\prime}\right) \\
& \left(\frac{1}{1 k} \frac{\partial}{\partial z}\right) h_{0}(k a) d_{\ell}(k a) P_{\ell^{\prime \prime}}\left(\cos \theta_{a a^{\prime}}\right)
\end{aligned}
$$

where $\cos \theta_{a a}$, will ultimately be -1 . The first derivative becomes

$$
\begin{aligned}
& \left(\frac{1}{i k} \frac{\partial}{\partial z}\right) h_{0}(k a) d_{\ell "}(k a) P_{\ell "}\left(\cos \theta_{a a}\right) \\
& \quad=h_{0}(k a)\left[d_{1}(k a) d_{\ell "}(k a) \cos \theta_{\varepsilon a} P_{\ell "}\left(\cos \theta_{a a^{\prime}}\right)\right.
\end{aligned}
$$




$$
\begin{aligned}
& +\frac{\cos \theta_{\varepsilon a}}{1 k} \frac{\partial d_{\ell^{\prime \prime}}(k a)}{\partial(a)} P_{\ell^{\prime \prime}}\left(\cos \theta_{\varepsilon a^{\prime}}\right) \\
& +\frac{\left(\cos \theta_{\varepsilon a^{\prime}}-\cos \theta_{\varepsilon a} \cos \theta_{\varepsilon a^{\prime}}\right)}{1 k a} d_{\ell^{\prime \prime}}(k a) \frac{\partial P_{\ell}\left(\cos \theta_{a a^{\prime}}\right)}{\partial\left(\cos \theta_{a a^{\prime}}\right)}
\end{aligned}
$$

The first term in this expression is a consequence of the derivative as a lifting operator. After the second derivative we may set $a^{\prime}=a, P_{\ell}\left(\cos \theta_{a a^{\prime}}\right)=(-1)^{\ell}$, and $\left[\cos \theta_{\varepsilon a^{\prime}}-\cos \theta_{\varepsilon a} \cos \theta_{a a^{\prime}}\right]=0$. We also need the value of $\mathrm{dP}_{\ell}(x) / \mathrm{dx}$ for $x=(-1)$; it is equal to $(-1)^{\ell+1} \ell(\ell+1) / 2$. Thus we have

$$
\begin{aligned}
x_{1}(k)= & -\sum_{\vec{a}(\neq 0)} 3 e^{12 \delta_{1}^{\prime}} \frac{e^{12 k a}}{k a^{2}} \frac{1}{1 k} \sum_{\ell=0}^{\ell}(2 \ell+1) T_{\ell}(k)(-1)^{\ell} \\
& \left\{\cos ^{2} \theta_{\varepsilon a}\left[d_{1}(k a) d_{\ell}(k a)-1 \frac{\partial d_{\ell}(k a)}{\partial(k a)}\right]^{2}-\frac{\sin ^{2} \theta_{\varepsilon a}}{(k a)^{2}}\left[d_{\ell}(k a)\right]^{2} \frac{\ell(l+1)}{2}\right\}
\end{aligned}
$$

This form most clearly displays the origin of the curved wave corrections, but to compare to the work of Schalch, note that

$$
\frac{1}{1 k} \frac{\partial d_{\ell}(k a)}{\partial(a)}=d_{\ell-1}(k a)-d_{\ell}(k a)-\frac{\ell}{1 k a} d_{\ell}(k a)
$$

which--together with the recursion relation for $d_{\ell}(k a)--$ shows that

$$
\left[d_{1}(k a) d_{\ell}(k a)+\frac{1}{1 k} \frac{\partial d_{\ell}(k a)}{\partial(a)}\right]=\left[\frac{\ell+1}{2 \ell+1} d_{\ell+1}+\frac{\ell}{2 \ell+1} d_{\ell-1}\right]
$$


The square of this factor may be reduced with the help of the square of the recursion relation for $d_{\ell}$ to $g i$ ve

$\left[\frac{\ell+1}{2 \ell+1} d_{\ell+1}+\frac{\ell}{2 \ell+1} d_{\ell-1}\right]^{2}=\left[\frac{\ell+1}{2 \ell+1} d_{\ell+1}^{2}+\frac{\ell}{2 \ell+1} d_{\ell-1}^{2}+\frac{\ell(\ell+1)}{(k a)^{2}} d_{\ell}^{2}\right]$

Then we can define

$f^{I S O}(\pi)=\frac{1}{I k} \sum_{\ell=0}^{\ell}(2 \ell+1) T_{\ell}(k)(-1)^{\ell}\left[\frac{\ell+1}{2 \ell+1} d_{\ell+1}^{2}+\frac{\ell}{2 \ell+1} d_{\ell-1}^{2}\right]$

and

$\mathrm{f}^{\mathrm{an}}(\pi)=\frac{1}{1 \mathrm{k}_{1}} \sum_{\ell=0}^{\ell}(2 \ell+1) \mathrm{T}_{\ell}(k)(-1)^{\ell} \frac{\ell(\ell+1)}{(k a)^{2}} \mathrm{~d}_{\ell}^{2}(k a)$

to write

$x(k)=\operatorname{Im}\left[-\sum_{a(\neq 0)} 3 \frac{e^{12 k a}}{k a^{2}} e^{12 \delta_{1}^{\prime}} \cdot\left[\left(f^{I S O}+f^{a n}\right) \cos ^{2} \theta_{\varepsilon a}-\frac{f^{a n}}{2} \sin ^{2} \theta_{\varepsilon a}\right]\right.$

In an isotropic or cubic environment, $2 \cos ^{2} \theta_{\varepsilon a}=\sin ^{2} \theta_{\varepsilon a}$ and the anisotropic scattering factor cancels out to give the same formula derived by Schaich ${ }^{23}$ and by Gurman et al ${ }^{24}$. Notice that our result demonstrates that the simplification achieved by these authors is not a consequence of symmetry--the general formula is scarcely more complicated than the high symmetry version--but rather is a result of summing over the equivalent magnetic sublevels of the scattered wave. 


\section{ACKNOWLEDGEMENTS}

This work was supported by the Director, Office of Energy Research, Office of Basic Energy Sciences, Chemical Sciences Division of the U.S. Department of Energy under Contract No. DE-AC03-76SF00098. 


\section{REFERENCES}

1. J.B. Pendry, Low Energy Electron Diffraction. Academic Press, London, (1974).

2. M.A. Van Hove and S.Y. Tong, Surface Crystallography by LEED, Springer-Verlag (1979).

3. C.B. Duke, Adv. Chem. Phys., 27, 1, (1974).

4. P.A. Lee, P.H. Citrin, P. Elsenberger, and B.M. Kincaid, Rev. Mod. Phys. 53, $769(1981)$.

5. P.A. Lee, Phys. Rev. B 13, 5261 (1976).

6. J.J. Barton, C.C. Bahr, Z. Hussaín, S.W. Robey, J.G. Tobin, L.E. Klebanoff, and D.A. Shirley, Phys. Rev. Lett. 51, 272 (1983).

7. E.L. Bullock, C.S. Fadley, P.J. Orders, Phys. Rev. B $\underline{28,} 4867$ (1983).

8. M. Abramowitz, I.A. Stegin, Handbook of Mathematical Functions, National Bureau of Standards, Appl. Math. Serv. 55, 1964.

9. L.I. Schiff, Quantum Mechanics, 3rd edition, McGraw- Hill, New York, 1968.

10. P.A. Lee and J.B. Pendry, Phys. Rev. B 11, 2795 (1975).

11. This is the same formula as given in Pendry, ref. 1, his Appendix A. Note that his factor $i^{\ell-l^{\prime}-l^{\prime \prime}}$ is equal to $1^{-l+l^{\prime}+l^{\prime \prime}}$ whenever the Gaunt integral is nonzero.

12. R. Nozawa, J. Math. Phys. I, 1841 (1966).

3. B. Van der Pol, Physica $\underline{3}, 393$ (1936).

14. When the differential operator is appiled to generate the addition theorem, derivatives may be taken with either $\vec{a}$ or $\vec{r}^{\prime}$ constant, where $\vec{a}+\vec{r} \cdot \vec{r}$. Nozawa uses the physically more appealing choice of $\vec{a}$ constant; our results follow most readily ip $\vec{r}^{\prime}$ is constant for the 
purpose of the lifting operation. The equivalence of these two avenues may be proven by, for example, considering the Fourier transform derivation of the addition theorem as given by Nozawa.

15. C. Cohen-Tannoudji, B. Diu, F. Laloe, Quantum Mechanics, John Wiley and Sons, New York, 1977.

16. B. Sinkovic, P.J. Orders, C.S. Fadley, R. Trehan, Z. Hussain, and J. Lecante, submitted to Phys. Rev. B.

17. J.J. Barton, C.C. Bahr, Z. Hussain, S.W. Robey, L.E. Klebanoff, and D.A. Shirley, Soc. Photo-Optical Instr. Eng. 447, 82 (1984); J.J. Earton and D.A. Shirley, "A Generalized Ramsauer-Townsend Resonance in ARPEFS Oscillations," LBL-19325, to be published, and Chapter 8. 18. J.J. Rehr, E.A. Stern, R.L. Martin, and E.R. Davidson, Phys. Rev. B 17, $560(1978)$.

19. P. Eisenberger and B. Lengeler, Phys. Rev. B 22, 3551 (1980).

20. P.H.Citrin, P. Eisenberger, and R.C.Hewitt, Phys. Rev. Lett. 45 , 1948, (1980).

21. H.A. Bethe and R. Jackiw, Intermediate Quantum Mechanics, 2nd edition, W.A. Benjamin, Reading, MA., 1968.

22. B.K. Teo, Proc. Intl. Conf. EXAFS and Near Edge Structure, ed. A. Bianconi, L. Incoccia, and S. Stipcich, Springer-Verlag 1983, p. 11. 23. W.L. Schalch, Phys. Rev. B 29, 6513 (1984); J.E. Müler and W.L. Schaich, Phys. Rev. B 27, 6489 (1983).

24. S.J. Gurman, N. Binsted, and I. Ross, J. Phys. C 17, 143 (1984). 


\section{FIGURE CAPTIONS}

Figure 1. Scattering factor amplitude in $\AA$ for $N i$ aton potential at $k$ $=5 \mathrm{~A}^{-1}(95 \mathrm{eV})$. Solid line is $\left|\mathrm{f}_{\mathrm{aR}}^{00}\right|$, the $\ell=0$ spherical wave scattering factor; dashed line, plane wave limit 2ka \> $\ell(\ell+1)$. Right hand panel gives cartesian plots of factor magnitude versus scattering angle, $\theta_{\mathrm{aR}}$ in degrees; leit hand panel is a polar plot with $\theta_{a R}=0^{\circ}$ running up the figure.

Figure 2. Radial derivative scattering factor amplitude, $\left|\mathrm{f}_{\mathrm{aR}}^{10}\right|$ in A for $\mathrm{Ni}$ aton potential at $\mathrm{K}=5 \mathrm{~A}^{-1}(95 \mathrm{eV})$. Format described in Figure 1. Note the scale of this figure is $1 / 20$ th of Figure 1 .

Figure 3. Angular deri iative scattering factor amplitude $\left|f_{a R}^{01}\right|$ times sine of the scattering angle, $\theta_{a R}$, in $\&$ for $\mathrm{Ni}$ potential at $k=5 \AA^{-1}(95 \mathrm{eV})$. Format described in Figure 1. This scattering factor cannot contribute in near forward or near backscattering directions.

Figure 4. Identical to Figure 1, except $k=10 \mathrm{~A}^{-1}(381 \mathrm{eV})$. Note the Improved accuracy of the plane wave limit for backscattering angles. The figure has the same scale as figure 1 to emphasize backscattering angles.

Figure 5. Identical to Figure 2 except for $k=10 \mathrm{~A}^{-1}(381 \mathrm{eV})$. Figure 6. Identical to Figure 3, except for $k=10 \AA^{-1}(381 \mathrm{eV})$. Note that shift of the main peak to lower angles; its amplitude is similar to the amplitude of the main peak at $k=5 \AA^{-1}$, but the correction for backscattering is very much smaller now. 
Figure ?. Difference in phase (in radians) between spherical and plane-wave models versus scattering angle $\theta_{\mathrm{aR}}$ in degrees, for scattering frum $N i$ atom potentials at $k=5 \mathrm{~A}^{-1}$.

Figure 8. Scattering factor amplitudes in $\$$ versus electron wavenumber $\left(A^{-1}\right)$ for near backscattering $\left(\theta_{a R}=173^{\circ}\right)$ from $N i$ atom potentials. Solid line, basic spherical scattering factor $\mathrm{P}_{\mathrm{aR}}^{00}$; circles, plane-wave limit scattering factor $\mathrm{f}_{\mathrm{aR}}$; triangles, radial derivative scattering factor $\mathrm{f}_{\mathrm{aR}}^{10}$. The plane-wave factor is rather close to the spherical wave $f_{a R}^{00}$ and the other spherical wave corrections are very small at all energies; notice also that plane wave error approaches a non-zero constant.

Figure 9. Scattering factor amplitude in $\AA$ versus electron wavenumber $\left(A^{-1}\right.$ ) for forward scattering $\left(\theta_{a R}=0^{\circ}\right)$. Solid line, basic spherical scattering factor $\rho_{a R}^{00}$ i circles, plane-wave limit scattering Pactor $\mathrm{P}_{\mathrm{aR}}$; triangles, radial derivative scattering factor $\mathrm{f}_{\mathrm{aR}}^{10}$. Note the nearly constant plane-wave error; the radial derivative correction becomes alwost 10 percent at low energies.

Figure 10. Scattering factor amplitudes in $A$ versus electron wavenumber $\left(A^{-1}\right.$ ) for scattering through $\theta_{a R}=127^{\circ}$, the position of a Generalized Ramsauer Townsend resonance. Solid line, basic spherical wave factor $\mathrm{e}_{\mathrm{aR}}^{00}$; circles, plane-wave limit $\mathrm{f}^{\mathrm{plane}}$; crosses, angular derivative $\mathrm{f}_{\mathrm{aR}}^{01}$. The radial derivative is negligible at this angle for all energies.

Flgure 11. Phase shifts for scattering from $N i$. The dashed line shows the phase shift function $\phi_{j}$ calculated with plane-wave 
theory for $\theta=127^{\circ}$. The dotted line is the phase shift from the experimental curve. The zero crossing Jump in phase occurs too high in wavenumber in the plane-wave limit. Solid lines are curved-wave calculations of the phaise shift function for the indicated scattering angles.

Figure 12. Radius for acceptable results from plane wave cal culations versus bond angle for $k=5 \AA^{-1}$ (solid line) and $k=10 \AA^{-1}$ (line with circles). The radil were selected so that all distances greater than the plotted lines have $\left|f_{a R}^{00}-f_{a R}^{p l a n e}\right|<$ 0.06 .

Figure 13. Scattering factors for (1s) EXAFS. Solid line is $f^{\text {iso }}$, the curved wave scattering amplitude defined by eqn. B13. Line with circles is the plane wave limit which has a similar shape to $\mathrm{f}^{\text {tso }}$. Line with triangles is $\mathrm{f}^{\text {an }}$, eqn. (B13) multiplied by a factor of 50 .

Figure 14. Schematic semiclassical orbits for an attractive putential. If the circle represents the effective radius of a screened nuclear charge, then particles with iarge impact parameters will sample only the weak outer region of the potential and scatter through small (forward) angles. Particles with small impact parameters or bit the strong nuclear at traction and exit at large (backscattering) angles. The connection to wave scattering is made through $b=l / k$ where $b$ is the impact parameter: large $\&$ partial waves contribute to forward scattering and small \& waves dominate for backscattering. 


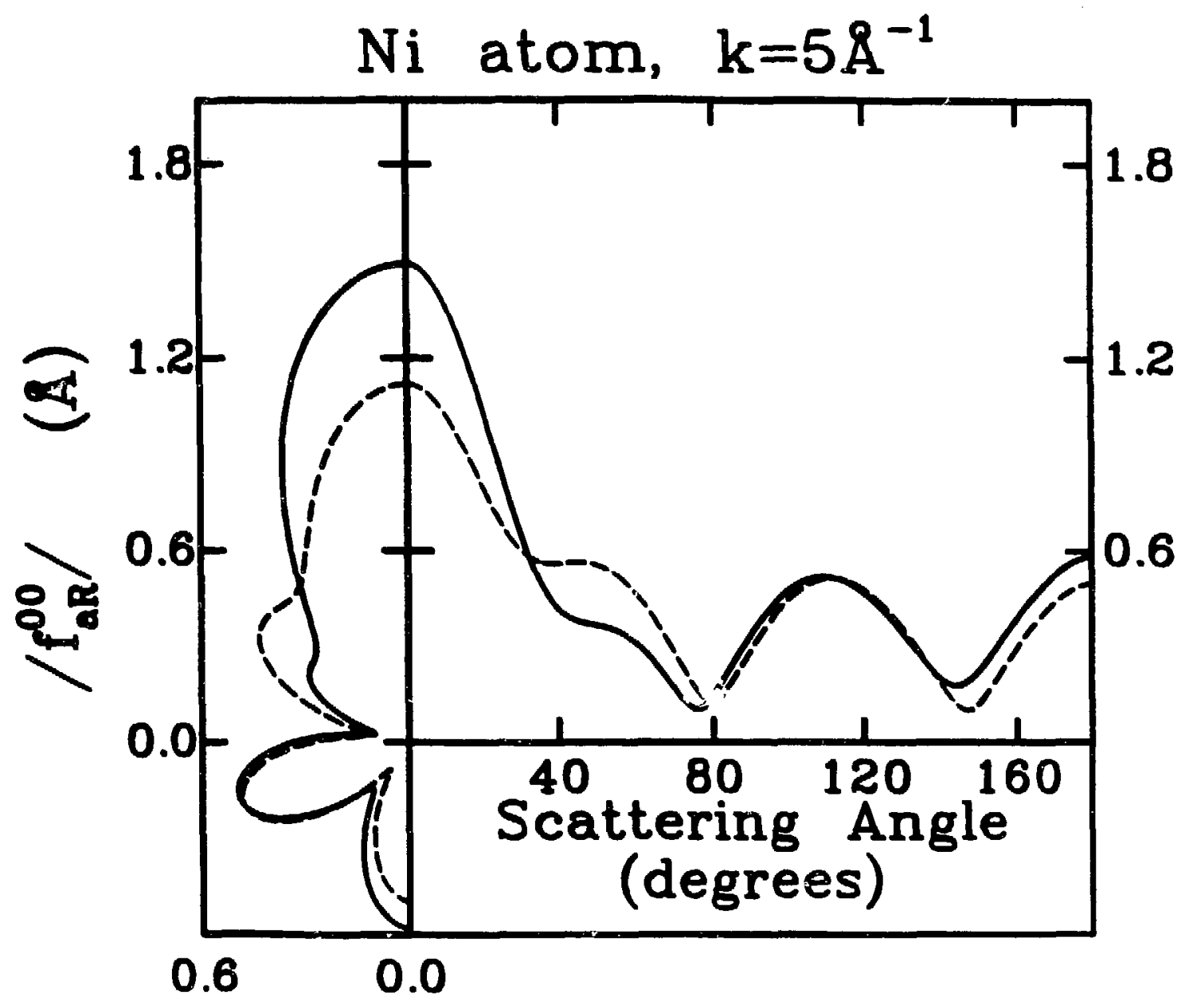

XBL 852-1310

Figure 1 


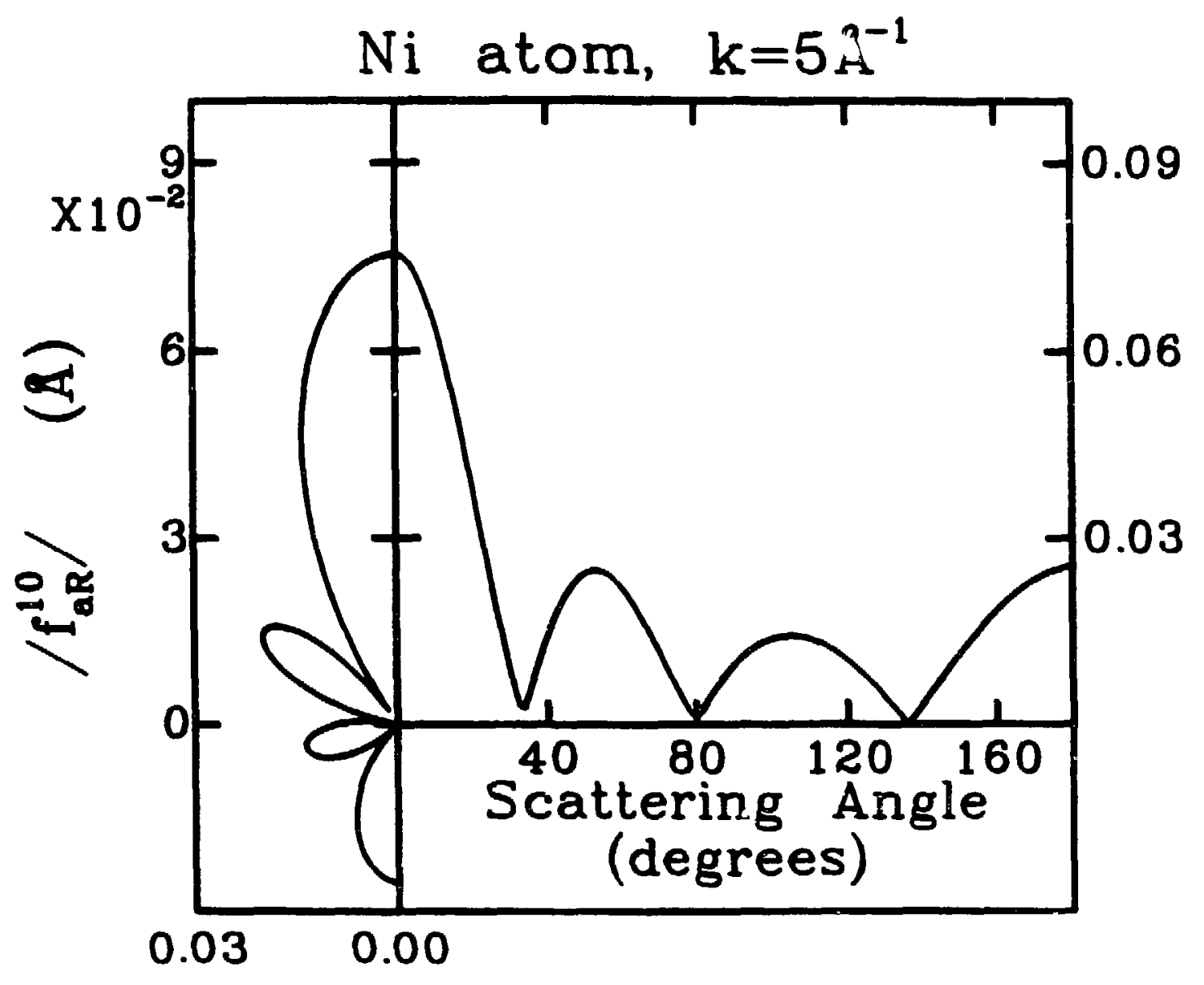

XBL 852-1311

Eigure 2 


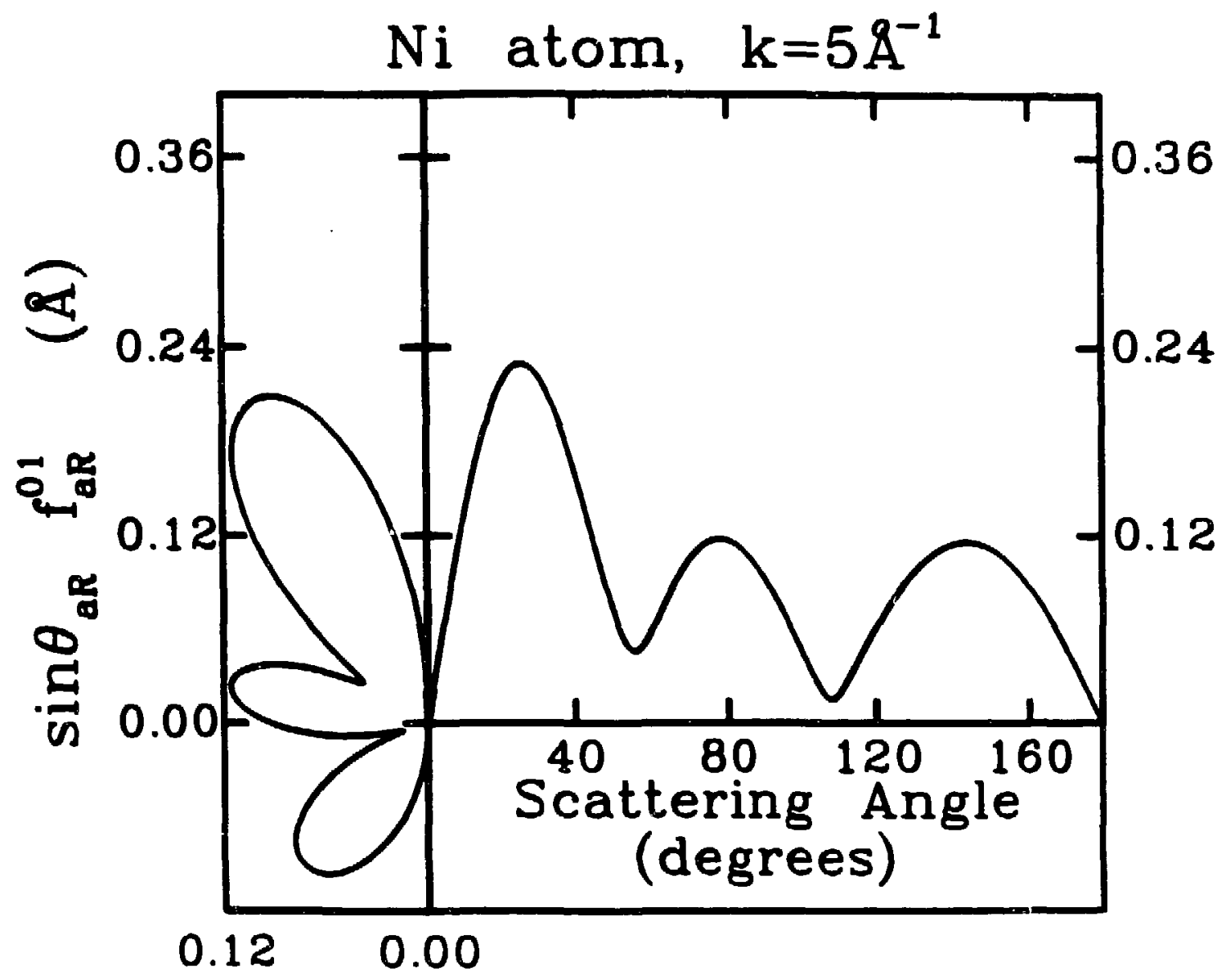

XBL 852-1312

Figure 3 


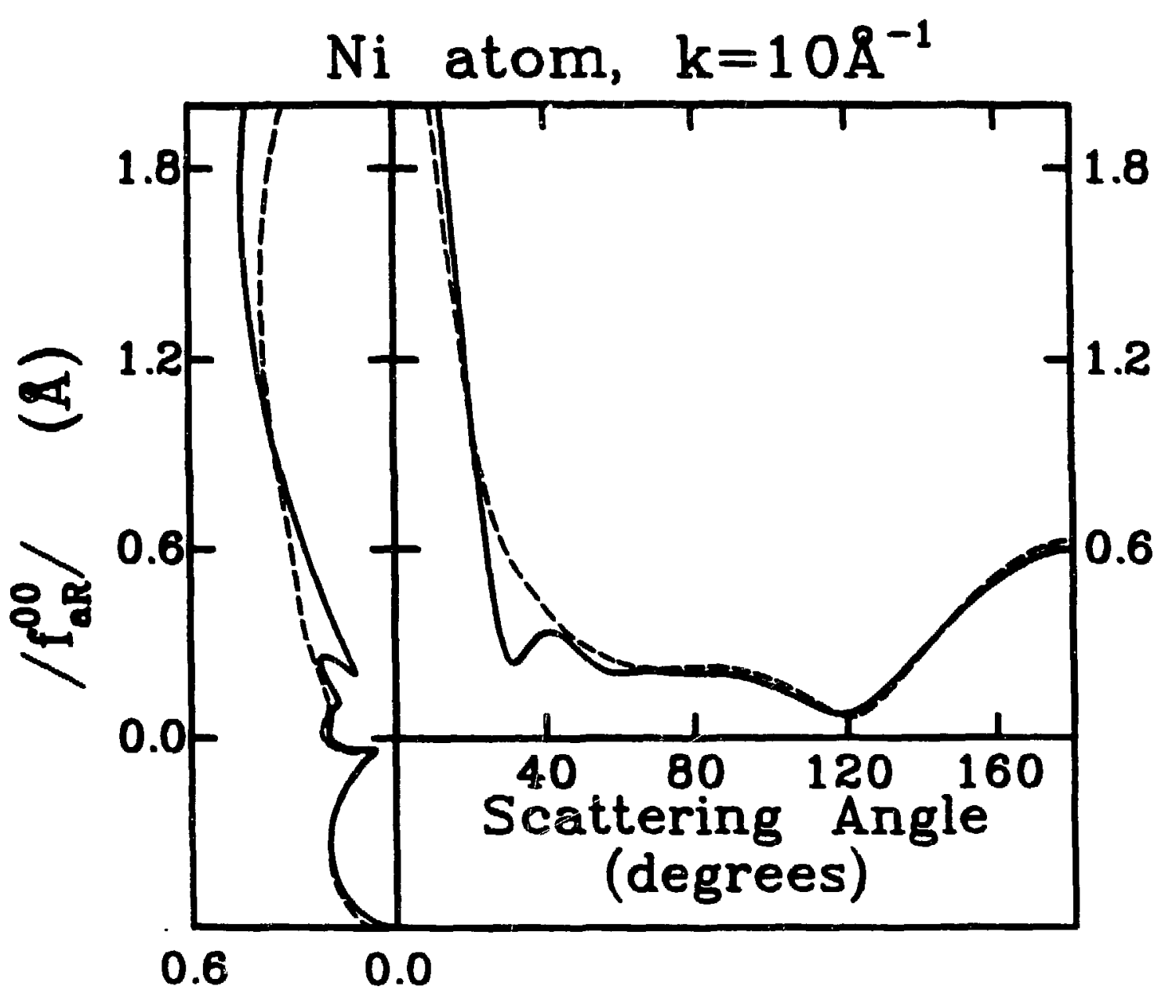

XBL 852-1313

Figure 4 


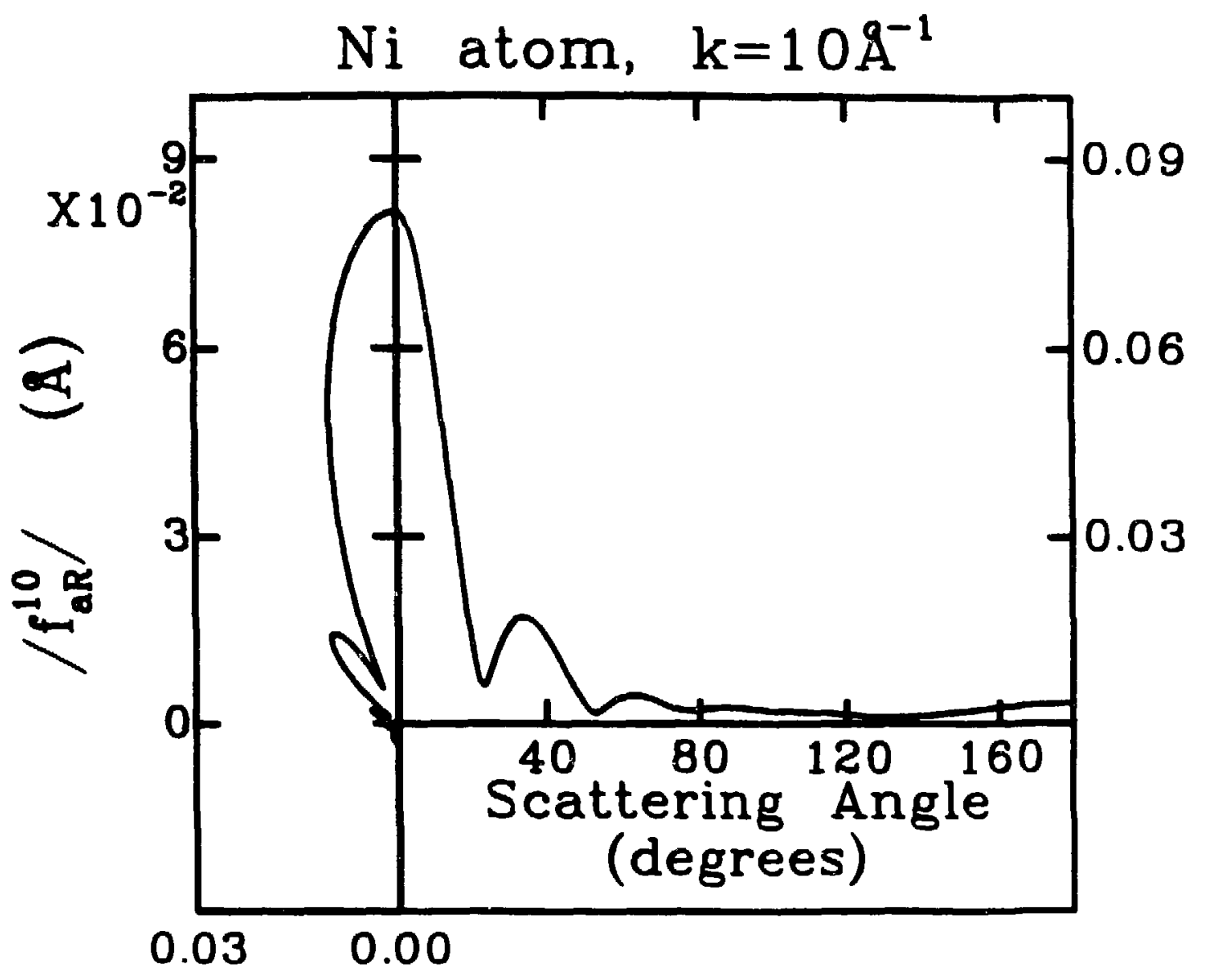

XBL 85?-1314

Figure 5 


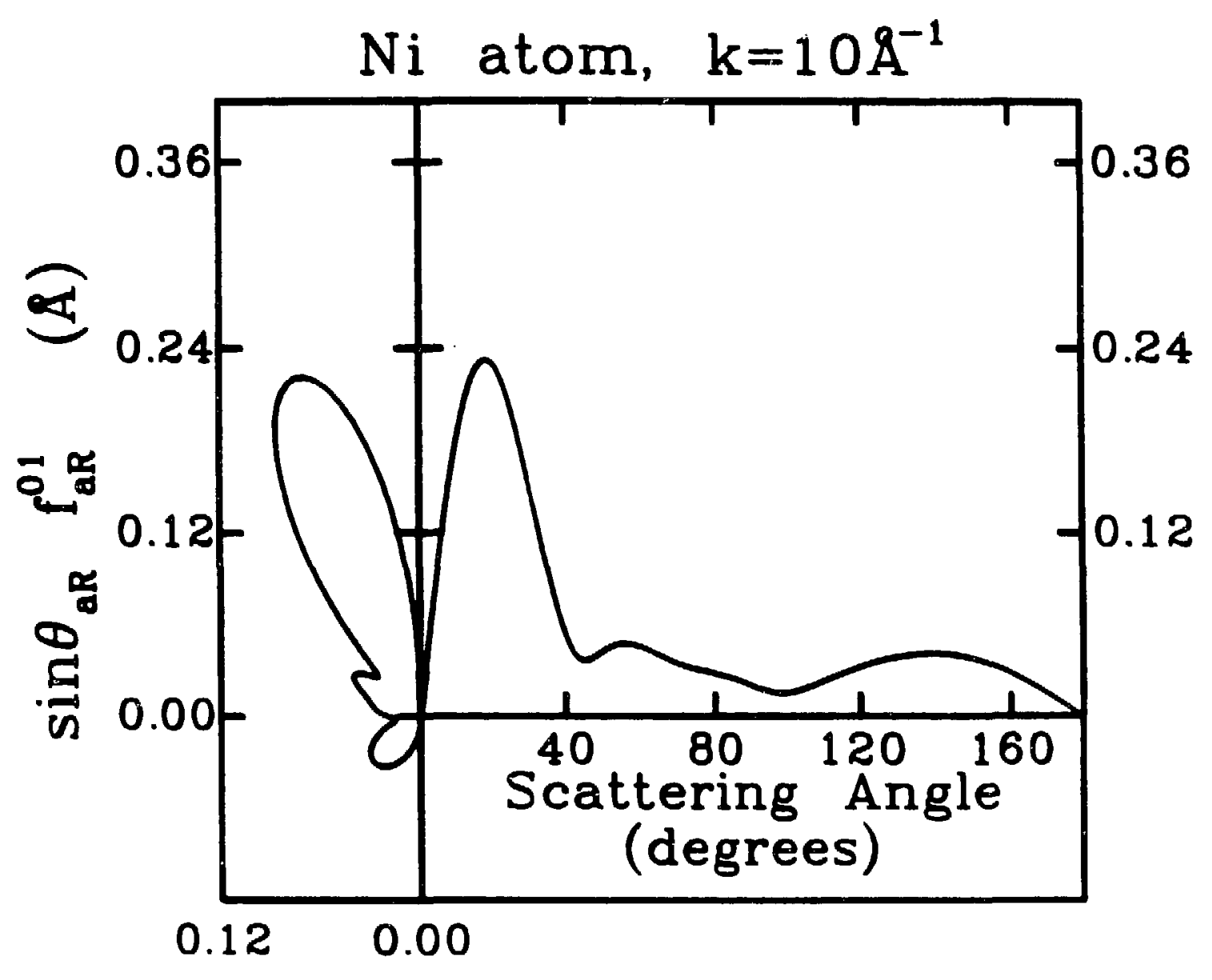

XBL 852-1315

Figure 6 
$\mathrm{Ni}$ atom

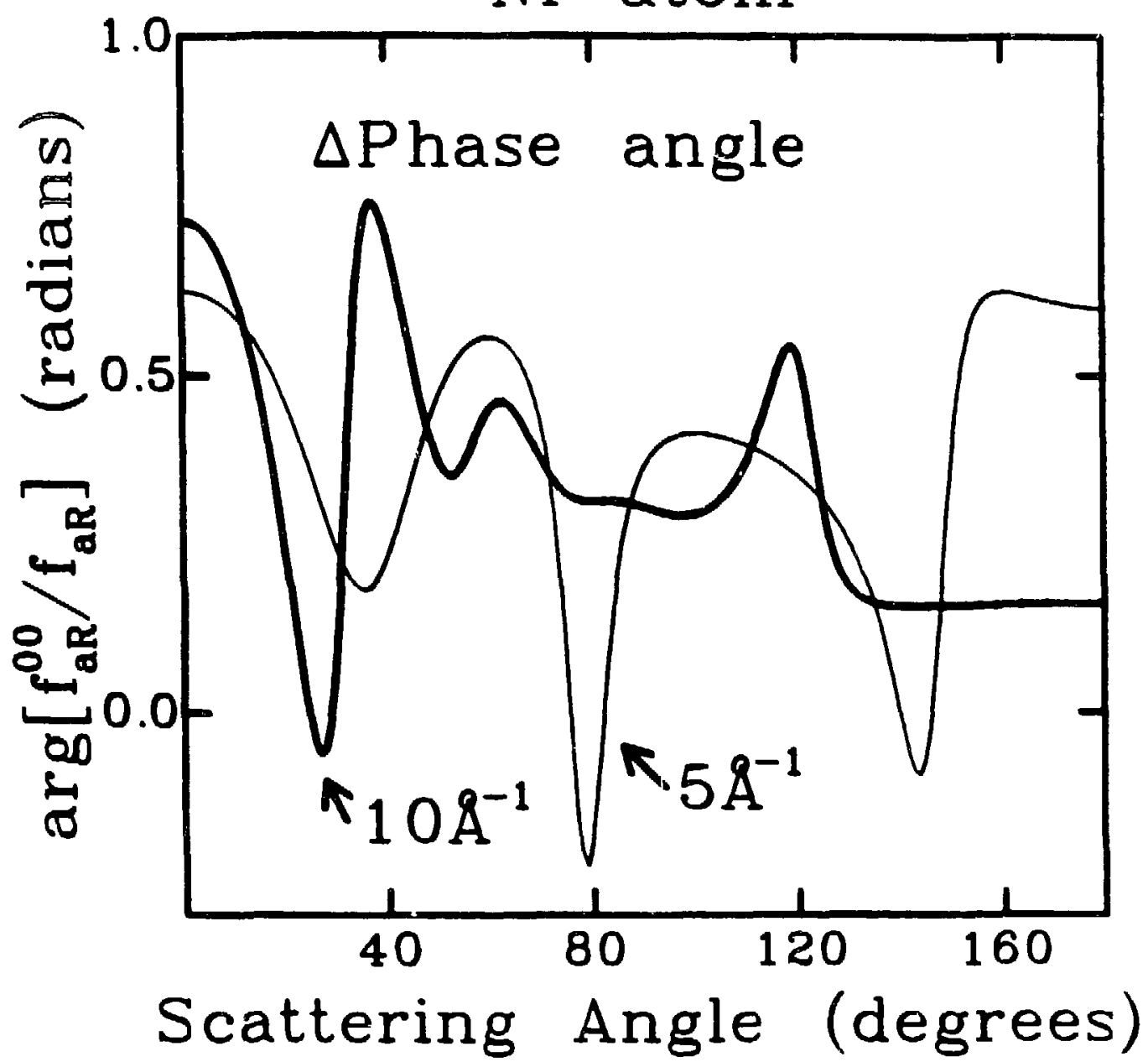

XBL $852-1316$

Figure 7 


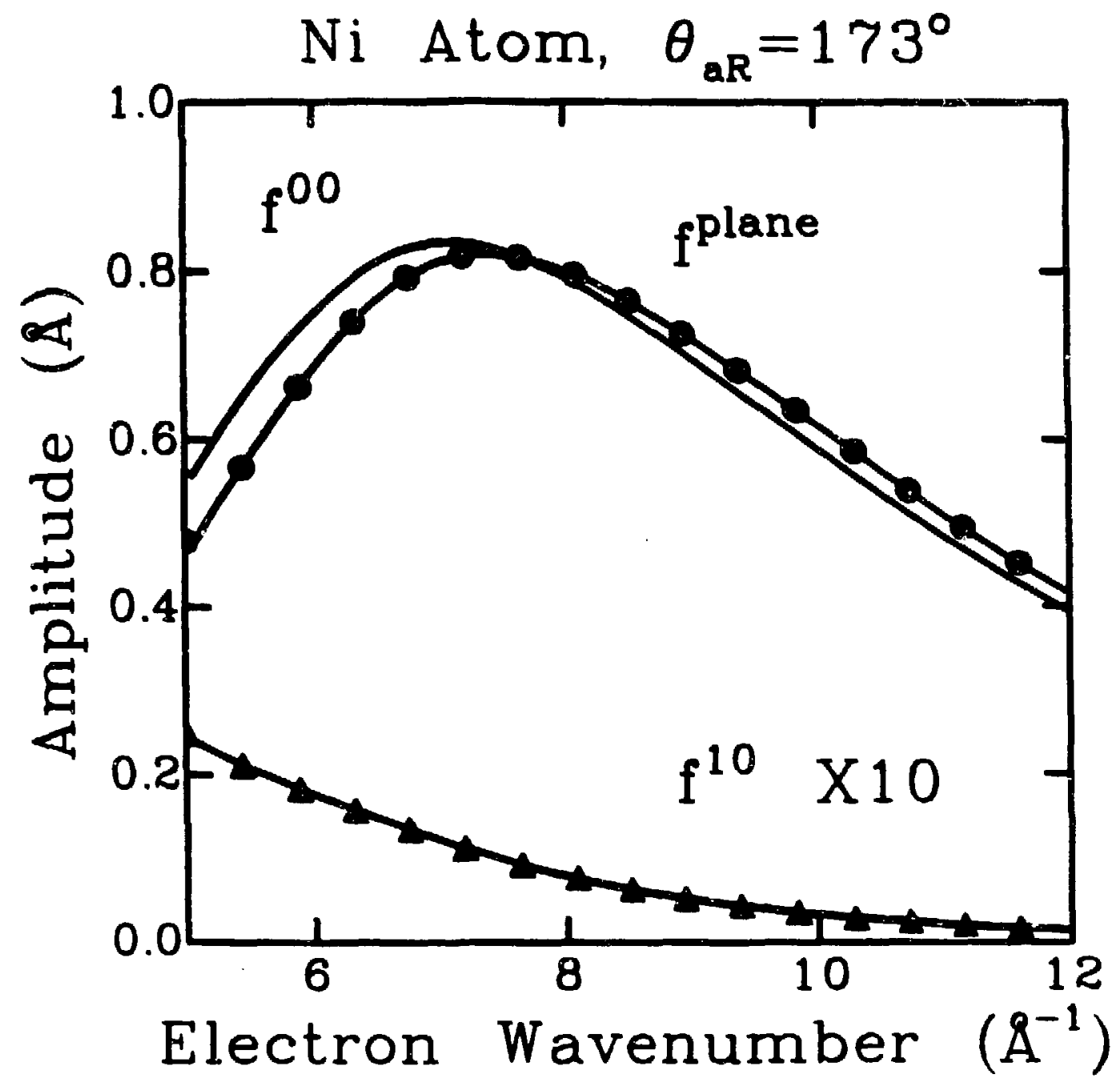

XBL 852-1318

Figure 8 


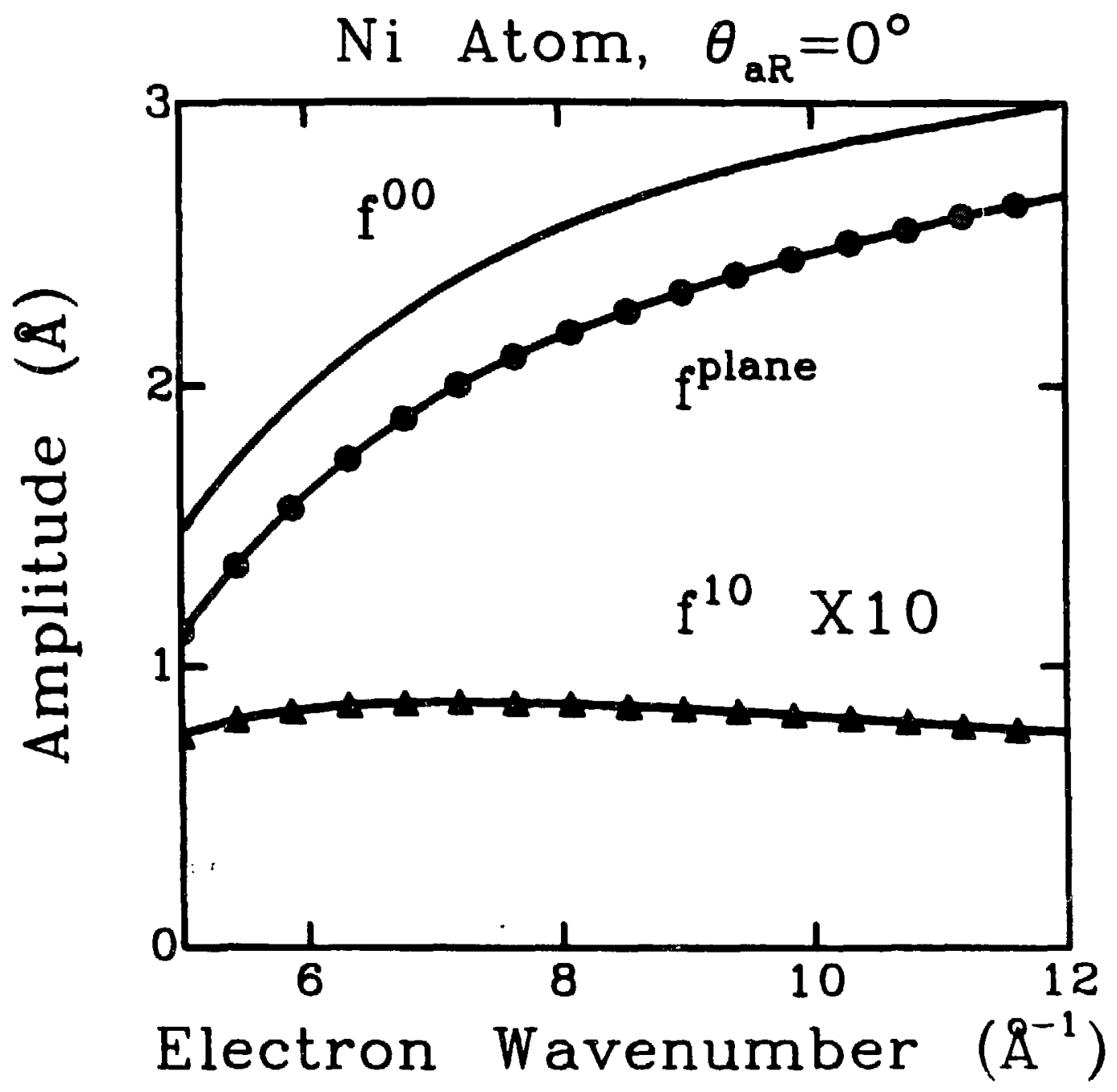

XBL 852-1317

Figure 9 


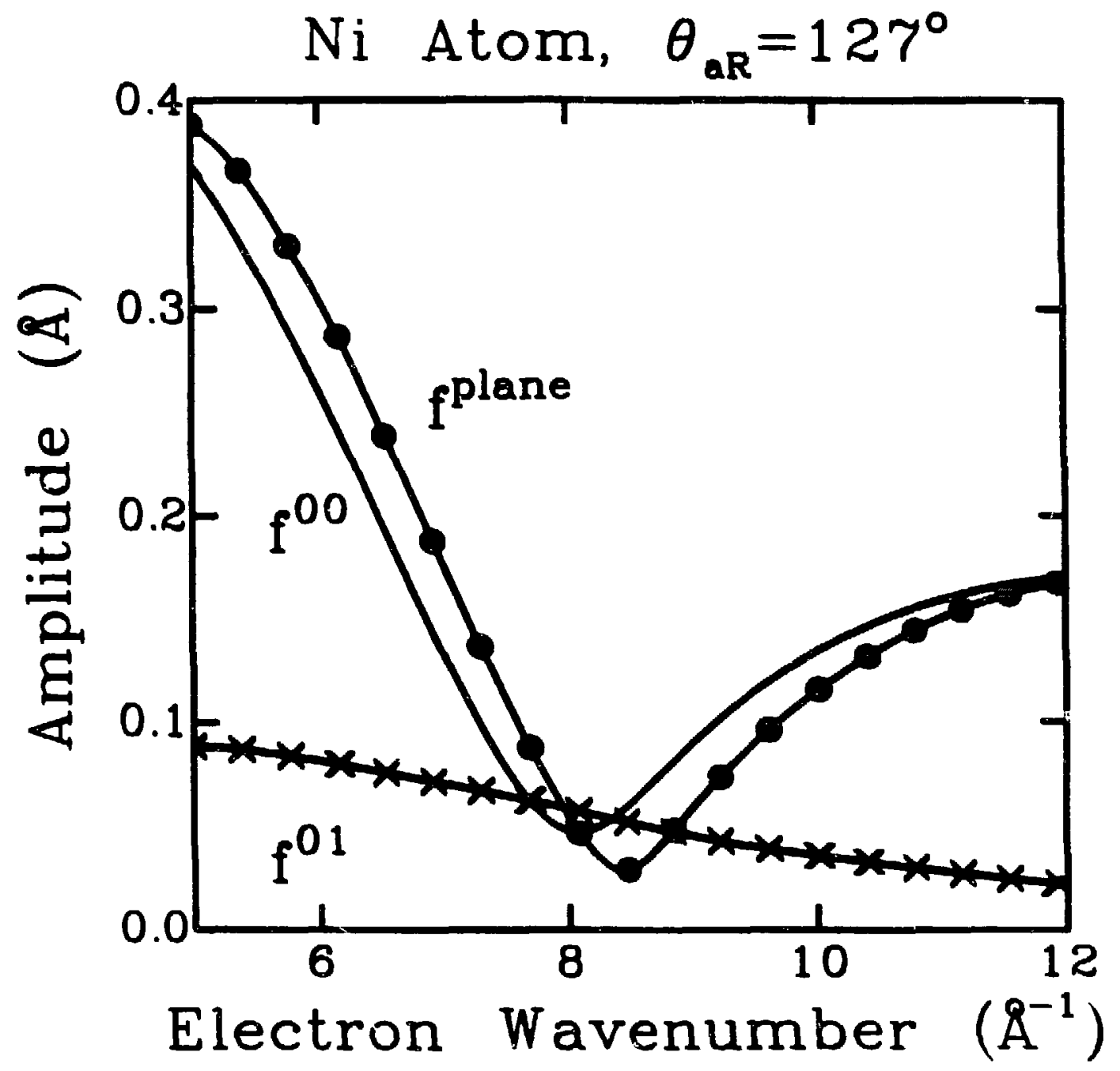

XBL 852-1319

Figure 10 


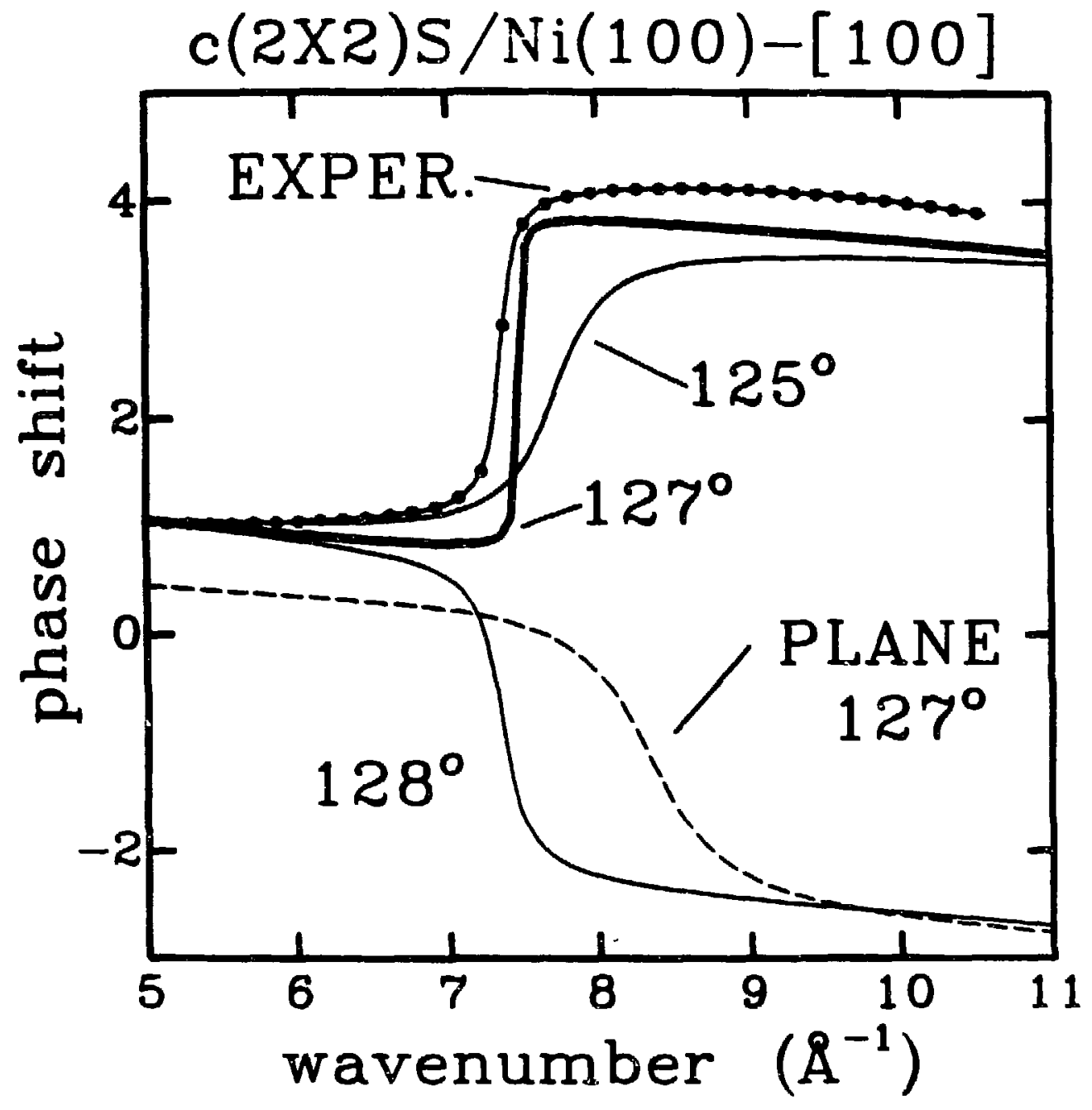

DK:NIS1 11 .DUS

XBL $848-3548$

Figure 11 
$\mathrm{Ni}$ atom

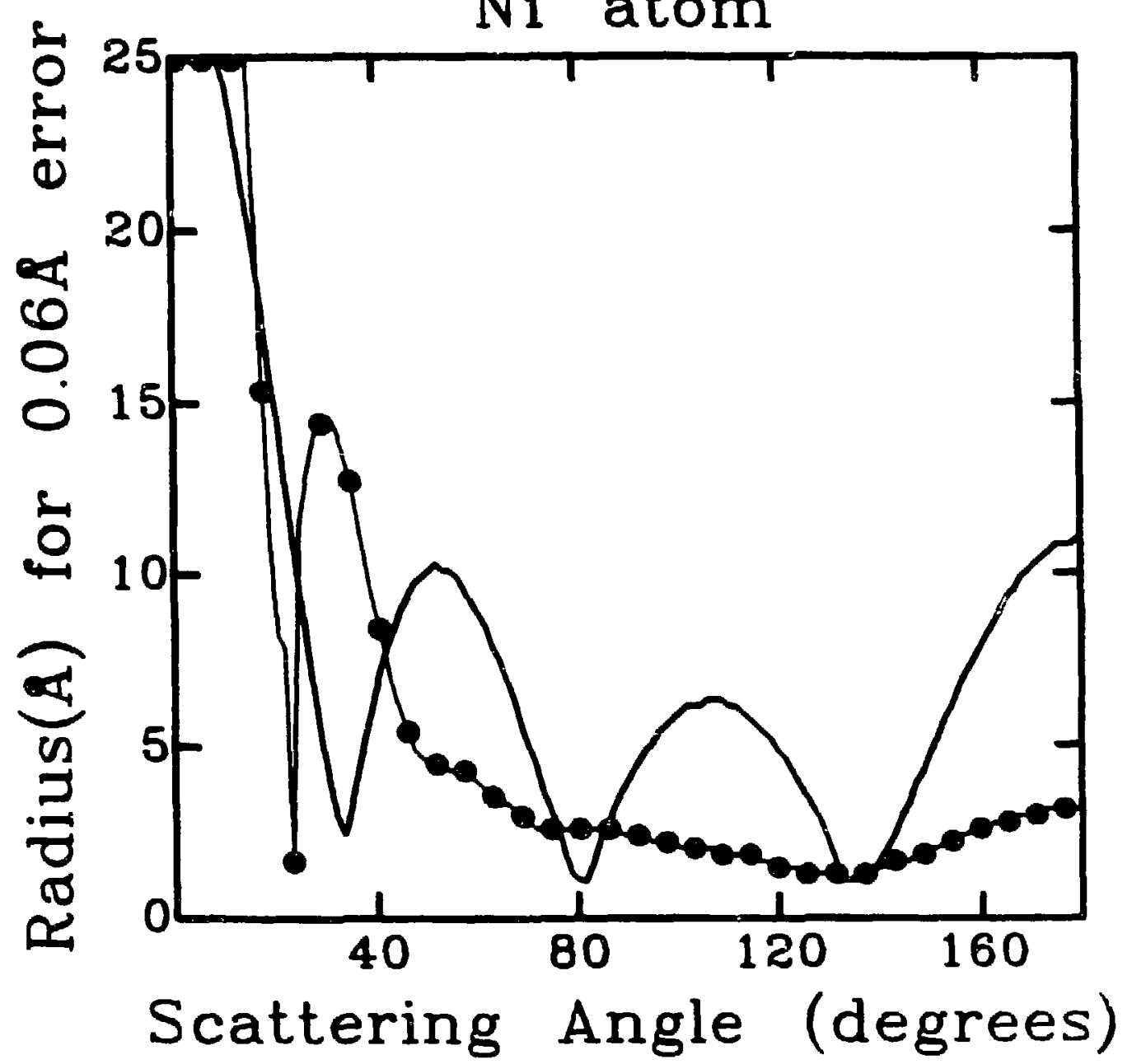

XBL 852-1320

Figure 12 


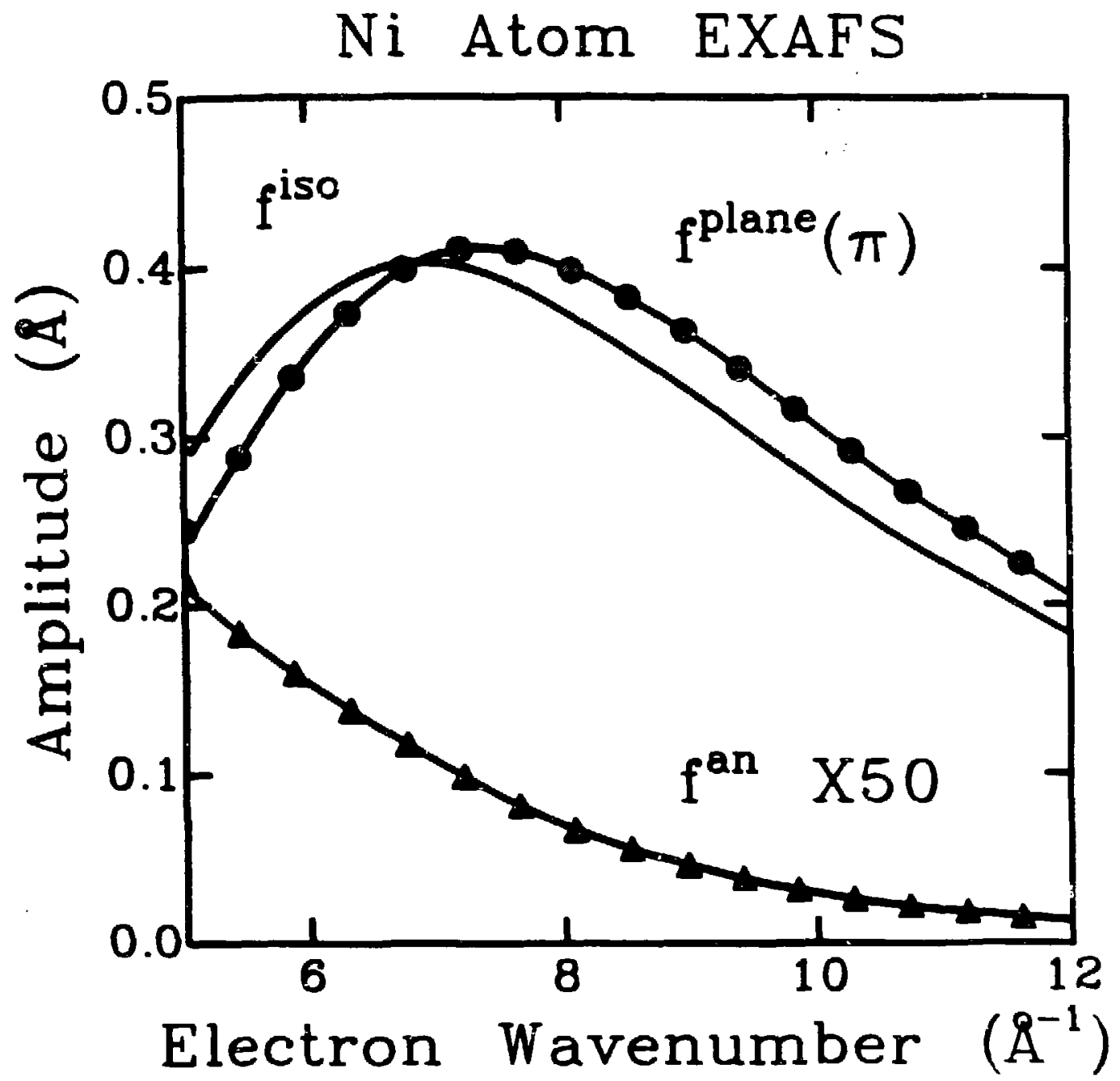

XBL 852-1321

Figure 13 


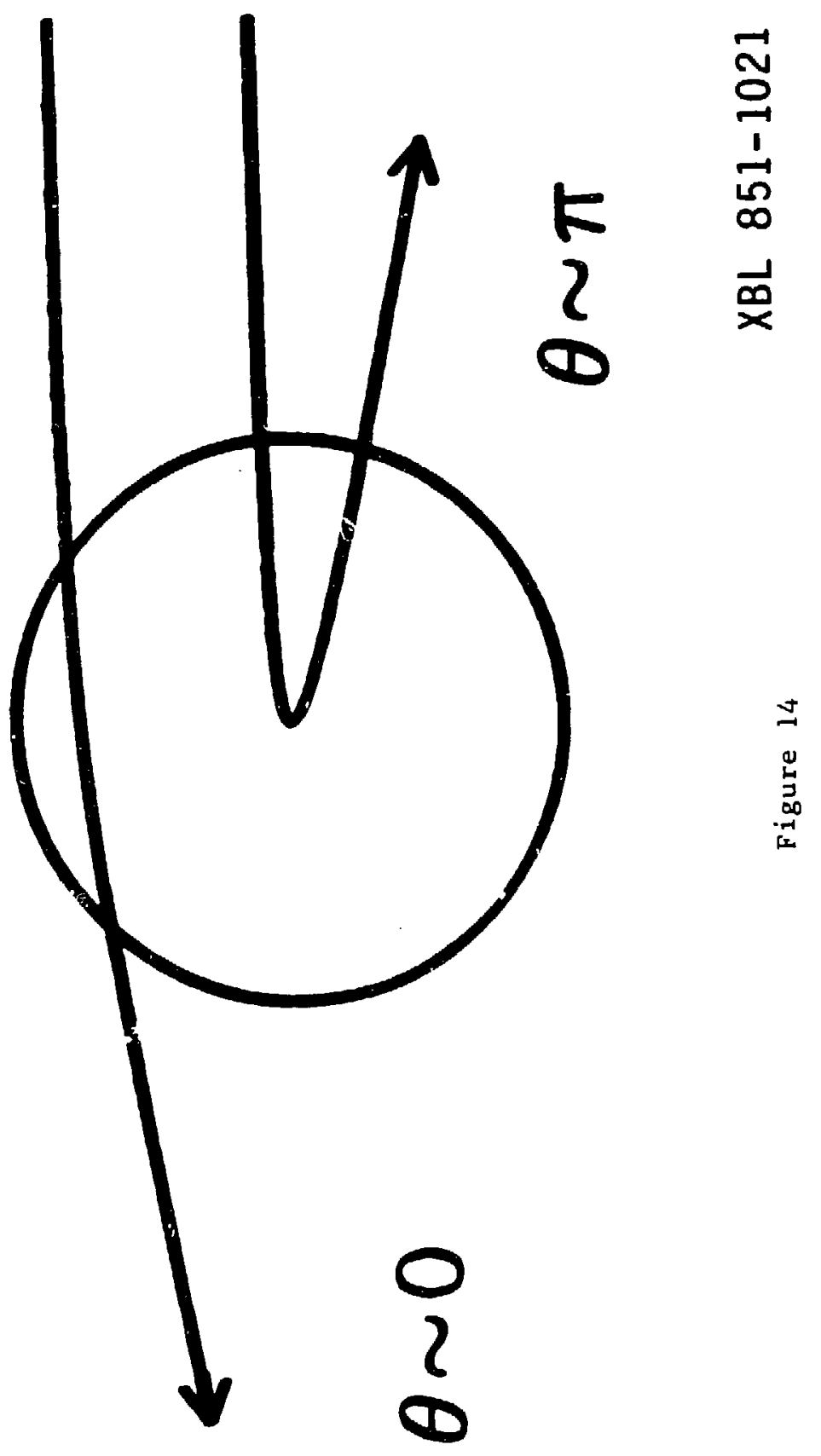


CHAPTER 3:

\section{APPROXIMATE TRANSLATION OF SCREENED SPHERICAL WAVES}

\section{ABSTRACT}

We develop and discuss a Taylor series expansion of the addition theorem for screened spherical waves, $i^{\ell_{h}} h_{\ell}(k r) Y_{\ell m}(\hat{r})$. The expansion is designed for orderly progression in the addition of curved-wavefront corrections to multiple scattering of electrons in solids, but it apfiles to any wave scattering problem based on the addition theorem for spherical waves. We relate one of the expansion indices to a magnetic quantum number in a coordinate system rotated to align the quantization and transiation axes. 
In the course of constructing an accurate model for photoelectron scattering from ion cores in a solid surface we have been struck by the wide gap between the simple and "exact" models for spherical wave scattering. The simple "plane-wave" model for electron scattering in an ensemble of localized, non-overlapping potentials treats each scattering event as if the potentials were infinitely separated. This model seems to be remarkably successful ${ }^{1}$ in the intermediate energy range (100$600 \mathrm{eV})$. The exact model incorporates the wavefront curvature effects important when the potentials are separated by distances comparable to their diameter. Although these curvature effects are considered to be important for low energies $(<100 \mathrm{eV})$, very few studies of these effects in the intermediate energy range have been undertaken because the calculations are prohibitively expensive. We began to explore the possibility that a modest improvement on the plane-wave model would allow curvature corrections to be examined without requiring the complete, exact calculation. The solution we arrived at is physically appealing and, although the mathenatical development is standard, we believe the resulting formula and its interpretation should be valuable for many scattering problems (for examples see ref 2). Thus we present the development of a finite series approximation to the addition theorem for screened spherical waves, $i_{\ell} h_{\ell}(k r) Y_{\ell m}(\hat{r})$.

\section{EXACT ORIGIN-SHIFT ADDITION THEOREM}

Calculating the amplitude of waves scattered from a central potential by the partial wave method requires an expansion of the incident wave in an angular momentum series about the potential's 
origin. If a plane wave is incident upon the potential, the expansion in an angular momentum series is well known:

$e^{i \vec{k} \cdot \vec{r}}=e^{i \vec{k} \cdot \vec{a}} \sum_{\ell " m "}^{\ell \max } 4 \pi i^{\ell "} j_{\ell "}(k b) Y_{\ell " m n}(\hat{b}) Y_{\ell " m "}^{*}(\hat{k})$

for a potential at $\vec{a}$ and $\vec{r}=\vec{a}+\vec{b}$. Here $j_{\ell}$ is the regular spherical Bessel function, $k$ is the wavenumber, and $Y_{\ell m}$ is the spherical harmonic given in Appendix A. The limit on the sum, $l_{\max }$, is governed by the effective range of the potential, $r_{0}$, according to ${ }^{3}$

$$
k r_{0}=\left[l_{\max }\left(l_{\max }+1\right)\right]^{1 / 2}
$$

If a spherical wave is incident on the potential, the expansion in spherical harmonics has been derived by Nozawa. ${ }^{4}$ If the spherical wave emanates from the origin, we may expand it at $\vec{a}$ as

$$
\begin{aligned}
& i^{\ell} h_{\ell}(k r) Y_{\ell m}(\hat{r})=\sum_{\ell " m "} G_{\ell m \ell " m "} i^{\ell "} j_{\ell "}(k b) Y_{\ell " m "}(\hat{b}) \\
& G_{\ell m \ell " m "}=\sum_{\ell^{\prime} m^{\prime}} 4 \pi i^{\ell^{\prime}} h_{\ell^{\prime}}(k a) Y_{\ell^{\prime} m^{\prime}}^{*}(\hat{a}) \int Y_{\ell m}(\hat{k}) Y_{\ell{ }^{\prime} m},(\hat{k}) Y_{\ell^{\prime \prime} m^{\prime}}^{*}(\hat{k}) d \hat{k}
\end{aligned}
$$

where $\vec{r}=\vec{a}+\vec{b}$. In the mathematical literature, this type of formula is called an addition theorem; we will refer to this equation as Nozawa's origin-shift addition theorem. Nozawa calls the screened spherical waves "Helmholtz Solid Harmonics", but we will simply call them spherical waves and further omit the superscript designating the kind of spherical Hankel function by using $h_{\ell}(k r)=h_{\ell}^{(1)}(k r)$ and $h_{\ell}^{*}(k r)=h_{\ell}^{(2)}(k r)$. The history of the formula deserves some comment. This expansion formula is widely used but only rarely is the original literature cited. We have followed Slater ${ }^{5}$ and based our work in section II on Nozawa's 
development ${ }^{4}$. We were at first unaware of the independent work by Danos and Maximon ${ }^{2}$; these authors give a historical survey of the mathematical literature on what we call the origin-shift addition theorem this ough 1965. Interestingly, these authors state that they too were unaware of some previous work on this formula. It seems that the use of the formula in solid state physics, our particular concern, may be traced to Kohn and Rostoker and to Korringa ${ }^{6}$. We use section IV to connect our work with the outlook presented by Danos and Maximon.

To understand and use this formula we must confront the integral of three spherical harmonics, called the Gaunt integral. ${ }^{7}$ This integral can be relaced to the $3 j$ vector addition (Clebsch-Gordan) symbols and Gaunt has derived an analytic formula to calculate its value. The integral is non-zero only when

$m^{\prime \prime}=m+m^{\prime}, \quad\left|\ell^{\prime \prime}-\ell\right| \leq \ell \ell^{\prime} \leq \ell^{\prime \prime}+\ell, \quad \ell^{\prime}+\ell "+\ell=$ even

Pendry ${ }^{8}$ gives a computer program tu implement Gaunt's formula. Typically a table of these Gaunt integrals are consulted in actual calculations.

The complexity of the formula for spherical wave scattering is self-evident. In computer calculations we must recognize that the number of transformation coefficients $G_{\ell m \ell "} m^{\prime \prime}$ is proportional to $\left(\ell_{\max }+1\right)^{4}$ and each coefficient requires the summation of $-\ell_{\max }$ complex numbers times the Gaunt integral. Since $l_{\max }$ is roughly proportional to $k$, scattering calculations already difficult at $k=3 A^{-1}$ become prohibitively expensive at $k=12 A^{-1}$. Even this presumes that the Gaunt integrals are calculated once and stored; for calculations to $600 \mathrm{eV}\left(l_{\max }=19\right)$ of 
order $10^{6}$ integrals are required. For these reasons we must approximate the scattering calculations.

\section{TAYLOR SERIES EXPANSION}

Now we turn to a series expansion of the origin-shift addition theorem for spherical waves. Our approach is to examine the derivation of the origin-shift addition theorem based on the Fourier transform and, by interpreting the formula physically, deduce an appropriate approximate form.

We define the Fourier transform of a spherical wave, $i^{\ell} h_{\ell}(k r) Y_{\ell m}(r)$, as:

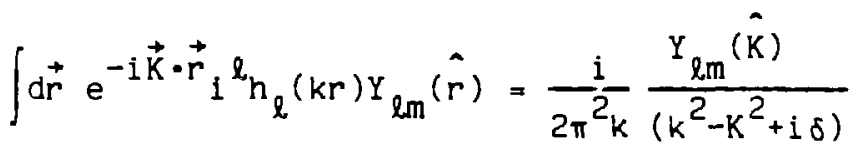

as given by Pendry. The inverse transform:

$i_{h_{\ell m}}(k r) Y_{\ell m}(\hat{r})=\frac{i}{2 \pi^{2} k} \int d \vec{K} \frac{e^{i \vec{K} \cdot \vec{r}_{Y}}(\hat{K})}{k^{2}-K^{2}+i \delta}$

leads to the addition theorem ${ }^{4}$ when we set $\vec{r}=\vec{a}+\vec{b}$. The vector $\vec{a}$ may be taken as the new origin and $\vec{b}$ as the new propagation direction for waves from $\vec{a}$. To separate radial $K$ integration from the angular integrations, the series expansion for $e^{1 \vec{K} \cdot \vec{r}}$, eqn. (1) 1s used for $e^{i \vec{k} \cdot \vec{b}}$ and $\mathrm{e}^{\mathrm{i} \overrightarrow{\mathrm{K}} \cdot \overrightarrow{\mathrm{a}}}$ : 


$$
\begin{aligned}
& i^{\ell_{h}} h_{\ell}(k r) Y_{\ell m}(\hat{r})=\sum_{\ell \prime m "} \sum_{\ell^{\prime} m^{\prime}} 4 \pi\left[\frac{2 i}{k \pi} \int_{0}^{\infty} \frac{i^{\ell^{\prime \prime} j_{\ell^{\prime \prime}}(K b) i^{\ell^{\prime}} j_{\ell^{\prime}}(K a) K^{2} d K}}{k^{2}-K^{2}+1 \delta}\right] \\
& Y_{\ell " m "}(\hat{b}) Y_{\ell^{\prime} m^{\prime}}^{*}(\hat{a}) \int Y_{\ell m}(\hat{K}) Y_{\ell^{\prime} m^{\prime}}(\hat{K}) Y_{\ell^{\prime \prime} m^{\prime \prime}}^{*}(\hat{K}) d \Omega_{K}
\end{aligned}
$$

The radial integral may be extracted from a contour integral ${ }^{9}$ to give

$$
\frac{2 i}{k \pi} \int_{0}^{\infty} \frac{i^{\ell \prime} j_{\ell^{\prime \prime}}(K b) i^{\ell \cdot} j_{\ell^{\prime}}(K a) K^{2}}{k^{2}-K^{2}+i \delta} d K=i^{\ell^{\prime \prime}} j_{\ell^{\prime \prime}}(k b) i^{\ell^{\prime}} h_{\ell^{\prime}}(k a) \quad b<a
$$

and we arrive at the addition theorem, eqn. 3. We now identify the Gaunt integral as the angular integration remaining from the inverse Fourier transform. Notice that $\mathrm{Y}_{\ell m}(\hat{K})$ is the angular part of the Fourier transform of the spherical wave.

With this derivation in mind, we return to the inverse transformation integral, eqn. (6), and claim that this is also a prescription for constructing a spherical wave by superposition of plane waves. We can then imagine constructing an approximate spherical wave by superposing only the most important plane waves. Clearly, if we select a single plane wave, with wave vector $\vec{K}$ in the $\operatorname{direction} \vec{a}$, we will have a version of the plane wave model discussed in the previous section. To do better we propose a Taylor series expansion of $\vec{K}$ near $k \vec{a}$. However, in view of the simple form for the radial integration, it seems unnecessary to restrict the magnitude of the plane waves selected.

Therefore, we wish to expand the angular part of the Fourier transform, $Y_{\ell m}(\hat{K})$, about the direction $a_{a}^{+}$in a Taylor series. It is convenient to rotate our coordinate system to place $\hat{z}$ parallel to $\vec{a}$, so 
that the expansion may be done around the polar axis. If we perform the $m$ sums in equation 3 , using the addition theorem for spherical harmonics, we can get

$$
\begin{aligned}
i^{\ell} h_{\ell}(k r) Y_{\ell m}(\hat{r}) & =\sum_{\ell \prime} i^{\ell^{\prime \prime}} j_{\ell^{\prime \prime}}(k b) \sum_{\ell^{\prime}} 1^{\ell^{\prime}} h_{\ell^{\prime}}(k a) \frac{\left(2 \ell^{\prime \prime}+1\right)(2 \ell+1)}{4 \pi} \\
& * \int Y_{\ell m}(\hat{K}) P_{\ell,}\left(\cos \theta_{a K}\right) P_{\ell \prime \prime}\left(\cos \theta_{b K}\right) d \Omega_{K}
\end{aligned}
$$

Thus only the incoming wave Fourier transform knows that a coordinate system must be rotated; to rotate the spherical harmonic we use

$\left.Y_{\ell m}(\hat{k})\right|_{\vec{z} / / \vec{E}}=\left.\sum_{M=-\ell}^{\ell} Y_{\ell M}(\hat{k})\right|_{\vec{z} / / \vec{a}} R_{M m}^{(\ell)}\left(0,+\theta_{E a}, \pi-\phi_{x \in a}\right)$

The spherical harmonics on the right hand side of this equation are quantized on the $\vec{a}$ axis; the rotation matrix converts the axis of the spherical harmonics from $\hat{z}$ along $\hat{a}$ to $\hat{z}$ along $\hat{\varepsilon}$. Our conventions and definitions for this rotation are discussed in Appendix $B$. To expand $Y_{\ell M}(\hat{k})$ about the new $\hat{z}(a \hat{a})$ axis, we write

$$
Y_{\ell M}(\hat{k})=N_{\ell M} P_{\ell}^{(M)}(\mu) \eta^{M} \quad M \geq 0
$$

$$
=N_{\ell M} P_{\ell}^{(|M|)}(\mu)\left(n^{*}\right)^{M} \quad M<0
$$

where $\mu=\cos \theta, \eta=\sin \theta e^{i \phi}, P_{\ell}^{(M)}(\mu)$ is the $M^{\text {th }}$ derivative of the Legendre polynomial, and $\mathbf{N}_{\ell m}$ is the normalizing coefficient given in Appendix A. The unsubscripted angle variables will refer to $\vec{a}$ as the $\hat{z}$ axis. The Taylor series for $M \geq 0$ is 
$Y_{\ell M}(\hat{k})=\sum_{q=0} \frac{\left[\sin \theta e^{1 \phi q}\right]^{q}}{q !} \sum_{p=0} \frac{(\cos \theta-1)^{P}}{p !}\left[\frac{\partial^{p+q} Y_{\ell M}}{(\partial \mu)^{p}(\partial \eta)^{q}}\right]$

The derivatives are easily managed:

$\left[\frac{\partial^{p+q}}{(\partial \mu)^{p}(\partial \eta)^{q}} Y_{\ell M}\right]=N_{\ell M} P_{\ell}^{(M+p)}(\mu) \frac{M !}{(M-q) !} \eta^{M-q} \underline{\theta}(\ell-p-M)$

We represent the result that these derivatives are zero for $(M+p)>\ell$ by $\underline{\theta}(\ell-p-M)$. When these derivatives are evaluated on the polar axis, only terms with $M-q=0$ will be non-zero; by expanding the derivatives of the generating function ${ }^{10}$ for Legendre polynomials we can show that

$P_{\ell}^{(M+p)}(\mu=1)=\frac{(\ell+M+p) !}{(\ell-M-p) !(2 M+2 p) ! !}$

The Taylor series for $M<O$ is very nearly the same, and we can combine these formulae as

$\left.Y_{\ell M}(\hat{k})|z|\left|a=\sum_{q=-\ell}^{\ell} \sin \right| q\right|_{\theta} e^{i q \phi} N_{\ell q} \sum_{p=0}^{\ell-|q|}(\cos \theta-1)^{p} C_{p q}^{\ell} \delta_{M-q}(\ell-p)$

with

$c_{p q}^{\ell}=\frac{(\ell+|q|+p) !}{(\ell-|q|-p) !} \frac{1}{p !(2|q|+2 p) ! !}$

If we now insert our Taylor series expansion into the rotation, equation (10), the sum on $M$ will contain a single term, $M=q$ : 


$$
\begin{gathered}
\left.Y_{\ell m}(\hat{k})\right|_{z|| \varepsilon}=\sum_{q=-\ell}^{\ell} N_{\ell q}{ }^{(\ell)}\left(0, \theta_{\varepsilon a}, \pi-\phi_{x \varepsilon a}\right) \sin |q|_{\theta} e^{i q \phi} \\
\qquad-\sum_{p=0}^{\ell} C_{p q}^{\ell}\left(\cos \theta_{k}-1\right) p
\end{gathered}
$$

Thus each term in the expansion is associated with a single rotation matrix element.

Inserting this expansion into the summed origin-shift addition theorem, eqn (9), gives

$$
\begin{aligned}
& 1^{\ell} h_{\ell}(k r) Y_{\ell m}(\hat{r})=\sum_{q=-\ell}^{\ell} N_{\ell q}{ }^{(\ell)}\left(0, \theta_{\varepsilon a},^{\pi-\phi} x \in a\right) \sum_{p=0}^{\ell-|q|} c_{p q}^{\ell} \\
& \sum_{\ell "=0}^{\infty} i^{\ell \prime} j_{\ell \prime \prime}(k b) \sum_{\ell=0} i^{\ell '} h_{\ell,}(k a)\left(2 \ell^{\prime}+i\right) \frac{(2 \ell "+1)}{4 \pi} \\
& \int \sin |q|_{\theta} e^{1 q \phi}(\cos \theta-1)^{p} P_{\ell^{\prime}}(\cos \theta) P_{\ell^{\prime \prime}}\left(\cos \theta_{b k}\right) d \Omega
\end{aligned}
$$

Inside the integral we apply the addition theorem for spherical harmonics in reverse to $P_{\ell "}\left(\cos \theta_{b k}\right)$ using $\vec{a}$ as the quantization axis:

$$
\begin{aligned}
& \int \sin |q|_{\theta} e^{i q \phi}(\cos \theta-1)^{p_{P}} P_{\ell}(\cos \theta)\left(\frac{2 \ell "+1}{4 \pi}\right) P_{\ell \prime \prime}\left(\cos \theta_{b k}\right) d \Omega \\
& =\sum_{m "=-\ell "}^{\ell "} Y_{\ell " m "}(\hat{b}) N_{\ell " m "} \quad\left[\int_{0}^{2 \pi} e^{i(q-m ") \phi} d \phi\right] \\
& \int_{-1}^{1} \sin |q|_{\theta}\left(\cos \theta_{k}-1\right)^{p} P_{\ell},(\cos \theta) P_{\ell "}^{m^{\prime \prime}}(\cos \theta) d(\cos \theta)
\end{aligned}
$$


where we have also broken the spherical harmonic down into its component parts (see Appendix A). We note that this use of the addition theorem is equivalent to rotating $P_{\ell "}\left(\cos \theta_{b k}\right)$ to the $\hat{a}$ axis.

The integration of $\phi$ gives $2 \pi \delta_{q m}$, and the sum on $m^{\prime \prime}$ will contain a single term for $m^{\prime \prime}=q$ (or zero if $\left.q>\ell "\right)$. Thus each term in the Taylor series expansion is associated with a single outgoing-wave spherical harmonic.

The $\theta$ integration is slightly more involved. The product of Legendre polynomials and powers of $\sin \theta$ and $\cos \theta$ must be reduced to Legendre polynomials with raised and lowered indices; the integral becomes a series of constants and delta functions relating $\ell$ ' and $\ell "$. The sum on $\ell^{\prime}$ then has only a few terms. If we write

$$
\begin{aligned}
& 4 \pi N_{\ell " q^{\prime}} Y_{\ell " q}(\hat{b}) h_{0}(k a) H_{\ell^{\prime \prime}}^{p q}(k a)=\sum_{\ell^{\prime}} i^{\ell^{\prime}} h_{\ell^{\prime}}(k a)\left(2 \ell^{\prime}+1\right)
\end{aligned}
$$

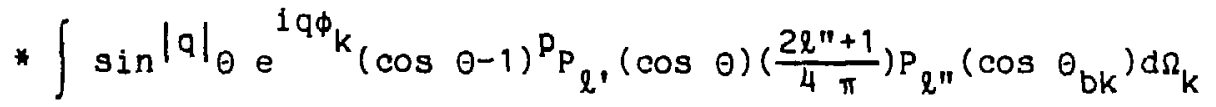

then the origin-shift formula becomes

$$
\begin{aligned}
& i^{\ell} h_{\ell}(k r) Y_{\ell m}(\hat{r})=\sum_{q=-\ell}^{\ell} N_{\ell q} R_{q m}^{(\ell)}\left(0, \theta_{\varepsilon a}, \pi-\phi x \in a\right. \\
& \sum_{p=0}^{\ell-|q|} C_{p q}^{\ell} \\
& \sum_{\ell "}^{\infty} 4 q i^{\ell \prime \prime} j_{\ell "}(k b) Y_{\ell " q}(\hat{b}) N_{\ell " q} h_{0}(k a) H_{\ell "}^{p q}(k a)
\end{aligned}
$$

We give explicit formulas for $H_{\ell \prime \prime}^{p q}(k a), p, q,=0,1,2$ in Appendix $c$. 
This formula is our central result. To compare it with equation 3 note that we can write the Taylor expansion origin shift as

$$
i^{\ell} h_{\ell}(k r) Y_{\ell m}(\hat{r})=\sum_{\ell " q} G_{\ell m \ell " q} i^{\ell "} j_{\ell "}(k b) Y_{\ell " q}(\hat{b})
$$

with the understanding that the quantization axis for $q$ is $\vec{a}$. Thus the Taylor expansion order replaces the magnetic sublevels in the origin shift addition theorem. We will develop this idea more fully in the next section.

\section{MAGNETIC QUANTUM NUMBER EXPANSION}

The mathematical manipulation of the previous section gives little insight into the nature of the Taylor series approximation and hence the value of our result could only be judged after a practical demonstration that a few terms of eqn (21) will suffice to mimic eqn.(3). As reported elsewhere ${ }^{11}$ we have calculated multiple-scattering of photoelectrons based on eqn (21) and we have found rapid convergence. Instead of pursuing that specific application here, we will explore a qualitative picture for the Taylor series expansion which will also provide the connection to the work of Danos and Maximon ${ }^{2}$.

In concluding the previous section we expressed the Taylor series result in a manner which seemed to parallel the original formula, eqn (3). The parallel is imperfect: the quantization axis is constant on both sides of eqn (3) while the spherical harmonics in eqn (22) are expressed on different axes. It is just this difference which leads to the advantages of the Taylor series method. 
It is generally appreciated that the orbital angular momentum quantum number, $\ell$, does not change in rotation matrix elements (eqn (10)), but it is also true that, as discussed by Danos and Maximon, the magnetic quantum number does not change in translation matrix elements when the translation occurs alons the polar $(\hat{z})$ axis. Thus Danos and Maximon accomplish the origin-sifte of an arbitrary spherical wave in three steps. First, they rotate the spherical wave to a coordinate system whose pole coincides with the translation vector. Second, the magnetic-quantum-number conserving translation is applied. In the third and $f$ inal step, they rotate the new spherical waves back to the original coordinate system.

Now we can see the nature of the Taylor series approximation, at least in the index q. As a matter of convenience we rotated the coordinate system in section II, eqn (40), prior to the series expansion. This rotation is precisely the first step of Danos and Maximon. The expansion variable $q$ is the rotated-frame magnetic-quantum number and it survives intact in during the subsequent translation. If we sketch the spherical harmonics in the rotated frame as in figure 1 , then the Taylor approximation is evident: the higher order magnetic sublevels do not overlap the finite range potential. The lowest order approximation, $\mathrm{c}=0$, corresponds to the scattering of the magnetic sublevel which overlaps the strong, central portion of the potential. Higher order sublevels overlap on the weaker regions of the potential until we finally reach a sublevel which does not interact with the potential at all.

With the Taylor series viewed as a magnetic quantum number expansion, we can argue that the number of sublevels required to produce 
adequate results will be related to $\left(r_{0} / a\right)$ if a is the distance between the incident wave source and the center of the potential. Certainly, as the effective radius of the potential decreases or the potential moves away from the source fewer lobes will overlap the potential and a lower order approximation will suffice.

The magnetic quantum number expansion pieture only addresses one of the two dimension approximated in our Taylor series. We speculate on the nature of the approximation governed by the $p$ index as follows. We need only consider origin-shifts along the $\hat{z}$ axis preceded if necessary by a rotation of coordinates. Our procedure in section III may be summarized as Fourier transformation, Taylor series expansion, originshift of each term in the expansion, and finally inverse transformation. Our clue for the nature of the $p$ expansion is the inverse transform integral, eqn. (C5). The expansion variable $(\cos \theta-1)$ is conjugate in the Fourier transform to the operator $[(1 / i k)(\partial / \partial a)-1]$. Thus the $p$ expansion is related to radial correction of the zero-order origin-shift term. While the $q$ index works out from the center of the new origin in an arc laterally towards the edge of the potential, the $p$ index works out along a radius.

As we may show by inserting eqn. (C1) into eqn (21), the zero-order Taylor series term is the addition theorem for an $\ell=0$ spherical wave, i.e. $\exp (i \mathrm{kr}) / \mathrm{krr}$. The addition theorem for higher order spherical waves may be produced by lifting operators applied to the addition theorem for $\ell=0$ waves ${ }^{4}$. Angular derivatives in these lifting operators generate rotation matrix elements corresponding to our $q$ index while radial derivatives generate terms corresponding to our $p$ index ${ }^{12}$. This relation is further borne out in the equivalence drawn between the 
lifting operator picture and the Taylor series for the particular application to photoelectron scattering in ref. 11, Appendix $A$.

\section{CONCLUSION}

We have presented a nes formula for the translation of screened spherical waves, a series adapted for truncation, which allows the accuracy of curved-wave corrections to be continuously traded for ease of calculation. Furthermore we have related one of the expansion variables to magnetic quantum numbers in a frame rotated to place the pole along the translation axis.

Our form for the origin-shift addition theorem should prove useful to a number of physical problems. Even restricting our attention to electron scattering in solids leaves applications to theories of X-ray absorption fine structure, electron diffraction, electron energy loss fine structure, and our original interest, photoelectron scattering. Our application of equation (21) to photoelectron scattering is discussed in ref 11 . 
APPENDIX A. OUR SPHERICAL HARMONICS

The properties of spherical harmonics are, of course, well known, but the phase conventions do vary, especially between older works and between more mathematical and more physical works. We will use the most common "Condon-Shortley" definition":

$Y_{\ell|m|}(\theta, \phi)=N_{\ell m}{ }^{P}{ }_{\ell}^{|m|}(\cos \theta) e^{i|m| \phi}$,
$Y_{\ell-|m|}(\theta, \phi)=N_{\ell m}{ }^{|m|}(\cos \theta) e^{-i|m| \phi}$,

with

$N_{\ell m}=\left[\frac{2 \ell+1}{4 \pi} \frac{(\ell-|m|) !}{(\ell+|m|) !}\right]^{1 / 2}$ for $m<0$ and the same times $(-1)|m|$ for $m \geq 0$

We use

$P_{\ell}^{m}(\cos \theta)=\sin \theta \frac{d^{m} P_{\ell}(\cos \theta)}{(d \cos \theta)^{m}}$

where $P_{Q}(\cos \theta)$ is the Legendre polynomial; this choice for the associated Legendre polynomial agrees with Nozawa ${ }^{4}$ and Pendry ${ }^{8}$, but and Abramowitz and $\operatorname{stegin}^{13}$ add a factor of $(-1)^{\text {m }}$. 
APPENDIX B. COORDINATE SYSTEMS, EULER ANGLES, AND ROTATIONS

The rotation matrix method is powerful but prone to error from factors of $(-1)^{\mathrm{m}}$ arising from misinterpretation of the axes. To avoid confusion, we detail our convention on coordinate relations and notation in this appendix.

Our initial coordinate system has its origin on the photoemitting atom with its $\hat{z}$ axis along the electric vector $\hat{\varepsilon}$. For our purposes the remaining degree of freedom is arbitrary. For surface problems it is most convenient to place the $y$ axis in the surface plane. Our coordinate systems are always right handed. We refer to this initial system as $z|| \varepsilon$.

We use the Euler angle convention of Messiah. ${ }^{14}$ Positive angles mean rotation as a right-handed screw advancing on the axis of rotation ( $r$ ight-hand rule). The rotation $\mathrm{R}(a, \beta, \gamma)$ begins with a rotation by $a$ about the initial $z$ axis. This a rotation repositions the initial $y$ axis so that it may serve as the $B$ rotation axis. The $B$ rotation proceeds about the intermediate, repositioned y axis; it carries the initial $z$ axis into the final $z$ axis. The $\gamma$ rotation uses the final $z$ axis for rotation.

A vector, $\vec{a}$, in the $z|| \varepsilon$ system has a polar angle (latitude) measured down from the $+z$ axis of $\theta_{\varepsilon a}$; its azimuth (longitude) measured from the $+x$ axis is $\phi_{x \in a}$. The three subscripts of the azimuth are the reference axis, polar axis and vector label, respectively. More generally, if the reference axis and polar axis are not perpendicular, the azimuth is measured from the half plane bordered by the pole and containing the reference axis. We design the rotation which takes the 
$z|| \varepsilon$ system into the $z|| a$ system to use $\hat{\varepsilon}$ as the reference axis in the new system. To accomplish this we use $R\left(\phi_{x \in a}, \theta_{\varepsilon a}, \pi\right)$ where the final rotation by $\pi$ brings the new $+x$ axis into the same half plane as $\hat{\varepsilon}$. The $+y$ axis of the $z$ |la system is parallel to the vector cross product $\hat{a} \hat{x} \hat{\varepsilon}$.

Spherical harmonics in the z/le system may be written as a linear combination of spherical harmonics in the $z$ ||a system with unchanged orbital angular momentum:

$|\ell m\rangle=\left|\ell, m^{\prime}\right\rangle\left\langle\ell m^{\prime}|R(\alpha \beta \gamma)| \ell m\right\rangle$

or

$Y_{\ell m}(\hat{K})\left|\hat{z} / \hat{\varepsilon}=\sum_{m^{\prime}=-l}^{\ell} Y_{\ell m}(\hat{K})\right|_{\hat{z} / / \hat{a}} R_{m^{\prime} m}^{(l)}(\alpha \beta Y)$

The rotation must take us back from $z|| a$ to $z|| \varepsilon$ : we must use the inverse of the $z|| \varepsilon$ to $z||$ a rotation. We have."

$$
[R(\alpha \beta \gamma)]^{-1}=R(-\alpha,-\beta,-\gamma)
$$

and the required rotation matrix elements are $R_{m}^{(l)}\left(-\pi,-\theta_{\varepsilon a},-\phi_{x \in a}\right)$; equivalently we may use $R_{m^{\prime} m}^{l}\left(0, \theta_{\varepsilon a}, \pi-\phi_{x \varepsilon a}\right)$.

Once we have moved into the $z$ lla system, another scattering potential along bond vector $\vec{b}$ will have angular coordinates $\left(\theta_{a b}, \phi_{E a b}\right)$ and the necessary rotation matirix elements wili be $R_{m^{\prime} m}^{l}\left(0, \theta_{a b}, \pi-\right.$ $\left.\phi_{\varepsilon a b}\right)$. Note that if the coordinates of the vectors $\hat{\varepsilon}, \hat{a}$, and $\hat{b}$ are known along an arbitrary (e.g. crystalline) axis system, then the angles 
required for the rotation matrix construction may be derived from the dot product $\varepsilon \cdot a=\cos \theta_{\varepsilon a}$ and a relation of spherical trigonometry:

$\cos \theta_{\varepsilon b}=\cos \theta_{\varepsilon a} \cos \theta_{a b}+\sin \theta_{\varepsilon a} \sin \theta_{a b} \cos \phi_{\varepsilon a b}$

with the sign of $\phi_{\varepsilon a b}$ equal to the sign of $\vec{b} \cdot(\vec{a} \times \hat{E})$. 
APPENDIX $C:$ ON THE TAYLOR-SERIES INTEGRALS

To calculate the origin-shift addition theorem coefficients with the Taylor series formula we need to evaluate

$$
\begin{aligned}
& \mathrm{H}_{\ell}^{\mathrm{pq}}(\mathrm{ka})=\sum_{\ell,} \mathrm{d}_{\ell},(\mathrm{ka})\left(2 \ell^{\prime}+1\right) \\
& \frac{1}{2} \int_{-1}^{1} \sin ^{q} \theta(\cos \theta-1)^{p} P_{\ell},(\cos \theta) P_{\ell}^{q}(\cos \theta) d \cos \theta
\end{aligned}
$$

We have introduced $d_{\ell}(k a)$ as the non-asymptotic component of the spherical Hankel functions, i.e. $h_{\ell}(k r)=d_{\ell}(k r) \exp (i k r) / i k r$. We group the sum and integral together to emphasize the restrictions the integral will place upon the range of the sum. We work out individual cases through second order; we also give recursive and explicit forms for nigher order.

The basic method is to express the product of coordinates and a Legendre polynomial as a series of Legendre polynomials with raised and lowered indices using ${ }^{10}$

$(2 \ell+1) \sin \theta P_{\ell}^{m+1}(\cos \theta)=(\ell+m)(l+m+1) P_{\ell-1}^{m}(\cos \theta)-(\ell-m)(l-m+1) P_{\ell+1}^{m}(\cos \theta)$

and

$(2 \ell+1) \cos \theta P_{\ell}^{m}(\cos \theta)=(\ell-m+1) P_{\ell+1}^{m}(\cos \theta)+(\ell+m) P_{\ell-1}^{m}(\cos \theta)$

This will leave the orthogonality integral for Legendre polynomials: 
$\frac{1}{2} \int_{-1}^{1} P_{\ell}(\cos \theta) P_{\ell}(\cos \theta) d \cos \theta=\frac{\delta \ell \ell}{\left(2 \ell^{\prime}+1\right)}$

and the sum on $\ell$ ' may be completed.

The specific cases we require are:

1) $p=q=0$. We Immediately get the orthogonality condition and

$H_{\ell}^{00}(k a)=d_{\ell}(k a)$

ii) $p=1, q=0$. The $\cos \theta$ recursion gives

$H_{\ell}^{10}(k a)=\left(\frac{1}{2 \ell+1}\right)\left[(\ell+1) d_{\ell+1}(k a)+\ell d_{\ell-1}(k a)-(2 \ell+1) d_{\ell}(k a)\right]$

iii) $p=0, q=1$. The $\sin \theta$ recursion gives

$H_{\ell}^{01}(k a)=\frac{\ell(\ell+1)}{(2 \ell+1)}\left[d_{\ell-1}(k a)-d_{\ell+1}(k a)\right]$

1v) $p=2, q=0$. The $\cos \theta$ recursion must be applied twice to give

$$
\begin{aligned}
\mathrm{H}_{\ell}^{20}(k a)= & \left(\frac{1}{2 \ell+1}\right)\left[\left[\frac{(\ell+1)(\ell+2)}{2 \ell+3}\right] d_{\ell+2}(k a)+\left[\frac{(\ell+1)^{2}}{(2 \ell+3)}+\frac{\ell^{2}}{(2 \ell-1)}\right] d_{\ell}(k a)+\right. \\
& {\left.\left[\frac{\ell(\ell-1)}{(2 \ell-1)}\right] d_{\ell-2}(k a)-2(\ell+1) d_{\ell+1}(k a)-2 \ell d d_{\ell-1}(k a)+(2 \ell+1) d_{\ell}(k a)\right\} }
\end{aligned}
$$


v) $p=1, q=1$. First the associated Legendre polynomial is reduced to $m=0$ with the $\sin \theta$ recursion, then the $\cos \theta$ recursion is applied. Then

$$
\begin{array}{r}
H_{\ell}^{11}(k a)=\frac{\ell(\ell+1)}{(2 \ell+1)}\left[\frac{(\ell-1)}{(2 \ell-1)} d_{\ell-2}(k a)-d_{\ell-1}(k a)+\frac{(2 \ell+1)}{(2 \ell-1)(2 \ell+3)} d_{\ell}(k a)\right. \\
\left.+d_{\ell+1}(k a)-\frac{(l+2)}{(2 \ell+3)} d_{\ell+2}(k a)\right]
\end{array}
$$

vi) $p=0, q=2$. From the $\sin \theta$ recursion for $m=1$ we have

$$
\begin{aligned}
H_{\ell}^{02}(k a)= & \frac{(\ell+1)(\ell+2)}{(2 \ell+1)}\left[\frac{(\ell-1) \ell}{(2 \ell-1)}\right]\left[d_{\ell-2}(k a)-d_{\ell}(k a)\right]- \\
& \frac{(\ell+1)(\ell+2)}{(2 \ell+3)}\left[\frac{(\ell-1) \ell}{(2 \ell+1)}\right]\left[d_{\ell}(k a)-d_{\ell+2}(k a)\right]
\end{aligned}
$$

To check these results we employ a method demonstrated by Nozawa 4 to obtain recurrence relations for quantities $11 \mathrm{ke} \mathrm{H}_{l}^{\mathrm{pq}}$. We consider

$J_{\ell}^{p q}(k a)=\sum_{\ell,}(2 \ell+1) 1^{\ell^{\prime}} j_{\ell},(k a) \frac{1}{2} \int_{-1}^{1} \sin ^{q} \theta(\cos \theta-1) P_{P^{\prime}}(\cos \theta) P_{\ell}^{q}(\cos \theta) d \cos \theta$

This is $h_{0}(k a) H_{\ell}^{p q}$ with the spherical Bessel function $j_{Q}(k a)$ replacing $\mathrm{h}_{\ell}(k a)$. If we avoid complex conjugation, we may use results derived for $\mathrm{J}_{\ell}^{\mathrm{pq}}$ for $\mathrm{H}_{\ell}^{\mathrm{pq}}$ by replacing $i^{\ell} j_{\ell}(\mathrm{ka})$ by $d_{\ell}(k a)$. We do not of fer a proof of this convenience; we find, however, that the results are correct. The sum on $\ell$ ' in $J_{\ell}^{p q}$ is the Bauer formula, eqn (1), giving

$$
J_{\ell}^{p q}(k a)=\frac{1}{2} \int_{-1}^{1} \sin ^{q} \theta(\cos \theta-1)^{p} e^{i k a \cos \theta} P_{\ell}^{q}(\cos \theta) d \cos \theta
$$


We may immediately deduce a recurrence in $p$ :

$J_{\ell}^{p+1, q}(k a)=\left(\frac{1}{i k} \frac{\partial}{\partial a}-1\right) J_{\ell}^{p q}(k a)$

A derivation for raising $q$ is more involved. We consider the integral

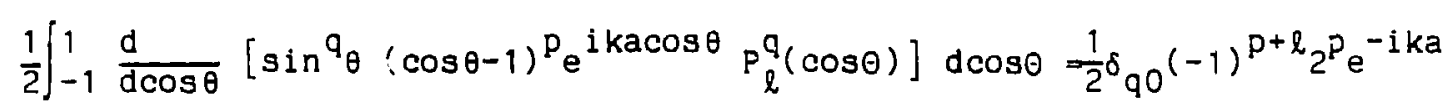

A suitable definition of associated Legendre functions gives

$P_{\ell}^{q}(\cos \theta)=(-1)^{q} \frac{(\ell+q) !}{(\ell-q) !} P_{\ell}^{-q}(\cos \theta)$

Thus

$\sin ^{q} \theta P_{\ell}^{q}(\cos \theta)=(-1)^{q} \frac{(l+q) !}{(l-q) !} P_{\ell}^{(-q)}(\cos \theta)$

where we recall that the parenthetical supe:script $q$ indicates $q^{\text {th }}$ derivative; for negative $q$ we use

$$
P_{\ell}^{(-q)}(x)=\frac{1}{2^{l} \ell !}\left(\frac{d}{d x}\right)^{\ell-q}\left(x^{2}-1\right)^{\ell}
$$

Our integral becomes

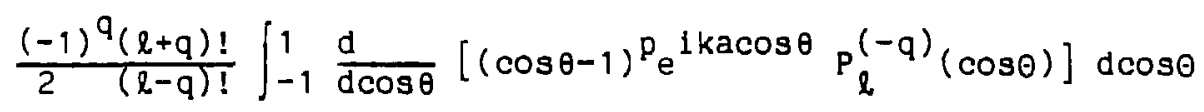




$$
=\delta_{q 0}(-1)^{p+\ell} 2^{p} e^{-i k a}
$$

Applying the derivatives gives, for $q$ not zero:

$$
J_{\ell}^{p, q+1}(k a)=\frac{(\ell+q+1)(\ell-q) J_{\ell}^{p q}(k a)-p J_{\ell}^{p+1, q}(k a)}{i k a}
$$

The cosine recursion for associated Legendre polynomials and the result of operation on $\mathrm{J}_{\ell}^{\mathrm{pq}}$ with $(1 / \mathrm{k})(\partial / \partial \mathrm{a})$ gives another useful relation:

$J_{\ell}^{p+1, q}+J_{\ell}^{p q}=(\ell+q) J_{\ell-1}^{p q}+(\ell-q+1) J_{\ell+1}^{p q}$

and the sine recursion gives

$$
(\ell-q)\left[J_{\ell}^{p+1, q}+J_{\ell}^{p q}\right]=(\ell+q) J_{\ell-1}^{p q}-J_{\ell}^{p, q+1}
$$

To collect these results we give a strategy for constructing a table of $H_{\ell}^{p q}$. Equation $(C 1)$ and the recursion for $d_{\ell}(k a)$ generates the first row:

$H_{\ell+1}^{00}(k a)=H_{\ell-1}^{00}(k a)-\frac{(2 \ell+1)}{1 k r} H_{\ell}^{00}(k a)$

The raising operator for $q$, eqn. (C3), gives the rows $q=1, \tau$; Equation (C4) raises $p$ using the current contents of the table and eqn. (C3) again applies. 
Finally, an explicit formula will be useful for discussing the convergence of the Taylor series. The raising operator relation for $p$ may be repeatedly applied to give

$J_{\ell}^{p q}=\left(\frac{1}{i k} \frac{\partial}{\partial a}-1\right)^{p} \frac{1}{2} \int_{-\pi}^{\pi} \sin ^{q} \theta e^{i k a \cos \theta} P_{\ell}^{q}(\cos \theta) d \theta$

The remaining integral is proportional to an integral discussed by Nozawa ${ }^{4}$. If we recall that the associated Legendre polynomial with maximum magnetic quantum number is simply related to $\sin \theta$ :

$P_{q}^{q}(\cos \theta)=\frac{(2 q) !}{2^{q} q !} \sin ^{q} \theta$.

we will consult Nozawa's equation 6.1 :

$\frac{1}{2} \int_{-1}^{1} d \mu e^{1 \mu k a} P_{q}^{q}(\mu) p_{\ell}^{q}(\mu)=\frac{(\ell+q) !}{(\ell-q) !}(2 q-1) ! ! \frac{i^{\ell} j_{\ell}(k a)}{(i k a)^{q}}$

and write

$J_{\ell}^{p q}(k a)=\left(\frac{\partial}{i k \partial a}-1\right)^{p} \frac{(\ell+q) !}{(\ell-q) !} \frac{i^{\ell} j_{\ell}(k a)}{(i k a)^{q}}$.

Returning now to spherical Hankel functions we claim that

$\frac{e^{i k a}}{1 k a} H_{\ell}^{p q}=\left(\frac{1}{i k} \frac{\partial}{\partial a}-1\right)^{p} \frac{(\ell+q) !}{(l-q) !} \frac{i^{\ell} h_{\ell}(k a)}{(i k a)^{q}}$

Notice that the exponential dependence cancels, taking with it the constant part of the differential operator:

$\left(\frac{1 \partial}{i k \partial a}-i\right) \frac{i^{\ell} h_{\ell}(k a)}{(i k a)^{q}}=e^{i k a}\left[\left(\frac{1}{1 k} \frac{\partial}{\partial a}\right) \frac{d_{\ell}(k a)}{(1 k a)^{q+1}}\right]$

Then we have

$H_{\ell}^{p q}=i k a\left(\frac{1}{i k} \frac{\partial}{\partial a}\right)^{p} \frac{d_{\ell}(k a)}{(i k a)^{q+1}} \frac{(l+q) !}{(\ell-q) !}$ 
We may substitute the series definition of $d_{\ell}(k a)$ to get

$H_{\ell}^{p q}(k a)=\frac{(l+q) !}{(\ell-q) !}(-1)^{q} q^{q+p} \sum_{t=0}^{\ell} \frac{(\ell+t) !}{t !(\ell-t) !} \frac{(t+p+q) !}{(t+q) !}\left(\frac{i}{2 k a}\right)^{t+q+p}$

or we can retain $d_{\ell}(k a)$ and apply the derivatives to the products

$$
H_{\ell}^{p q}(k a)=\frac{(l+q) !}{(l-q) !} \frac{(-1)^{p}}{(i k a)^{q+p}} \frac{p !}{q !} \sum_{s=0}^{p} \frac{(q+p-s) !(k a)^{s}}{s !(p-s) !} \frac{\partial^{s} d_{\ell}(k a)}{\partial(k a)^{s}}
$$




\section{ACKNOWLEDGEMENTS}

This work was supported by the Director, Office of Energy Research, Orfice of Basic Energy Sclences, Chemieal Sciences Division of the U.S. Department of Energy under Contract No. DE-AC03-76SF00098. 


\section{REFERENCES}

1. P.A. Lee, P.H. Citrin, P. Elsenberger, and B.M. Kincaid, Rev. Mod. Phys. 53, 769 (1981).

2. M. Danos and L.C. Maximon, J. Math. Phys. 6, 766 (1965).

3. C. Cohen-Tannoudji, B. Diu, F. Laloë, Quantum Mechanics, John Wiley and Sons, New York, 1977.

4. R. Nozawa, J. Math. Phys. I, 1841 (1966).

5. J.C. Slater, The Self Consistent Fleld for Molecules and Solids, McGraw-Hill, 1974.

6. W. Kohn and N. Rostoker, Phys. Rev. 94, 1111 (1954); J. Korringa, Physica, 13, 392 (1947).

7. J.A. Gaunt, Phil. Trans Roy. Soc. (London) A228, 151 (1924).

8. J.B. Pendry, Low Energy Electron Diffraction, Academic Press, London, (1974).

9. If $\ell^{\prime \prime}+\ell^{\prime}+2$ is even and $a>b$ then a contour along the real axis may be closed up to encircle one pole at $(k+i \delta / 2 k)$. See, for example, Goertzel, G. and Tralli, N. Some Mathematical Methods of Physics, 1960, p. 172. For $\ell "+\ell$ odd we may not use this method. Note, however, that for asymptotically large $|\vec{a}|$, the integral becomes independent of $\ell^{n}$ and is not zero: we presume it has the same form as the even case with no factors like $(-1)^{\ell+l "}$. This is evidently correct since the addition theorem may be derived by independent means.

10. E. Butkov, Mathematlcal Physics, Addison Wesley, Reading, MA (1968). 
11. J.J. Barton and D.A. Shirley, "Small Aton Approximations for Photoelectron Scattering in the Intermediate Energy Range" LBL-18693, and submitted to Phys. Rev. A., and Chapter 4

12. J.J. Barton and D.A. Shirley, "Curved Wavefront Corrections for Photoelectron Scattering," LBL-18692, and subnitted to Phys. Rev. B., and Chapter 2

13. M. Abramowitz and I.A. Stegun, Handbook of Mathematical Functions, National Bureau of Standards, Appl. Math. Serv. 55 (1964).

14. A. Messiah, Quantum Mechanics, John Wiley and Sons, New York (1958). 


\section{FIGURE CAPTION}

Figure 1. Schematic illustration of the magnetic quantum number expansion interpretation of eqn. (21). A polar plot of the four lowest magnetic sublevels of a $\ell=7$ spherical harmonic is superimposed upon a circle whose radius represents the effective radius $r_{0}$ of a nearest neighbor potential. The line connecting the incident wave source and the potential origin is used for the spherical harmonic polar axis and only the region of angles near the pole is plotted. The angle functions have been rescaled to place their first maxima on the same radius. The $m=0$ sublevel (solid line) is seen to overlap the strong central portion of the potential, while the $m=1$ lobes (dotted line) peaks further from the axis. The $m=2$ lobes (dot-dash lines) only intercept the $f$ ar edges of the potential and the $m=3$ level (dashed lines) completely missed the mark. 


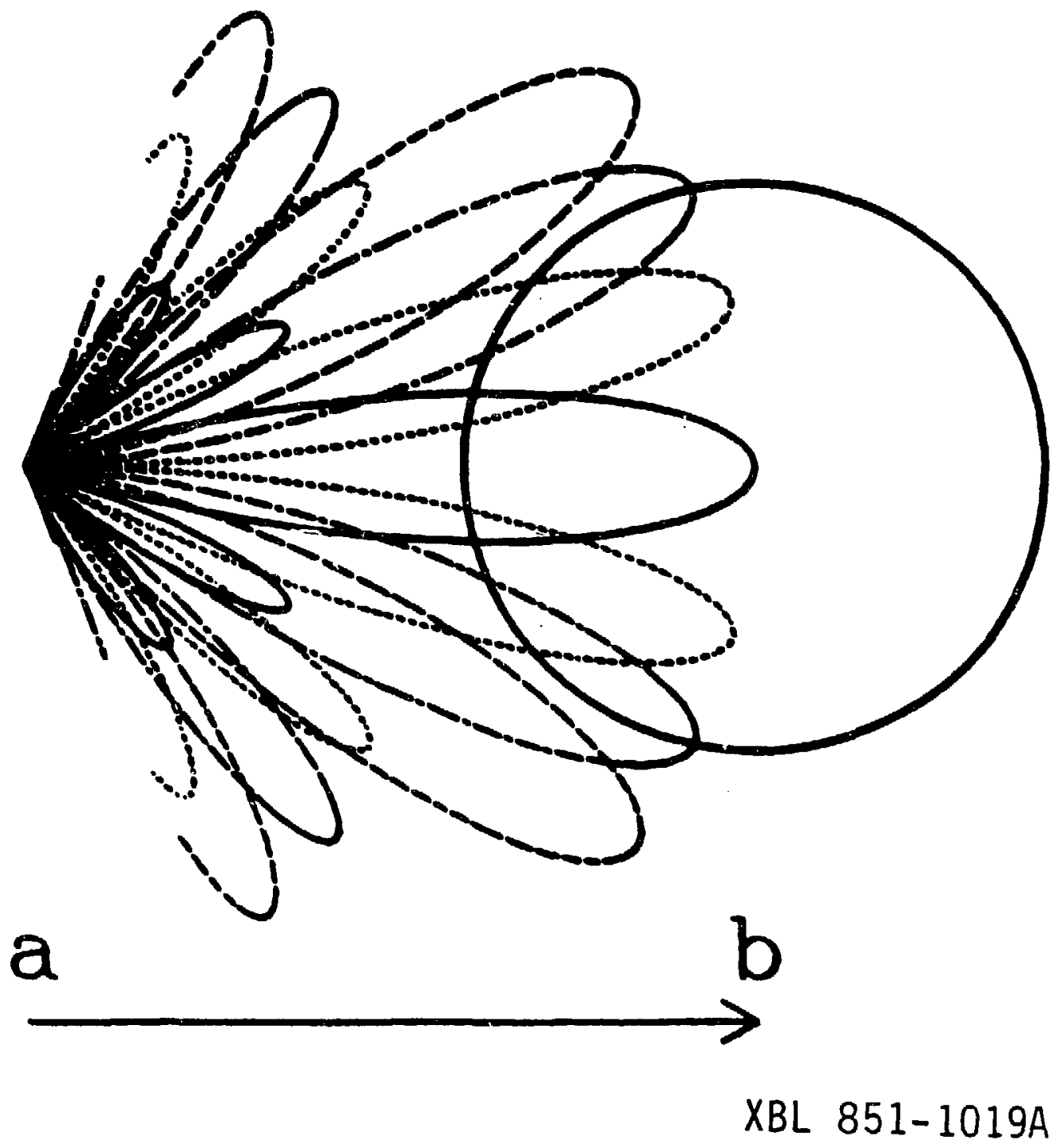

Figure 1 
CHAPTER 4:

SMALL ATOM APPROXIMATIONS FOR PHOTOELECTRON SCATTERING

IN THE INTERMEDIATE ENERGY RANGE

ABSTRACT

Flve approximate models for describing the scattering of spherical waves by central potentials are explored. The point-scattering model introduced by Lee and Pendry (Phys. Rev. B, 11,2795,(1975)) allows a short-range potential to be close to the source; a new homogeneous wave model lifts the restriction on the potential diameter, but requires asymptotic incident waves. The popular plane-wave model requires both an infinitesimal diameter potential and incident waves at their asymptotic limit. For realistic potentials at near-neighbor separations, none of these models is adequate: even a hybrid model combining features of the point-scattering and homogeneous-wave methods does not allow for amplitude variation across the potential. The fifth small atom model is based on a Taylor series, magnetic quantum number expansion of the addition theorem for screened spherical waves. This Taylor series approximation has the homogeneous-wave model as its zeroorder term and the exact spherical wave scattering process as its limit. Multiple-scattering equations for Angle-Resolved Photoemission Extended Fine Structure (ARPEFS) are derived and the effectiveness of these approximations are compared. We demonstrate that while the plane-wave model is reasonably accurate for near-180 backscattering, small angle 
scattering requires the curved wavefront corrections available in the T'aylor series expansion method. 


\section{INTRODUCTION}

The theoretical description of electron scattering in solids for intermediate energies from 100 to $1000 \mathrm{eV}$ faces a peculiar challenge: simple physical models for the scattering process are surprisingly accurate, while more sophisticated calculations become intractable. The striking success of single-scattering, plane-wave models for the qualitative description of Angle-Resolved Photoemission Extended Fine Structure (ARPEFS) ${ }^{1}$ and of Extended X-ray Absorption Fine Structure (EXAFS $^{2}$ has led to satisfactory empirical analysis of these measurements, but multiple-scattering, spherical wave calculations are too complex for routine quantitative analysis cases where the simple model fails.

The two crucial theoretical issues--spherical vs. plane-wave scattering and multiple versus single scattering--are entwined. First, we must decide if the curvature of the wave emanating from one ion core will influence the scattering of that wave by an adjacent potential. If we may neglect the curvature, then the scattering of electrons in a solid reduces to a series of plane-wave calculations, connected only through the wave phase and incident direction. ${ }^{3}$ If, on the other hand, the curvature is important, more sophisticated calculations are required. For single scattering of $l=1$ photoelectrons we have previously derived the required equations. Unfortunately the outgoing scattered wave from the first scattering event will always contain much higher angular momentum partial waves, requiring more sophisticated 
equations and leading to larger corrections. Thus if double scattering is important, we certainly would expect the wave curvature to be important.

This leads us to the second central issue in electron scattering: the role of multiple scattering. The varlous experimental processes address different aspects of this issue. The results of Low Energy Electron Diffraction (LEED) calculations ${ }^{5}$ are clear: multiple scattering is important. Theoretical work with photoelectron scattering ${ }^{6}$ and more recent work on $x$-ray absorption edges ${ }^{7}$ have utilized the relative simplicity of the photoelectron scattering process to show that at intermediate energies only forward scattering leads to multiple scattering events at a level that cinnot be neglected. Finally in EXAFS the photoelectron returns to the abcorhing atom: in the process a large number of high angular-momentum waves are backscattered to the abso. z:r. Thus our previous conclusion that double scattering would require curved wave calculations is contradicted by evidence 8,9 that a plane-wave model contains the essential character of the EXAFS phenomenon; we might be further tempted to extrapolate the empirical evidence and ignore wavefront curvature for all scattering at high energy. ${ }^{3}$ Any correct theoretical procedure must be compatible with all of these observations. In a previous paper, we explored the wavefront curvature question for single scattering of $\ell=1$ photoelectrons. Two important conclusions from that work are 1) curved wave corrections are much smaller for backscattering than they are for forward scattering, and ii) only the backscattering corrections get smaller at high energy; the forward 
scattering corrections do not fall asymptotically with large $k$. Because the studies cited above already demonstrate the importance of forward scattering, and because the once-scattered wave will contain angular momenta much greater than $\ell=1$, we are led to investigate curved wavefront corrections to multiple scattering in the intermediate energy range.

This would seem to be a rather straightforward project. We have after all an impressive foundation in the low energy region from LEED theory, ${ }^{5}$ photoelectron diffraction ${ }^{10}$, and, more recently, near edge $x-$ ray absorption. 11 Indeed, Tong and co-workers have extended their photoelectron diffraction calculations into the intermediate energy range, ${ }^{10}$ and their results have provided indications of the importance of multiple scattering. These calculations are not, however, simple extensions of the plane wave model; they are full curved-wave treatments and consequently--for a reason we now discuss--much more expensive than low energy multiple scattering calculations.

The origin of the difficulty in applying multiple scattering curved wave calculations at intermediate energies is that the maximum angular momentum in a scattered wave leaving a scattering atom increases with electron wavenumber. The maximum angular momentum, $\ell_{\max }$ scattering from a potential may be related to its range, $r_{0}$, by!

$$
k r_{0}=\sqrt{l_{\max }\left(l_{\max }+1\right)}=l_{\max }
$$


As the energy--and hence the number of important scattered waves-increases, the number of numerical operations also increases. For planewave models this increase is manageable because each scattering event depends only linearly on $\ell_{\max }$. On the other hand, the time required for exact curved wave calculations are proportional to at least $\left(l_{\max }\right)^{4}$, prohibiting even exploratory multiple scattering calculations to be attempted. (Also note that approximate curved wave calculations based on a small, fixed maximum angular momentum will be incorrect above some energy given by eqn. (1)).

Simply from the success of plane-wave models of electron scattering we may conclude that another physical approximation must exist that would allow accurate introduction of curved wave corrections with more modest effort than the exact theory requires. In this paper we seek such an approximation by examining five approximate models for the scattering of spherical waves from central potentials:

1) point scattering,

ii) homogeneous wave scattering,

iii) plane-wave,

iv) renormalized homogeneous wave,

v) Taylor series-magnetic quantum number expansion.

The first four models were initially motivated by existing angularmomentum expansions known as addition theorems. We discuss the approximations necessary for each model and outline the physical problems to which they apply. The fifth small atom approximation is based on a new angular-momentum series expansion ${ }^{13}$; it contains 
important curved wave corrections not included in any of the first four models and it provides for orderly development of higher order corrections.

In section II we discuss electron scattering of spherical waves by the partial wave method and exact scattering of spherical waves in the 100-1000 eV range. Physically motivated small atom approximations to the spherical wave formula are reviewed in section III, and we examine some of the contributions to the success of the plane-wave model. The remaining sections concern the fifth, more general small atom model. Section IV applies the Taylor series expansion for the spherical wave scattering to (1s) initial state photoelectron scattering. Section $V$ discusses spherical wave scattering factors which govern the convergence of the Taylor series and Section VI illustrates the convergence for a single example and describes an intuiti $\epsilon$ picture of the Taylor series terms. We conclude in section VII with some remarks on further applications. 
II. SPHERICAL WAVE SCATTERING AND NOZAWA'S GAUNT INTEGRAL SUMMATION

To provide the background for our development of approximate electron scattering formulae, we will set up the electron scattering problem, review Nozawa's origin-shift addition theorem ${ }^{14}$ for spherical waves, and discuss the difficulty with this approach.

Our scattering system consists of a point source of electrons and a lattice of non-overlapping central potentials (the muffin-tin model ${ }^{5}$ ). This model approximates a number of physical problems. If the lattice represents a surface system and the point source is a screened photoemitter, then we have a photoelectron diffraction or Angle-Resolved Photoemission Extended Fine Structure model. If the lattice is a bulk material or surface system, the point source is again a screened photoemitter, and we calculate an integrated photoabsorption cross section, then we have an Extended $X$-ray Absorption Fine Structure (EXAFS) model. If the lattice is a surface, but the point source is a once scattered electron from an incoming plane wave, we have part of a model for multiple scattering, Low Energy Electron Diffraction (a sum over all scattering potentials excited by the plane wave is required to complete the LEED problem). For numerical calculation and physical discussion, the photoelectron diffraction (ARPEFS) case is the simplest problem because the scattered wave is directly detected. We shall concentrate on this problem.

If we consider a model which consists of non-overlapping potentials, we may calculate the multiple scattering by combining a general description for a spherical wave scattering from a potential 
with a prescription for enumerating all significant scattering paths. Because we wish to describe photoelectron diffraction in the intermediate energy range (ARPEFS), we will concentrate on the scattering of photoelectrons originating from a (1s) level of an atom in an ordered and oriented system. We will also choose our examples to correspond to a recent ARPEFS experiment ${ }^{1}$ on the $c(2 \times 2) S / N i(001)$ system. When the angle-resolved cross section for $S(1 s)$ photoemission is measured in such a system, the intensity oscillates with energy demonstrating interference among channels describing the possible paths to the detector. 15 Most of the interference occurs between waves describing direct and single-scattered photoemission, but forward scattering of the single-scattered electrons must also be included to predict the interference amplitude correctly. 10 A more complete discussion of the physics of photoelectron diffraction can be obtained elsewhere.,15,16 For the purposes of this paper we note that:

1) dipole selection rules predict that a single p type $(l=1)$ continuum orbital is populated through photoabsorption by a (1s) initial orbital,

ii) by selecting the $\hat{z}$ axis of our coordinate system parallel to the electric vector of a polarized photon beam, the photoelectron angular distribution can be written as proportional to $Y_{10}(\hat{r})=(3 / 4 \pi)^{1 / 2} \cos \theta$,

iii) in most materials, the photoion may be considered fully screened on the absorbing atom, and 
iv) both the photoabsorbing atom's phase shift of the continuum wave and the total atomic cross section appear equally in all terms describing the final amplitude and thus do not affect the oscillations. Thus the oscillations may be isolated by normalization to form a function $x(k)=I(k) / I_{0}(k)-1$ as in EXAFS, but $x(k)$ contains no source-atom phase shift, in contrast to EXAFS.

With these ideas in mind, we may write the important part of the outgoing wavefunction as an $\ell=1$ spherical wave

$$
\Psi_{0}(\hat{r})=\operatorname{in}_{1}(k r) Y_{10}(\hat{r})
$$

We refer to this wavefunction as the "direct" wave.

We will use the scattering geometry deplcted in Figure 1 . We use $\vec{r}$ as a general position vector, and $\vec{k}$ as the propagation vector of the electron, with $k$ giving the electron wave number. We define the scattering potential positions by $\vec{a}, \vec{b}, \vec{c} \ldots$ for the first, second, third and so on, scattering events. These latter vectors connect atoms: they are "bond" vectors, not position vectors. The vector $\vec{R}$ is the position of the angle resolving detector. Our convention and notation for spherical harmonics, $Y_{\ell m}$, are given in Appendix A of ref 13. For brevity we use the notation $Y_{\ell m}(\hat{r})$, where $\hat{r}=\vec{r} / r$. The functions $j_{\ell}(k r)$ and $h_{\ell}(k r)=h_{\ell}^{(1)}(k r)$ are spherical Bessel functions as defined by, for example, Pendry ${ }^{5}$, Appends $x$ A. 
Our discussion of the small atom approximations will consider the single and double scattering of $\ell=1$ photoelectron waves. A single scattering event will have an incident $\ell=1$ wave (eqn. (2)), but the second event in double scattering will include all angular momenta characteristic of the multiple scattering problem. Thus higher order scattering can be built up by repeating the steps in the second part of our double scattering equations.

To better understand the small atom models, we will first briefly review the potential scattering of plane and spherical waves. If a plane wave is incident upon the potential, the expansion in an angular momentum series is well known:

$$
e^{i \vec{k} \cdot \vec{r}}=\sum_{\ell m^{n}} 4 \pi i^{\ell n} j_{\ell n}(k b) Y_{\ell " m n}(\hat{b}) Y_{\ell " m n}^{*}(\hat{k})
$$

where $\vec{k}=k \hat{a}$ and $\vec{r}=\vec{a}+\vec{b}$. The partial wave method ${ }^{17}$ instructs us to expect an outgoing spherical wave, $i^{\ell} h_{\ell}(k r) Y_{\ell m}(k r)$, proportional to each regular spherical wave incident on the potential; the complex proportionality factor, $T_{\ell}$, is derived from the partial wave phase shifts, $\delta_{\ell}(k)$, and has both a scattering amplitude and wave phase shift:

$T_{\ell}(k)=1 \sin \delta_{\ell} e^{1 \delta_{\ell}}=\frac{1}{2}\left(e^{12 \delta_{\ell}(k)}-1\right)$ 
$\Psi_{\vec{a}}(\vec{b})=\sum_{\ell " m^{\prime \prime}} 4 \pi i^{\ell " h_{\ell "}}(k b) Y_{\ell " m "}(\hat{b}) T_{\ell "}(k) Y_{\ell " m "}^{*}(\hat{a}) \quad$ (plane wave)

(We will subscript a scattered wave by its origin; for a wave at the detector we replace $\vec{D}$ by $\dot{R}_{\text {.) }}$.

If a spherical wave from a source at the origin is incident on the potential centered at $\vec{a}$, the expansion in spherical harmonics has been derived by Nozawa. ${ }^{14}$ If the spherical wave emanates from the origin, we may expand it around $\vec{a}$ as

$i^{\ell} h_{\ell}(k r) Y_{\ell m}(\hat{r})=\sum_{\ell " m^{n}} G_{\ell m \ell " m^{n}} i^{\ell "} j_{\ell "}(k b) Y_{\ell " m^{\prime \prime}}(\hat{b})$

$G_{\ell m \ell " m "}=\sum_{\ell^{\prime} m^{\prime}} 4 \pi i^{\ell^{\prime}} h_{\ell^{\prime}}(k a) Y_{\ell^{\prime} m^{\prime}}^{*}(\hat{a}) \int Y_{\ell m}(\hat{k}) Y_{\ell^{\prime} m^{\prime}}(\hat{k}) Y_{\ell^{\prime \prime} m^{\prime \prime}}^{*}(\hat{k}) d \hat{k}$

where $\vec{r}=\vec{a}+\vec{b}$. In the mathematical literature, this type of formula is called an addition theorem; we will refer to this equation as Nozawa's origin-shift addition theorem. With this result we can calculate the scattered wave as

$\underset{\vec{a}}{\Psi}(\vec{b})=\sum_{\ell " m^{n}} T_{\ell m}(k) G_{\ell m \ell " m " m^{i}} \ell^{\prime \prime} h_{\ell m}(k b) Y_{\ell " m "}(\hat{b})$

where $G_{\ell m \ell " m \text { " }}$ is $g$ iven above.

To understand and use this formula we must compute the integral of three spherical harmonlcs, called the Gaunt Integral. ${ }^{18}$ This integral can be related to the $3 \mathrm{j}$ vector addition ( $\mathrm{Clebsch-Gordan)}$ symbols ${ }^{19}$ and 
Gaunt has derived an analytic formula to calculate its value. The integral is non-zero only when

$m^{\prime \prime}=m+m^{\prime}, \quad\left|\ell^{\prime \prime}-\ell\right| \leq \ell^{\prime} \leq \ell^{\prime \prime}+\ell \quad \ell^{\prime}+\ell^{\prime \prime}+\ell=$ even

Pendry ${ }^{5}$ gave a computer program to implement Gaunt's formula. Typically a table of these Gaunt integrals is consulted in actual calculations. The complexity of the formula for spherical wave scattering is self-evident. In computer calculations we must recognize that the number of transformation coefficients $G_{\ell m \ell " m}$ is proportional to $\left(\ell_{\max }+1\right)^{4}$ and each coefficient requires the summation of $-2_{\max }$ complex numbers times the Gaunt integral. Since $\ell_{\max }$ is roughly proportional to $k$, multiple scattering calculations already difficult at $k=3 \AA^{-1}$ become prohibitively expensive at $k=12 A^{-1}$. Even this presumes that the Gaunt Integrals are calculated once and stored; for calculations to $600 \mathrm{eV}$ $\left(l_{\max }=19\right),-10^{6}$ integrals are required. For these reasons we must approximate the scattering calculations. 


\section{PHYSICALLY MOTIVATED SMALL ATOM APPROXIMATIONS}

Faced with the intractable spherical wave equations we are led to consider approximate forms. In this section we will examine four approximations, which we will refer to as point scattering, homogeneous wave scattering, plane-wave scattering, and the hybrid, renormalized homogeneous wave method. In the point scattering model, the incident spherical wave is treated exactly, but the potential is taken to have an infinitesimal diameter. This model was introduced by Lee and Pendry ${ }^{8}$ a small atom approximation for EXAFS. The homogeneous wave model allows the potential to have a physical diameter but every incident wave is assumed to have reached its asymptotic limit, $\exp (i k r) / i k r$. The planewave model is the common limit of both the point scattering and homogeneous wave models; it assumes an infinitesimal potential and incldent waves at their asymptotic limit. The renormalized homogeneous wave model combines the mechanics of the point scattering and homogeneous wave methods. Figure 2 compares these approximations graphlcally for an $\ell=7$ spherical wave. We begin by deriving formulae for the scattered waves in each approximation.

A. Point Scattering Model

The motivation for the point scattering model is a practical one: the origin-shift addition theorem for plane waves, eqn. 3 is much simpler than the corresponding formula for spherical waves, eqn. 6 . To repeat the physical approach of Lee and Pendry, ${ }^{8}$ we imagine the outgoing spherical wave meeting a potential with a sufficiently small diameter so 
that we may ignore the curvature of wavefronts coross the potential and the change in wave amplitude and phase along the propagation direction in the region of the potential. In other words, we represent an Incident spherical wave, $i^{l_{h}}{ }_{\ell}(k r) Y_{\ell m}(\hat{r})$, over the infinitesimal point potential by a plane wave with the anplitude and phase of the spherical wave

$1^{\ell} h_{\ell}(k r) Y_{\ell m}(\hat{\imath})=1^{\ell} h_{\ell}(k a) Y_{\ell m}(\hat{a}) e^{i k \hat{a} \cdot(\vec{r}-\vec{a})}$

For a potential of finite diameter, the point-scattering approximate wave will agree with the actual incident wave only at the point $\vec{a}$. As illustrated in figure 2(b), this alignment and the common asymptotic frequency of the exact and approximate waves leads to good agreement between these waves except near the edges of the potential.

We may expand the plane wave with eqn. 3 and use the partial wave prescription to derive the scattered wave as

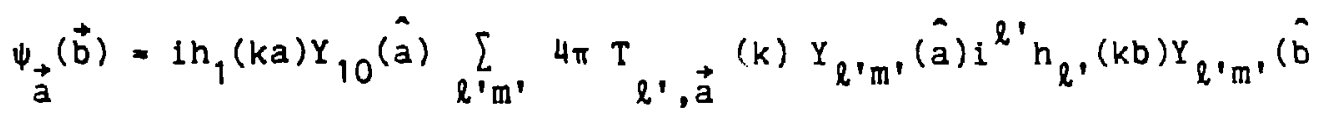

for an incident $\ell=1$ photoelectron wave (eqn. (2)). The same procedure may be applied to the wave emanating from $\vec{a}$ and scattering from a point potential at $\vec{b}$ into the direction of $\vec{c}$ to give a double scattered wave:

$\Psi_{\vec{a} \vec{b}}(\vec{c})=\operatorname{in}_{1}(k a) Y_{10}(\hat{a}) \sum_{\ell^{\prime} m^{\prime}} 4 \pi T l_{\ell^{\prime}, \vec{a}}(k) Y_{\ell^{\prime} m}(\hat{a}) i^{\ell^{\prime}} h_{\ell,}(k b) Y_{\ell, m}(\hat{b})$ 


$$
\sum_{\ell " m "} 4 \pi T \underbrace{}_{\ell ", \vec{b}}(k) Y_{\ell " m "}(\hat{b}) i^{\ell "} n_{\ell "}(k c) Y_{\ell " m "}(\hat{c})
$$

It is useful to separate the spherical hankel function into asymptotic and polynomial parts:

$i_{\ell}^{\ell} h_{\ell}(k r)=\frac{e^{i k r}}{1 k r} d_{\ell}(k r)$

where $d_{\ell}(k r)$ is given by a series ${ }^{20}$ :

$d_{\ell}(k r)=\sum_{p=0}^{\ell} \frac{(\ell+p) !}{p !(\ell-p) !}\left(\frac{1}{2 k r}\right)^{p}$

or, for practical calcuiations, by a recursion formula:

$d_{\ell+1}(k r)=d_{\ell-1}(k r)-\frac{(2 \ell+1)}{1 k r} d_{\ell}(k r) ; \quad d_{0}=1, d_{1}=1+\frac{i}{k r} \cdot(14)$

In the limit that $2 k r \gg \ell(\ell+1), d_{\ell}(k r)=1.0$. We further define a scattering factor

$f_{a b}^{\text {point }}=\frac{1}{i k} \sum_{\ell}(2 \ell+1) T T_{\ell, \vec{a}}(k) d_{\ell}(k b) P_{\ell}\left(\cos \theta_{a b}\right)$

which together with the addition theorem for spherical harmonics:

$4 \pi \sum_{m} Y_{\ell m}(\hat{a}) Y_{\ell m}^{*}(\hat{b})=(2 \ell+1) P_{\ell}\left(\cos \theta_{a b}\right)$ 
allows us to write

$\Psi_{a}(\vec{b})=d_{1}(k a) Y_{10}(\hat{a}) \frac{e^{i k a}}{a} e_{a b}^{p o i n t} \frac{e^{i k b}}{i k b}$

and

$\psi_{a b}(\hat{c})=d_{\ell}(k a) Y_{\ell m}(\hat{a}) \frac{e^{i k a}}{a} f_{a b}^{p o i n t} \frac{e^{i k b}}{b} f_{b c}^{p o i n t} \frac{e^{i k r}}{i k r}$

For a detector at a position $|\vec{R}| \gg \ell_{\max } / k$ the last point scattering factor will converge to the plane wave scattering fdctor of atomic physics:

$\mathrm{f}_{\mathrm{ab}}^{\mathrm{plane}}=\frac{1}{\mathrm{ik}} \sum_{\ell}(2 \ell+1) \mathrm{T}_{\ell, \overrightarrow{\mathrm{a}}}(\mathrm{k}) \mathrm{P}_{\ell}\left(\cos \theta_{\mathrm{ab}}\right)$

and the single-scattered waves calculated at the detector will be

$\Psi_{a}(\vec{R})=d_{1}(k a) Y_{10}(\hat{a}) \frac{e^{i k a}}{a} f_{a R}^{p l a n e} \frac{e^{i k|\vec{R}-\vec{a}|}}{i k R}$

and the double-scattered waves are

$\psi_{a b}(\vec{R})=d_{1}(k a) Y_{10}(\hat{a}) \frac{e^{i k a}}{a} f_{a b}^{p o i n t} \frac{e^{i k b}}{b} f_{b R}^{p l a n e} \frac{e^{i k|\vec{R}-\vec{a}-\vec{b}|}}{i k r}$

B. Homogeneous Wave Model. 
The motivation and formulation of the homogeneous wave scattering model is similar to the point scattering method. The idea is to approximate the incident wave over the region of a finite range potential. If we notice ${ }^{19}$ that a spherical wave reaches to within 10 percent of its asymptotic amplitude when $k r \geq 2 l$, and that the angular distribution of a spherical wave is nearly constant whenever it has a large amplitude, or conversely the angular distribution changes most rapidly near nodes where the amplitude is small, then we are led to approximate the spherical wave by its asymptotic limit, the isotropic spherical wave, $h_{0}(k r)$ :

$i^{\ell} h_{\ell}(k r) Y_{\ell m}(\hat{r})=h_{0}(k r) Y_{\ell m}(\hat{a})$

Since $h_{0}(k r)=\exp (i k r) / i k r$, the homogeneous wave model explicitly incorporates the radial decay characteristic of spherical waves but allows no other variation in wave amplitude over the potential. In particular, the model ignores the amplitude variation of $Y_{\ell m}(\hat{r})$ laterally across the potential, the origin of our name "homogeneous wave" model. Thus the radial form of the incident wave is rather accurately approximated as shown in figure $2(\mathrm{c})$, but the waves are not in phase at the center of the potential. Although not shown by the radial plots in figure 2 , the homogeneous wave model also incorporates the basic curvature characteristic of spherical waves. Thus the phase match between the homogeneous wave and the exact incident wave will appear as in figure $2(c)$ for any radial cut through the wave source, but 
the match between the point scattering wave and the exact wave will be worse than figure $2(b)$ for any cut which does not pass through $\vec{a}$.

The isotropic spherical wave has a simple origin shift addition theorem similar to that of a plane wave:

$h_{0}(k r)=\sum_{\ell "}(2 \ell "+1) 1^{\ell "} j_{\ell "}(k b) i^{\ell "} h_{\ell "}(k a) P_{\ell "}\left(\cos \theta_{a b}\right)$

and we may parallel the development of the point scattering equations with a different scattering factor

$f_{a b}^{00}=\frac{1}{i k} \sum_{\ell "}(2 \ell "+1) T_{\ell ", a}(k) d_{\ell "}(k a) P_{\ell "}\left(\cos \theta_{a b}\right)$,

and arrive at the scattered wave amplitude at the detector in the homogeneous wave approximation

$\Psi_{a}(\vec{R})=Y_{10}(\hat{a}) \frac{e^{i k a}}{a} \rho_{a R}^{00} \frac{e^{1 k|\vec{R}-\vec{a}|}}{1 k R}$

and

$\Psi_{a b}(\vec{R})-\Psi_{10}(\hat{a}) \frac{e^{i k a}}{a} f_{a b}^{00} \frac{e^{i k b}}{b} f_{b R}^{00} \frac{e^{i k|\vec{R}-\vec{a}-\vec{b}|}}{i k R}$.

(The supersoript 00 is retained to parallel the notation of ref. 4 ). McDonnell, et al arrived at similar equations by an entirely different route in their original paper on Auger electron scattering ${ }^{21}$. 
C. Plane Have Model.

Both the point-scattering and homogeneous wave models approach a plane-wave model for large scattering distances $|\vec{a}|$, but the distance at which these models reach the plane-wave limit depends on different parameters. In the point scattering model, the phase and amplitude differences between the spherical wave and a plane wave at the scattering point disappear as the $d_{\ell}(k a)$ approach 1.0 . This requires $2 k a \gg \ell_{\text {in }}\left(\ell_{\text {in }}+1\right)$ where $\ell_{\text {in }}$ is the lincident wave angular momentum. For homogeneous wave scattering we must move the potential far enough away to ignore the variation in $1 / \mathrm{kr}$ across the potential in the direction of propagation; this requires $2 k a \gg l_{\text {out }}\left(l_{\text {out }}+1\right)$ where $l_{\text {out }}$ is the outgoing wave angular momentum. (Note that these limiting criteria only specify the reduction of small atom approximations to the plane-wave limit; they are necessary but not sufficient conditions for plane-wave accuracy compared to the exact curved wave results). In the limit of either model the scattered waves at the detector become

$\Psi_{a}(\vec{R})=Y_{10}(\hat{a}) \frac{e^{i k a}}{a} f_{a R}^{p l a n e} \frac{e^{i k|\vec{R}-\vec{a}|}}{i k R}$

and

$\Psi_{a b}(\vec{R})=Y_{10}(\hat{a}) \frac{e^{i k a}}{a} f_{a b}^{p l a n e} \frac{e^{i k b}}{b} f_{b R}^{p l a n e} \frac{e^{i k|\vec{R}-\vec{a}-\vec{b}|}}{i k R}$ 
It is this "plane-wave" form of the scattering equations that leads to the simple single scattering formulas for EXAFS ${ }^{8}$ and ARPEFS. 15

Neither the point scattering nor homogeneous wave models have persuasive advantages over the plane-wave limit in general scattering problems. Point scattering models the incident wave well at one point, without regard for the size of the potential. For point scattering to apply, we must be able to ignore the variation in $1 / \mathrm{kr}$ across the potential; from the homogeneous wave equations we can see this requires

$$
2 k a \gg l_{\text {out }}\left(l_{\text {out }}+1\right) \text {. }
$$

Whenever this requirement is fulfilled, we have $2 k a \gg \ell_{\text {in }}\left(l_{\text {in }}+1\right)$ for all $\ell_{\text {in }}$ - $\ell_{\text {out }}$ and the plane-wave 1 imit will be reached by the incident wave. In other words, only if we scatter high angular momentum waves off a short range potential where $\ell_{\text {in }} \gg \ell_{\text {out }}$ will point scattering significantly improve on the plane wave model. Roughly the converse is true for the homogeneous wave formulas. The homogeneous wave method considers the size of the potential, but approximates the incident wave. To ignore the difference in phase between the incident spherical wave and the isotropic homogeneous wave, $h_{0}(k r)$, we must have $\ell_{\text {in }}\left(\ell_{\text {in }}+1\right)<<$ 2ka. Whenever this requirement is fulfilled, we may ignore wave-front curvature for all $\ell_{\text {out }} \leq \ell_{\text {in }}$. Thus the homogeneous wave model will only improve on the plane-wave model for scattering low angular momentum waves from potentials with large effective radii, potentials which give rise to high angular momentum waves.

Despite these restrictions there are important problems to which these approximations apply. For the EXAFS single-scattering geometry, a 
complete range of angular momentum waves are backscattered to the central atom, but only low angular momenta can couple through the dipole matrix element to the initial state; waves striking the central atom have $\ell_{\text {in }} \gg l_{\text {out }}$. For the ARPEFS single-scattering problem, dipole excitation gives only low angular momentum waves for scattering and, since the high angular momentum waves only appear asymptotically, we have some cases of $\ell_{\text {out }} \geq \ell_{\text {in }}$ : On the whole we might rank the homogeneous wave model ahead of the point scattering or plane-wave models more general scattering problems: low angular momentum partial waves carry much more weight when the scattered wave is constructed. We will also show in section IV that the homogeneous wave model is the zero order Tayior series term.

Our distinction between incident and outgoing angular momenta highlights the distinction - commonly overlooked - between the asymptotic limit of spherical Hankel functions and the plane wave limit of spherical waves. The asymptotic limit of $1^{\ell} h_{\ell}(k r)$ is $h_{0}(k r)=\exp (1 k r) / 1 k r$ and we may Invoke this 11 mit whenever $2 k r \gg l(l+1)$. Even if the asymptotic limit is justified, the plane-wave limit may still fail to apply: the variation in $(1 / \mathrm{kr})$ across the potential may be signiflcant if the potential has a large diameter. Conversely, the variation in (1/kr) may be neglected for a small diameter potential, but, if the incident angular momentum is high, we are not in the asymptotic limit of the spherical Hankel function. The plane-wave limit incorporates two approximations: the asymptotic limit and a negligible diameter potential. 
D. Hybrid Model.

our contention that the point scattering and homogeneous wave models have nearly opposite ranges of use would suggest a hybrid "renormalized" homogeneous wave method in which the phase and amplitude of the incident spherical wave is attached to the isotropic wave before scattering. Thus over the region of the potential we would represent the incident wave by

$$
i^{\ell} h_{\ell}(k r) Y_{\ell m}(\hat{r})=d_{\ell}(k a) Y_{\ell m}(\hat{a}) h_{0}(k r) .
$$

This approximation would agree with the incident wave in radial form (1/kr decay) and in phase and amplitude at $\vec{a}$ as shown in figure $2(d)$, and the phase agreement would extend to all points with $|\vec{r}|=|\vec{a}|$. While such a small atom approximation would gi ve good results for the EXAFS and ARPEFS single scattering cases ${ }^{22}$, we have no guarantee of success in multiple scattering problems: the criteria for the application of the small atom models we have examined thus far are necessary but not sufficient conditions for arcuracy. The most serious limitation shared by these small atom approximations lies in directions not graphed in figure 2: none of the models described so $f$ ar account for variation in wave amplitude across the potential due to the angular dependence of the spherical wave.

Rather than explore further the range of validity for these small atom approximations, we turn instead to the development of a new fifth approximation which allows steady improvement toward the exact curvedwave result. The development of this Taylor series, small atom approximation will comprise the following section; as a prelude we close 
this section with two topics related to the plane-wave model. The first is simply the mathematical reduction of the exact origin-shift addition theorem, eqn. 6, to the plane wave limit; the required approximation bears upon our discussion above. Second, we attempt to understand the formally disconcerting but empirically well-founded success of the plane wave model, by listing several contributions to its usefulness.

E. Formal and Practical Plane-Wave Limits.

We may arrive at the plane-wave limit by replacing the spherical Hankel functions in Nozawa's origin-shift addition theorem by their asymptotic forms. We may then move the intermediate sum in equation 6 inside the Gaunt integral and, using the closure sum for spherical harmonics, perform the angle integration to conclude that

$G_{\ell m \ell m^{\prime \prime}}(k \vec{a})-4 \pi \frac{e^{i k a}}{i k a} Y_{\ell m}(\hat{a}) Y_{\ell " m^{\prime \prime}}^{*}(\hat{a})$

The addition theorem for spherical harmonics (eqn. 16) and the partial wave method then $\mathrm{gi}$ ves eqn. 27 .

We may give the criterion for applying this approximation to Nozawa's origin-shift addition theorem as $\ell^{\prime}\left(\ell^{\prime}+1\right) \ll 2 k$ wher? $\ell^{\prime}$ is ihe angular momentum of the intermediate sum in equation 6 . The maximum intermediate angular momentum is restricted. by the non-zero Gaunt Integrals, eqn. 8 , to be $\ell^{\prime}=\ell^{\prime \prime}+\ell$ or $\ell^{\prime}=\ell_{\text {in }}+\ell_{\text {out }}$ using our notation from above. This sufficient condition for the plane-wave lidit is much more restrictive than either conditions for the small atom 
approximations and would indicate that plane-wave formulas would be adequate only for low angular momenta scattering from very short range potentials. We turn then to list some contribution to the success of the plane wave limit when $2 k a$ is not much greater than $\left(l_{\text {in }}+\ell_{\text {out }}\right)^{2}$.

Let us now try to understand the success of the plane wave method despite the evident theoretical problem. There are five important contributions:

i) The phase difference between the scattered wave and the unscattered wave is dominated by their different origins. Thus if we measure the phase difference between a backscattered wave and an unscattered wave, the phase calculated in the plane wave approximation need only be the same magnitude as that given by the spherical wave formula. ${ }^{2}$

11) Spherical Hankel functions reach ${ }^{19}$ to within 10 percent of their large $\mathrm{kr}$ limit when $\mathrm{kr}-2 l_{\max }$.

1ii) At large $k$, the large number of contributing partial waves reduces the fractional error made in approximate treatment of the highest $\ell$ waves. In other words, the low $\ell$ waves have reached their asymptotic limit and the high $\ell$ waves become outnumbered.

iv) It has been discovered empirically from EXAFS analysis that the phase difference in the plane wave limit for backscattering may be reliably corrected by slight shifts in the scattering energy. ${ }^{2}$ While discomforting from a 
theoretical viewpoint, the scattering energy is not measured in the $x$-ray absorption experiment anyway. 23

v) Finally, as discussed in reference 4, curved wave corrections are much smaller for backscattering due to cancellation of successive partial waves, or equivalently, as illustrated in figure 3, a smaller region of the potential contributes to backscattering.

Taken together, these 1 deas begin to explain the substantial success of the plane wave model. To make further progress in understanding the electron scattering or to enable efficient, accurate numerical calculation, especially for forward scattering directions, we must seek some approximation between the plane and full spherical wave formulas. 


\section{TS-MQNE APPROXIMATION}

To derive an approximate method beyond those discussed in the previous section we return to the exact Gaunt integral summation formula, eqn. 6, and ask how the known characteristics of the scattering problem might be used to eliminate physically unnecessary aspects of the mathematically exact origin-shift addition theorem. Any approximation scheme must recognize that we require an expansion with a particular form: it must be an angular momentum expansion about the potential center. Our solution is to expand the Fourier transform of the spherical wave in a Taylor series about the direction of the origin-shift vector, $\vec{a}$, translate the individual terms of the expansion, and obtain an angular momentum series when each term is subjected to the inverse Fourier transform. The result is a finite series capable of performing every duty of an origin-shift addition theorem, so we have reported its development separately ${ }^{13}$. The formula is

$$
\begin{aligned}
i^{\ell} h_{\ell}(k r) Y_{\ell m}(\hat{r})= & \sum_{q=-\ell}^{\ell} N_{\ell q} R_{q m}^{(\ell)}\left(0, \theta_{\varepsilon a} \pi^{--\phi_{x \in a}}\right) \sum_{p=0}^{\ell-|q|} c_{p q}^{\ell} \\
& \sum_{\ell "=q}^{\infty} 4 \pi i^{\ell "} j_{\ell "}\left(k r^{\prime}\right) Y_{\ell " q}\left(\hat{r}^{\prime}\right) N_{\ell " q} h_{0}^{(k a) H_{\ell "}^{p q}(k a)}
\end{aligned}
$$

where $N_{\ell q}$ is a normalizing coefficient for spherical harmonics, $R(\ell)$ is a rotation matrix element ${ }^{19}, C_{p q}^{\ell}$ contains factorials from the expansion of Legendre polynomials, and $\mathrm{H}_{\ell}^{\mathrm{pq}}(\mathrm{ka})$ may be expressed as an integral or 
various series. The precise definition of the factors is given in ref. 13. This reference also describes an alternative view of the expansion in which the variable $q$ is a magnetic quantum number in a coordinate system rotated to align the scattering and quantization axes. We will explore this magnetic quantum number expansion (MQNE) viewpoint in section $V$, but we will use the acronym now to refer to eqn. 31 . To calculate the wavefield due to scattering of the direct wave, eqn. 2, from a potential at a polnt $\vec{a}$, we first expand the direct wave about $\vec{a}$ using eqn. 31 . For $\ell=1$, a first order Taylor expansion is exact:

$$
\begin{aligned}
& \operatorname{in}_{\uparrow}(k r) Y_{10}(\hat{r})=N_{10} R_{00}^{\ell}\left(0, \theta_{\varepsilon a},{ }^{--\Phi_{x \varepsilon a}}\right) \sum_{\ell} i^{\ell} j_{\ell}(k b) Y_{\ell 0}(\hat{b}) N_{\ell 0} 4 \pi\left[H_{\ell}^{00}+H_{\ell}^{10}\right] \\
& +N_{11} R_{10}^{\ell}\left(0, \theta_{\varepsilon a}, \pi-\phi_{x \in a}\right) \sum_{l} i^{\ell} j_{\ell}(k b) Y_{\ell 1}(\hat{b}) N_{\ell-1} 4 \pi H_{l}^{01} \\
& +N_{1-1} R_{-10}^{\ell}\left(0, \theta_{\varepsilon a,} \pi-\phi_{x \varepsilon a}\right) \sum_{\ell} i^{\ell} j_{\ell}(k b) Y_{\ell-1}(\hat{b}) N_{\ell 1} 4 \pi H_{l}^{01}
\end{aligned}
$$

The rotation matrix elements are particularly simple when one of the subscripts is zero ${ }^{19}$ :

$$
R_{m 0}^{\ell}(\alpha, \beta, \gamma)=\left(\frac{4 \pi}{2 \ell+1}\right)^{1 / 2} Y_{\ell m}^{*}(\beta, \alpha)
$$

Note all the magnetic sublevels here refer to the $\hat{a}$ axis (see mef 13 , App. Bi. For $\ell=1$, 
$N_{10} R_{00}^{1}\left(0, \theta_{\varepsilon a}, \pi-\phi_{x \varepsilon a}\right)=\left(\frac{3}{4 \pi}\right)^{1 / 2} \cos \theta_{\varepsilon a}$

and

$N_{11} R_{10}^{1}\left(0, \theta_{\varepsilon a},{ }^{-\phi_{x \in a}}\right)=N_{1-1} R_{-10}^{1}\left(0, \theta_{\varepsilon a}, \pi^{-\phi_{x \varepsilon a}}\right)=\frac{1}{2}\left(\frac{3}{4 \pi}\right)^{1 / 2} \sin \theta_{\varepsilon a}$

To calculate the scattered wave emanating from the potential at center $\hat{a}$, we replace $i^{\ell} j_{\ell}(k b)$ by $T_{\ell}(k) i^{l} h_{\ell}(k b)$ in the origin-shift formula. This single-scattered wave can then propagate to our detector or scatter again. We flrst consider the single-scattered wave at our detector.

For a detector at a position $|\overrightarrow{\mathrm{A}}| \gg|\vec{a}|$, the outgoing, scattered partial waves have all reached their asymptotic limit. As will be more apparent for the multiple scattering equations, it is convenient to define a new scattering factor

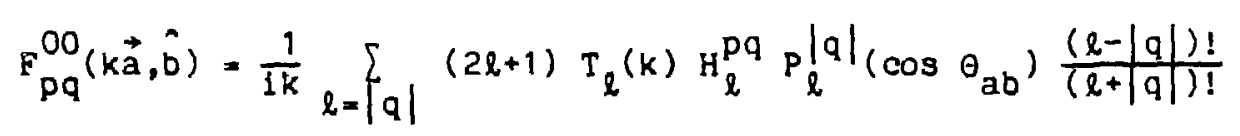

so that the single-scattered wave may be written

$$
\begin{aligned}
\Psi_{a}(\vec{R})= & \left(\frac{3}{4 \pi}\right)^{1 / 2} \frac{e^{i k R}}{1 k R} \frac{e^{1 k a\left(1-\cos \theta_{a R}\right)}}{a} \\
& \left\{\cos \theta_{\varepsilon a}\left[F_{00}^{00}(k \vec{a}, \vec{R})+F_{10}^{00}(k \vec{a}, \hat{R})\right]+\sin \theta_{\varepsilon a} \cos \phi_{\varepsilon a R} F_{01}^{00}(k \vec{a}, \hat{R})\right\}
\end{aligned}
$$

Reference 4 presents this same result in a different notation; Appendix A provides the connection. 
Let us now return to the single-scattered wavefield near center $\vec{a}$ and tackle the multiple scattering case. The (exact) Taylor series origin shift, equation 32 , and the partial wave phase shifts give the single-scattered wave in the near field region as

$$
\begin{aligned}
& \psi_{a}(\vec{b})=\frac{e^{i k a}}{a} \sum_{q=-1}^{1} N_{1 q} R_{q 0}^{1}\left(0, \theta_{\varepsilon a}, \pi-\phi_{x \in a}\right) \sum_{p=0}^{1-|q|} C_{p q}^{1} \\
& \frac{1}{i k} \sum_{\ell=|q|} 4 \pi T_{\ell, \vec{a}}(k) H_{\ell}^{p q_{(k a)}} N_{\ell q} i^{\ell} h_{\ell}(k b) Y_{\ell q}(\hat{b})
\end{aligned}
$$

The dependence on $\vec{b}$ in each term of this expansion is that of a spherical wave, $i^{\ell} h_{\ell}(k b) Y_{\ell q}(\hat{b})$, and as this wave encounters another potential at position $\vec{b}$, we can apply the MQNE formula and partial wave phase shifts again to calculate the double scattered wave. If we call $\psi_{a b}^{\ell q}(\vec{R})$ the wave generated when the $(l, q)$ spherical wave from $\vec{a}$ scatters from $\vec{b}$ and is detected at $\vec{R}$, then we have

$$
\begin{gathered}
\psi_{a b}^{\ell q}(\vec{R})=\sum_{q^{\prime}=-\ell}^{\ell} N_{\ell q^{2} q^{R^{\prime}} q^{\ell}\left(0, \theta_{a b^{\prime}}, \pi-\phi_{\varepsilon a b}\right)} \sum_{p^{\prime}=0}^{\ell-\left|q^{\prime}\right|} c_{p^{\prime} q^{\prime}}^{\ell} e^{i q^{\prime} \phi_{a b R}} \frac{e^{i k b}}{b} \frac{e^{i k|\vec{R}-\vec{a}-\vec{b}|}}{i k|\vec{R}-\vec{a}-\vec{b}|} \\
\left.\qquad \frac{1}{1 k} \sum_{\ell^{\prime}=q^{\prime}}^{\ell}\left(2 \ell^{\prime}+1\right) T_{\ell^{\prime}, b^{\prime}}(k) H_{\ell^{\prime}}^{p^{\prime} q^{\prime}}(k b) P_{\ell^{\prime}}^{\left|q^{\prime}\right|}\left(\cos \theta_{b R^{\prime}}\right) \frac{\left(\ell^{\prime}-\left|q^{\prime}\right|\right) !}{\left(\ell^{\prime}+\left|q^{\prime}\right|\right) !}\right\}
\end{gathered}
$$

We have separated the partial waves which reach the detector into azimuthal, polar, and radial components so that we may recognize the 
factor in braces as $F_{p^{\prime}}^{00} q^{\prime}$, defined above. Summing over all of the $(l, q)$ partial waves gives the complete double scattered wave at the detector as

$$
\begin{aligned}
& W_{a b}(\vec{R})=\frac{e^{i k a}}{a} \frac{e^{i k b}}{b} \frac{e^{i k|\vec{R}-\vec{a}-\vec{b}|}}{i k|\vec{R}-\vec{a}-\vec{b}|} \sum_{q=-1}^{1} N_{q} q_{q 0}^{1}\left(0, \theta_{\varepsilon a}, \pi-\phi_{x \in a}\right) \sum_{p=0}^{1-|q|} C_{p q}^{1} \\
& \sum_{q^{\prime}=-\tau}^{\tau} \sum_{p^{\prime}=0}^{\tau-\left|q^{\prime}\right|} e^{i q^{\prime} \phi_{a b R}} F_{p^{\prime} q^{\prime}}^{00}(k \vec{b}, \hat{R})
\end{aligned}
$$

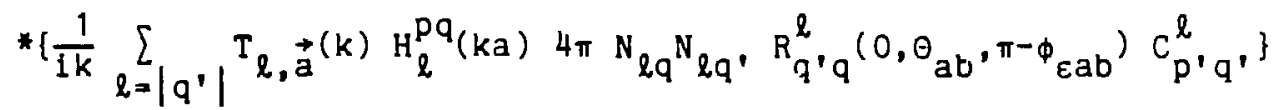

We have reordered the sums on $\ell, p^{\prime}$, and $q^{\prime}$ to isolate the factor in braces. In that process we introduced the Taylor series order, $\tau$, as the limit for the sums on $q^{\prime}$ and $p^{\prime}$. The factor in braces gives the amplitude for waves of order $p q$ to scatter off the potential at $\vec{a}$ and give waves of order $p^{\prime} q^{\prime}$ in the direction of $\vec{b}$. Our next step is to define most of the factor in braces as a Taylor serles spherical wave scattering factor, $F_{p q}^{p^{\prime} q^{\prime}}$, where $F_{p q}^{00}$ defined above is a special case.

To this end we break up the rotation matrix into polar and azimuthal parts as given by Messiah ${ }^{19}$ :

$R_{q^{\prime} q^{\ell}}^{\ell}(\alpha \beta Y)=e^{-i \alpha q^{\prime}} r_{q^{\prime} q^{(\ell)}}^{(\ell)} e^{-i \gamma q}$

For our Euler angles we get

$R_{q^{\prime} q^{\ell}}^{\ell}\left(0, \theta_{a b}, \pi-\phi_{\varepsilon a b}\right)=(-1)^{q} e^{1 q \phi_{\varepsilon a b}} r_{q q^{\prime} q}^{(\ell)}\left(\theta_{\varepsilon a}\right)$ 
Edmonds ${ }^{24}$ gives a formula for $r_{q^{\prime} q}^{l}\left(-\theta_{\varepsilon a}\right)$ : his rotation is the inverse of Messiah's. A symmetry in the rotation matrix allows his formula to be used by switching indices:

$$
\begin{aligned}
& \Gamma_{q^{\prime} q}^{(l)}\left(\theta_{\varepsilon a}\right)=\left[\frac{(l+q) !(l-q) !}{\left(l+q^{\prime}\right) !\left(l-q^{\prime}\right) !}\right]^{1 / 2} \\
& \qquad \cos ^{q+q^{\prime}}\left(\frac{{ }_{\varepsilon a}^{\theta_{\varepsilon a}}}{2}\right) \sin ^{q-q^{\prime}}\left(\frac{\left.{ }_{\varepsilon a}\right)}{2} P_{\ell-q}^{\left(q-q^{\prime}, q+q^{\prime}\right)}\left(\cos \theta_{\varepsilon a}\right)\right.
\end{aligned}
$$

where $P_{\ell}^{(\alpha, \beta)}(\cos \theta)$ is the Jacobi polynomial. If $q^{\prime}>$ q we use: ${ }^{19}$

$$
r_{q^{\prime} q}^{(l)}\left(\theta_{E a}\right)=(-1)^{q-q^{\prime}} r_{q q^{\prime}}^{(l)}\left(\theta_{\varepsilon a}\right)
$$

to avoid negative quantum numbers in the Jacobi polynomial; similarly if $q+q^{\prime}<0$ we use

$$
r_{q^{\prime} q^{\left(\theta_{\varepsilon a}\right.}}^{(l)}=(-1)^{q-q^{\prime}} r_{-q-q^{\prime}}^{(l)}\left(\theta_{\varepsilon a}\right)
$$

for the same purpose.

Removing the azimuthal dependence from the factor in braces in equation (40) leads to our scattering factor,

$$
F_{p q}^{p^{\prime} q^{\prime}}(k \vec{a}, \hat{b})=\frac{1}{i k} \underset{\ell=|\vec{q}|_{>}(2 \ell+1) T}{\ell, \vec{a}}\left(k j H_{\ell}^{p q}(k a) \frac{(\ell-|q|,) !}{\left(\ell+|q|_{<}\right) !} C_{p^{\prime} q^{\prime}}^{\ell}\right.
$$




$$
\left(\cos \frac{\theta_{a b}}{2}\right)^{\left|q+q^{\prime}\right|}\left(\sin \frac{\theta_{a b}}{2}\right)^{\left|q-q^{\prime}\right|} \underset{p l-|q|}{\left(\left|q-q^{\prime}\right| \cdot\left|q+q^{\prime}\right|\right)}\left(\cos \theta_{a b}\right)
$$

We have inserted the value of $N_{\ell q} N_{\ell q}$ ' and mean for $|q|,\left(|q|_{<}\right)$to be the greater (lesser) of $|q|$ and $\left|q^{\prime}\right|$ : We may similarly define the overlap of the direct wave with angular momenta eigenfunctions in the direction of $\hat{a}$ by

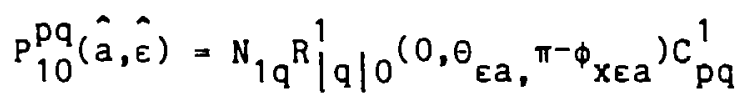

We also incorporate the signs from $\mathrm{N}_{\ell q}$, $\mathrm{N}_{\ell q}{ }$, the factor of $\exp$ (iqm), and the symmetry relations for the rotation matrices into:

$S_{q}^{q}=(-1)^{q} \quad|q-q \cdot|+|q|-|q \cdot|$

as shown in Appendix $B$.

The single-scattered wave at the detector then becomes

$$
\begin{aligned}
& \psi_{a}(R)=\frac{e^{i k a}}{a} \frac{e^{1 k|\vec{R}-\vec{a}|}}{1 k R} \\
& \sum_{q=-1}^{1} \sum_{p=0}^{1-|q|} F_{p q}^{00}(k a, \hat{R}) e^{i q \phi} \varepsilon a R P_{10}^{p q}(\hat{a}, \hat{\varepsilon})
\end{aligned}
$$

The double-scattered wave is: 


$$
\begin{aligned}
& \psi_{a b}(R)=\frac{e^{i k a}}{a} \frac{e^{i k b}}{b} \frac{e^{i k|\vec{R}-\vec{a}-\vec{b}|}}{1 k R} \sum_{q^{\prime}=-\tau}^{\tau} \sum_{p^{\prime}=0}^{\tau-|q|} F_{p^{\prime} q^{\prime}}^{00}(k \vec{b}, \hat{R}) s_{q^{\prime}}^{0} e^{i q^{\prime} \phi_{a b R}} \\
& \sum_{q=-1}^{1} \sum_{p=0}^{1-|q|} F_{p q}^{p^{\prime} q^{\prime}}(k \vec{a}, \hat{b}) s_{q}^{q^{\prime}} e^{1 q \phi} \varepsilon a b p_{10}^{p q}(\hat{a}, \hat{\varepsilon})
\end{aligned}
$$

and the triple-scattered wave may be written by inspection. These expressions constitute our fifth small atom approximation for the multiple scattering of photoelectrons. 


\section{v. THE TAYLOR SERIES SCATTERING FACTORS}

Convergence of the approximate curved-wave scattering formulae derived in the previous section requires the scattering factors $F_{p q}^{p^{\prime} q^{\prime}}(k \vec{a}, b)$ to decrease rapidly as the Taylor indices $p, q, p^{\prime}, q^{\prime}$ increase. We devote this section to a discussion of these factors. The success of plane-wave scattering models does not follow from simple convergence arguments, leading us to suspect any purely formal criterla for the Taylor series approach. Rather than pursue a general account of the convergence we will discuss some of the properties of the scattering factors using $\mathrm{Ni}$ atom scattering as an illustrative example.

The scattering factor depends on the four indices $p, q, p^{\prime}$, and $q^{\prime}$, on the wavenumber-distance product $k a$, on the scattering angle between $\hat{a}$ and $\hat{b}, \theta_{a b}$, and on the potential through the scattering phase shifts $\mathrm{T}_{\ell, a}(\mathrm{k})$. We refer to the superscript indices as outgoing wave indices and to the subscript indices as incoming wave indices. The single zeroorder scattering factor, $F_{00}^{00}$, has been discussed in reference 4 (see Appendix A.); its dependence on angle and energy is qualitatively similar to the plane-wave scattering factor. As we consider larger values of the indices we find that scattering factors with non-zero outgoing wave superscripts are large while scattering factors with nonzero incoming wave subscripts are correspondingly small. When the scattered waves are formed by combining these scattering factors, large outgoing wave indices are always paired with identical incoming wave

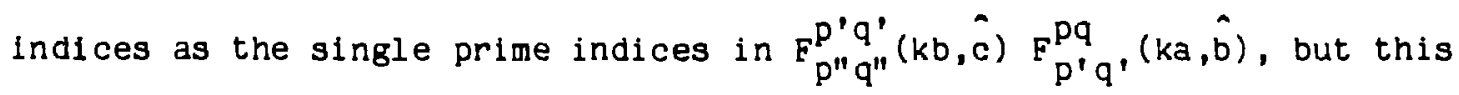
behavior makes convergence difficult to discuss. Thus we will rescale 
the scattering factors so that they may stand alone. Our development of this rescaled factor will also serve as our introduction to the nature of the scattering factors.

Our goal for the rescaled scattering factor is to isolate the dominate character of $\mathrm{F}_{\mathrm{pq}}^{\mathrm{P}^{\prime} \mathrm{q}^{\prime}}$ which allows it to converge as $\mathrm{p}$ and $\mathrm{q}$ increase and to split this character between the incoming and outgoing waves. The incoming wave indices $p$ and $q$ appear in the integral $\mathrm{H}_{\ell}^{\mathrm{PQ}}(k a)$; the $\mathrm{q}$ index also appears in the angle functions, which we discuss first.

The angle dependent terms in the scattering factor are:

$A_{q q},\left(\theta_{a b}\right)=$

$$
\left[\cos \frac{\theta_{a b}}{2}\right]^{\left|q^{\prime}+q^{\prime}\right|}\left[\sin \frac{\theta_{a b}}{2}\right]^{\left|q^{\prime}-q^{\prime}\right|}{ }_{P_{\ell-|q|}}^{\left(\left|q-q^{\prime}\right|, \mid q^{\mid}\left(\cos \theta_{a b} \mid\right)\right.} .
$$

For the purpose of rescaling the scattering factor we may ignore these angle terms: their product always has a magnitude less than, but on the same scale as 1.0. In passing, we note that the angle dependence requires that

i) only factors with $q=q$ ' contribute to forward $\left(\theta_{a b}=0\right)$ scattering,

ii) only factors with $q=-q^{\prime}$ contribute to backscattering $\left(\theta_{a b}=\pi\right)$,

iii) the angular factors do not change if both $q$ and $q^{\prime}$ are simultaneously negated or if $q$ and $q^{\prime}$ are switched, 
iv) the angular factors alternate sign with increasing $\ell$ for $\theta>\pi / 2$.

Item $i(i)$ is a consequence of the symmetry relations of the rotation matrix elements and, coupled with the observation that all the remaining dependence on $q$ and $q^{\prime}$ in the scattering factor uses only their magnitudes, we have this important relation:

$F_{p q}^{p^{\prime} q^{\prime}}(k \vec{a}, \vec{b})=r_{p-q}^{p^{\prime}-q^{\prime}}(k \vec{a}, \hat{b})$

With no strong dependence on $q$ in the angular pactors, we must turn to $H_{\ell}^{p q(k a)}$ for our rescaling relation. If we use the explicit formula from appendix $C$, ref. 13, we may write:

$$
\begin{aligned}
& F_{p q}^{p^{\prime}}\left(q_{k}^{\prime} \vec{a}, \hat{b}\right)=\frac{1}{1 k} \frac{(-1)^{p}}{(1 k a)^{p+q}} \frac{p !}{q !} \sum_{s=0}^{p} \frac{(p+q-s) !}{s !(p-s) !}(k a)^{s} \sum_{\ell=|q|_{>}}(2 \ell+1) T_{\ell, a}(k) \frac{\partial^{s} d_{\ell}(k a)}{\partial(k a)^{s}} \\
& \frac{(\ell+|q|) !}{(\ell-|q|) !} \frac{(\ell-|q|,) !\left(\ell+\left|q^{\prime}\right|+p^{\prime}\right) !}{\left(\ell+|q|_{<}\right) !\left(\ell-\left|q^{\prime}\right|-p^{\prime}\right) !} \frac{1}{p^{\prime} !(2|q|+2 p) ! !{ }_{q q}^{\prime}\left(\theta_{a b}\right)}
\end{aligned}
$$

The leading term and the factorial terms containing $\ell$ in this equation reiterate an earlier theme from our discussion of the convergence of the small atom approximations in section III: the size of these scattering factors is determined by $\ell(\ell+1) / \mathrm{ka}$. The product of factorials depending on $\ell$ in this form of the scattering factor may be expanded into a 
polynomlal in $\ell^{2}$ whose highest power is $\left.p^{\prime}+i\left|q^{\prime}\right|+|q|\right) / 2$. From these results we propose a rescaled scattering factor according to

$\bar{F}_{p q}^{p^{\prime} q^{\prime}(k \vec{a}, \hat{b})}=\frac{\left(k r_{0}\right)^{p+|q| / 2}}{\left(k r_{0}\right)^{p^{\prime}+\mid q^{\prime} / / 2}} F_{p q}^{p^{\prime} q^{\prime}(k \vec{a}, \hat{b})}$

Empirically we find that all the rescaled factors for nearest neighbor scattering are of a similar size. (We must also apply this rescaling to the photoemission factors, $P_{10}^{p^{\prime}} q^{\prime}$ to insure that the scaling always cancels when the wavefunctions are constructed.)

If we estimate the scattering radius by $\mid f(\theta, k) i / 2$, our rescaled scattering factor is roughly proportional as

$\bar{F}_{p q}^{p^{\prime}} q_{(k \vec{a}, \hat{b})} \propto\left(\frac{r_{0}}{a}\right)^{p+q}\left(\frac{|f(\theta, k)|}{2 r_{0}}\right)^{p^{\prime}+\left(q^{\prime}+q\right) / 2}\left(\frac{k|f(\theta, k)|}{2}\right)^{p^{\prime}+\left(q^{\prime}+q\right) / 2}$

The first term in this expression contains increasing powers of $\left(r_{0} / a\right)$, the angle subtended by the radius of the potential at a distance $|\vec{a}|$ from the wave source. Thus if we compare scattering factors at a bond length of $|\vec{a}|$ and $2|\vec{a}|$, we will find that first order terms are half as large, the second order terms are one-iourth as $l$ arge and so on. The second term may be interpreted as correcting the scattering potential radius to account for the scattering angle differences: forward scattering angles will have $[|f(\theta, k)| / 2]=r_{0}$ while backscattering only uses the central region of the potential, $[|f(\theta, k)| / 2]<<r_{0}$. Hence the 
convergence in backscattering is much more rapid. Finally, the last term reminds us that the convergence does not improve with energy; it may indeed worsen.

Representative examples of second order scattering factors for nearest neighbor $\mathrm{NI}$ atom scattering are illustrated in figures 4, 5, and 6. The more rapid convergence in backscattering is evident by the small values of the higher order scattering factors for angles $>90^{\circ}$, and a first order Taylor series appears adequate for backscattering. Forward angles may require higher than second order. 
VI. DISCUSSION

In the previous section we have shown that Taylor series terms of order $\tau$ fall off as $(1 / k a)^{\tau}$ where a is the bond length between scattering atoms, and we suggested that the size of the Taylor terms is governed by $\left(r_{0} / a\right)^{\top}$.

In this section we will

i) discuss the zero order term which survives at larger bond lengths,

ii) give an example of the convergence of the series for a particular nearest neighbor scattering,

ii1) describe the magnetic quantum number expansion view of the physical nature of the Taylor series small atom approximation, and

iv) describe how this alternative view predicts rapid convergence of the Taylor expansion for multiple scattering ARPEFS problems.

We begin with the zero-order Taylor series term. With $\tau=0$, the scattering equations from section IV contain only one scattering factor for each event:

$$
F_{00}^{00}(k \hat{a}, \hat{b})=\frac{1}{i k} \sum_{\ell=0}(2 \ell+1) T_{\ell}(k) d_{\ell}(k a) P_{\ell}\left(\cos \theta_{a b}\right) .
$$

This is exactly equal to the homogeneous wave scattering factor $f_{a b}^{00}$ : the homogeneous wave model introduced in section III is the zero-order Taylor series term. This means that the zero-order term represents the scattering of spherical Hankel functions at their asymptotic limit, but 
it contains the $1 / \mathrm{kr}$ variation of $h_{0}(\mathrm{kr})$ radially along the potential. Note that the zero-order term is not the plane-wave model, but, as discussed in ref 4 , the difference between the plane-wave factor $\mathrm{f}_{\mathrm{ab}}^{\mathrm{plane}}$ and $\mathrm{f}_{\mathrm{ab}}^{00}$ is small for backscattering angles. Coupled with rapid convergence of the Taylor series for backscattering angles, we conclude that the plane-wave approximation may be adequate for many backscattering problems.

The same may not be said for scattering angles closer to zero. To give some feel for the size of the corrections for forward scattering, we have calculated the ARPEFS oscillations for a particularly important geometry. We have selected our problem from the experimental study of ref 1: we consider $S(1 s)$ photoabsorption from $c(2 \times 2) S / N i(100)$ with both the emission and polarization vectors along the [110] crystallographic direction. We concentrate on only two scattering events, single scattering from the $\mathrm{Ni}$ atom directly behind the $\mathrm{S}$ photoemitter and forward scattering of this backscattered wave through the $\mathrm{S}$ emitter. The path-length difference between these scattered waves and the direct wave are nearly equal at $-4.4 \mathrm{~A}$, corresponding to the dominant frequency in the experimental measurements. The backscattering angle is $173^{\circ}$ while the forward angle is $7^{\circ}$.

The results of these calculations are displayed in Figures 7 and 8 as

$$
x(k)=\frac{\psi_{s}^{*} \psi_{s}-\psi_{0}^{*} \psi_{0}}{\psi_{0}^{*} \psi_{0}}
$$

where 


$$
\psi_{s}=\psi_{0}+\psi_{a}+\psi_{a b}
$$

for $\vec{a}$ running from the $S$ emitter to the $N i$ scattering atom and $\vec{b}=-\vec{a}$. Figure 7 compares plane-wave calculations with the "exact" spherical wave calculation based on the Gaunt integral summation. The osclllations in the plane wave case are much larger even though the plane wave model slightly underestimates the forward scattering amplitude. This means that the most of the discrepancy is the phase error made in the plane-wave forward scattering. The forward scattering phase shift estimated by the plane-wave model is too small. Thus the single scattered and double scattered waves are nearly in phase and their sum has an amplitude 170 of the single scattering amplitude. With the correct forward-scattering phase-shift, the double-scattered wave is $-\pi / 2$ out of phase with the single scattered wave and the sum has a more modest amplitude.

Figure 8 illustrates the Taylor series model results. We are able to display only the zero-order result on this scale: the first-order Taylor series cannot be distinguished from the exact calculation. Thus at least for this important scattering geometry, the Taylor series is converged at first order. Our alternative view of the Taylor model which we develop next will help to understand this remarkable convergence and will lead into our discussion of more general scattering geometries.

Before leaving figure 8 we note that the phase of the zero order (homogeneous wave) result is accurate while the amplitude is too large. This means that important differences between the asymptotic limit of 
the spherical Hankel functions and the plane-wave limit appear in the phase of the double scattered wave. We have also calculated (but not plotted) these scattering events with the hybrid renormalized homogeneous wave model described in section III: we find a curve roughly half way between the zero and first order results.

The surprising success of the first order Taylor expansion has an interesting origin which will lead to the third topic of this section, the MQNE description of the Taylor expansion. The first-order Taylor expansion is accurate because the origin-ghift addition theoren does not change the magnetic quantum number if the shift is parallel to the $z$ $\operatorname{axis}^{13}$. For the scattering geometry we selected, the outgoing photoemission wave has $m=0$ along the electric vector. Since the scattering atom vector is nearly parallel to $\vec{\varepsilon}$, the scattered partial waves will also have $m=0$, even if they now have $l$ from 0 to $l_{\max }$. Encountering the sulfur atom and scattering into the detector will give double scattered partial waves also with $m=0$ along $\vec{\varepsilon}$. Thus to a fair approximation we need only $m=0$ waves for the entire problem.

What of a more general geometry? Consider, for example, scattering first from the Ni atom directly behind the sulfur atom followed by scattering from another nearest neighbor $\mathrm{Ni}$ atom. Then the second scattering vector, $\vec{b}$, will no longer lie parallel to $\vec{a}$. To use the result that $m$ will not change for $z$ axis shifts we must rotate the $m=0$ partial waves emanating from the $N i$ aton at $\vec{a}$ to the $z|| b$ system. This rotation will include all magnetic sublevels $-\ell \leq m^{\prime} \leq l$ in the $z|| b$ system in proportion to the overlap integral (rotation matrix element) between 
spherical harmonics in the two systems. These manifold sublevels are not, however, equally effective in scattering from the second $\mathrm{N} I$ atom. As illustrated in Fig. 9, the $m=0$ spherical harmonics overlap the potential along the scattering bond length, $m=1$ waves overlap the potential farther from the axis and so on until some $m=\tau$ sublevel does not overlap the potential at all. Thus only the $\tau$ lowest magnetic sublevels need be overlapped with the $m$ - 0 waves and--by Nozawa's result--only the $\tau$ lowest sublevels will appear on center $b$ as scattered waves. For a triple scattering event, these lowest $\tau$ sublevels will need to be rotated to $\tau$ sublevels along the new scattering axis. Hence we identify the rotated-frame magnetic sublevels with the $q$ index in the Taylor expansion model.

We can push this picture farther by comparing the classical orbits sketched in Fig. 3 to the incident spherical harmonics in Fig. 9. The largest magnetic sublevels only overlap the outer regions of the potential, regions which contribute to forward scattering, not backscattering. This would suggest, again, that the Taylor series will converge much more quickly for backscattering.

\section{CONCLUSION}

We have explored $f$ ive small atom approximations. Some specific points bear summarizing here:

1) the success of $\mathrm{plane}$ wave models relies on backscattering geometries, 
ii) the plane-wave model requires incident waves at their asymptotic imit and a small diameter potential; it is inappropriate for multiple scattering calculations in solids,

iil) the point-scattering and homogeneous-wave models are Inadequate for multiple-scattering in the intermediate energy range, at least for near neighbor scattering,

iv) the homogeneous-wave model is the zero-order Taylor series term,

v) the Taylor series model allows methodical improvement in scattering calculations, and it follows from physically appealing magnetic quantum number expansion picture of the scattering partial waves.

We have also developed the multiple scattoring equations for ARPEFS with the Taylor series expansion of the orisin-saift addition theorem and illustrated the results with a two atom model.

The most direct extension of this work would be the application of the Taylor expansion method to simulations of experimentally measured ARPEFS curves. In addition to the elastic, multiple scattering equations derived here, we must also include important inelastic soattering factors and effects such as finite aperture integration before quantitative agreement with experiment could be expected. Under the appropriate development, the MQNE origin-shift addition theorem will also give multiple scattering models for other spectroscopies based on electron scattering in the intermediate energy range. EXAFS should yield to a low order expansion since the multiple 
scattered wave must always return to the absorbing atom: forward scattering will necessarily be coupled with backscattering as in the example in section VI. Electron diffraction in the 100-600 eV range should also be amenable to the treatment given here with the direct wave replaced by the incident plane wave. The first scattered wave will, of course, then be given exactly by the plane wave scattering factor.

The Taylor series expansion itself deserves further exploration. Accurate error bounds would eliminate empirical verification of convergence. Alternate parameterization of the scattering factors might reduce the computation burden required for the scattering calculations. The magnetic quantum number expansion pleture suggests that a variation of the equations presented here could be blilt up from rotation matrices and Nozawa's origin-shift formulas. Finally, the formulation of the exact multiple scattering equations (matrix inversion method) with the Taylor series result should be examined. At least pairwise or colinear multiple scattering seems feasible but more conplex geometries would require detailed study. 
APPENDIX A. COMPARING NOTATION WITH REF. 4

We have deduced a formula equivalent to eqn. (37) for the exact curved wave scattering of $\ell=1$ waves in ref. 4; we demonstrate that equivalence here. The single-scattered wave from ref. 4 may be written

$\underset{\vec{a}}{\Psi_{\vec{a}}(\vec{R})}=\left(\frac{3}{4 \pi}\right)^{1 / 2} \frac{e^{i k|\vec{R}-\vec{a}|}}{1 k R} \frac{e^{i k a}}{a}\left\{d_{1}(k a) \cos \theta_{\varepsilon a} f_{a R}^{00}\right.$

$$
-i \cos \theta_{\varepsilon a} \rho_{a R}^{10}-\frac{i}{k a} \sin \theta_{\varepsilon a} \sin \theta_{a R} \cos \phi_{\varepsilon a R} f_{a R}^{01}
$$

where

$$
f_{a R}^{n m}=\frac{1}{i k} \sum_{\ell=0}(2 \ell+1) T_{\ell}(k) \frac{\partial^{n} d_{\ell}(k a)}{(\partial k a)^{n}} \frac{\partial^{n} P_{\ell}\left(\cos \theta_{a R}\right.}{\partial\left(\cos \theta_{a R}\right)} .
$$

From our new definition we have

$\cos \theta_{\varepsilon a} F_{00}^{00}+\cos \theta_{\varepsilon a} F_{10}^{00}=\frac{\cos \theta_{\varepsilon a}}{1 k} \sum_{\ell=0}(2 \ell+1) T_{\ell}(k) P_{\ell}(\cos \theta)\left[H_{\ell}^{00}+H_{\ell}^{10}\right]$ and from Appendix C, ref. 13

$H_{\ell}^{00}+H_{\ell}^{10}=\left[\frac{\ell+1}{2 \ell+1}\right] d_{\ell+1}+\frac{\ell}{2 \ell+1} d_{\ell-1}$.

Using eqn. B9 in Ref. 4, the right side becomes

$$
H_{\ell}^{0} O+H_{\ell}^{10}=d_{\ell}-\frac{d_{\ell}}{1 k r}-i \frac{\partial d_{\ell}(k a)}{\partial(k a)}
$$


Working back through the definition of $\mathrm{f}_{\mathrm{aR}}^{00}$ and $\mathrm{f}_{\mathrm{aR}}^{10}$ shows that

$\cos \theta_{\varepsilon a} F_{00}^{00}+\cos \theta_{\varepsilon a} F_{10}^{00}=d_{1}(k a) \cos \theta_{\varepsilon a} e_{a R}^{00}-i \cos \theta_{\varepsilon a} P_{a R}^{10}$.

where $d_{1}(k a)=1+1 / k a$. Similarly we write out

$F_{01}^{00}(\vec{a}, \vec{R})=\frac{1}{i k} \sum_{\ell}(2 \ell+1) T_{\ell}(k) \frac{P_{\ell}(\cos \theta)}{(2 \ell+1)}\left[d_{\ell-1}-d_{\ell+1}\right]$

where a factor of $\ell(\ell+1)$ in $\mathrm{H}_{\ell}^{\mathrm{pq}}$ cancels the last factorial in the definition of $\mathrm{F}_{01}^{00}$. The connection between associated Legendre polynomials and derivatives of Legendre polynomials:

$P_{\ell}^{m}(\cos \theta)=\sin ^{m} \theta \frac{d^{m}{ }^{(\cos \theta)}}{d(\cos \theta)^{m}}$ and the recursion for $d_{\ell}$ gives

$\sin \theta_{\varepsilon a} \cos \phi_{\varepsilon a R} F_{01}^{00}(\vec{a}, \vec{R})=\frac{\sin \theta_{\varepsilon a} \cos \theta_{\varepsilon a R} \sin \theta_{a R}}{1 k r} f_{a R}^{01}$.

These close connections demonstrate that 1) the differential formula of ref. 4 could be used as a basis for a Taylor expansion, ii) the rotation matrix approach employed here for approximate origin shift will lead to compact exact origin shift formulas, and iif) individual scattering factors $F_{p q}^{00}$, and by extension $F_{p q}^{p^{\prime} q^{\prime}}$, can be interpreted as specific spherical wave corrections as described in ref 4 and 13. Our new formulation is recommended for numerical work. 
APPENDIX B. ON THE SIGN FACTOR $s_{q}^{q}$

The sign of the Taylor series scattering factor is a combination of the signs in the definition of $\mathrm{N}_{\ell q}$ :

$$
\begin{array}{ll}
(-1)^{q} & q \geq 0 \\
(1) & q<0,
\end{array}
$$

the factor of $\exp (1 \pi q)$ from the rotation matrix, and the symmetry relations for rotation matrices. Note that the symmetry relations are applied depending on the sign of $q-q^{\prime}$ and $q+q '$; we have four cases:

1) $q+q^{\prime} \geq 0, q-q^{\prime} \geq 0$

$$
r_{q^{\prime} q^{l}}^{\ell}(\beta)=r_{q^{\prime} q^{(\beta)}}^{\ell}
$$

ii) $q+q^{\prime} \geq 0, q^{\prime} q^{\prime}<0$

$$
r_{q^{\prime} q^{(\beta)}}^{\ell}=(-1)^{q+q^{\prime}} r_{q q^{\prime}}^{l}(\beta)
$$

iii) $q+q^{\prime}<0, q-q^{\prime} \geq 0$

$$
r_{q^{\prime}}^{\ell} q^{(B)}=r_{-q-q^{\prime}}^{\ell}(B)
$$

iv) $q+q^{\prime}<0, q-q^{\prime}<0$

$$
r_{q^{\prime} q^{l}}^{l}(\beta)=(-1)^{q-q^{\prime}} r_{q^{\prime}-q^{(B)}}
$$

In case $i 1 i)$ the factors of $(-1)^{q-q^{\prime}}$ cancel from successive symmetry relations; in cases $11 i$ ) and $i v$ ) the criterion for equation (44) to apply changes since equation (45) negates the indices. Surveying these cases shows that only when $\left(q-q^{\prime}\right)<0$ will factors of $(-1)$ be required.

To summarize these factors we note that

$$
\begin{aligned}
& |q|+q=0 \quad \text { if } \quad q \leq 0 \\
& =2|q| \text { if } q>0
\end{aligned}
$$

while 
163

$$
\begin{array}{rlrl}
\left|q-q^{\prime}\right|-\left(q-q^{\prime}\right) & =2\left|q-q^{\prime}\right| & & \text { if }\left(q-q^{\prime}\right) \leq 0 \\
& =0 & \text { if }\left(q-q^{\prime}\right)>0
\end{array}
$$

All of the factors may be written then as

$$
s_{q}^{q^{\prime}}=(-1)^{q}{ }_{i}\left|q-q^{\prime}\right|-\left(q-q^{\prime}\right)_{i}|q|+q_{i}\left|q^{\prime}\right|+q^{\prime} \text {, }
$$

or since $i^{4}\left|q^{\prime}\right|=1$,

$$
s_{q}^{q^{\prime}}=(-1)^{q}{ }_{i}\left|q-q^{\prime}\right|+|q|-\left|q^{\prime}\right|
$$




\section{ACKNOWLEDGEMENTS}

This work was supported by the Director, Office of Energy Research, Office of Basic Energy Sciences, Chemical Sciences Division of the U.S. Department of Eriergy under Contract No. DE-AC03-76SF00098. 
REFERENCES

1. J.J. Barton, C.C. Bahr, Z. Hussain, S.W. Robey, J.G. Tobin, L.E. Klebanoff, and D.A. Shirley, Phys. Rev. Lett. 51, 272 (1983).

2. P.A. Lee, P.H. Citrin, P. Eisenberger, and B.M. Kincaid, Rev. Mod. Phys. 53, 769 (1981).

3. B.K. Teo, J. Am. Chem. Soc. 103, 3990 (1981); J.J. Boland, S.E. Crane, and J.D. Baldeschwieler, J. Chem. Phys., 77, 142 (1982).

4. J.J. Barton and D.A. Shirley, "Curved Wavefront Corrections for Photoelectron Scattering," LBL-18692, and submitted to Phys Rev. B

5. J.B. Pendry, Low Energy Electron Diffraction, Academic Press, London, (1974).

6. S.Y. Tong, C.H. Li, "Diffraction Effects in Angle-Resolved Photoemission Spectroscopy" in Chemistry and Physics of Solid Surfaces V.III, CRC Press, Boca Raton, Florida, 1982.

7. B.A. Bunker and E.A. Stern, Phys. Rev. B 27, 1017 (1983).

8. P.A. Lee and J. B. Pendry, Phys. Rev. B 11, 2795 (1975).

9. V.A. Biebesheimer, E.C. Marques, D.R. Sandstrom, F.W.Lytle, and P.B. Greegor, J.Chem. Phys., 81, 2599 (1984).

10. C.H. L1 and S.Y. Tong, Phys. Rev. Lett. 43, 526 (1979).

11. P.J. Durham, J.B. Pendry, and C.H. Hodges, Comp. Phys. Comm. 25, 193 (1982).

12. C. Cohen-Tannoudji, B. Diu, F. Laloë, Quantum Mechanics, John Wiley and Sons, New York, 1977. 
13. J.J. Barton, and D.A. Shirley, "Approximate Translation of Screened Spherical Waves," LBL-19076, and submitted to Phys Rev. A., and Chapter' 3.

14. R. Nozawa, J. Math. Phys. I, 1841 (1966).

15. P.A. Lee, Phys. Rev. B 13,5261 (1976).

16. P.J. Orders and C.S. Fadley, Phys. Rev. B 27, 781 (1983).

17. L.I. Schiff, Quantum Mechanics, 3rd edition, McGraw-Hill, New York, 1968.

18. J.A. Gaunt, Phil. Trans. Roy. Soc. (London) A228, 151, (1924).

19. A. Messiah, Quantum Mechanics, John Wiley and Sons, New York (1958).

20. M. Abramowitz and I.A. Stegun, Handbook of Mathematical Functions, National Bureau of Standards, Appl. Math. Services 55, (1964).

21. L. McDonnell, D.P. Woodruff, and B.W. Holland, Surf. Sci. 51, 249 (1975).

22. H. Daimon, H. Ito, S. Shin, and Y. Murata, J. Phys. Soc. Japan, 53, 3488 (1984).

23. A.R. Edmonds, Angular Momentum in Quantum Mechanics, 2nd ed. Princeton Uni versity Press, Princeton, NJ (1960).

24. B.A. Bunker and E.A. Stern, Phys Rev. B, 27, 1017 (1983). 


\section{Figure Captions}

Figure 1. Definition of the vectors used in our scattering equations. The electric vector is represented $\lrcorner y \hat{\varepsilon}$. We place the origin at the photo-emitter, tne detector lies along $\vec{R}$. Vectors $\vec{a}$, $\vec{b}$, and so on run from one scattering atom to the next.

Figure 2. Schematic illustration of four small-atom approximations described in Section III, plotted along the scattering vector $\vec{a}$. Every panel contains, as the dotted curve, a graph of the imaginary part of the spherical Hankel function for $\ell=7, k$ $=8 \mathrm{~A}^{-1}$. The abscissa $\mathrm{gives}$ the distance from the wave function origin in A. Each panel also contains an arrow centered at $2.23 \AA$, the $S-N I$ bond length for $c(2 \times 2) S / N i(100)$, to indicate the extent of a $\mathrm{NI}$ atom potential of effective radius $0.8 \AA$. (a) Plane-wave model, functional dependence agrees with spherical wave but has errors in phase and amplitude. (b) Point-scattering model, phase and amplitude correct at $r=2.23 \mathrm{~A}$, errors in both at the edges of the $\mathrm{Ni}$ potent1al. (c) Homogeneous-wave model, correct in $(1 / \mathrm{kr})$ dependence of amplitude, errors in phase, some small errors in amplitude at edge of $\mathrm{N} 1$ potential. (d) Hybrid, renormalized homogeneous-wave method, substantially correct over the range of the potential, some error at the small $r$ edge of the $\mathrm{Ni}$ atom. 
Figure 3. Schematic semiclassical orbits for an attractive potential. If the circle represents the effective radius of a screened nuclear charge, then particles with large impact parameters will sample only the weak outer region of the potential and scatter. through small (forward) angles. Particles with small impact parameters orbit the strong nuclear attraction and exit at large (backscattering) angles. The connection to wave scattering is made through $b=\ell / k$ where $b$ is the impact parmeter: large $\ell$ partial waves contribute to forward scattering and small $\ell$ waves dominate for backscattering.

Figure 4. Taylor series scattering factor amplitude at $k=8 \AA^{-1},|\vec{a}|=$ 2.23A, as a function of scattering angle, $\theta_{a b}$. The scattering factors generally have similar shapes whenever they agree in $\left|q-q^{\prime}\right|$ and $|q+q \cdot|$; hence we will only plot representative examples. We adopt the notation $\left(p^{\prime} q^{\prime} \mid p q\right)$. (a) solid line $(00 \mid 00)$, an example of factors with $\left|q-q^{\prime}\right|=$ $0,|q+q|=0$. This is the single zero order factor. (b) circles $(01 \mid 10),|q+q \cdot|=1,\left|q-q^{\prime}\right|=1$. (c) crosses $(02 \mid 20),\left|q+q^{\prime}\right|=2,\left|q-q^{\prime}\right|=2$. Factors are multiplied by 3 after the break at $60^{\circ}$; the right hand scale applies to this region.

Figure 5. Same as figure 4; note the increased scales. (a) solid line $(00 \mid 01),\left|q-q^{\prime}\right|=1,\left|q+q^{\prime}\right|=1 ;$ (b) circles $(01 \mid 01),\left|q-q^{\prime}\right|$ 
$=0,\left|q+q^{\prime}\right|=2(c)$ crosses $(02 / 01),\left|q^{\prime} q^{\prime}\right|=1\left|q+q^{\prime}\right|=3$;

(d) plus symbols $(02 / 02),\left|q-q^{\prime}\right|=0,\left|q+q^{\prime}\right|=4$.

Figure 6. Same as figure 4. (a) solid line (11/20) $\left|q^{\prime}-q^{\prime}\right|=1,\left|q+q^{\prime}\right|$

- 1. (b) circles $(01 / 1-1),\left|q-q^{\prime}\right|=2,\left|q+q^{\prime}\right|=0$. (c) crosses $(02 / 1-1),\left|q-q^{\prime}\right|=3,\left|q+q^{\prime}\right|=1$. (d) plus symbols $(02 / 0-2),\left|q-q^{\prime}\right|=4,\left|q+q^{\prime}\right|=0$.

Figure 7. ARPEFS oscillations calculated by exact Gaunt integral summation (thick curve) and plane wave approximation (thin curve). These curves simulate the fractional oscillation of the $S$ (1s) partial cross-section from $c(2 \times 2) S / N i(100)$ along [110], but consider only a single $\mathrm{Ni}$ atom scatterer. The inget diagram 1llustrates the three waves which sum to give the photoemission final state, the direct, single-scattered, and double-scattered waves. The backscattering angle is $173^{\circ}$; the forward angle is $7^{\circ}$. Both curves have been multiplied by $\exp \left(-0.02 k^{2}-2.23 / .173 k\right.$ ) (Debye waller and Inelastic attenuation) to give a more realistic amplitude comparison.

Figure 8. Same as figure 7 except comparing the exact Gaunt summation to the zero order Taylor series result. The first order Taylor result cannot be distingulshed from exact, on this scale. 
Figure 9. Schematic illustration of the magnetic quantum number expansion interpretation of eqn. (31). A polar plot of the four lowest magnetic sublevels of a $\ell=7$ spherical harmonic is superimposed upon a circle whose radius represents the effective radius $r_{0}$ of a nearest neighbor potential. The line connecting the incident wave source and the potential origin is used for the spherical harmonic polar axis and only the region of angles near the pole is plotted. The angle functions have been rescaled to place their first maxima on the same radius. The $m=0$ sublevel (solid line) is seen to overlap the strong central portion of the potential, while the $m=1$ lobes (dotted line) peaks further from the axis. The $m=2$ lobes (dot-dash lines) only intercept the $f a r$ edges of the potential and the $m=3$ level (dashed lines). completely missed the mark. 


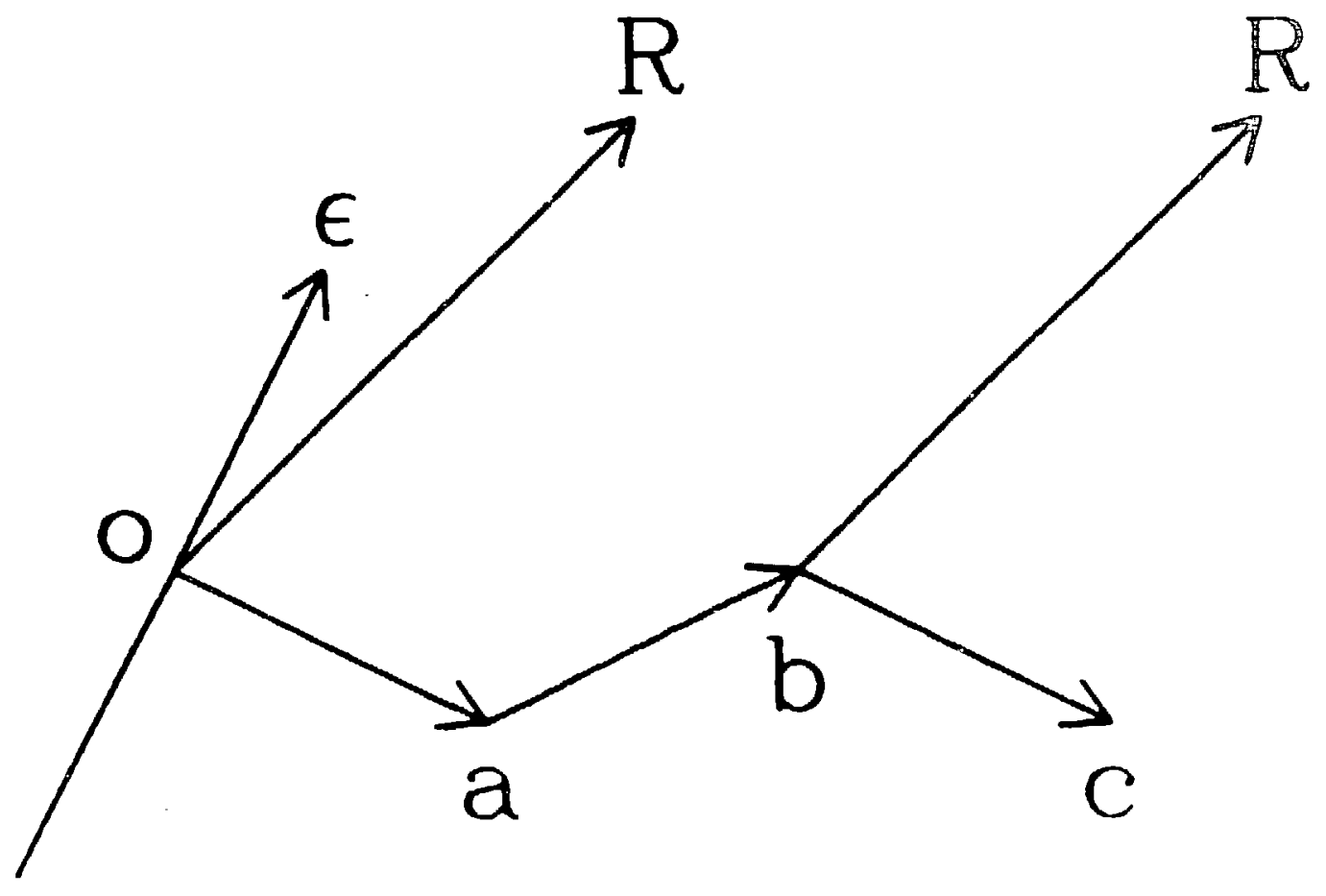

XBL 851-1020

Figure 1 


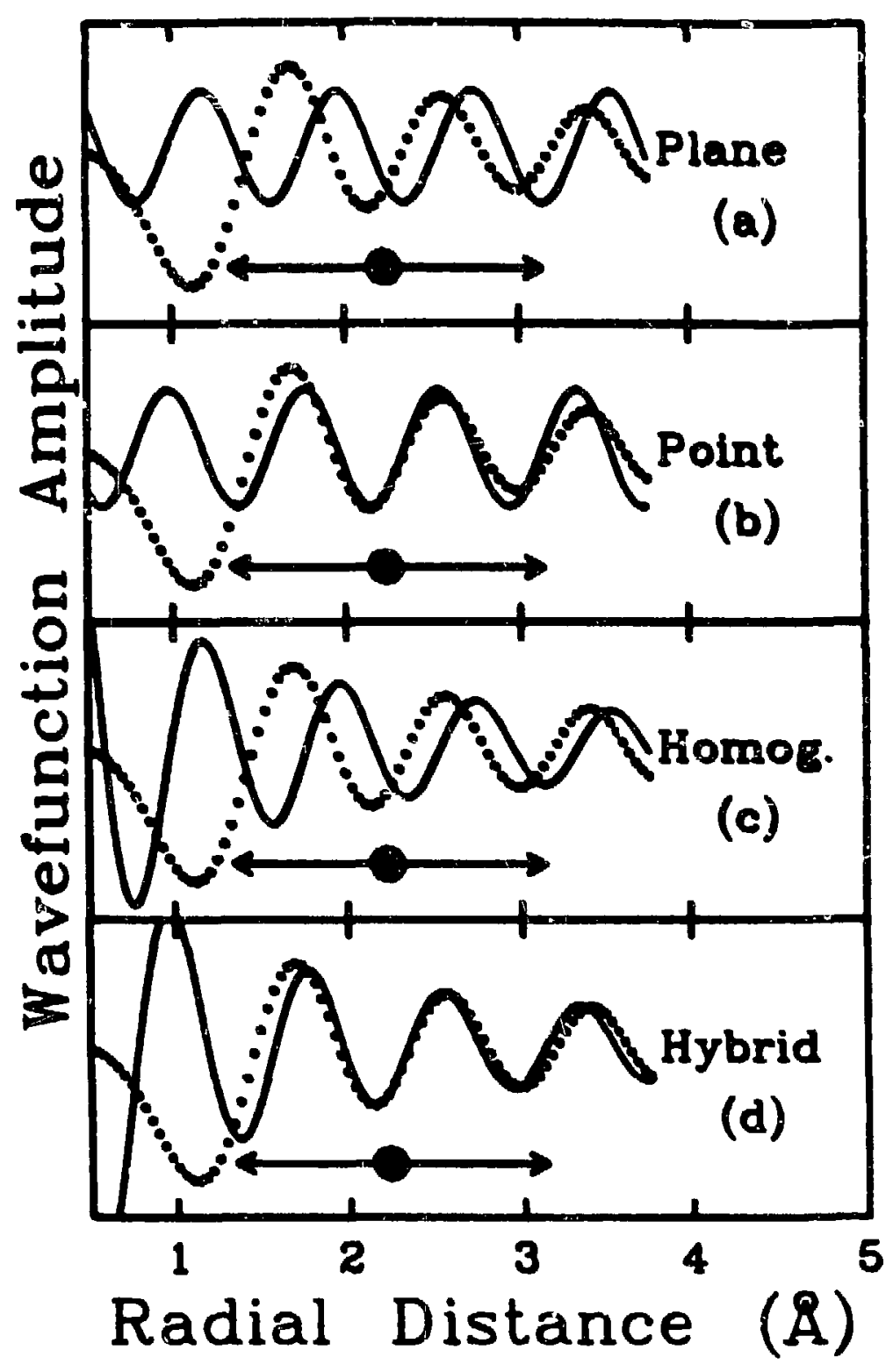

XBL 851-1022

Figure 2 


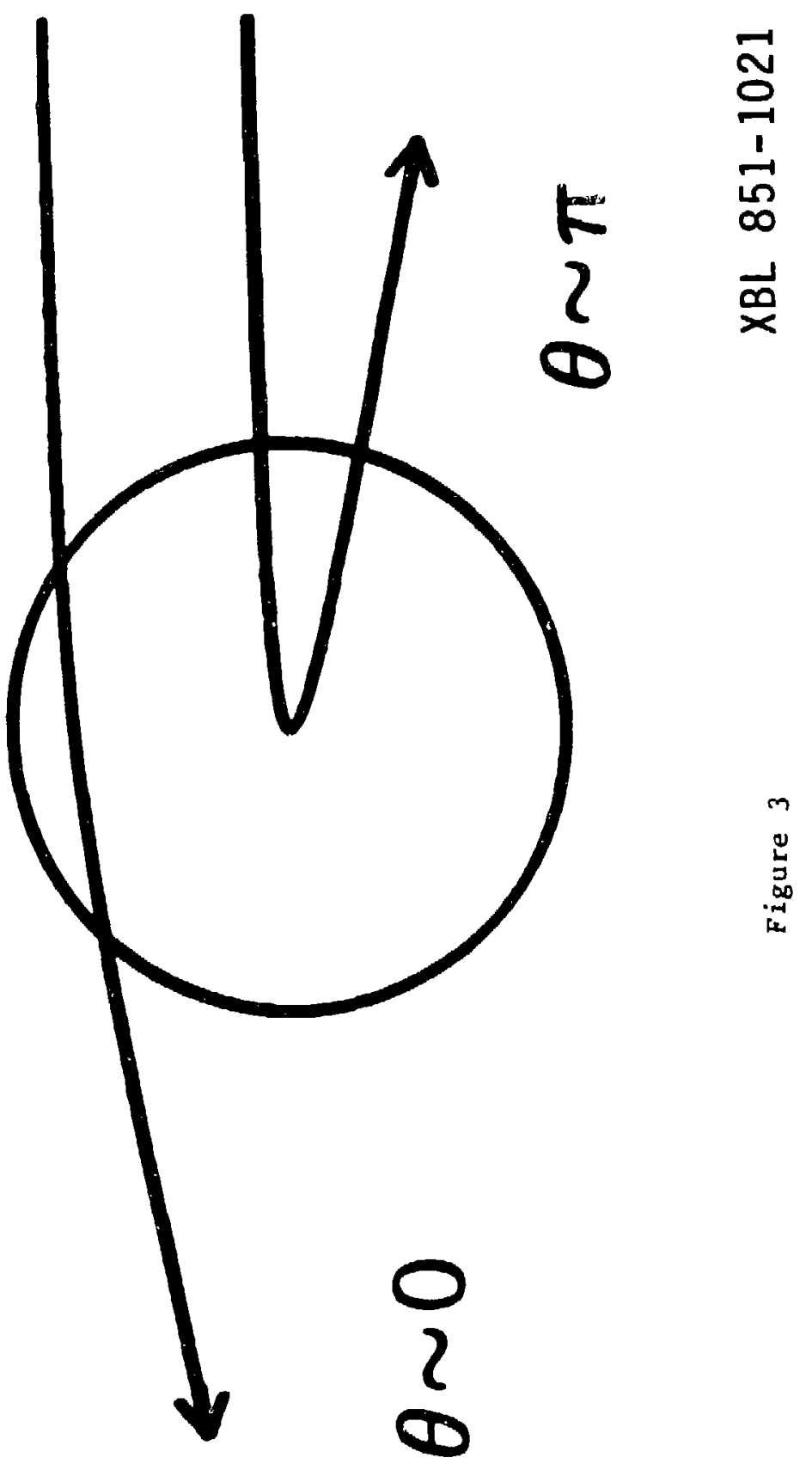


$\mathrm{Ni}$ Atom $\left(k=8 \AA^{-1}\right)$

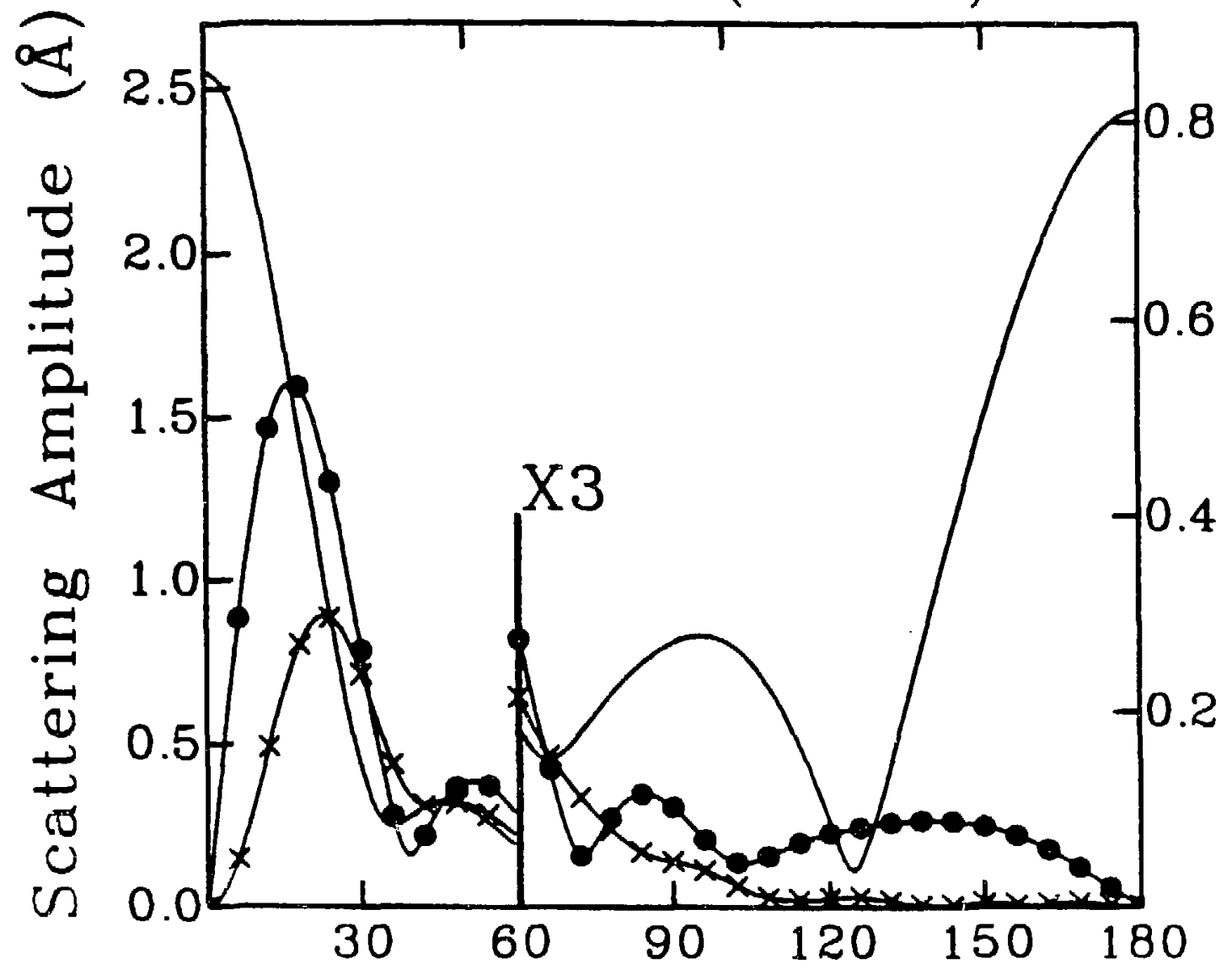

Scattering Angle (degrees) 
$\mathrm{Ni}$ Atom $\left(k=8 \AA^{-1}\right)$

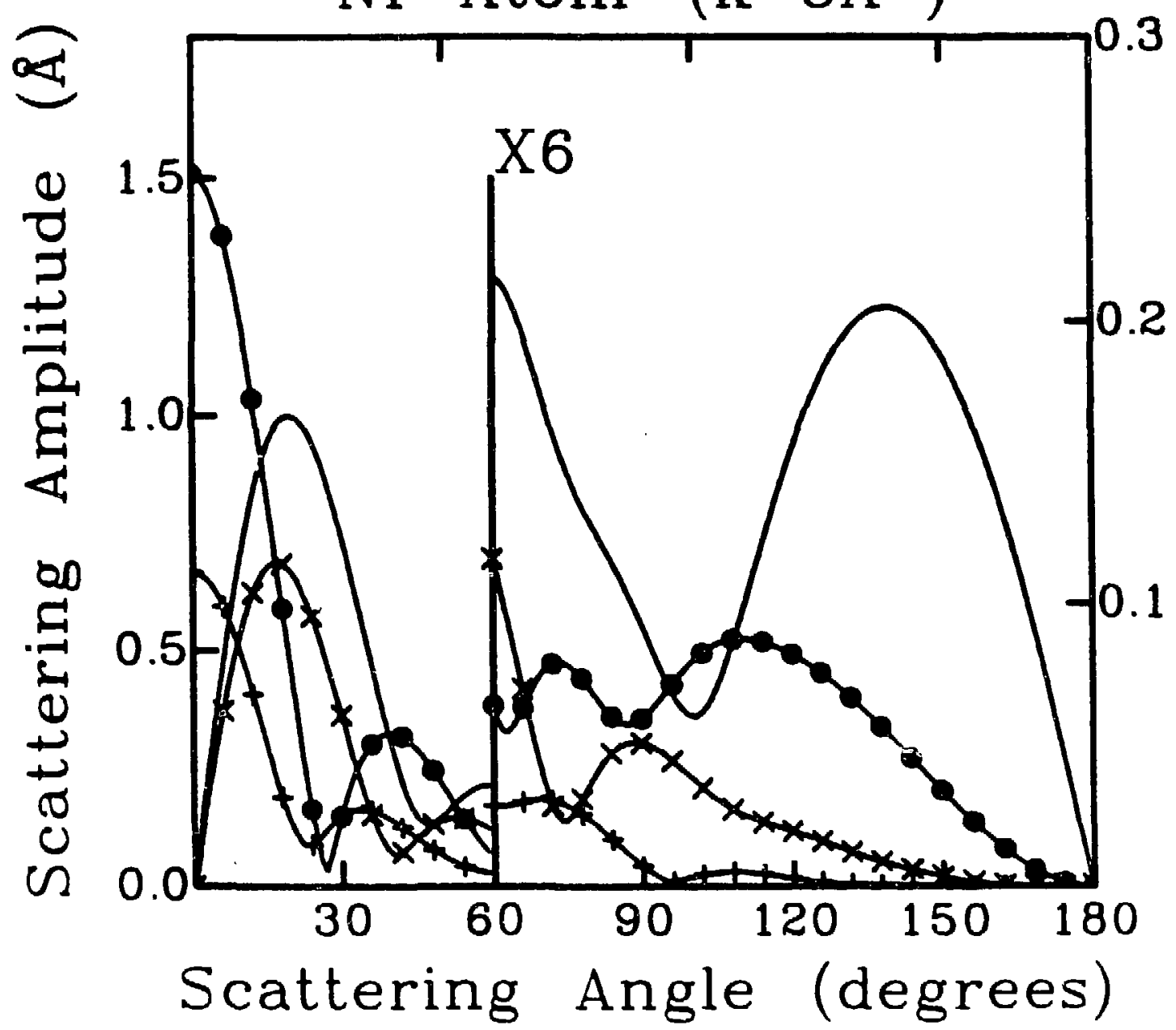

XBL 851-1016

Eigure 5 
$\mathrm{Ni}$ Atom $\left(k=8 \dot{\AA}^{-1}\right)$

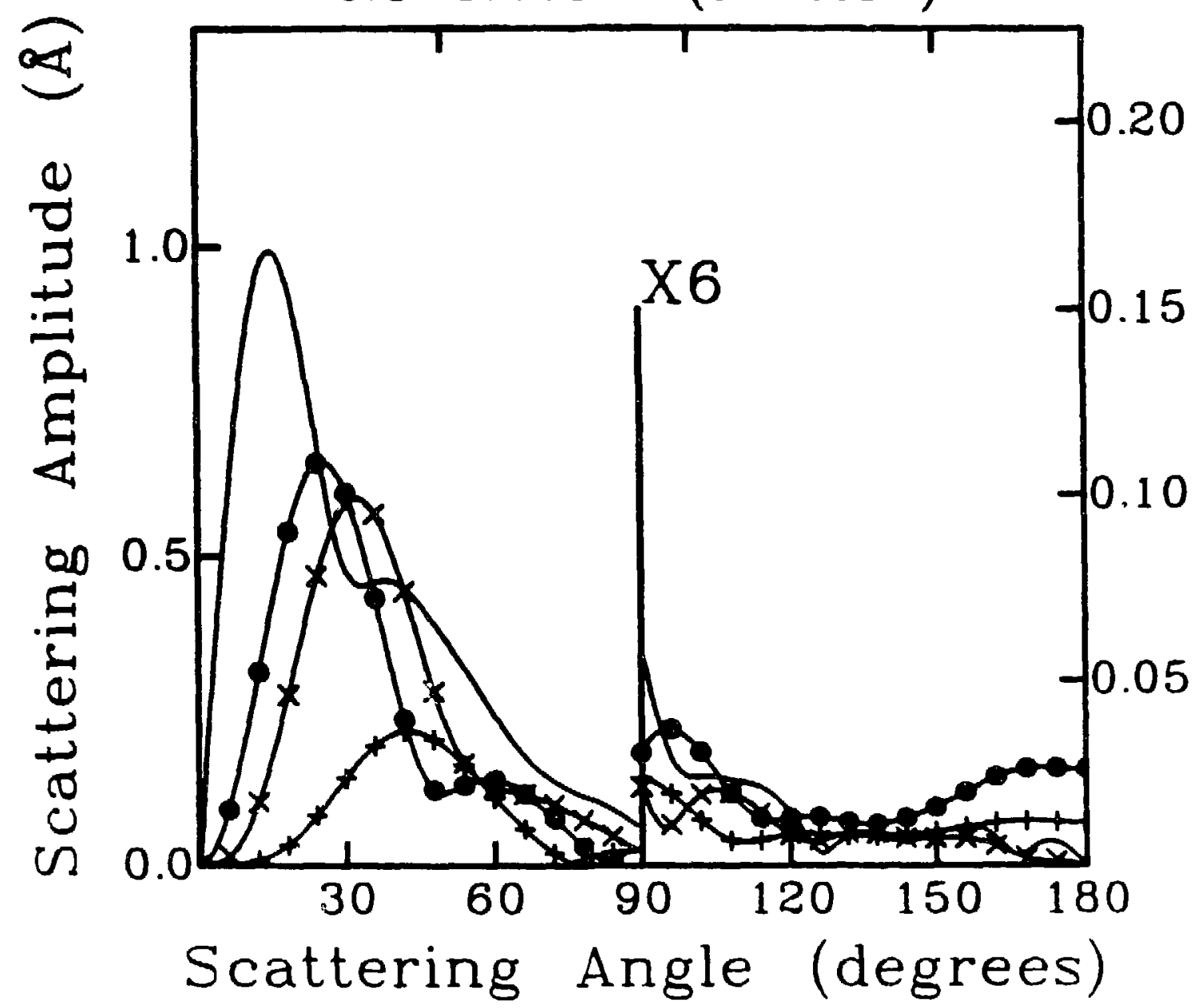

XBL 851-1018

Figure 6 


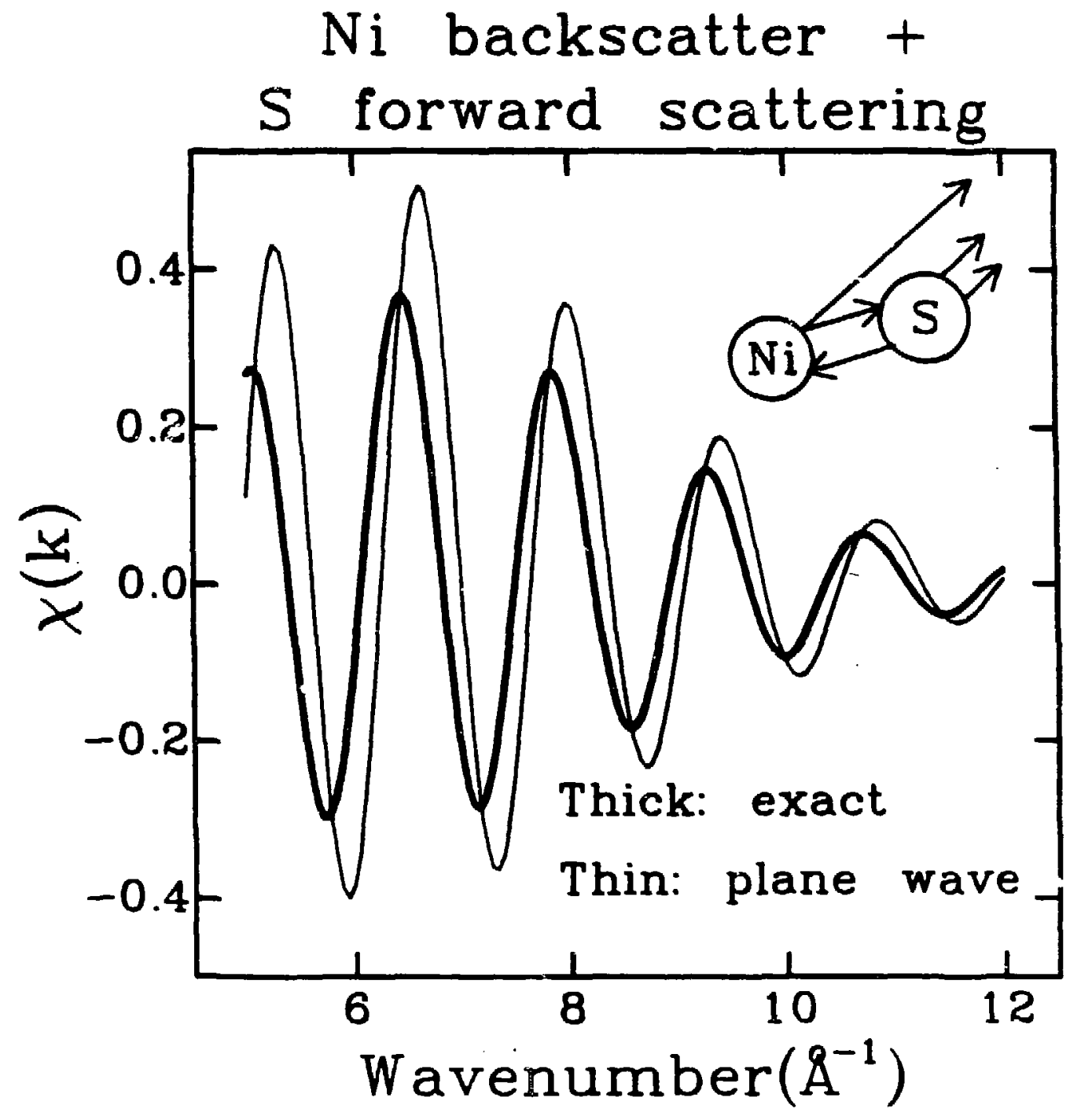

XBL 851-1023

Figure 7 


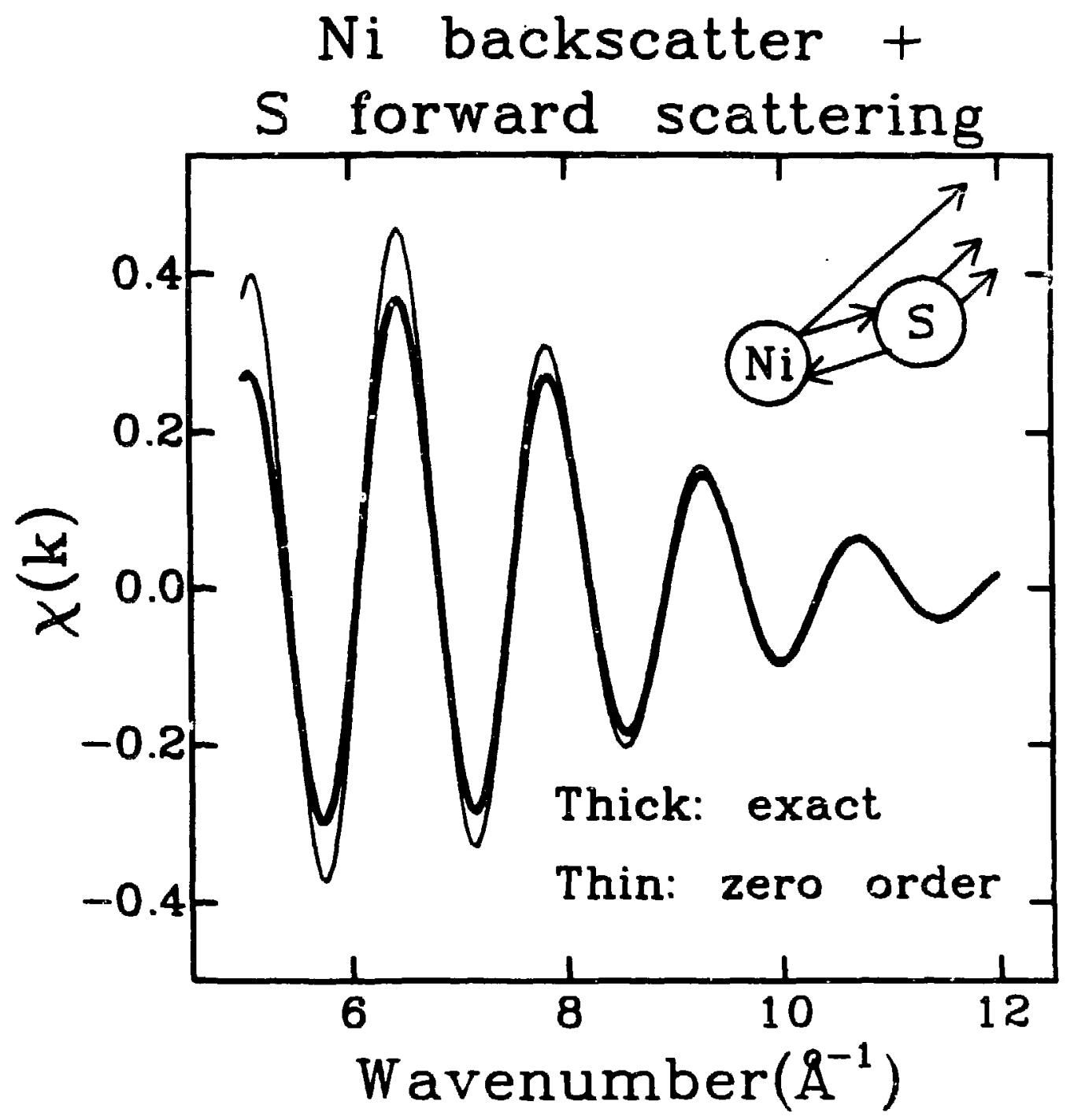

XBL 851-1024

Figure 8 

CHAPTER 5:

\section{Theory of Angle-Resolved Photoemission Extended Fine Structure}

\section{APSTRACT}

We outline the form of a theory for photoelectron scattering in the 100-1000 eV energy range to simulate experimental measurements of AngleResolved Photoemission Extended Fine Structure (ARPEFS) from ordered crystalline solid surfaces. The problem is divided into a zero-order problem of photoabsorption in the presence of the solid's valence electron density and a scattering problem which incorporates the scattering ion-cores in a perturbation series (cluster expansion). The dynamics of core-hole relaxation are discussed but the dynamic effects are shown to be small. The Taylor-series magnetic quantum number expansion is used for the curved-wave, multiple-scattering equations. Surface barrier refraction is discussed, but we argue that a velocitydependent surface barrier gives only an inner potential shift with no surface electron refraction. Analytic formulas for aperture integration are derived and discussed; thermal averaging in a correlated Debye model is extended to multiple scattering and scattering amplitude averaging is included. Reasonable values for non-structural parameters in the theory are shown to give good simulations of the experimental ARPEFS measurements from $\mathrm{c}(2 \times 2) \mathrm{S} / \mathrm{Ni}(001)$. We find, in agreement with full multiple-scattering calculations, that forward focussing is a fundamental feature of ARPEFS and that curved-wave corrections are essential for semi-quantitative reslits. Since the scattering path 
length difference is not alltered by forward scattering, the ARPEFS oscillation frequency is nearly equal to the geometrical path length difference but the amplitude and constant phase of the oscillations cannot be predicted by single scattering theory. 


\section{INTRODUCTION}

Much of the interest in adsorption studies on clean, single-crystal surface relies on the usefulness of these systems as well characterized models for more complicated interfaces. Unfortunately, characterization of even these model systems has proven difficult. We have been exploring ${ }^{1,2}$ a new approach to determine surface structures with corelevel, angle-resolved photoemission. Core-level photoemission provides an element-specific, surface-sensitive, localized probe for adsorbates. By employing angle-resolved photoemission with polarized light we may independently orient the emission and polarization vectors: we can view the surface structure from many different angles and emphasize different atoms. These distinctive features of photoemission have lead to a number of experimental measurements and theoretical analysis 3,4 of surface structures, primarily by means of two measurement techniques, azimutial photoelectron diffraction (APD) and normal photoelectron diffraction (NPD). Recently, we reported ${ }^{1}$ the first structure determination using a new photoelectron diffraction technique which we call angle-resolved photoemission extended fine structure (ARPEFS). In this paper we will describe a theoretical nodel which provides the basis for analyzing ARPEFS measurements to extract surface structure information.

Experimental measurements of ARPEFS are very similar to those of normal photoelectron diffraction. An ordered overlayer, adsorbed onto a single crystal, is irradiated with soft $x$-rays from a tunable, monochromatic photon source. The photon energy is scanned from 50 to $500 \mathrm{eV}$ above some core energy level characteristic of the overlayer. At 
each energy, the photoemission intensity in a carefully selected emission direction is recorded. The resulting curve of Intensity versus electron kinetic energy contains oscillations we call ARPEFS. These measurements differ from NPD measurements only in their higher and wider energy range and in the unrestricted choice of emission angles.

The more significant differences between NPD and ARPEFS lie in the interpretation of the measurements, specifically, in the process of extracting the surface structure information. NPD was viewed as being closely related to low energy electron diffraction (LEED); the measured oscillations were ascribed to multiple scattering interferences and analyzed by trial-and-error comparison to sophisticated calculations. 4 ARPEFS is viewed as closely related to extended $x$-ray absorption fine structure (EXAFS); the scattering interferences seem to be directly related so individual scattering atoms and the scattering geometry may be extracted by Fourier analysis. ${ }^{1}$ One important goal in this paper is to examine the justifications for this simplified view of ARPEFS.

The physical explanation for ARPEFS is based on elastic electron scattering. Core-level photoabsorption gives a localized, atomic-like outgoing photoelectron wave. Direct propagation of this wave into the detector gives the overall atomic-like cross section to the ARPEFS curve. Propagation of this wave to the core region of nearby atoms creates a second set of elastically scattered waves which can also reach the detector. Interference between these two sets of waves give rise to the ARPEFS. Since the interference extrema occur for electron wavenumbers which are integral multiples of $\pi$ divided by the difference In path lengths for direct and scattered waves, the path length--and hence the geometry--can be determined from the oscillation frequency. 
This is the physical picture presented by Lee although he noted that the same physics had been described earlier ${ }^{6}$ and McDonnell ${ }^{7}$ et al had analyzed angular distributions of Auger emission with an equivalent model. Lee drew strong parallels between EXAFS and angle-resolved photoemission, further suggesting that Fourier analysis might be used in the analysis of angle-resolved photoemission. This localized, singlescattering cluster model was not thought to be adequate for the analysis of normal photoelectron diffraction data. ${ }^{4,5}$ Instead, the full multiple scattering analysis used for LEED was adapted to photoemission, first by Leibsch $^{8}$ and later and more extensively by Tong and coworkers. ${ }^{4}$ The success of this adaptation is evident in a series of surface structure analysis based on this approach. 3,4

Despite the success of the NPD analysis, the complexity of the theoretical analysis is discouraging. The first step toward a simpler technique came when Hussain, et al ${ }^{9}$ applied Fourier analysis to theoretically generated, wide energy range NPD curves. Hussain was able to relate peaks in the Fourier amplitudes to the interplanar spacing of adsorbate and substrate layers. While this would suggest that some simple model would predict the NPD curve, it would also seem to rule out Lee's model of localized scattering. Lee's model would seem to predict shells of near neighbor distances in the Fourier transform, rather than the interplanar distances observed. Orders and Fadley ${ }^{10}$ resolved this dilemma by investigating in detail a single scattering cluster model similar to Lee's. They noticed that the strong peaking of the scattering angular distribution for backscattering in the intermediate (100-600 ev) energy range would highlight eubstrate atoms directly below the adsorbate in the NPD normal emission geometry. Thus, the localized 
EXAFS-like theory could explain the Fourier transform result without resorting to a multiple scattering model.

With the basic form of the localized cluster theory reconciled with the NPD theoretical curves, we measured ${ }^{1}$ intermediate energy off-normal photoelectron diffraction data for $\mathrm{c}(2 \times 2) \mathrm{S} / \mathrm{N} 1(100)$ and applied the techniques of EXAFS analysis in an attempt to derive the surface structure. To emphasize the differences between these measurements and their analysis and the NPD measurements and their multiple scattering analysis, we have called the new technique angle-resolved photoemission extended $f$ ine structure (ARPEFS).

The success of Lee's model as a basis for interpreting the ARPEFS to extract structure does not follow from the quantitative accuracy of numerical calculations with the model. In fact, numerical calculations by Bullock, Fadley, and Orders ${ }^{11}$ demonstrate that the basic singlescattering theory reproduced only the barest outlines of the experimental results. From the experimental measurements and their Fourier transforms, it appears that only backscattering atoms and nearest neighbor atoms contribute substantially to the ARPEFS curve, Many other atoms contribute to the theoretical curves, giving the simulated measurements $(X(E))$ too much structure and the theoretical Fourier transform far too many peaks. Although Bullock, et al. concluded from their calculations that a great many scattering atoms must be considered when analyzing ARPEFS, we believe their conclusions are valid only for their single-scattering theory curves and do not apply to the experlinental measurements.

As the development of EXAFS 1llustrates, ${ }^{12}$ only a semi-quantitative model is necessary when the measured signal is sinusoidal with a 
frequency dominated by geometrical path length differences. However, an accurate theory can forestall errors or misunderstandings inherent in a purely empirical model and confirm--or refute--models for the underlying physics of electron scattering. Furthermore, a complete theory will suggest new experimerial techniques or new information which may be derived from existing measurements. Thus we take up the theory of ARPEFS.

Our ultimate goal for the theory is a simulated curve which approaches the measured data points to within their experimental precision. If a theory fails this test we could seek improvements by changes in 1) the form of the theory, 11) non-structural parameters in the theory, or iii) structural parameters in the theory. These areas are, of course, tightly coupled, but in this paper we will concentrate solely on the form of the theory. Although we know better, we will assume that the non-structural parameters and the structure itself are well understood from independent sources.

Furthermore, our treatment will be more complete in its statement of problems than 1 will be in proposed solutions. We attempt to survey the whole theory, giving each part only that consideration essential to the determination of surface structures with ARPEFS. We believe a more thorough study of several problems described here would be useful and interesting, but our specific goal in this paper is to clarify the issues.

Some of the ingredients of this ARPEFS theory have already been presented before. Beyond the qualitative analysis of Lee ${ }^{6}$ and McDonnell et al's early Auger results ${ }^{7}$, theories which describe photoelectron scattering in the intermediate energy range have been 
developed by Fadley and coworkers. $10,11,13,14$ Fujikawa, ${ }^{15}$ and Tong and coworkers. 4

Bullding on earlier work in azimuthal photoelectron diffraction, 14 Orders and Fadley ${ }^{10}$ applied a single scattering cluster model to normal enission ARPEES. Their calculation includes:

1) single scattering,

11) Plane waves,

1i1) full partial-wave expansion of the scattering potential,

iv) uncorrelated Debye-Waller vibrational averaging,

v) isotropic, static mean free path,

vi) surface refraction, and

vii) numerical aperture integration.

Individual scattering events are sufficlently simple in this formulation that important insights could be gained about the nature of the electron scattering, specifically the connection between scattering anisotropy and interlayer distances. Unfortunately, this model is not adequate for detailed calculations and its application to ARPEFS by Bullock, Fadley, and orders ${ }^{11}$ served primarily to spur further work. Very recently, Sagurton, Bullock, and Fadley ${ }^{13}$ redeveloped this model to include spherical wave scattering and correlated Debye Waller factors, giving considerable improvements in comparison to experiment.

The theory described by Tong et al. is complementary to the Fadley group. ${ }^{4}$ Their methods are those developed for NPD studies in the low electron energy regime and extended, with some approximations, to intermediate energies. Their calculations include: 
1) multiple scattering,

ii) spherical waves,

iii) truncated partial-wave expansion of the potential,

iv) vibrational correction via temperature dependent scattering factors (uncorrelated Debye model),

v) isotropic, static mean free path via a complex inner potential,

vi) refraction, and

vii) no aperture integration.

The sophistication of these calculations has limited their use either for simulation of experimental curves or for further understanding of the photoelectron scattering. Nevertheless this model led to two important results of particular note: i) the aforementioned Fourier transform results of Hussain, et al ${ }^{9}$ providing the link with earlier NPD results and ii) the "quasi-dynamic" multiple scattering method. This latter result is equivalent to our conclusion that multiple scattering serves to focus the single-scattered waves without introducing new pathlength differences: the quasi-dynamical calculation includes all single scattering paths plus all forward multiple scattering paths.

The theory presented by Fujikawa ${ }^{1.5}$ is a full spherical-wave, multiple-scattering, cluster method, but it has not to our knowledge been applied to any photoelectron scattering such as we are interested in here, and we will not discuss it further.

Our work falls somewhat between that of the Fadley and Tong groups. In related papers we have investigated the nature of curved wave 
corrections to the single scattering of photoelectrons ${ }^{16}$ and disived ${ }^{17}$ new approximate formulae for the multiple scattering of spherical waves by a method which we will refer to here as the Taylor series magnetic quantum number expansion (TS-MONE). Our most significant contribution to the theory of ARPEFS will then be application of the TS-MQNE multiple scattering equations and the qualitative insight from ref 16 to arrive at a complete but parsimonious account of the elastic scattering of photoelectrons. Thus we include all the scattering events considered by Tong's curved wave, quasi-dynamic theory, but we retain the relative simplicity of the cluster approach. To treat inelastic damping, we adapt a dynamic but isotropic mean free path damping from EXAFS work. We include the correlated Debye Waller vibrational correction extended to multiple scattering and show how to include the vibrational averaging of the scattering amplitude. We derive an analytic formula for the angle :ategration effect. For comparison, our model contains:

1) multiple scattering,

11) spherical waves,

i1) full partial-wave expansion of the potential,

iv) correlated Debye-Waller factors,

v) a crude dynamic correction for mean free path,

vi) no electron refraction, and

vii) analytic aperture integration. 
II. GOALS OF THE THEORY; THE MODEL SYSTEMS

We begin by posing the theoretical problem to be solved. As stated in the introduction we wish to reproduce the experimental measurements. Since we are not yet prepared to critically examine the parameters in the theory we will not adopt formal statistical criteria for success. We will discuss the calculation of the ARPEFS spectrum, $X(E)$, but note that Fourler analysis can be used to extract structure information in a manner less sensitive to the theory.

We will specifically discuss the S(is) ARPEFS from $c(2 \times 2) S / N i(100)$ measured along [110] and [001] directions reported previousl $y^{1,2}$ and analyzed in detall in ref. 18. Using a procedure discussed in ref 18 , the experimental angle-resolved photoemission partial intensities are reduced to the proportional cross-section oscillations:

$x(E, \hat{R}, \hat{E})=\frac{I(E)-I_{0}(E)}{I_{0}(E)}$

where $E$ is the measured photoelectron kinetic energy. The curve $I(E)$ has been corrected for such effects as photon flux; $I_{0}(E)$ is taken as the smooth, slow varying part of $I(E)$. The unit vector $\hat{R}$ specifies the position of the photoemission analyzer and $\hat{\varepsilon}$ gives the polarization vector position. This form for the data is insensitive to the typical experimental efficiencies; any slowly varying functions are cancelled by $I_{0}(E)$.

Similarly if the theory is asked only to reproduce $x(E)$, we can concentrate on only the rapidly varying parts of the partial cross section. Specifically we may ignore various constants, density of states, and--at least for $S(1 \mathrm{~s})--$ any atomic cross-section dependence on 
energy. To simulate the measurement then we must calculate the probability that an electron will enter our angle-resolving aperture, $\Omega$, given that we are irradiating our sample with photons whose energy is 100-600 eV above the $S(13)$ absorption edge. Thus we need the continuum orbital, $\psi(\vec{r})$, from a stationary state of the light plus sample system so that we may calculate

$\hat{I}(E) \propto \int_{\Omega} \psi^{*}(\vec{R}) \psi(\vec{R}) d \Omega$.

To form the estimated $x(E)$ we should fit this $\hat{I}(E)$ with a function like that used for $I_{0}(E)$ in the experiment to give $\hat{I}_{0}(E)$.

The complete calculation of $\psi$ is a complex problem, piimarily because photoabsorption is a dynamic process. Our procedure--implicit in previous work--is to split the complete problem into two parts. The first part of the problem, which we will call the zero-order problem, contains all of the dynamical (time dependent) physics; the second part, the scattering problem, includes the scattering from ion cores as a perturbation on the zero order wave function. Thus $\psi(\vec{r})$ is constructed in the scattering problem as

$\psi(\vec{r})=\psi_{0}(\vec{r})+\psi_{1}(\vec{r})+\psi_{2}(\vec{r})+\cdots$

using $\psi_{0}(\vec{r})$ from the zero-order problem. For the remainder of this section we define these problems and discuss their connection. The detail of our discussion should not be taken to Indicate the signiflcance of these models; we present the detail to explore the basis 
of the theory and in particular to avoid subtle inconsistencies between quantum and classical ideas.

\section{A. The Zero-Order Problem}

The zero-order problem consists of the photoabsorption in the absence of ion-core scattering. To be useful for structure determination, the photoelectron continuum orbital must be representable as a wave about a single center; we are only interested in photoabsorption from localized core level initial state. Thus the zeroorder problem is nothing more than atomic photoabsorption in the presence of a surface. We imbed an atom in a tenuous medium representing the properties of the material surrounding the photoemitter absent the properties included in the scattering problem. The specific medium will then depend on what material properties we include in the scattering problem. At least we shall want the medium to represent the valence charge density in the metal which is responsible for screening the photo-ion since screening is a dynamical process. Typically, we would also ask the medium to simulate the interstitial regions of the crystal (between ion cores) smoothly continued over the whole crystal. The only important requirement for the medium is that it present only a very smooth potential incapable of scattering or attenuating the photoelectron wave anisotropically. This same property facilitates the application of classical physics to the zero-order problem. A jellium metal has the appropriate properties for the $\mathrm{S} / \mathrm{Ni}$ problem and to be specific we adopt such a model here.

One exception to the smooth potential restraint would be the imageforce (surface barrier) potential whose effects can also be treated seni-classically (see section $V$ ). 
B. Connection to the Scattering Problem.

Solution of the zero-order problem and connection to the scattering problem requires switching between quantum and classical descriptions for the photoelectron. It does not seem helpful to construct a current density ${ }^{19}$ from $\psi_{0}(\vec{r})$ since this would necessarily involve a time average while the classical currents are time-dependent. More than elementary considerations would be necessary to form wave packets to make the connection: at the time of photoabsorption the photcelectron is prepared in a state of definite angular momentum. Two sensible prescriptions are to interpret the zero-order wavefunction $\psi_{0}(\vec{r})$ as a probability amplitude for the classical electron or to construct a potential which reproduces the classical effects and apply it to the quantum problem; this is the optical potential or complex inner potential method. 20

For either the probability or optical potential connections, the dynamics of the zero-order problem enters through the kinematics of the classical electron: position and time are always related by the constant velocity. The classical electron and a core hole created very quickly $\left(10^{-20} \mathrm{sec}\right)$ in a small region near the photoabsorbing atom's nucleus with the electron's initial direction selected from a probability distribution given by the dipole selection rule and the initial state. As the electron moves away from the hole, the medium responds to the newly created charges; this is the problem addressed by Noguera et al. ${ }^{19}$ From the response we can construct the zero-order probability amplitude $\psi_{0}(\vec{r})$.

What of the effect of the medium on the scattered wavefield? Once we have passed the time required to screen the hole and photoelectron 
and for the photoelectron to separate from the hole by the sum of their screening radii, the effects of the medium will be the same whether the electron has been scattered or not. If we assume that the electron velocity is slow enough to insure that the radius to which this time corresponds does not overlap the scattering potential, then we can say that the full optical potential operates for all of the scattered waves. Alternatively we can convert the scattered wave probability amplitudes into electron trajectory probability distributions, but this would be unnecessarily complex. It is only important to recognize that the classical electron either scatters or does not scatter; the scattered classical electron feels no field from an unscattered electron current density .

\section{The Scattering Problem.}

The physical model for the scattering problem consists of a lattice of non-overlapping ion core potentials centered on the instantaneous atomic coordinates of the sample. As we discuss in more detail in the following section, the result of the zero-order problem is a screened spherical wave attenuated by the inelastic scattering properties of the conduction electrons (imaginary part of the optical potential). This zero-order wavefunction is input to the scattering problem where the ion-core potentials are introduced. Ideally these potentials would describe both the ion-core potential operating on the electron and the anisotropic contributions to the inelastic scattering. The effective potential should include neither the constant inner potential (real part of the optical potential) nor the inelastic scattering from conduction electrons (imaginary part of the optical potential) already contained in the zero-order wavefunction. Also ideally, this potential should 
describe the interaction of an electron and an ion-core embedded in the medium used for the zero-order problem. This differs from the atomic physics potential ${ }^{21}$ in two respects. First, the electron wave amplitude decays across the active region of ine potential due to interaction with the conduction electron density. Second, the atom is partially screened from the photoelectron by the conduction electron response. Since the screening length is comparable to the diameter of the potential and since the fast electron rushes ahead of its screening charge density ${ }^{22}$ the impact of this difference should be minimal for the real part of the ion-core potential. This screening should however reduce the large impact parameter inelastic excitations caused by the long range Coulomb field of the electron. These excitations affect the real part of the atomic potential through shadow scattering; ${ }^{23}$ we should expect no shadow scattering in the solid state.

To summarize, we must solve two connected problems. The zero-order problem is atomic photoemission in the presence of a jellium surface; the scattering problem incorporates the ion-core potentials via a perturbation series. The zero-order problem contains all of the time dependence; the scattering problem seeks a stationary state. The dynamics of the zero-order problem are transmitted to the scattering problem by the kinematic connection between position and time, valid for the zero-order problem. 


\section{THE ZERO-ORDER PROBLEM}

Now consider the solution of the zero-order problem. To be concrete consider a sulfur atom partially embedded in a jellium surface being irradiated by soft $x$-rays of energy above the $s(1 \mathrm{~s})$ threshold. The electron density of the jellium is chosen to reproduce the dielectric properties of $\mathrm{Ni}$ as far as this is possible. Our task is to deduce the probability amplitude $\psi_{0}(\vec{r})$ for a photoelectron ejected from the $s(1 s)$ orbital.

Atomic photoabsorption is well understood ${ }^{24}$ and as the jellium surface has only a very small interaction with the $S$ core, we need only summarize the assumptions and conclusions of the photoemission theory. For absorption, the radiation field may be treated classically; ${ }^{23}$ far from the surface it is characterized by a vector potential

$\vec{A}(\vec{r})=A_{0} E e^{-i \vec{\gamma} \cdot \vec{r}}$

This is a plane, monochromatic wave with propagation direction $\vec{\gamma}$ and polarization direction $\bar{\varepsilon}$. We need only consider polarized light since the results for partially polarized or unpolarized light may be obtained by combining two plane polarization calculations without regard to phase.

In principle, the vector potential in the region of the sulfur atom must be recalculated to include the effect of the dielectric properties of the NI surface on the incident electric fleld. Since we are solely interested in photoelectrons with intermediate energies, we may restrict our attention to photon energies greater than say $50 \mathrm{eV}$, allowing the 
index of refraction of the medium to be written as its high frequency limit:

$n(w)-1-\frac{E_{p}^{2}}{2 E^{2}}$

where $E_{p}$ is the photon energy corresponding to the medium's plasma frequency. 25 since $E_{p}-10 \mathrm{eV}$ and the lowest photon energy of interest here is $50 \mathrm{eV}$, the index will be within $2 \%$ of 1.0 as a worst case: the electric field may be considered unaffected by the surface unless we choose grazing incidence. For this exception we must consider refraction of the soft $x$-rays at the surface-vacuum interface with a consequent slight shift in the direction of polarization. 26 we may also disregard the attenuation of the photon beam which only occurs over hundreds of atomic distances. 27

The intensity of the $x$-ray beam is small enough to calculate its effect on the ground state of the zero-order model with perturbation theory, using 28

$H:=\vec{A} \cdot \vec{\nabla}$

(as noted in the introduction we may ignore all constant factors.) Among all transitions which this operator may induce, we are only interested in those whose final state may be written as a product of a photolon wavefunction with a (1s) core hole, $\Phi_{f}$, and a continuum photoelectron wave, $\Psi_{0}$, whose energy is given by the difference between the photon energy and the (1s) binding energy. Since the perturbation 
is a one electron operator, the transition amplitude to $\psi_{0}$ may be written

$\psi_{0}(\vec{r})=\left\langle r \mid \psi_{0}\right\rangle M_{1}\left\langle\Phi_{f} \mid \Phi_{0}\right\rangle\left\langle\psi_{0}|\vec{A} \cdot \vec{\nabla}| \phi_{1 s}\right\rangle$

where $\phi_{1 s}$ is the (1s) orbital and $\Phi_{0}$ is the ground state wave function excluding $\phi_{1 s^{\circ}}$. (We have grouped various constants given by standard texts $^{23}$ into $M_{1}$; they are not relevant to the oscillations we wish to calculate).

We may use the dipole approximation for this matrix element as we now demoristrate. The (1s) orbital is reasonably described by a scaled hydrogenic orbital 28

$\phi_{1 s}=\frac{e^{-z r / a_{0}}}{(\pi)^{1 / 2}}\left(\frac{z}{a_{0}}\right)^{3 / 2}$

with a binding energy near

$E_{1 s}=+\frac{z^{2} e^{2}}{2 a_{0}}$

where $a_{0}$ is the Bohr orbit radius and $z$ is the atomic charge. For ARPEFS we use electrons with kinetic energies from 50-1000 eV meaning that our photon energy will lie between $E_{1 \mathrm{~s}}+50 \mathrm{eV}$ and $E_{1 \mathrm{~s}}+1000 \mathrm{eV}$. Writing these energies in atomic units and converting to wave number we find that, over the range of the (1s) orbital, $2 a_{0} / Z$, the magnitude of the vector potential exponent in eqn. (1) will be 
$\frac{2|\gamma| a_{0}}{Z} \leq Z \alpha$

for $Z>8$ at all energies, and

$\frac{2|\gamma| a_{0}}{2} \leq \frac{70}{Z}$

for $z<8$ and $E=E_{13}+1000 \mathrm{eV}$, where $a=e^{2} / \mathrm{hc}=1 / 137$. Thus, only for the heaviest and very lightest elements (at high electron kinetic energy) will the exponent differ substantially from 1.0: for the calculation of the transition matrix element we may replace the oscillating dependence of the electric field by a constant. Further the connection between matrix elements of the momentum operator and those of the position operator then gives the dipole approximation ${ }^{28}$

$\frac{\partial \sigma}{\partial \Omega}=M_{2}\left\langle\Phi_{f} \mid \Phi_{0}\right\rangle\left\langle\psi_{0}|\hat{\varepsilon} \cdot \vec{r}| \phi_{1 s}\right\rangle$

We have neglected both exchange and relativistic corrections, but these effects are no larger than the errors of the dipole approximation. The exchange corrections are small for the zero-order problem since the photoelectron wavenumber is much larger than the conduction electron wavenumber. Simllarly, the electron velocities in the zero-order problem are always small and hence the relativistic correction may be ignored.

To evaluate the dipole matrix element, we expand $\psi_{0}(\vec{r})$ in eigenfunctions of the core-hole potential problem. Without specifying 
their form we may take these eigenfunctions as separated in spherical coordinates:

$\psi_{0}(\vec{r})=\sum_{n \ell m} A_{n \ell m} R_{n \ell m}(r) Y_{\ell m}(\theta, \phi)$

Since $\vec{\varepsilon} \cdot \vec{r}=r \cos \theta$, the angular integration of the dipole matrix element requires $\ell=1.23$ Then

$\Psi_{0}(\vec{r})=M_{3}\left\langle\phi_{f}\right| \Phi_{0}>R_{100}(r) Y_{10}(\theta, \phi) \int_{0}^{\infty} R_{100}(r) e^{-Z r / a} r^{3} d r$.

We have proceeded to this point as if we were calculating a stationary state despite our original claim that the zero-order problem was dynamic. We can now justify and remedy this flaw by invoking the work of Noguera et al ${ }^{19}$ on dynamic core-hole screening. They consider the entirely classical problem of the response of a jellium metal to the Instantaneous creation of a core hole and photoelectron. They demonstrate that the medium cannot respond instantly to the photoabsorption event, and therefore the screening of the core hole and photoelectron may be neglected for a short period of time following the photoabsorption. If the electron is traveling fast enough, it will leave the region of the core hole before the medium can modify the ion core field. Thus, for the sake of the zero-order problem, and over the region in space which contributes to the dipole matrix element, we may ignore the medium in calculating the transition matrix element, as we have done above. 
To this point we have determined the angular dependence of the zero-order wave function. To proceed it would seem that we must specify $R_{100}(r)$ and solve the dynamic problem of core-hole screening. Fortunately, this is not necessary as long as we only ask the theory to produce the form of the ARPEFS. Using the connection between position and time, we may divide the radial distance from the photoabsorber nucleus into three regions. We have discussed the first region above, the Inner core region where the screening properties of the surface are negligible. The second region extends from the inner core region to the point at which the core-hole potentlal is fully screened; this point depends on the photoelectron velocity, $v$, and the response time of the material, $\tau$ :

$r_{\rho}=v \tau$

Throughout the third region, beyond $r_{f}$, the dynamics are again Irrelevant--the electron and hole are screened--and we have a free spherical wave in this region.

If $r_{f}$ is less than the atomic radius, $r_{a}$, of the photoabsorber then the core hole may be said to be screened before the scattering problem, and we need only the form of the scattered wave in the third region; it must be a spherical wave traveling in the optical potential of our zeroorder medium. Thus

$\Psi_{0}(\vec{r})=A\left(r_{f}\right) e^{-\left(r-r_{a}\right) / 2 \lambda} 1 h_{1}(k r) Y_{10}(\theta, \phi)$ 
$A\left(r_{f}\right)=|A| e^{i \delta_{1}(k)}$

and the optical potential gives a mean free path $2 \lambda$, and an energy shift, $k_{f}^{2}=k^{2}+E_{0}$. All the details of absorption cross section, core relaxation, dynamic screening, and so on, serve only to determine the amplitude $|A|$ and $\delta$, phase of the spherical Hankel function. Furthermore, this amplitude and phase are independent of emission direction so that the direct wave and all orders of scattered waves contain the same $A\left(r_{a}\right)$. When the ARPEFS is calculated, both the amplitude and phase of $A\left(r_{a}\right)$ cancel: the details of photoabsorption of $r<r_{a}$ are irrelevant for the oscillations since $r_{f}$ does not overlap any scattering atoms. Note that the mean free path of the probability amplitude is twice the mean free path of the electron current density. If $r_{p}>r_{a}$ then the zero-order wave will still feel a partial Coulomb potential from the core hole, and its form must lie between that of a Coulomb wave and spherical Hankel function. To estimate $r_{f}$ we refer then to the work of Noguera, et al. 19 These authors argue that the screening time is a fraction of the plasmon period, $\left(2 \pi / \omega_{p}\right)$, of the medium; for the real part of the dynamic potential they use

$r_{f}=0.15\left(\frac{2 \pi}{\omega_{p}}\right) v=0.15 \frac{h^{2} k}{m E_{p}}=1.3 \frac{k}{E_{p}}$

Where $v$ is the electron velocity, $k$ is its wavenumber in $A^{-1}$ and $E_{p}$ is the plasmon energy in electron volts. The photoelectron velocity at $k=5 \AA^{-i}$ is $58 \mathrm{~A} / \mathrm{femtosec}$ and at $k=10 \mathrm{~A}^{-1}$. It is $139 \mathrm{~A} / \mathrm{femtosec}$. For a typical metal $E_{p}-13 \mathrm{eV}$, and for the range of $k$ between $5-12 A^{-1}$ we have 
$r_{f}$ from 0.5 to $1.2 A^{-1}$. Thus the use of screened waves seems justified for metalg although the validity of this criterion should be verified experimentally.

For semiconductors or insulators we should expect $r_{f}>r_{a}$ and some correction for dynamic effects may be required. The most important dynanic corrections would adjust the phases and amplitude of $\psi_{0}(\vec{r})$ in the center of the region of the surrounding scattering atom to account For partial screening, neglecting the variation of the phase and amplitude across the potential volume. Noguera and Spanjaard 29 nave developed such a correction for EXAFS; the EXAFS central atom phase shift is precisely the phase shift of $\psi_{0}(\vec{r})$ we seek. For the amplitude correction, the results of Noguera and Spanjaard do not seem to apply. If we make a crude approximation that the imaginary part of the optical potential rises to its asymptotic value linearly from zero in a time which is a constant of the material (fraction of a plasmon period):

$$
\begin{array}{rlrl}
V_{I}(r) & =V_{I}(\infty)\left(r / r_{f}\right) & r & \leq r_{f} \\
& =V_{I}(\infty) & r>r_{f}
\end{array}
$$

Then the effective optical potential attenuation will be:

$$
\begin{array}{ll}
\exp \left[\frac{V_{I}(\infty)}{h v} \frac{r}{2}\right] & r \leq r_{r} \\
\exp \left[\frac{V_{I}(\infty)}{h v}\left(\frac{r-r}{2}\right)\right] & r>r_{r}
\end{array}
$$


where the mean free path $\lambda=h v / V_{I}(\infty)$. Thus the delayed response of the material reduces the apparent distance of attenuation through the medium. Such a correction would have its major impact on nearest neighbors where $\left(r_{f} / 2\right)$ is a larger fraction of the internuclear distance; we cannot explain the opposite behavior claimed by Noguera and Spanjaard. Until this issue is further clarified, we will restrict our attention to metals.

The development of the theory of EXAFS has lead to the introduction of a similar type of attenuation correction. In a preliminary EXAFS theory Lee and Pendry used a static mean free path attenuation of $\exp \left(-2 r_{j} / \lambda\right)$ where $2 r_{j}$ is the path length traveled by the EXAFS electron in returning from atom $j$. Stern, Bunker, and Heald have proposed to replace this attenuation by

$s_{0}^{2}(k) e^{-2\left(r_{j}-\Delta\right) / \lambda}$

The factor $s_{0}^{2}(k)$ is empirically $y^{30}$ and theoretlcally $y^{31}$ found to be a constant; it is given by a weighted sum of overlaps between "passive" electron wavefunctions in the absorption cross-section calculation. If these wavefunctions contain the valence electrons, this overlap correction should account for inelastic losses at the central atom (photoabsorber); hence an atomic radius, $\Delta$, must be removed from the path length in the attenuation factor. Identifying $2 \Delta$ as $\left(r_{f} / 2\right)$ connects this attenuation factor and our version of the dynamical response of a classical medium given above. In relating this attenuation factor to ARPEFS we not 1 ce that photoemission measures only one channel in the absorption cross section so that $\mathrm{s}_{0}(k)$ consists of a 
single term (in our notation $S_{0}(k)=\left\langle\Phi_{\mathrm{f}} \mid \Phi_{0}\right\rangle$ ) and canceis when the oscillations are formed.

We deduce from all of these considerations that our zero-order wavefunction will be

$\Psi_{0}(\vec{r})=|A| e^{i \delta\left(r_{d s}, k\right)} i h_{1}(k r) Y_{10}(\theta, \phi) e^{-r_{d s} / \lambda}$

where

$r_{d s}=r / 2$ if $r \leq r_{f}$
$r_{d s}=r-\left(r_{f} / 2\right)$ if $r>r_{f}$.

In reviewing our procedure to this point notice in particular that

1) the angular distribution of $\Psi_{0}$ is determined by the dipole selection rules,

11) we assume that the core hole is "mostly" screened, including dynamic effects only via a modified optical potential, and

111) all constant or slowly varying factors ultimately cancel.

On this last point we note that the total cross section may sontain some structure at the lower end of the ARPEFS energy range.

We have thus $f$ ar ignored the surface in our formulation of the zero-order wavefunction. It is evident that the semiclassical methods $^{19}$ can be applied to understand the required changes in $\Psi_{0}(\vec{r})$ when the photoabsorber is near, at, or above the jellium surface. 32 Lacking such a study we can anticipate some of the conclusions as follows. Consider first the static case, long after the photoelectron 
has left the photoion. If the ion lies below the surface by more than the Debye screening length, $\lambda_{D}$, we may ignore the surface. If on the other hand the ion lies above the surface by more than $\lambda_{D}$, then the screening must be accomplished by a surface charge density: an image charge is formed by rearranging electron density in the surface region below the ion. For the dynamics of the core-hole screening of an atom above the surface the relevant time scale would seen to be a fraction of the surface plasmon period, $\left(2 \pi / \omega_{3}\right)$. For the simplest model of plasmons, $\omega_{s}=\omega_{p} /(2) !^{1 / 2}$ and the period, and hence the screening time would be $40 \%$ longer. If the ion lies within $\lambda_{D}$ of the surface, the surface and bulk plasmons will share the duty of neutralizing the core hole, but the division of labor will depend on their relative densities.

For the dynamics of the photoelectron screening, we can distinguish those electrons entering the material from those emitted into the vacuum. As long as the photoabsorbing atom is within an atomic radius of the surface, we would expect the photoelectron entering the material to react as if the surface did not exist. As the electron travels from the core hole to the jellium, the medium--be it all jellium or partly vacuum--has not enough time to respond; by the time the medium has recovered, the photoelectron has penetrated the metal. For a photoelectron ejected into the vacuum, however, there is no bulk charge density to consider. The response of the screening surface charges to the suddenly created core hole will be similar to its response to a scattered photoelectron suddenly emerging from the surface: we will treat this response in section $V$.

With only these very qualitative ideas to proceed with, it is difficult to be very certain of the modification of the zero-order 
wavefunction for a photoemitter near the surface. For electrons emitted into the material, we only have the more slowly screened hole to confront; we expect the full screening radius to be $1.0-1.4$ times larger than it would have been without the surface. Thus for $\vec{r}$ below the surface we retain equation (2) with $r_{f}$ of selected somewhat larger. For electrons ejected into the vacuum, the attenuation due to electronelectron scattering stops at $\mathrm{r}_{\mathrm{a}}$ and the photoelectron travels away from a potential which for short times and distances is a bare core hole while for larger times and distancés, it is a small dipole created by the core hole and its image charge. (The charge of the dipole is given by the integral of the surface fraction of the screening charge). At even larger times, on the order of $(2 \pi / \Delta E)$ where $\Delta E$ is the photopeain lifetime width, the potential of the core hole decays to zero. Due to this potential, the wavefunction in the vacuum region will experience a stronger phase shift than the wave in the region occupied by jellium. Thus for $\vec{r}$ above the surface we select $r_{d s}=r_{a}$ for attenuation and add some phase shift due to the dipole in $\delta\left(r_{d s}, k\right)$.

We have constructed an elaborate physical description of the zeroorder problem, and guided by the not very specific model calculations of Noguera, reduced the dynamics of the zero-order problem to a single number, $r_{f}(k)$ and an unknown dipole phase shift. Certainly our justification for our result is the lack of alternative treatments; we can, however, produce a convincing argument that the major dynamics effects are included and furthermore if we are forced to ignore the dipole correction for now, the errors will be small. Our argument has two parts. First, we cite the extensive 11 terature of EXAFS and in particular the recent theoretical study ${ }^{33}$ of Pt EXAFS in which dynamic 
This report was done with support from the Department of Energy. Any conclusions or opinions expressed in this report represent solely those of the author(s) and not necessarily thase of The Regents of the University of California, the Lawrence Berketey Laboratory or the Department of Energy.

Reference to a company or product name does not imply approval or recommendation of the product by the University of Califomin or the U.S. Department of Energy to the exclusion of others that may be suilable. 


\section{THE SCATTERING PROBLEM}

Having assigned all of the dynamics of photoemission to the zeroorder problem and assuming that we have a satisfactory solution in the form of a zero-order wavefunction, we face a straightforward electron scattering problem to introduce the effect of the ion cores. We will persist in the convenient terminology of describing calculation of an additional ion core at any scattering order as a scattering of the electron from the ion-core potential. Because of the kinematic connection between position and time, this terminology will be a useful guide to physics within a given order, but it can lead to difficulty if applied across orders. For example, the third of three colinear atoms is not shadowed from the first in the single scattering calculation; the shadow only appears when forward scattering from the central atom scatters from the third atom during the second order or double scattering calcliation.

Our array of spherically symmetric ion cores is the familiar muffin-tin model for solids and the scattering problem itself has been extensively investigated. While Tong and coworkers ${ }^{4}$ have applied the multiple scattering methods of LEED directly to the scattering problem we face, the usual techniques become unmanageable in the higher energy range. We have investigated the difficulty posed by the higher energy range, and we have proposed a new approximate method for intermediate energy range. 17 This method, which we will call TS-MQNE: Taylor seriesmagnetic quantum number expansion, is fully developed in ref. 17 Therefore we will concentrate here on the form of this approximate method and its application to ARPEFS. 
The MQNE may be qualitatively described as follows. The basic ingredients in the scattering problem is a wave source, a scattering potential, and an observation point. The wave source may be the original photoemitting atom, or a scattering atom. The scattering potential is taken to be spherically symmetric. The observation point may be a detector or another scattering potential. The first step in the MQNE is to describe the basic scattering problem in a coordinate system in which the $\hat{z}$ axis lies along the vector between the wave source and the scattering potential. Thus we decompose the source wave into partial waves of orbital and magnetic angular momenta quantized along the internuclear axis between source and scattering atoms. If the source wave is originally described in terms of partial waves about a different axis, then this step introduces rotation matrices for the spherical harmonics.

The second step of the MQNE procedure translate the source partial waves to the scattering potential. This translation conserves the magnetic quantum number--the first of two reasons we choose the internuclear axis for the quantization axis. The third step of the MQNE procedure generates the outgoing scattered wave by multiplying each Individual incoming partial wave by a complex scattering matrix element. Our convenient choice of the internuclear separation as the quantization axis now helps a second time: only waves with low magnetic quantum numbers scatter from the potential. The number of waves is directly related to the ratio of the potential radius to the internuclear separation. The restriction of the magnetic quantum number scattered by the potential constitutes the MQNE. 
We can understand the physical basis of the MQNE, either pictorally ${ }^{17}$ or from integral equations for the scattered wave, as a consequence of the finite range of the potential. The source wave spherical harmonics, when quantized along the internuclear axis, have lobes which peak further and further from the axis as the magnetic quantum number increases. Thus for some magnetic quantum number, the corresponding source wave component does not overlap the potential at all, and it may be ignored in calculating the scattered wave. A similar approximation to the radial character of the source wave based on a Taylor series approximation is described in ref 17 . Together these approximations give the TS-MQNE method.

The zero-order wavefunction is an $\ell=1$ spherical wave centered on the photoabsorbing atom: at our detector, $\vec{R}$, the wave is

$\psi_{0}(\vec{r})=\frac{e^{i k R}}{1 k R} \cos \theta_{E R} e^{-L(0) / 2 \lambda}$

where the electric vector $\varepsilon$ acts as the $\hat{z}$ axis and the photoabsorber is the origin. The factor $L(0)$ is the distance from the photoemitter to the surface barrier in the direction of the detector. The first order wavefunction has spherical waves emanating from every ion core except the photoabsorbing atom:

$\Psi_{1}(\vec{r})=\sum_{a_{j \neq 0}} \frac{e^{i k\left|\vec{R}-a_{j}\right|}}{i k\left|\vec{R}-\vec{a}_{j}\right|} \frac{e^{1 k a_{j}}}{a_{j}} F\left(\vec{\varepsilon}, \vec{a}_{j}, \vec{R}\right) e^{-a_{j} / 2 \lambda} e^{-L\left(a_{j}\right) / 2 \lambda}$.

The index $j$ runs over all near by atoms. We may expand 
$\left|R-a_{j}\right|=R\left(1-\frac{a_{j}}{R} \cos \theta_{a_{j} R}+\cdots\right)=R-a_{j} \cos \theta_{a_{j} R}$

to write

$\frac{e^{i k\left|\vec{R}-\vec{a}_{j}\right|}}{i k\left|\vec{R}-\vec{a}_{j}\right|}=\frac{e^{i k R}}{i k R} e^{-i k a_{j} \cos \theta_{a_{j} R}}$

The second and third term in the first order wavefunction accounts for the phase and amplitude of the zero-order wave at the center of the scattering potentiai at $\vec{a}_{j}$. The complex number $F\left(\vec{\varepsilon}_{,} \vec{a}_{j}, \vec{R}\right)$ is the scattering factor and it gives the phase and amplitude of the scattered wave in the direction of the detector; its precise nature depends on the scattering approximation chosen. In a plane wave model, $F(\vec{\varepsilon}, \vec{a}, \vec{k})$ would be the scattering factor of atomic physics times $\cos \theta_{\epsilon a}$. The final term in the first order wavefunction is the attenuation of the scattered waves as they propagate from the scattering atom to the surface, a distance of $L\left(\vec{a}_{j}\right)$.

Similarly, the double scattered wave is

$\psi_{2}(R)=\sum_{j \neq 0} \sum_{a_{j} \neq 0} \frac{e^{i k R}}{i k R} e^{-1 k a_{j} \cos \theta_{a_{j} R}} \frac{e^{i k a_{j}+a_{j} / 2 \lambda}}{a_{j}}$

$$
F\left(\vec{\varepsilon}, \vec{a}_{j}, \vec{b}\right) e^{i k a_{j} \cos \theta_{a_{j} R}} \frac{e^{i k b_{j}+b_{j} / 2 \lambda}}{b_{j}} F(\vec{\varepsilon}, \vec{b}, \vec{R}) e^{-L\left(\vec{a}_{j}+\vec{b}_{j}\right) / 2 \lambda} .
$$

and the higher order waves may be written down by analogy. 
Interference between the zero-order wave at the detector--which we will call the direct wave--and the single and double scattered waves occurs when the complete wave probability is calculated:

$$
\begin{gathered}
\psi(\vec{R}) \psi(\vec{R})=\psi_{0}^{*} \psi_{0}+\left(\psi_{0}^{*} \psi_{1}+\psi_{1}^{*} \psi_{0}\right)+\left(\psi_{0}^{*} \psi_{2}+\psi_{2}^{*} \psi_{0}\right)+\psi_{1}^{*} \psi_{1} \\
\left.+\psi_{1}^{*} \psi_{2}+\psi_{2}^{*} \psi_{1}\right)+\cdots
\end{gathered}
$$

The interference leads to ARPEFS when the proportional oscillations are formed.

We detect the photoemission intensity with a small but finite angular acceptance and the scattering atoms vibrate sc ihat every photoelectron scatters from a slightly different systim. We must consider this angle integration and thermal averaging before we can hope to simulate the experimental ARPEFS. We have also neglected any effect of surface on the photoelectron. We take up these topics in the next three sections. 
V. REFRACTION AND THE INNER POTENTIAL

The optical potential introduced to represent the interaction between the fast photoelectron and the conduction electrons of the solid consists of both a real and an imaginary part. As we discussed in section III, the imaginary part of the optical potential serves to attenuate the photoelectron wave; its physical origin is the excitation of plasmon oscillations. The real part of the optical potential plays no role in scattering; it represents the energy difference between a free electron and that electron screened by the conduction electrons of the solid. When the photoelectron leaves the solid to be detected, it is no longer under the influence of the optical potential. The termination of the imaginary part of the optical potential simply ends the attenuation, a fact which is recognized in the definition of $L\left(\vec{a}_{j}\right)$ above. (We assume that the attenuation of the photoelectron due to surface plasmon excitation cancels, being identical for direct and scattering waves.) Now we consider the effects of terminating the real part of the optical potential.

We return to the model zero-order problem in ignoring the ion-core potentials. A photoelectron with energy $E$ within the jellium medium will have energy $E-E_{0}$ in the vacuum far from the surface. This loss of kinetic energy $E_{0}$ may be relaied to a potential barrier whose total helght is $V_{0}$, the real part of the optical potential. The height of the barrier determines the energy loss, but the barrier shape will alter the photoelectron's path. Sagurton et al. 13 adopted a planar step barrier of helght $v_{0}$ just outside the last row of ion cores. This is the usual first-order model for the surface barrier, introduced for both low energy photoemission ${ }^{27}$ and low-energy electron diffraction. 20 
The important consequence of this model is a prediction that the emerging photoelectron will be refracted in a direction away from the surface normal in the manner of optical refraction with

$$
(E)^{1 / 2} \sin \theta_{\text {in }}=\left(E-E_{0}\right)^{1 / 2} \sin \theta_{\text {out }}
$$

where the angles are measured from the surface normal. Since $E_{0}<E$ for ARPEFS energies, the angle correction is small, but it could be significant for high accuracy at larger emergence angles. This correction is, however, not a consequence of the photoemission physics but only results from the particular choice of the potential barrier. As we now discuss, every move to improve the description of the physical barrier serves to reduce this already small correction, to the point that we may neglect it.

To justify our claim that refraction does not apply to ARPEFS, we must return to arguments similar to those of section III. The step barrier is incorrect for three reasons. First, the surface barrier is not abrupt and planar, i.e. the conduction electron density does not drop suddenly to zero along a plane. This is true even for a jellium model whose positive jelly does terminate abruptly. ${ }^{20}$ More appropriate here, structure methods which are sensitive to the corrugation of conduction electron density, for example scanning tunneling microscopy, ${ }^{34}$ or He diffraction, 35 shows a distinctly non-planar surface. Since the refraction is already small, even a small undulation of the surface will invalidate the optical refraction formula above. This argument does not necessarily invalidate the use of a planar barrier for lower energies where the photoelectron wavelength will be 
much larger than the atomic scale corrugation of the potential and the photoelectron wave is sensitive only to the average surface potential. Second, the interaction which gives rise to the surface barrier is not proportional to the local charge density as the step barrier model would imply. A slowly moving photoelectron is surrounded by a localized cloud of low electron density created by Coulomb repulsions between the photoelectron and the conduction electrons. 22 Thus the total screening energy is related to the charge density. However, when the slow photoelectron and its co-moving cloud reach the surface, the coulomb interaction which created the cloud does not cease. Instead, the screening cloud spreads out along the surface, becoming the surface charge density which screens the Coulomb potential of the photoelectron from the bulk metal. This net positive surface charge density is equivalent to an image charge; the photoelectron must wor' a gainst the image charge attraction giving the surface barrier. ${ }^{36}$ Thus the surface barrier is not abrupt even for a planar surface but rises smoothly from a constant to an image potential, $v(z)=1 /(z+c)$, over distances comparable to the Debye screening length $\lambda_{D}(-0.5 \mathrm{~A})$.

Third, and most important, the potential is velocity dependence for the same reasons that give rise to the dymamic soreening effects described in section III. A fast electron within the bulk of the medium trails a wake of low electron density corresponding to sluggish conduction electrons moving away from the photoelectron charge. 37 When photoelectron emerges from the surface, the center of gravity of its screening charge density is some distance below the surface and the force it exerts upon the photoelectron is directed along the velocity vector of the photoelectron, not along the surface normal. 
As the photoelectron moves into the vacuum, the surface charge density is similarly slow in redistributing, leading to an image charge which always lags behind the photoelectron as it moves along and away from the surface. For normal emission, this dynamic effect serves only further stiooth the potential barrier in the region near the surface. 38,31 For off-normal emission, the lag between the position of the image charge parallel to the surface and the position of the photoelectron parallel to the surface increases with the emergence angle to normal; $1 . e$. with the velocity of the photoelectron along the surface. Thus, rather than experiencing a force along the surface normal, the photoelectron works against a force more nearly along its velocity vector. Such a force does not alter the photoelectrons path.

To summarize, we do not agree with the extension of the optical refraction model to intermediate energies proposed by Sagurton, et al. This leaves the inner potential shift as the only effect of the terminating real part of the optical potential. The forces for offnormal trajectories certainly merits more careful theoretical treatment particularly for understanding the transition between the lowest energy range where optical refraction should apply and the ARPEFS energy range where the forces are along the velocity. Furthermore, there seems to be very little experimental evidence on refraction despite its fundamental nature. 39 We should also note that our description does not contradict the classical studies of electron beam refraction: ${ }^{40}$ when dynamic potentials are considered, both the incident and refracted electron beams are modified. 41

In the following sections we will continue to use the wavevector $k$ corresponding to the electrons energy in the solid even when we are 
discussing the probability amplitude at the detector. The theory results are much more simply expressed this way so that we use the inner potential to convert the experimental kinetic energies to a wavenumber rather than shifting the theory.

We should ncte that the inner potential for ARPEFS is the same as that used in LEED ${ }^{20}$ but not the same as the $E_{0}$ used in EXAFS. The only energy relevant for the scattering problem is the electron's kinetic energy when it encounters a scattering potential. In ARPEFS and in LEED the scattered electron is detected, and the inner potential represents the physical kinetic energy lost when the electron travels from the scattering potential edge to the detector. From our previous considerations, this inner potential should be velocity dependent. The conduction electrons are slow to respond to the photoelectron so the positive charge responsible for the inner potential lies in a wake whose center of gravity lies further away from the photoelectron the higher the energy. Usually, the energy dependence is rieglected. In EXAFS, the scattered electron is not detected, and the "inner potential", $E_{0}$, is a complicated weighted sum of all the photoelectron energies created at a particular $x$-ray photon energy, ${ }^{42}$ and $f$ urther it is commonly used as an adjustable parameter.. 12 High precision surface structure determination requires $E_{0}$ to be known to within $1 \mathrm{eV}$, suggesting that $\mathrm{f}$ urther study of the energy dependence of the inner potential would be profitable. 


\section{FINITE ANGULAR RESOLUTION}

The experimental apparatus for measuring the ARPEFS oscillations has a small but finite angular resolution characterized by half the angle subtended by the aperture at the source, which we call, $\alpha$. For small apertures, $\alpha$ is the radius of the aperture projected on a unit sphere so that the detected area is $\pi \alpha^{2}$. The major effect of this finite resolution is to limit the highest observable path-length difference, $p_{j}=a_{j}\left(1-\cos \theta_{j}\right)$ such that all paths with

$k p_{j} \gg 1 / \alpha$

will be averaged away by the opening, while oscillations corresponding to path-length differences

$k p_{j} \ll 1 / \alpha$

will be individually resolved. With experimental angular resolution of $\pm 3^{\circ}$, we have $\mathrm{kp}=1 / \alpha$ : $\mathrm{kp}$ will fall between 10 and 100 in practical cases and $1 / \alpha-10$. Thus we are in the regime of partial angle averaging, and we must consider the effect in detail. Fadley ${ }^{43}$ has previously demonstrated by numerical example that angle-averaging has important effects on the theoretical calculations of azimuthal and energy-dependent photoelectron diffraction. Here we will show that

1) the primary angle-integration effect is the integration of scattering angle contributions to the path-length difference,

ii) the angle integration preferentially attenuates scattering events with angles near $90^{\circ}$, and 
1ii) the effective scattering angle can be shifted by the angle integration, if the aperture is large enough.

If we use $\vec{R}_{0}$ to denote the position of the aperture center, our angle-averaged intensity is

$\bar{I}=\int_{0}^{\alpha} \sin \theta_{\mathrm{RR}_{0}} d \theta_{\mathrm{RR}} \int_{0}^{2 \pi} d \phi_{x R_{0} R}\left(\psi_{0}+\psi_{1}+\psi_{2}\right)^{*}\left(\psi_{0}+\psi_{1}+\psi_{2}\right)$

for double scattering.

We distinguish three terms from the expansion of the squared total wave function 1 ) direct intensity, $\left.\bar{I}_{00}, 1 i\right)$ scattered-direct intensity, $\overline{\mathrm{I}}_{0 s}$, and $11 i$ ) scattered-scattered intensity, $\overline{\mathrm{I}}_{\mathrm{ss}}$

$\bar{I}=\bar{I}_{00}+\bar{I}_{0 s}+\bar{I}_{s s}$

where

$\bar{I}_{00}=\int \psi_{0}^{*} \psi_{0} d \Omega$,

$\bar{I}_{0 s}=\int \psi_{0}^{*}\left(\psi_{1}+\psi_{2}\right)+\left(\psi_{1}+\psi_{2}\right)^{*} \psi_{0} \mathrm{~d} \Omega$,

and

$\bar{I}_{s s}=\int\left(\psi_{1}+\psi_{2}\right)^{*}\left(\psi_{1}+\psi_{2}\right) d \Omega$.

Each term contains the coordinates of $\hat{R}$ in the amplitudes of the wavefunction, but the interference terms $\bar{I}_{0 s}$ and $\bar{I}_{s s}$ also contain $\hat{R}$ in the wavefunction phase. As the position of $\hat{R}$ moves around the aperture, 
the wavefunction amplitude always lies near its value at $\hat{R}_{0}$. The phase of the wavefunction is, however, quite sensitive to the position of $\hat{R}$ and even for small apertures this variation should be integrated. In Appendix A we Integrate the TS-MQNE wavefunctions by a general method which considers both amplitude and phase variations and which applies to apertures approaching $\alpha=\pi / 2$. This leaves us free to concentrate solely on the qualitatively features of the angle integration for the remainder of this section.

Let us assume small apertures and ignore the variation in wavefunction amplitude. The direct intensity integrates to the aperture area times the intensity at $R_{0}$

$\vec{I}_{00}=\psi_{0}^{*}\left(\hat{R}_{0}\right) \psi_{0}\left(\hat{R}_{0}\right) \pi a^{2}$

Each term in the direct-scattered intensity will be of the same form:

$\bar{I}_{0 s}(\vec{a})=2 R e \psi_{0}^{*}\left(\hat{R}_{0}\right) \psi_{a}\left(\hat{R}_{0}\right) \int e^{-i k \vec{a}\left(\hat{R}-\hat{R}_{0}\right)} d \Omega$

where $\vec{a}$ must represent the bond vector for the scattering event which immediately precedes the trip to the aperture. For small apertures we show in Appendix A that

$\int_{0}^{a} \sin \theta d \theta \int_{0}^{2 \pi} d \phi e^{-i k \vec{a}\left(\hat{R}-\hat{R}_{0}\right)}=\pi a^{2}\left[\frac{2 J_{1}\left(k a \alpha \sin \theta_{a R_{0}}\right)}{k a \alpha \sin \theta_{a R_{0}}}\right]$ 
where $J_{1}(x)$ is the first order Bessel function. The factor of $2 J_{1}(x) / x$ is the circular, two-dimensional analog of sin $x / x$ familiar from diffraction phenomenon; ${ }^{44}$ its value at $x=0$ is 1.0 , and it falls to zero at $x=3.8$, continuing to higher $x$ with ever smaller oscillations (see figure 1).

By relating the physical parameters to the argument of the aperture damping function, i.e. $x=\alpha k a \sin \theta_{a R_{0}}$, we draw the following conclusions:

1) backscattering and forward-scattering atoms are not significantly attenuated by angle integration because $\sin \theta-$ 0 ,

1i) side-scattering $\left(\theta-90^{\circ}\right.$ ) atoms are maximally attenuated (sin $\theta-1)$.

1ii) the attenuation increases with bond length, aperture radius, and the square root of the energy.

In Fig. 1, we plot the aperture damping function for $\alpha=3^{\circ}$ and $\alpha=10^{\circ}$. We also mark the range of ka expected for side-scattering ARPEFS from nearest neighbor atoms $(a=2.2 A)$ and more distant atoms $(a=7.5 A)$. Both apertures attenuate the more distance atoms; the wider aperture even damps the nearest neighbor side-scattering atoms. We may further conclude that

iv) as long as single scattering dominates, apertures of $=10^{\circ}$ would simplify the ARPEFS spectra by eliminating sidescattering atoms.

Since the larger aperture would decrease the data collection time by an order of magnitude this conclusion merits further discussion. A sidescattered wave which subsequently forward scatters through a small angle 
into the detector will not be attenuated. The larger aperture will also introduce highe: terms into the phase integral, eqn. (3), and require treatment of the amplitude variation. In Appendix A we show that the second order term in the phase integral introduces a phase shift of $\alpha^{2} k a \cos \theta$. This correction is largest for back- and forwardscattering, and amounts to $1.5 \%$ of the bord length for an aperture of $10^{\circ}$. This larger aperture would also require calculation of the amplitude integration terms as indicated in Appendix A.

Finally, we note that the scattered-scattered intensity may be treated in the same fashion as the direct-scattered term by replacing the last scattering event bond vector, $\vec{a}$ above, by the vector difference in the last scattering bond vectors from the two interfering scattered paths in the scattered-scattered case. In most experimental geometries $\left|\Psi_{0}\right| \gg\left|\Psi_{g}\right|$ so that we may neglect $\overline{\mathrm{I}}_{\mathrm{sg}}$ altogether. In these cases, it is convenient for numerical calculations to associate the angle attenuation with the scattered waves. By this means a single attenuation factor is required for each path rather than a factor for every possible pair of paths. 


\section{THERMAL AVERAGING}

As the final ingredient in our theory we must allow for the thermally excited vibrational motion of the atoms in the surface. Each atom in the crystal oscillates about some equilibrium position; each photoemission event takes a snapshot picture of the instantaneous positions of these atoms. The complete ARPEFS spectrum averages over many photoemission events and hence records an average of the atomic positions according to the probability of each configuration. For typical materials at typical temperatures, the amplitude of the oscillations is not large. However, the effect on the ARPEFS spectrum is significant both in magnitude--thermal averaging is primarily responsible for limiting the highest measurable energy--and in detail-inappropriate thermal averaging can lead to theoretical calculations with far too many scattering events contributing (compare Bullock ${ }^{11}$ to Sagurton ${ }^{13}$ ). Our problem is formally similar to $x$-ray diffraction where thermal averaging leads to multiplication of diffracted intensity by a Debye-Waller factor, $\exp \left(-2|\vec{K}|^{2} \sigma^{2}\right.$ where $\vec{K}$ is the momentum charge in scattering and $\sigma^{2}$ is the mean square displacement projected on $\vec{k} .45$ Since the momentum change in ARPEFS may be written

$\vec{K}_{a R}=k(\hat{R}-\hat{a})$

for single scattering off an atom at $\vec{a}$ into a detector at $\vec{R}$, Bullock et al ${ }^{11}$ introduced a factor

$e^{-2|\vec{k}|^{2} \sigma^{2}}=e^{-k^{2}(1-\cos e) \sigma^{2}}$ 
where $\cos \theta=\hat{a} \cdot \hat{\mathbf{R}}$ to introduce thermal averaging to electron scattering. However, as has been described for the more analogous problem of thermally averaged EXAFS, ${ }^{46}$ the form of the $x$-ray diffraction result is adequate only if the mean square displacements are replaced by the mean square relative displacements. This difference is crucial for electron scattering: the motions of near neighbor atoms are correlated so that the mean square relative displacements of near neighbor is much less than their mean square displacements.

Incorporating the displacement correlations, Sagurton, et al 13 found considerable improvement in the agreement between theory and experiment for $\mathrm{S}(1 \mathrm{~s})$ ARPEFS from $\mathrm{S} / \mathrm{Ni}(100)$ over that reported by Bullock. In fact, these authors demonstrate that no thermal averaging at all produces a more reliable result than eqn. (4) with mean square displacements.

The thermal average, like the aperture integration discussed in the previous section, must be performed on the intensity oscillations; we will restrict our attention to experimental geometries which emphasize direct wave interference and ignore the scattered-scattered interference. Furthermore, to avoid obscuring the thermal average with the MQNE notation, we will only consider averaging the zero-order Taylor series term. This is not a serious restriction: the variation in the scattering amplitude over the range of typical vibrational motions is small, and we may safely ignore the variation in the corrections to scattering amplitude. An advantage of this approach may be appreciated by examining ref. 17, App. A and ref. 16: the thermal average will lead to derivatives of the zero-order scattering factors with respect to internuclear coordinates times displacements of the coordinates, and we 
have already encountered the derivatives in the study of spherical wave corrections.

Writing angle brackets for the operation of thermal ensemble averaging, we need for single scattering

$x_{1}=\left\langle 2 R e \frac{\cos \theta_{\varepsilon a}}{\cos \theta_{E R}} \frac{\mathrm{f}_{a R}^{00}}{a} e^{-(a-L(\overrightarrow{0})) / 2 \lambda} e^{-L(\vec{a}) / 2 \lambda} e^{i k a\left(1-\cos \theta_{a R}\right)}\right\rangle$

and for double scattering

$$
\begin{aligned}
& x_{2}=\left\langle2 R e \left[\frac{\cos \theta_{\varepsilon a}}{\cos \theta_{\varepsilon R}} \frac{f_{a b}^{00}}{a} e^{-(a-L(\overrightarrow{0})) / \lambda} e^{\left.i k a, 1-\cos \theta_{a b}\right)}\right.\right. \\
& \left.\left.\frac{f_{b R}^{00}}{a} e^{-(b+L(\vec{a}+\vec{b})) / 2 \lambda} e^{i k b\left(1-\cos \theta_{b R}\right)}\right\}\right\rangle,
\end{aligned}
$$

where $\mathrm{f}_{a b}^{00}=\mathrm{F}_{00}^{00}(k \vec{a}, \vec{b})$, adopting the notation of ref. 16 .

We first consider the single scattering average. The instantaneous position of the scattering atom can be related to the equilibrium position $\vec{a}_{0}$, the displacement of the origin (emitting atom), $\vec{u}_{0}$ and of scattering atom, $\vec{u}_{a}$ according to

$$
\vec{a}=\vec{a}_{0}+\vec{u}_{a}-\vec{u}_{0}=\vec{a}_{0}+\overrightarrow{\Delta u} \text {. }
$$

Defining 
$g^{00}(a)=\frac{\cos \theta_{\varepsilon a}}{\cos \theta_{\varepsilon R}} \frac{\mathrm{f}_{\mathrm{aR}}^{00}}{a} e^{-[(\mathrm{a}-\mathrm{L}(\overrightarrow{0}))+\mathrm{L}(\overrightarrow{\mathrm{a}})] / 2 \lambda}$

and noting that the thermal average consists of multiplying by a probability distribution for displacements and integrating over configurations 45 allows

$x_{1}=2 R e\left\langle g\left(\vec{a}_{0}+\Delta \vec{u}_{a}\right) e^{i k\left[\left|\vec{a}_{0}+\Delta \vec{u}_{a}\right|-\left(\vec{a}_{0}+\Delta \vec{u}\right) \cdot \hat{R}\right]}\right\rangle$.

For displacements $\left|\Delta \vec{u}_{a}\right| \ll\left|\vec{a}_{0}\right|$ we can expand

$\left|\vec{a}_{0}+\Delta \vec{u}_{a}\right|=a_{0}+\vec{a}_{0} \cdot \Delta \vec{u}_{a}+\cdots$

and

$g\left(\vec{a}_{0}+\Delta \vec{u}_{a}\right)=g\left(\vec{a}_{0}\right)+\Delta \vec{u}_{a} \cdot \nabla_{a} g\left(\vec{a}_{0}\right)+\cdots$

where forms of order $|\Delta u|^{2} / a_{0}^{2}$ have been dropped. Then we have

$x_{1}=2 R e\left\{g\left(\vec{a}_{0}\right) e^{1 k\left(a_{0}-\vec{a}_{0} \cdot \hat{R}\right)}\left\langle e^{-i \vec{k}_{a r} \cdot \Delta \vec{u}_{a}}\right\rangle\right.$

$$
\left.+e^{-i k\left(a_{0}-\vec{a}_{0} \cdot \hat{R}\right)} \nabla_{a} g\left(\vec{a}_{0}\right) \cdot\left\langle\Delta \vec{u}_{a} e^{-i \vec{K}_{a R} \cdot \Delta \vec{u}_{a}}\right\rangle\right\}
$$

where the momentum change vector, $\vec{k}_{a R}=k\left(\hat{R}-\hat{a}_{0}\right)$. Knowing relations like 
$\frac{\partial}{\partial \hat{K}_{x}} e^{-i \vec{k} \cdot \Delta \vec{u}} a=\left(\Delta \vec{u}_{a}\right)_{x} e^{-i \vec{k} \cdot \Delta \vec{u}_{a}}$

we write

$x_{1}=2 R e e^{i k\left(a_{0}-\vec{a}_{0} \cdot \hat{R}\right)}\left[g\left(\vec{a}_{0}\right)+i \nabla_{a} g\left(\vec{a}_{0}\right) \cdot \nabla_{K}\right]\left\langle e^{-i \vec{k} \cdot \nabla \vec{u}} a_{>}\right\}$

The thermal average of the phase term may be derived by following either the $x$-ray diffraction 45 theory with proper modification or the EXAFS $^{46}$ treatment:

$\left\langle e^{-i \vec{K} \cdot \Delta \vec{u}_{a}}\right\rangle=e^{-\left\langle\left(\vec{K} \cdot \Delta \vec{u}_{a}\right)^{2}\right\rangle / 2}=e^{-|\vec{k}|^{2}\left\langle\left(\hat{K} \cdot \Delta \vec{u}_{a}\right)^{2}\right\rangle / 2}$

If we identify

$\underset{\vec{a}}{\sigma_{\rightarrow}^{2}}=\left\langle\left(\hat{K} \cdot \Delta \vec{u}_{u}\right)^{2}\right\rangle$

and

$|\vec{k}|^{2}=k^{2}\left(2-2 \cos \theta_{a R}\right)$

we retrieve the Debye-Waller form (eqn (4)) but $\underset{\vec{a}}{\sigma_{\vec{a}}^{2}}$ becomes the mean square projection of relative displacement (MSPRD) upon the momentum change vector.

To proceed we need to consult some physical model for lattice vibrations which can predict $\underset{\vec{a}}{\sigma_{\vec{a}}^{2}}$. Accurate values may be derived by 
constructing the normal modes and eigenfrequencies of the solid if the geometry and bonding force constants are known. 45 while potentially useful for theoretical siudy, this approach requires far too much Information for our poorly understood surface system. The Debye model has been adapted to calculate the mean square relative displacements ${ }^{46,47}$ in good agreement with experimental EXAFS determinations. 48,33 Here we need only extend the treatment to include the predicted ${ }^{49,50}$ and observed ${ }^{51}$ surface layer dependence and anisotropy of the mean-square displacements that we can expect to encounter and the mass dependence required for an adsorbate. Our result will be a combination of the work of Allen et al 49 on mean-square displacements on surfaces, of Housley and Hess 52 on mean-square displacements in general, and of sevillano et al 47 on mean-square relative displacements, but we will trace the main points of their derivations as a means for introducing the notation.

We begin by writing the MSPRD in terms of the normal modes of a crystal with periodic boundary conditions in two directions and free surfaces in the third. Allen, Alldredge and de wette ${ }^{53}$ give the atomic displacements as

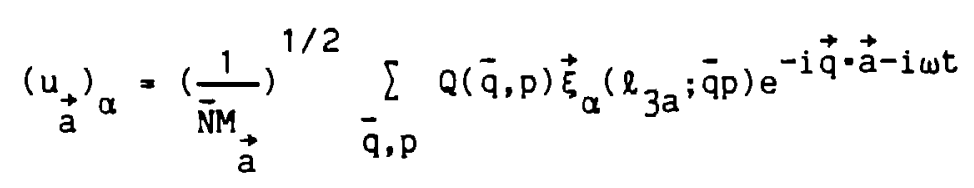

where $\bar{N}$ is the number of two dimensional lattice points, $\underset{a}{M}$ is the mass of the atom at $\vec{a}, \vec{q}$ is a two dimensional wave vector, $p$ distinguishes modes with identical $\vec{q}, Q$ is the amplitude of the mode, $\vec{\xi}_{\alpha}$ is the displacement of the atoms in the layer $\ell_{3 a}$, containing $\vec{a}$; in the mode 
$(\bar{q}, p)$, In the direction $a$, and $\omega(\bar{q}, p)$ is the mode frequency. To use this expression for the atomic displacements we expand the MSPKD, $\sigma^{2}(\vec{o}, \vec{a})$ into the sum of the mean-square displacements (MSD) of atoms $\vec{a}$ and of $\overrightarrow{0}$ minus twice their displacement correlation function (DCF):

$$
\begin{aligned}
& \left\langle\left[\left(\vec{u}_{a}-\vec{u}_{\overrightarrow{0}}\right) \cdot \hat{K}^{2}\right]^{2}=\left\langle\left(\vec{u}_{a} \cdot \hat{K}\right)^{2}\right\rangle+\left\langle\left(\vec{u}_{0} \cdot \hat{K}\right)^{2}\right\rangle\right. \\
& -2\left[\left\langle\left(\vec{u}_{a} \cdot \hat{K}\right)\left(\vec{u}_{0} \cdot \hat{K}\right)\right\rangle\right]
\end{aligned}
$$

The layer dependence of the displacements gives a layer dependent MSD:

$\left\langle\left(\vec{u}_{a}-\hat{K}\right)^{2}\right\rangle=\frac{k}{2 \bar{N} M_{a}} \sum_{\bar{q} p} \frac{\left|\vec{\xi}\left(l_{3 a} ; \vec{q} p\right) \cdot \hat{K}\right|^{2}}{\omega_{\vec{q} p}} \operatorname{coth} \frac{k \omega_{q p}}{2 k_{B} T}$

where

$\left\langle Q(\bar{q}, p) Q\left(\bar{q} p^{\prime}\right)\right\rangle=\delta_{\bar{q} \bar{q}^{\prime}} \delta_{p p} \cdot \frac{W}{2 \omega_{\overline{q p}}^{-}} \operatorname{coth} \frac{h_{\omega}}{2 k_{B} T}$

is used ${ }^{45}$ to eliminate one sum over modes and to partition the thermal energy $k_{B} T$ among the vibrational modes of energy $k_{w_{-}}$.

Next we approximate

$\left\langle\left(\vec{u}_{a} \cdot \hat{K}\right)^{2}\right\rangle=\sum_{\alpha=x, y, z} \hat{K}_{\alpha}^{2}\left\langle\left(u_{+}\right)^{2} \alpha_{\alpha}\right\rangle$.

This relation is exact in the low frequency limit for a medium with isotropic elastic constants ${ }^{45}$ or when $\hat{K}$ points along any axis $\alpha$. We can 
say that we are calculating $\left\langle u^{2}{ }_{\alpha}\right\rangle$ and approximating the projected meansquare displacement by the indicated weighted average.

The sum over modes in $\left\langle u^{2}{ }_{a}\right\rangle$ may be replaced by an integral over a frequency distribution by using 53

$\mathrm{f}_{\alpha}\left(\omega, \ell_{3 a}\right) d \omega=\frac{1}{\bar{N}} \sum_{\bar{q} P} \mid \bar{\xi}_{\alpha}\left(\ell_{3 a},\left.\bar{q} P\right|^{2} \delta(\omega-\omega-\overline{q p})\right.$.

This frequency distribution gives the density of modes of frequency $w$ with a direction displacements on atoms in layer $\ell_{3 a}$. Then

$\left\langle\left(u_{\vec{a}}\right)_{\alpha}^{2}\right\rangle=\frac{n}{2 M_{a}} \int_{0}^{\omega \max } \frac{\operatorname{coth} n \omega / 2 k_{B} T}{\omega} \mathrm{f}_{\alpha}\left(\omega_{i} \ell_{3 a}\right) d \omega$

where $\omega_{\max }$ is the highest frequency of the systems. For $\left[\mathrm{h \omega}_{\max } / 2 \mathrm{k}_{\mathrm{B}} \mathrm{T}\right]<$ 3 (i.e. moderate temperatures) we may apply a modified ${ }^{54}$ Thirring expansion ${ }^{45,52}$ of $\operatorname{coth} x=1 / x+x / 4$ to write

$\left\langle\left(u_{a}\right)_{\alpha}^{2}\right\rangle=\frac{k_{B} T}{M_{\vec{a}}}\left\langle w_{\alpha}^{-2}\left(l_{3 a}\right)\right\rangle+\frac{n^{2}}{16 M_{\vec{a}} k_{B} T}\left\langle\omega_{\alpha}^{0}\left(l_{3 a}\right)\right\rangle$

where the moments of the frequency distribution,

$\left\langle\omega_{\alpha}^{n}(l)\right\rangle=\int_{0}^{\omega} \max f_{\alpha}(\omega, \ell) \omega^{n} d \omega$

have been introduced. 55 since $\left\langle\omega_{\alpha}^{0}\left(\ell_{3 a}\right)\right\rangle=1$ the mean-square displacement in this form requires only one material parameter, the Inverse second moment of the frequency distribution. 
If we repeat these steps for the displacement correlation function, we will find 46

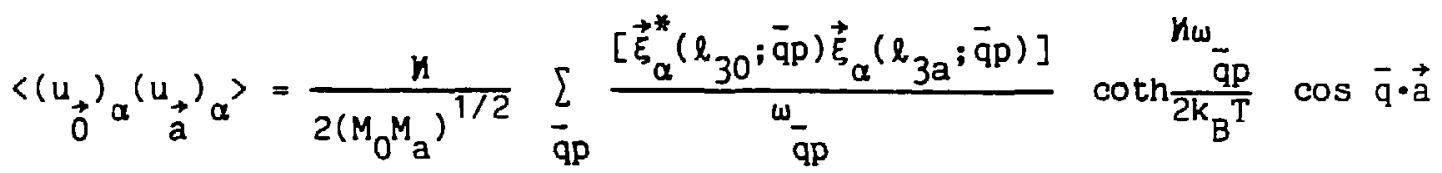

If we suppose that the moments of the frequency distribution are insensitive to any differences in the density of the modes with the direction of $\vec{q}$, we may replace $\cos \vec{q} \cdot \vec{a}$ by its spherical average 47 sinqa/qa.

To proceed we need a model for the frequency distribution and a connection between $|\bar{q}|$ and $\omega_{\bar{q} p}$. Based on the success reported for EXAFS we adopt the Debye model and set

$f_{\alpha}\left(w, l_{3 a}\right)=\frac{1}{3}\left[\frac{9 w^{2}}{\left[\omega_{D}\left(l_{3 a}\right)\right]_{\alpha}^{3}}\right]$

and $q=w\left(q_{D} / \omega_{D}\right)$ where $k_{\omega_{D}}=k_{B} \theta_{D}, q_{D}=\left(6 \pi^{2} \rho\right)^{1 / 3}$. The Debye temperature $\theta_{D}$ is a material parameter and $\rho$ is the number of atoms per unit volume. Directional, layer dependent Debye temperatures are no more than an expression of the direction and layer dependence of the moments of the frequency distribution:

$\left[k_{B} \theta_{D}\left(\alpha, l_{3 a}\right)\right]^{2}=3\left\langle w_{a}^{-2}\left(l_{3 a}\right)\right\rangle^{-2} k^{-2}$

Under these conditions we have 
$\left\langle\left(u_{a}\right)_{\alpha}^{2}\right\rangle=\frac{3 h^{2}}{k_{B} \theta_{D}\left(\alpha, l_{3 a}\right) M_{a}}\left[\frac{T}{\theta_{D}\left(\alpha, l_{3 a}\right)}+\frac{\theta_{D}\left(\alpha, l_{3 a}\right)}{48 T}\right]$

and, if atoms $\vec{a}$ and $\overrightarrow{0}$ are in the same layer,

$\left.\gamma_{\alpha}(0, a)=\left\langle\left(u_{0}\right)_{\alpha}\left(u_{a}\right)_{\alpha}\right\rangle=\frac{3 h^{2}}{\left(M_{a} M_{0}\right)^{1 / 2}} \frac{1}{k_{B} \theta_{D}\left(\alpha, l_{3 a}\right.}\right)$

* $\left\{\frac{T}{\theta_{D}\left(\alpha, l_{3 a}\right)} \frac{\operatorname{si}\left(a_{D} a\right)}{a_{D}^{a}}+\frac{\theta_{D}\left(\alpha, l_{3 a}\right)}{16 T}\left[\frac{\sin q_{D} a-a_{D} a \cos a_{D}{ }^{a}}{\left(a_{D} a\right)^{3}}\right]\right\}$

where

$\operatorname{Si}\left(q_{D} a\right)=\int_{0}^{q_{D} a} \frac{\sin x}{x} d x=\frac{\pi}{2}-\frac{\cos q_{D} a^{a}}{q_{D} a^{a}}-\frac{\sin q_{D}{ }^{a}}{\left(q_{D} a\right)^{2}}$.

The sine integral may be calculated with a numerical

form, but the asymptotic form is accurate to $1 \%$ for even the smallest $q_{D} a$ of physical interest, except $a=0$ where of course the DCF must cancel the MSD. For atoms in separate layers we know no better than to average the Debye temperatures.

We can also get low temperature limit formulas which overlaps the high temperature forms near $T=0.2 \theta_{D}$ :

$\left\langle\left(u_{a}\right)_{a}^{2}\right\rangle=\frac{3 n^{2}}{M_{a} \rightarrow k_{B} \theta_{D}\left(\alpha, l_{3 a}\right)}\left[\frac{1}{4}+\frac{T^{2}}{\left[\theta_{D}\left(\alpha, \ell_{3 a}\right)\right]^{2}} \frac{\pi^{2}}{6}\right]$ 
and

$$
\gamma_{\alpha}(\overrightarrow{0}, \vec{a})=\frac{3 h^{2}}{\left(M_{0}^{M} M_{a}\right)^{1 / 2} k_{B} \theta_{D}\left(\alpha, l_{3 a}\right)}\left[\frac{\pi T \operatorname{coth}\left[\frac{\pi T q_{D}{ }_{\theta_{D}\left(\alpha, l_{3 a}\right.}}{2 q_{D} a \theta_{D}\left(\alpha, l_{3 a}\right)}\right.}{2\left(q_{D} a\right)^{2}}\right]
$$

The first result is standard, and the second may be proven by expanding cotangent a power series and integrating terms. Taken together these two limits are adequate to represent the correlated Debye model as illustrated by corparison to numerical integrations in Fig. 2 .

To summarize our results then we write

$\underset{\vec{a}}{\sigma^{2}}=\sum_{\alpha=x, y, z} \hat{k}_{\alpha}^{2}\left[\left\langle\left(u_{\vec{a}}\right)_{\alpha}^{2}\right\rangle+\left\langle\left(u_{\overrightarrow{0}}\right)^{2}\right\rangle-2 \gamma_{\alpha}(\vec{a}, \overrightarrow{0})\right]$

where $\left\langle\left(u_{\vec{a}}\right)_{\alpha}^{2}\right\rangle$ is given by eqn. (6) or (8) and $\gamma_{\alpha}(\vec{a}, \overrightarrow{0})$ by eqn. (7) or (9). Each layer is characterized by three directional Debye temperatures, $\theta_{n, \alpha^{\circ}}$. The studies by Allen and DeWett $^{49}$ and by Clark et al ${ }^{50}$ may be used to reduce the number of free parameters. From the first work we may connect the Debye temperatures in the three crystal directions to reproduce the ratios of mean-square displacements for the theoretical crystals of Allen and Dewett. From the second work, we may cause the difference between surface layer and bulk Debye temperatures, which we may assume to be known, to decay such that the mean-square displacements approach the bulk values exponentially in three or four layers.

We have accounted for the changes in the vibrational amplitudes due to the free surface boundary conditions, but both studies cited above assume all layers have the same mass. Fortunately, Allen, Alldredge, 
and DeWett ${ }^{53}$ have considered just the problem of mass change, and they have demonstrated that, under quite general conditions, the mean-square displacement at high temperatures must be independent of mass, while at lower temperatures,

$\left\langle u_{\alpha}^{2}\right\rangle^{\prime}=\left(\frac{M}{M},\right)^{1 / 2}\left\langle u_{\alpha}^{2}\right\rangle$

where $\left\langle u_{a}^{2}\right\rangle$ is the surface MSD for a pure crystal of atomic mass $M$ and the primes indicate a hypothetical mass change of the surface layer without change in force constants. Since the mass dependence is smooth with temperature we may incorporate the adsorbate mass dependence ty noting that a Debye temperature written as

$\theta_{D}=\frac{C_{D}}{k_{B}}\left(\frac{D}{M}\right)^{1 / 2}$

where $C_{D}$ is a non-physical Debye spring constant, will reproduce the high and low temperature limit mass dependence of the mean-square displacements.

We recognize that the Debye model for the correlated motion of surface atoms is physically untenable: a true Debye model describes low frequency modes in an isotropic continuum particularly relevant to low temperatures. This does not prevent the Debye model from serving as an interpolating form for the mean-square projected relative displacements.

To complete our thermal average of single scattering we must consider the gradient terms in eqn. (5). For our particular form of the MSPRD, the gradient on momentum change has a simple form 
$\nabla_{\vec{K}} \exp \left[-|\vec{k}|^{2} \sigma_{a}^{2} / 2\right]=\exp \left[-|\vec{k}|^{2} \sigma_{\vec{a}}^{2} / 2\right] \cdot[-\vec{k}]$

where

$\vec{k}_{\alpha}=K_{\alpha}\left[\left\langle\left(u_{a}\right)_{\alpha}^{2}\right\rangle+\left\langle\left(u_{0}\right)_{\alpha}^{2}\right\rangle-2 \gamma_{\alpha}(\overrightarrow{0}, \vec{a})\right]$

The direction of this gradient is skewed away from $\vec{K}$ towards directions with the largest MSPRD. The gradients of the amplitude with respect to the separation vector $\vec{a}$ are

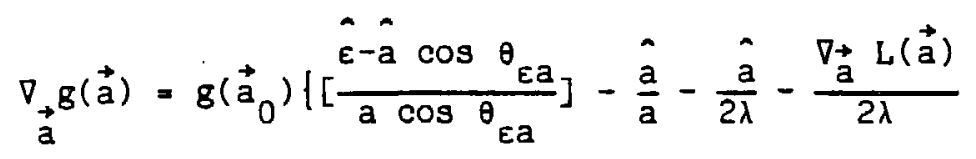

$$
+\frac{\cos \theta_{\varepsilon a}}{a \cos \theta_{\varepsilon R}} e^{-[(a-L(\overrightarrow{0}))+L(\vec{a})] / 2 \lambda}\left[k f_{a R}^{10} \hat{a}+f_{a R}^{01} \frac{\left(\hat{R}-\hat{a} \cos \theta_{a R}\right)}{a}\right]
$$

For the purpose of discussion we may dispense with the anisotropy in the MSPRD and write $\vec{k}=\sigma_{\vec{a}}^{2} \vec{K}$ with $\underset{\vec{a}}{\sigma_{\vec{a}}^{2}}$ representing the orientational average of $\underset{\vec{a}}{\sigma_{\vec{a}}^{2}}$ Then using $\vec{K}=k(\hat{R}-\hat{a})$

$x_{1}=2 R e\left(e^{i k a_{0}\left(1-\cos \theta_{a r}\right)} e^{-k^{2}\left(1-\cos \theta_{a R}\right) \sigma_{a}^{2}}\right.$

* $\left[g^{00}\left(\vec{a}_{0}\right)-\frac{i k \sigma_{a}^{2}}{a}\left\{g^{00}\left(\vec{a}_{0}\right) \frac{\sin \theta_{\varepsilon a}}{\cos \theta_{\varepsilon a}} \sin \theta_{a R} \cos \phi_{\varepsilon a R}\right.\right.$ 


$$
\begin{aligned}
& -g^{00}\left(\vec{a}_{0}\right)\left(1-\cos \theta_{a R}\right)\left(1+\frac{a}{2 \lambda}\right)-g^{00}\left(\vec{a}_{0}\right)\left[\hat{K} \cdot \nabla_{a} L(\vec{a})\right] \frac{1}{2 \lambda} \\
& \left.-k a g^{10}\left(\vec{a}_{0}\right)\left(1-\cos \theta_{a R}\right)+g^{01}\left(\vec{a}_{0}\right)\left(\sin ^{2} \theta_{a R}\right)\right\}
\end{aligned}
$$

where we use $g^{\mathrm{nm}}\left(\vec{a}_{0}\right)$ to represent $g\left(\vec{a}_{0}\right)$ with $f_{a R}^{n m}$ replacing $f_{a R}^{00}$. The derivative scattering factors $\mathrm{f}_{\mathrm{aR}}^{10}$ and $\mathrm{f}_{\mathrm{aR}}^{01}$ are described in ref. 16. In view of $A e^{i \delta k}=A(1+i \delta k)$ we may consider all of the terms containing $\mathrm{g}^{00}\left(\vec{a}_{0}\right)$ as phase corrections and, as they are linear in $k$, they represent shifts in the geometrical path-length difference. A correspondingly correction term has been predicted in EXAFS. ${ }^{56}$ The shift is very small, $\delta \leq\left(\sigma^{2} / a\right)$ unless soft materials, high temperatures, or very short bond lengths are to be investigated. The correction containing $g^{10}\left(\vec{a}_{0}\right)$ is $k_{\sigma}^{2+2}-1.0$, times the radial spherical wave correction shown in ref. 16 to be small. The last correction will be zero for forward or backscattering and will peak for $\theta_{a R}-20^{\circ}$, where the scattering amplitude changes most rapidly with scattering angle. However this correction will always be a small fraction of the curved wavefront corrections. The analogous correction for EXAFS has been studied within the plane-wave, multiple scattering theory; ${ }^{57}$ our work ere suggests that curved wave front corrections are much more significant.

For the double scattering term we will ignore the gradient terms entirely so that

$x_{2}(a, b)=x_{2}\left(\vec{a}_{0}, \vec{b}_{0}\right)\left\langle e^{1 \vec{k}_{a b} \cdot \Delta \vec{u}_{0 a}} e^{i \vec{k}_{b R} \cdot \Delta \vec{u}_{a b}}\right\rangle$ 
$=x_{2}\left(\vec{a}_{0}, \vec{b}_{0}\right) e^{-\left\langle\left[\left(\vec{k}_{a b} \cdot \Delta \vec{u}_{0 a}\right)+\left(\vec{k}_{b R} \cdot \Delta \vec{u}_{a b}\right)\right]^{2}>/ 2\right.}$

Expanding the thermal average

$$
\begin{aligned}
& \left\langle\left[\left(\vec{K}_{a b} \cdot \Delta \vec{u}_{0 a}\right)+\left(\vec{K}_{b R} \cdot \Delta \vec{u}_{a b}\right)\right]^{2}\right\rangle= \\
& \quad\left\langle\left(\vec{K}_{a b} \cdot \Delta \vec{u}_{O a}\right)^{2}\right\rangle+\left\langle\left(\vec{K}_{b \vec{f}} \cdot \Delta \vec{u}_{a b}\right)^{2}\right\rangle+2\left\langle\left(\vec{K}_{a b} \cdot \Delta \vec{u}_{0 a}\right)\left(\vec{K}_{b R} \cdot \Delta \vec{u}_{a b}\right)\right\rangle
\end{aligned}
$$

we see that the first two terms are MSPRD as given for single scattering while the last factor represents higher order correlations:

$$
\begin{gathered}
\left\langle\left(\vec{K}_{a b} \cdot \Delta \vec{u}_{D a}\right)\left(\vec{K}_{b R} \cdot \Delta \vec{u}_{a b}\right)\right\rangle=\left\langle\left(\vec{K}_{a b} \cdot \vec{u}_{0}\right)\left(\vec{k}_{b R} \cdot \vec{u}_{a}\right)\right\rangle-\left\langle\left(\vec{K}_{a b} \cdot \vec{u}_{0}\right)\left(\vec{k}_{b R} \cdot \vec{u}_{b}\right)\right\rangle \\
-\left\langle\left(\vec{k}_{a b} \cdot \vec{u}_{a}\right)\left(\vec{K}_{b R} \cdot \vec{u}_{a}\right)\right\rangle+\left\langle\left(\vec{K}_{a b} \cdot \vec{u}_{a}\right)\left(\vec{k}_{b R} \cdot \vec{u}_{b}\right)\right\rangle
\end{gathered}
$$

As before we write these factors as

$$
2\left\langle\left(\vec{K}_{a b} \cdot \Delta \vec{u}_{0 a}\right)\left(\vec{K}_{b R} \cdot \Delta \vec{u}_{a b}\right)\right\rangle=
$$

$\left.2 \sum_{\alpha=x, y, z}\left(K_{a b}\right)_{\alpha}\left(K_{b R}\right)_{\alpha}\left[\gamma_{\alpha}(\overrightarrow{0}, \vec{a})-\gamma_{\alpha}(\overrightarrow{0}, \vec{b})-<\left(u_{a}\right)_{\alpha}^{2}\right\rangle+\gamma_{\alpha}(\vec{a}, \vec{b})\right]$ 
VIII. APPLICATION TO C(2X2)S/NI(001)

Having constructed a theory of ARPEFS we now present a preliminary assessment of its predictions. Our study cannot be complete without a thorough examination of the non-structural parameters the theory requires, but we should expect physically reasonable estimates of the parameters to reproduce most of the features of the experimental curves, allowing our current work to guide both experiment and theory toward a conclusion on the practical accuracy of ARPEFS for structure measurements. In this spirit we have applied the theory of the previous sections to recent $S(1 s)$ ARPEFS measurements on $c(2 \times 2) S / N i(001)$.

The nominal structure of $c(2 \times 2) S / N 1(001)$ is lllustrated in crosssection in figure 3. Sulfur occuples a four-fold hollow adsorption site W $1^{\text {th }}$ two neighbors in the plane of the figure and two equivalent neighbors above and below the plane of the figure. Two experimental ARPEFS measurements have been made and reported elsewhere. ${ }^{18}$ The first, whlch we will call [011], aligned both emission and polarization vectors with a bulk [011] axis, making an angle of $45^{\circ}$ with the surface normal. The second experiment, called [001] here, used normal emission with the polarization vector inclined $30^{\circ}$ from normal in a [100] direction. The proportional partial cross-section oscillations, $x(E)$, measured in these experiments is plotted in figure 4. The measured kinetic energies may be converted to wavenumbers given a value for the real part of the inner potential, $E_{0}$. Throughout this paper we will use experiment curves $x(k)$ obtained with $E_{0}=10.5 \mathrm{eV}$, close to the reported values used elsewhere ${ }^{58}$.

\section{A. Cholce of Parameters}


The non-structural parameters fall into five classes: the scattering potentials, inelastic scattering, aperture integration, thermal averaging, and numerical convergence.

Our scattering potential phase shifts are those of orders and Fadley, ${ }^{10}$ generated by them from muffin tin potentials. These potentials are real, and we do not account for the ionization of the photoemitting atom.

Our inelastic mean free path is also the value given by orders and Fadley ${ }^{10}, \lambda=(0.753 k)$, where $k$ is the electron wavevector in $A^{-1}$. This mean free path lies somewhat below the "universal curve" values. We represent the surface as a plane for the calculation of the path length in the solid, and we place this plane through the adsorbed $S$ atoms. Although some guidelines for this cholce are available in surface barrier studies ${ }^{36}$, any location above the sulfur atoms is equivalent: any attenuation of the scattered waves in the region above the surface plane is cancelled by the attenuation of the direct wave when the proportional oscillations are formed. Thus we use $L\left(\vec{a}_{j}\right)=\vec{a}_{j} \cdot \hat{z} / \hat{R} \cdot \hat{z}$. $A$ more sophisticated shape for the surface barrier is hardly justified if we persist in an isotropic mean free path.

For the aperture half-angle we will use $3^{\circ}$ and we will consider only amplitude damping, ignoring the phase shift and amplitude derivative terms for this small opening. The energy dependence of the electron analyzer used in the experimental measurements indicates that the effective opening may be smaller for high kinetic energy but we will assume a constant opening.

We use the reported ${ }^{59}$ bulk Debye temperature for $\mathrm{Ni}$ of $390^{\circ}$ appropriate for room temperature. We selected the $z$ axis Debye 
temperature for $\mathrm{N1}$ as if the $\mathrm{S}$ atoms where a layer of $\mathrm{Ni}$, adjusting the Debye temperature to give $z$ axis mean square displacement on the surface twice the bulk value. This gave a Debye temperature of $300^{\circ} \mathrm{K}$. Then the Sulfur Debye temperature was corrected for the overlayer mass dependence, giving $a \theta_{D, z}=405^{\circ} \mathrm{K}$. The $x$ and $y$ Debye temperatures for both atomic species was selected as 1.1 times the $z$ axis values, to give mean square displacements about 1.4 times the bulk values.

The size of our scattering cluster is given by comparing the maximum plane wave scattering amplitude for atoms at the edge of the cluster to an amplitude cutoff and reducing the cutoff until no significant changes can be seen in the theoretical curves. Further we have only calculated path-length differences less than $10.5 \AA$ because higher frequency oscillations in the experimental curves are not reliable; the measured curves can easily be filtered to match the theoretical range via the Fourier transform. We have included up to quadruple scattering although only in rare instances will four consecutive scattering events have a path-length difference less than 10.5A. For each scattering event, the Taylor series order, $\tau$, was selected as the lowest integer which satisfied

$$
\left(\frac{f_{\max }}{2|\vec{a}|}\right)^{\tau}<C
$$

where $f_{\max }$ is the maximum plane wave amplitude $|f(\theta, k)|$ over the complete energy range for the scattering angle $\theta$, the scattering bond length is $|\vec{a}|$ and $C$ is a constant set to 0.10 . By this means, nearest 
nelghbors and forward scattering atoms are given a higher Taylor order than more distant scatterers at more acute angles.

The calculated theoretical curves are compared to the experimental data in Fig. 5 for the [001] experiment and Fig. 6 for the [011] experiment. The geometry for both theory curves placed S 2.19A away from $\mathrm{Ni}\left(\mathrm{d}_{\perp}=1.30 \mathrm{~A}\right)$ and spaced the first and second $\mathrm{Ni}$ layers by $1.84 \mathrm{~A}$; this geometry was selected by fitting theoretical calculations like those described here to Fourier filtered data in ref. 18. The theoretical reproduction of the [001] data, Fig. 5, is good: the oscillatory structures of medium frequency are all matched with some discrepancies in smaller structures. The results for [011], Fig. 6, are not so good with significant differences occurring at $5 A^{-1}, 7 A^{-1}$ and $9 \AA^{-1}$. The features at $7 \AA^{-1}$ and $9 \AA^{-1}$ are sensitive to the number of successive forward scattering events included in the calculation, and we might expect 3ome improvement here if more accurate scattering potentials ars ised. Nevertheless, the agreement between theory and experiment is good enough to suggest that distinguishing further improvements in the theory will require a quantitative assessment of the experimental "etroducibility.

Thus encouraged, we can reexamine the theory to isolate its most significant compoitents, using the [011] geometry as our example. In Fig. 7 we compare the single-scattering curved wave results to the quadruple scattering curve. The single-scattering result has the underlying frequencies correct, of course, because the frequencies are dominated by the geometrical path-length differences and because we find, in agreement with Tong ${ }^{4}$ that the multiple scattering is primarily forward scattering, whlch focusses the single-scattered waves and shifts 
their phase without disturbing their frequencies. However the osciliation phase and amplitude cannot be correctly given in the singlescattering theory.

This point deserves further emphasis as Bullock, Fadley, and Orders $^{11}$ have questioned our previous analysis of the [011] experiment, claiming on the basis of single-scattering calculations that a great many atoms contribute to the ARPEFS curves. Their conclusions are based on comparing relative single-scattering amplitudes ignoring the focusing effect of forward scattering (as well as the correlation of vibrational motion and the aperture integration). Each neglected forward scattering event is approximately equal in amplitude to the single-scattering events that they do include. In fact if we compare the expressions for single and double scattering where the second scattering event has a scattering angle near zero, we find identical terms except for an additional factor of the forward scattering amplitude divided by the bond length. Since the forward scattering amplitude is comparable to the bond length for nearest neighbors, single and double scattering are comparable. We can see this graphically in Fig. 8 where the major backscattering event for the [011] experiment is calculated in both single and double scattering. In the [011] geometry, the $\mathrm{NI}$ atom lying directly behind the $S$ photoemitter contributes a large oscillation with a frequency near $4.4 \AA$. The curves show that the single-scattering calculation is too low by nearly a factor of two: the single-scattering calculations cannot be relied upon for relative scatter $\because 18$ amplitudes. Figure 8 also shows trat the naive analys is we presented in ref 1 is erroneous. The EXAFS-like backtransformation analysis applied in ref. 1 requires the oscillation phase to be known for the $E_{0}$ adjustment 
procedure. $^{60}$ Comparing the oscillations in Fig. 8 shows that the single backscattering phase is not close to the double scattering wave phase even though the oscillation frequency is unchanged. The [011] experiment is re-analyzed including the forward scattering effects in ref. 18.

The multiple scattering curved-wave calculations are also compared to multiple scattering plane wave results in Fig. 7 . It is evident that the curved wave corrections are essential to describe the ARPEFS oscillations. This would seem to contradict the results of Sagurton et al ${ }^{13}$, who report insignificant curved wave corrections to singlescattering calculations. However, the curved wave corrections apparent in Fig. 7 are primarily in the forward scattering direction ${ }^{17}$ and hence only appear in the multiple scattering curves, which are absent in the treatment of Sagurton et al.

We have also calculated the [011] curve with multiple scattering in the zero-order Taylor series (homogeneous wave) $\operatorname{method}^{17}$ and compare it to the higher order Taylor result in Fig. 9. The zero-order curve is quite close to the higher order one, but this is partly a consequence of the [011] geometry: no important scattering atoms are near the nodal plane in the photoemission angular distribution or near a Generalized Ramsauer Townsend resonance, the types of scattering events that we have shown require higher order treatment. 16

Also in Fig. 9 we have simulated the ARPEFS curve for a $10^{\circ}$ aperture but including only the first order aperture damping. Although some of the details of the curve will be subject to correction with more accurate aperture averiging, the size of the oscillations is still large giving considerable weight to the idea that experimental measurement 
with $10^{\circ}$ apertures could be used to determine surface structures. Assuming that a suitable electron analyzer can be constructed with this large aperture, the reduction in measurement time by an order of magnitude would be of value to the experimentalist.

Finally, in Fig. 10 we illustrate some of the effects that vibrational averaging models have on the ARPEFS. In the upper panel we have returned to the uncorrelated Debye model of Bullock et al ${ }^{11}$, but adjusting the surface Debye temperature so that the Debye-Waller $0^{2}=$ $0.01 \mathrm{~A}^{2}$ for the nearest neighbor $\mathrm{N} 1$ atoms. There are some changes in the details of the curve, but the differences are not profound. In the bottom panel we illustrate an important point: the overall magnitude of the ARPEFS curve connects the physical allowed values of inelastic mean free path and thermal vibration amplitude (assuming that the elastic scattering amplitude is reasonably accurate). If the vibrational amplitude is reduced to that predicted by the surface vibrational frequency ${ }^{13}$ in a harmonic oscillator model, $.003 \mathrm{~A}^{2}$, (corresponding to a $S$ "Debye" temperature of $725^{\circ} \mathrm{K}$ ) then the oscillation amplitude will be far too large to agree with experiment and the mean free path must be reduced by $40 \%$ as in Fig. 10. Conversely low mean free path will require a stiffer surface vibration. This ambiguity can be removed by fixing the thermal parameters with a temperature dependent ARPEFS study. Finally, we address the question, do a small number of identifiable scattering atoms contribute to the ARPEFS signal? We have contended our experimental ARPEFS curves seem always to be consistent with significant scattering from nearest neighbors and backscattering atoms only. ${ }^{1,2}$ Bullock et $a l^{11}$ and Sagurton et al ${ }^{13}$ have challenged this idea on the basis of single-scattering calculations. We have shown that 
these calculations are not adequate, but we have not shown that their conclusions are invalid. We note that every improvement in the model used by Bullock ${ }^{11}$ serves to favor backscattering and nearest neighbor scattering. Thus, the correlated Debye model gives less vibrational averaging for nearest neighbor, aperture integration damps atoms which are not nearby or backscattering, and forward scattering always accompanies backscattering while other angles not always so favored. We have calculated the ARPEFS curve including only the four nearest neighbor $\mathrm{Ni}$ atoms, the four $\mathrm{Ni}$ atoms closest to backscattering in the (011) plane lying further away from $S$ than nearest neighbors and the $\mathrm{five} \mathrm{Ni}$ atoms in the succeeding (011) plane which are also backscattering. In Fig. 11 we compare the resulting curve to the experiment. It seems clear that these atoms determine the character of the ARPEFS signal. 


\section{CONCLUSION}

We have presented a theory for quantitative calculation of the intermediate energy photoelectron diffraction oscillations which we call ARPEFS. For a complete theory we must reexamine the non-structural parameters, but we belleve the present theory provides an adequate foundation for surface structure work.

We summarize our approach as follows. We have divided the problem explicitly into two parts, a time dependent, semi-classical solid state photoabsorption problem and a stationary, cluster type muffin tin potential scattering problem. This commonly used division allows us to update the treatment of the photoemission dynamics to show that dynamic core hole screening and surface barrier refraction are smaller effects than we can hope to measure at present. The cluster scattering approach gives us close contact to the interpretation of the ARPEFS oscillations in terms of particular scattering atoms while the application of the Taylor series MQNE small-atom approximation allows economical curvedwave multiple scattering calculations with a full partial wave expansion of the potential even at these higher energies. This solution to the scattering problem and its connection to lifting operator formulations of the curved wave problem facilitate analytic aperture integration and correlated vibrational averaging of the multiple scattering series, both of which we have derived here. We have also given a method for incorporating the surface dependent vibrational anisotropy into a correlated Debye model for mean-square projected relative displacements. our work must be compared to that of Tong and co-workers ${ }^{4}$ and Fadley and his co-workers. $10,11,13$ Tong, et al have the advantage of complete summation of the multiple scattering series and of closer 
contact to surface chemistry dependent potential phase shifts via the $\mathrm{X} \alpha$ multiple scattering initial state wave functions. Both of these advantages may be crucial in the low energy regime, but for most common surface systems and in the intermediate energy range, we should achieve multiple scattering convergence easily, and the potentials should be Insensitive to mild electronic changes. The cluster apprcach allows us to introduce local physical effects such as photoion core potentials and dynamic core hole screening which do not have two-dimensional periodicity and thus are more difficult to introduce into LEED-like theories.

The single-scattering cluster approach of Fadley et al. has simplicity to recommend it and up to a point it has pedigogic value. 10 However, the focusing effect of forward scattering is a fundamental feature of photoelectron diffraction, and the scattering amplitudes predicted by single scattering are not correct.

The apparent success of single-scattering theory to interpret the ARPEFS $^{1}$ was part of our original attraction to the measurement of surface structure by this method, and we must therefore examine the utility of the technique in light of the complications introduced by multiple scattering. In the intermediate energy range, multiple scattering is primarily forward scattering so that the qualitative idea that the ARPEFS oscillations represent individual scattering atoms is unchanged if we associate each the forward scattering event with the backscattering event having the same path length. Thus for the example glven in Fig. 8, the single-scattered wave travels from the photoemitting $S$ to $\mathrm{Ni}$ and backscatters into the detector giving a path length of $4.35 \mathrm{~A}$. The double scattered wave travels from $\mathrm{S}$ to $\mathrm{Ni}$ and 
back to S, scattering into the forward direction giving a path length of 4.37A. These paths are sufficiently close that we may consider the sum of both scattering events to represent the scattering signal from the $N 1$ atom for the purpose of estimating the ARPEFS signal. The presence of the forward scattering does affect our procedure for extracting the geometrical path-length difference. For the example in Fig. 8, we may not simply use the $\mathrm{N} 1$ backscattering phase shift to derive the geometry from the oscillation frequency, but instead we must perform the two atom scattering calculation to calculate the effect of the potentials on the waves. 
APPENDIX A. FINITE ANGULAR RESOLUTION

We must calculate the angular average of the total wavefunction squared:

$I_{\text {ave }}=\int_{0}^{\alpha} \sin \theta d \theta \int_{0}^{2 \pi} d \phi\left(\psi_{0}^{*}+\psi_{1}^{*}+\psi_{2}\right)^{*}\left(\psi_{0}+\psi_{1}+\psi_{2}\right)$

Our technique is to rewrite each wavefunction in a coordinate system where the $\hat{z}$ axis pointis in to the center of the analyzer aperture, $\vec{R}_{0}$. We do this by expanding the wavefunctions in a Taylor series after the manner of ref. 17; we may then integrate the expansion variables easily. The series expansions that we shall use are finite so that our results are exact in principle. More important, for small apertures, the integrated expansion converges very quickly.

We will illustrate our method by concentrating on one cross term from the full calculation,

$I_{\text {ave }}=\int_{0}^{\alpha} \sin \theta d \theta \int_{0}^{2 \pi} \mathrm{d} \phi \psi_{0}^{*} \psi_{a}$

From ref 17 , we have

$\psi_{0}(\vec{R})=\frac{e^{i k R}}{i k R} P_{10}^{00}(\vec{R}, \hat{\varepsilon})$

and

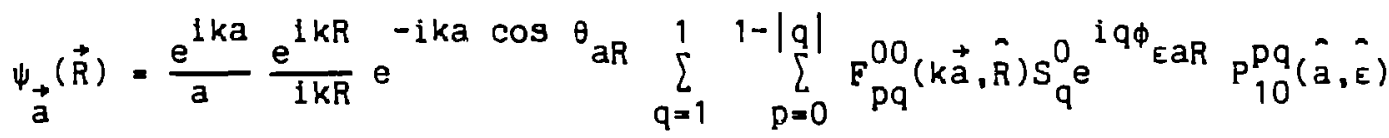


We refer to ref. 17 for the definitions of the angles and factors. Briefly, the indices $p$ and $q$ are Taylor series expansion indicies,

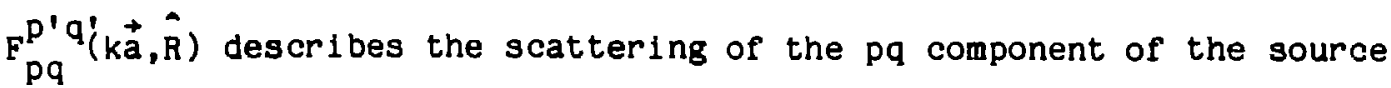
wave from a potential at $\vec{a}$ into a $p^{\prime} q^{\prime}$ component of a scattered wave in the direction $\hat{R}, S_{q}^{0}$ is a sign factor, and $P_{10}^{p q}$ is the rotation of the direct wave ints the direction $\hat{a}$.

Our first step is to write these wave functions as expansions about $\vec{R}_{0}$. The definition of $F_{p q}^{00}(k \vec{a}, \hat{R})$ contains $\hat{R}$ as an argument of a spherical harmonic quantized along the $\vec{a}$ axis, i.e. $\hat{z}$ parallel to, $\vec{a}$ $(\hat{z} / \hat{i})$. If we rotate this spherical harmonic to the $\hat{z} / / \hat{R}_{0}$ system and expand the resulting spherical harmonic in a Taylor series, we arrive at a series expansion ${ }^{17}$ in angular factors referenced to $\vec{R}_{0}$. When this series replaces the spherical harmonics in $\mathrm{F}_{\mathrm{pq}}^{00}$, we may switch the order of summation to show that

$$
\begin{aligned}
& e^{i q \phi_{E a R} S_{q}^{O} F_{p q}^{O O}(k \vec{a}, \hat{R})}=\sum_{q^{\prime}=l-\ell}^{\ell} \sum_{p^{\prime}=0}^{\ell-\left|q^{\prime}\right|} \sin \left|q^{\prime}\right|_{\theta_{R R_{0}}} e^{i q^{\prime} \phi_{a R} R}\left(\cos \theta_{R_{0}}{ }^{-1)^{p^{\prime}}}\right. \\
& F_{p q}^{p^{\prime} q^{\prime}}\left(k \vec{a}, \hat{R}_{0}\right) s_{q}^{q^{\prime}}
\end{aligned}
$$

The polar position of $\vec{R}$ in the $z / / R_{0}$ system is given by $\theta_{R R_{0}}$; the difference in the azimuth of $\vec{a}$ and of $\vec{R}$ is given by $\phi_{a R_{0}} R^{\text {. Similarly we }}$ may show

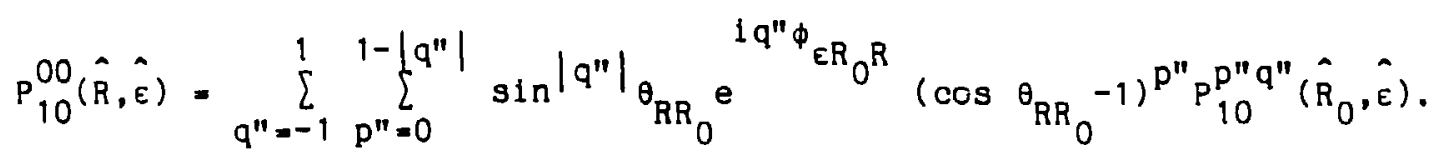


Our angular average becomes

$$
\begin{aligned}
& I_{\text {ave }}=\frac{1}{k^{2} R^{2}} \frac{e^{i k a}}{a} \sum_{q^{n}=1}^{1} \sum_{p^{\prime \prime}=0}^{1-\left|q^{n}\right|}\left[p_{10}^{p^{n} q^{\prime \prime}}\left(\hat{R}_{0} \hat{\varepsilon}\right)\right]^{*} \sum_{q=1}^{1} \sum_{p=0}^{1-|q|} P_{10}^{p q}(\hat{a}, \hat{\varepsilon}) \\
& \sum_{q^{\prime}=-\ell}^{\ell} \sum_{p^{\prime}=0}^{\ell-\left|q^{\prime}\right|} F_{p q}^{p^{\prime} q^{\prime}}\left(k a, R_{0}\right) s_{q}^{q^{\prime}} e^{-1 q^{\prime \prime} \phi_{\varepsilon R_{0}} x} e^{i q^{\prime} \phi_{a R_{0}} x} I_{p^{\prime} q^{\prime}}^{p^{\prime \prime} q^{\prime \prime}}
\end{aligned}
$$

where

$$
\begin{aligned}
I_{p^{\prime} q^{\prime}}^{p^{\prime \prime} q^{\prime \prime}} & =\int_{0}^{\alpha}[\sin \theta]\left|q^{\prime \prime}\right|+\left|q^{\prime}\right|+1[\cos \theta-1]^{p^{\prime \prime}+p} d \theta \\
& \int_{0}^{2 \pi} e^{i\left(q^{\prime}-q^{\prime \prime}\right) \phi_{x R} R} e^{-i k a \cdot \hat{r}} d \phi
\end{aligned}
$$

If we define

$$
\begin{aligned}
y=k a \sin \theta_{a R_{0}} \\
: y e^{ \pm i \phi_{x R_{0}}} \\
y_{ \pm}=y \\
z=k a \cos \theta_{a R_{0}}
\end{aligned}
$$

then we may reduce the aperture integral to

$$
I_{p^{\prime} q^{\prime}}^{p^{\prime \prime} q^{\prime \prime}}=\left(1 \frac{\partial}{\partial y-}\right)^{\left|q^{\prime \prime}\right|}\left(1 \frac{\partial}{\partial z}-1\right)^{p^{\prime \prime}+p^{\prime}\left(1 \frac{\partial}{\partial y+}\right)}\left|q^{\prime}\right| I_{00}^{00}
$$


leaving

$I_{00}^{00}=\int_{0}^{\alpha} \sin \theta d \theta \int_{0}^{2 \pi} d \phi e^{-i k \vec{a} \cdot \hat{R}}$

This integral is slosely related to an integral encountered in the study of Fraunhofer diffraction through a circular aperture. ${ }^{44}$ Expanding $\vec{a} \cdot \hat{\mathrm{R}}$ in a coordinate system where $\hat{R}_{0}$ is the $\hat{z}$ axis gives

$-i k \hat{R} \cdot \vec{a}=-i z \cos \theta-i y \sin \theta \cos \left(\phi_{x R_{0} R}-\phi_{a R_{0} R}\right)$

To separate the $\phi$ and $\theta$ variables we expand exp(-iy $\sin \theta \cos \phi)$ in a series of Bessel functions:

$e^{i k \vec{a} \cdot \hat{R}}=e^{-i z \cos \theta} \sum_{m=-\infty}^{+\infty} J_{m}\left(y \sin \theta_{R_{0} R}\right)(-i)^{m} e^{\operatorname{lm}\left(\phi_{x R} R^{-\phi_{R R} R}{ }^{\prime}\right.}$

The integration of $\phi$ selects only the $m=0$ term from this series and

$I_{00}^{00}=2 \pi \int_{0}^{\alpha} e^{-i z \cos \theta} J_{0}(y \sin \theta) \sin \theta d \theta$.

For a not too close to $\pi / 2$, we introduce the spherical Hankel functions, $h_{\ell}(z \cos \theta)$,

$I_{00}^{00}=2 \pi\left(\frac{-i z}{y}\right) \int_{0}^{a} h_{0}^{\prime \prime}(z \cos \theta)(y \sin \theta) J_{0}(y \sin \theta) d(y \sin \theta)$ 
$\frac{d}{d x}\left[x^{n+1} J_{n+1}(x)\right]=x^{n+1} J_{n}(x)$

Each partial integration raises the order of both the Bessel function and the spherical Hankel function according to

$\frac{i^{-l^{*}} h_{l}^{*}(x \cos \theta)}{(i x \cos \theta)^{l}}=\left[\frac{1}{x \cos \theta} \frac{{ }^{l}(x \cos \theta)^{l}}{{ }^{l}} h_{0}^{*}(x \cos \theta)\right.$.

Iterating the partial integrations gives

$I_{00}^{00}=2 \pi e^{-i z \cos \alpha} \frac{\sin ^{2} \alpha}{\cos \alpha} \sum_{n=0} \frac{\sin ^{2 n} \alpha}{\cos ^{n} \alpha}(-i z)^{n} d_{n}^{*}(z \cos \alpha) \frac{J_{n+1}(y \sin \alpha)}{(y \sin \alpha)^{n+1}}$

Where we have written $d_{n}^{*}(x)$ for the non-asymptotic, polynomial pait of $h_{n}^{*}(x)$. This formula is useful unless $\alpha$ approaches $\pi / 2$. For small apertures $\sin \alpha-\alpha, \cos \alpha-1$, and for $z$ not too 1 arge

$I_{00}^{00}=\alpha^{2} \pi e^{-1 z} \frac{2 J_{1}(y \alpha)}{y \alpha}$

This is the Franhoufer diffraction result discussed in section VI; we may also arrive at this result by taking the small aperture limit inside the integral and following the development of diffraction through a circular aperture. ${ }^{44}$ Since $z^{2}+y^{2}=k^{2} a^{2}$, we could expect that the interesting cases of $z$ large and a small will also have y small. This corresponds to back or forward scattering. Taking $(y \sin \alpha)$ small al jows 
$\frac{J_{n+1}(y \sin \alpha)}{(y \sin \alpha)^{b+1}}=\frac{1}{2^{n+1}(n+1) !}$

and we may sum the series to $f$ ind

$I_{00}^{00}=\pi \exp \left[-i z\left(\cos \alpha+\frac{\sin ^{2} \alpha}{4 \cos \alpha}\right)\right]\left[\frac{\sin \left(\frac{z \sin ^{2} \alpha}{4 \cos \alpha}\right.}{\left(\frac{z \sin ^{2} \alpha}{4 \cos \alpha}\right)}\right]\left(\frac{\sin ^{2} \alpha}{\cos \alpha}\right)$

Thus there is an attenuation for increasing ka $\cos \theta$ missing from the small aperture limit which follows $\sin x / x, x=\alpha^{2}$ ka $\cos \theta / 4$. This correction is 0.87 for the extreme case of $\alpha=10^{\circ}, k=12 A^{-1}$, and $a=$ 10A; for most reasonable experimental parameters we may ignore the attenuation of back and forward scattering.

Returning to the small aperture limit we can ask for the next order correction for nearest neighbor backscattering atoms. Then $z=k a \cos \theta$ will be large enough to set $d_{1}(z \cos \alpha)=1.0$ yet small enough that $\left(z \alpha^{2}\right) \ll 1$. For backscattering $y \sin \alpha-0$ and the diffraction damping terms are 1.0 so that

$I_{00}^{00}=\pi e^{-i z \cos \alpha} \frac{\sin ^{2} \alpha}{\sin \alpha}\left[1-\frac{1 z \sin ^{2} \alpha}{\cos \alpha}\right]=\pi \alpha^{2} e^{-i z\left(1+\alpha^{2}\right)}$

By $e^{-i \delta}=1$ - is, comparing to the small aperture limit we see that the amplitude correction is negligible in this case and the total second order phase correction is $\mathbf{z \alpha}^{2}$ giving a $1.5 \%$ apparent increase in bond length for a $10^{\circ}$ aperture. 
Finally we may calculate the amplitude variation corrections by differentiating

$I_{p^{\prime} q^{\prime}}^{p^{\prime \prime} q^{\prime \prime}}=\left(1 \frac{\partial}{\partial y}\right)^{\left|q^{\prime \prime}\right|+\left|q^{\prime \prime}\right|}\left(i \frac{\partial}{\partial z}-1\right)^{p^{\prime}+p^{\prime \prime}} I_{00}^{00}$

As the derivatives are straightforward, we only give one here:

$\left(i \frac{\partial}{\partial y}\right) I_{00}^{00}=2 \pi e^{-i z \cos \alpha} \frac{\sin ^{2} \alpha}{\cos \alpha}\left(y \sin ^{2} \alpha\right) \sum_{n=0} \frac{\sin ^{2 n} \alpha}{\cos ^{n} \alpha}(-i z)^{n} d_{n}^{*}(z \cos \alpha) \quad *$ $\frac{J_{n+2}(y \sin \alpha)}{(y \sin \alpha)^{n+2}}$

The presence of higher order $J_{n}(x) / x^{n}$ signals a more rapid attenuation with sine of the scattering angle; this behavior is further enhanced by the additional factor of $y \sin ^{2} \alpha$. While this predicts maximum correction for forward and backscattering, we can see in ref. 17 that the scattering factors with $q^{\prime}$ or $q^{\prime \prime}=1$ are zero in these directions; in other words the scattering amplitude does not change rapidly with angle in the regions ( $\theta-0$ and $\pi$ ) where such a change would have its maximum effect on the angle integration. 


\section{REFERENCES}

1. J.J. Barton, C.C. Bahr, Z. Hussian, S.W. Robey, J.G. Tobin, L.E. Klebanoff, and D.A. Shirley, Phys. Rev. Lett. $\underline{53}, 272$ (1983).

2. J.J. Barton, 3.W. Robey, C.C. Bahr, and D.A. Shirley, presented at the 1st Intl. Conf, on the Structure of Surfaces, Berkeley, CA., to be published in the Springer Series in Chem. Phys., 1985; J.J. Barton, C.C. Bahr, Z. Hussain, S.W. Robey, L.E. Klebanoff, and D.A. Shirley, Proc. for the Brookhaven Conf., Adv. in Soft X-ray Sci. and Technol, S.P.I.E. V. 447, 82 (1984); J.J. Barton, C.C. Bahr, Z. Hussain, S.W. Robey, L.E. KLlebanor I, and D.A. Shirley, J. Vac. Sci. Technol. A2, 847 (1984).

3. D.A. Shirley, $C R$ in Solid Stave Materials Science 10, 373 (1982).

4. S.Y. Tong, and C.H. Li, in Chemistry and Physics of Solid Surface v. III, ed. R. Vanselow and W. England, CRC Press, (1982), p. 287.

5. S.D.Kevan, Ph.D. Thesis, Univ. of Calif., Berkeley (1980).

6. P.A. Lee, Phys. Rev. B 13, 5261 (1976).

7. L.McDonnell, D.P.Woodruff, and B.W.Holland, Surf.Sci. 51,249 (1975)

8. A.Llebsch Phys.Rev.Lett. 32,1203(1974).

9. Z. Hussain, D.A.Shirley, C.H.Li, S.Y.Tong, Proc.Natl.Acad.Sci., $\underline{78}, 5293(1981)$

10. P.J. Orders and C.S. Fadley, Phys. Rev. B 27, 781 (1983).

11. E.L. Bullock, C.S. Fadley, and P.J. Orders, Phys. Rev. B 28, 4867 (1983).

12. P.A. Lee, P.H. Citrin, P. Eisenberger, and B.M. Kincaid, Rev. Mod. Phys. 53, $769(1981)$.

13. M. Sagurton, E.L. Bullock, and C.S. Fadley, Phys. Rev. B 30, 7332 (1984). 
14. S.Kono, S.M. Goldberg, N.F.T Hall, and C.S.Fadley, Phys. Rev. Lett., 41, $1831(1978)$.

15. T.Fujlkawa, J.Elec.Spectro.Rel.Phen.26,79(1982).

16. J.J.Barton and D.A.Shirley, LBL-18962, and Chapter 2.

17. J.J.Barton and D.A.Shirley, LBL-18963, and Chapter 3.

18. J.J.Barton, C.C.Bahr, S.W.Robey, Z.Hussain, and D.A.Shirley, LBL14993, and Chapter 7 ..

19. C. Noguera, D. Spanjaard, and J. Friedel, J. Phys. F 9 , 1189 (1979).

20. J.B. Pendry, Low Energy Electron Diffraction. Acadenic Press, London, ( 1974$)$.

21. H.S.W. Massey, E.H.S. Burhop, and H.B. Gilbody, Electronic and Ionic Impact Phenomena, v. 1, 2nd ed., Oxford Univ. Press, London (1969).

22. D. Pines and D. Bohm, Phys. Rev. 85, 338 (1952).

23. H.A. Bethe and R. Jackiw, Intermediate Quantum Mechanics, 2nd edition, W.A. Benjamin, Reading, MA., 1968.

24. R.D.Cowan, The Theory of Atomic Structure and Spectra, Univ.Calif.Press, Berkeley, CA. (1981)

25. J.D.Jackson, Classical Electrodynamics, 2ed,Wiley, New York, (1975).

26. C.H. Li, A.R. Lubinsky, and S.Y. Tong, Phys. Rev. B 17, 3128 (1978).

27. G.D. Mahan, Phys. Rev。 B 2, 4334 (1970).

28. L.I. Schiff, Quantum Mechanics, 3rd edition, McGraw-Hill, New York 1968.

29. C. Noguera and D. Spanjaard, J. Phys. F 11, 1133 (1981).

30. E.A. Stern, B.A. Bunker, and S.M. Heald, Phys. Rev. B 21,5521 $(1980)$. 
31. J.J. Rehr, E.A. Stern, R.L. Martin, and E.H. Davidson, Phys. Rev. B 17. $560(1978)$

32. P.M.Echeni que, R.H.Richie, N.Barberán, and J.Inkson, Phys.Rev. B 23,6486 (1981); R. Ray and G.D. Mahan, Phys. Lett. 42A, 301 (1972).

33. V.A. Biebesheimer, E.C. Marques, D.R. Sandstrom, F.W. Lytle, and R.B. Greegor, J. Chem. Phys. 81, 2599 (1984);

34. G. Einning, H. Rohner, Ch. Gerber, and E. Weibel, Appl. Phys. Lett. 40, 178 (1982); J. Tersoff and D.R. Hamann, Phys. Rev. Lett. 50, 1998 (1983).

35. T. Engel and K.H. Rieder, in "Structural Studies of Surfaces," Springer Tracts in Modern Physics 91, 59 (1982).

36. N.D. Lang and W. Kohn, Phys. Rev. B I, 3541 (1973).

37. D.Pines, Phys.Rev. 92,626, (1953)

38. J.Heinrichs, Phys.Rev.B 13,1346 (1973)

39. R.S. Williams, P.S. Wehner, J. Stönr, and D.A. Shirley, Surf. Sci. 75, $215(1978)$

40. E. Rupp, in The Interference of Electrons, ed. P. Debye, Blackie, London, 1931, P. 1.

41. K.Suzuki, M.Kitagawa, Y.H.Ontsuki, Phys.Stat.Solidi(b) $82,643(1977)$

42. B.A.Bunker, and E.A.Stern, Phys.Rev.B,27,1017(1983)

43. S.Kono, S.M.Goldberg, N.F.T.Hall, and C.S.Fadley, Phys.Rev.B $22,6085,(1980)$

44. M.Born and E.Wolf, Principles of Optics, 4th ed, Pergamon, Oxford, (1970)

45. A.A.Maradudin, E.W.Montroll, and G.H.Weiss, Theory of Lattice Dynamics in the Harmonic Approximation, Academic Press, New York 
46. G. Beni and P.M. Platzman, Phys. Rev. B 14, 1514 (1976).

47. E. Sevillano, H. Meuth, and J.J. Rehr, Phys. Rev. B 20, 4908 (1979).

48 W. Bohmer and P. Rabe, J. Phys. C. 12, 2465 (1979); R.B. Greegor and F.W. Lytle, Phys. Rev. B 20, 4902 (1979).

49 R.E. Allen and F.W.DeWette Phys. Rev. 188, 1320 (1969)

50. B.C. Clark, R. Herman, and R.F. Wallis, Phys. Rev. 139, A860 (1965).

51 A.U. MacRae and L.H. Germer, Phys. Rev. Lett. 8, 489 (1962).

52. R.M.Housley and F.Hess, Phys.Rev. 146, 517(1966)

53 G.P. Alldredge, R.E. Allen, and F.W. de Wette, Phys. Rev. B $\underline{4}, 1682$ (1971); R.E. Allen, G.P. Alldredge, and F.W. de Wette, J. Chem. Pnys. 54, 2605 (1971).

54. Y. Morino, K. Kuchitsu, A. Takahashi, and K. Maeda, J. Chem. Phys. 21. 1927 (1953)

55. R.E.Allen, G.P.Alldredge, and F.W.de Wette, Phys.Rev.B 2,2570, (1970)

56. G.A.Bunker, Nucl.Instr.Methods, 207, 437, (1983)

57. N. Alberding and E.D. Crozier, Phys. Rev. B 27,3374 (1983).

58. J.E.Demuth and T.N.Rhodin, Surf.Sci.,42,261,(1974)

59. J.A. Rayne and W.G. Kemp, Phil. Mag. 1, 918 (1956).

60. P.A. Lee and J.B. Pendry, Phys. Rev. B 11, 2795 (1975). 


\section{FIGURE CAPTIONS}

Figure 1 Aperture attenuation curves for electron analyzer half-angle openings of $3^{\circ}$ ard $10^{\circ}$. The independent variable contains geometry factors from the emission direction dependent part of the scattering path length difference. For interferencbetween direct and scattered waves $\vec{\Delta}$ is the bond vector for the scattering event which immediately precedes detection; for interference between scattered waves, $\vec{\Delta}$, is the vector difference between the bond vectors for the interfering faths. The line aa gives an indication of the $k$ range typical for nearest neighbors $(2.2 \AA)$ with $\sin \theta_{\Delta}=1.0$; the line bb corresponds to similar angles but bond lengths of 7.5 A.

Figure 2. Comparison of solutions to Debye model integrals. The mean square projected relative displacement along the $z$ axis for $S$ on $\mathrm{N} 1$ is plotted versus temperature for two differen: correlation distances, $r=50 \AA$ (upper curves) and $r=-.2 \AA$ (lowe curves). The $\mathrm{z}$ axis Debye temperature for $S$ was $41 \mathrm{U}^{\circ} \mathrm{K}$ and for $\mathrm{Ni}$ it was $300^{\circ} \mathrm{K}$; and arithmetic average Debye temperature was used for the DCF. The solid curves give results for numerical integration of the required integrals, dashed-dot curves are the results from the modified Thirring expansion of ref 54 , anc she dashed line is the los temperaure limit form.

Figure 3 Cross-sectional view of a fce crystal (001) surface showing the experimental geometry for the [011] experiment. The angle-rciolving detector is along the vector labeled $\mathrm{e}^{-}([011]$ directids); the polarization vector is $E$. The geometrical path length difference is given by the bond distance from $S$ to 
a scattering $\mathrm{Ni}$ atom plus the distance from the $\mathrm{Ni}$ atom to the plane perpendicular to the emission direction and passing through the $S$ photoemitter.

Figure 4 ARPEFS modulations derived from $S(13)$ photoemission partial cross-sections. Both curves were measured from the $c(2 \times 2) S / N i(001)$ system. (a) Emission along a [011] direction ( $45^{\circ}$ from normal); (b) Emission along the crystal normal.

Figure 5 Numerical simulation (dashed curve) of the [001] experimental data (solid curve)

Figure 6 Numerical simulation (dashed curve) of the [011] experimental data (solid curve)

Figure 7 Comparison of numerical simulation curves for S(1s) ARPEFS from $c(2 \times 2) S / N i(001)$ along [011]. (a) Multiple scattering, plane wave theory, (b) multiple scattering, curved wave theory, (c)single scattering, curved wave theory. All three curves used Identical geometries and non-structural parameters.

Figure 8 Comparison of ARPEFS single and double scattering for [011] emission, but only including the scattering from the $\mathrm{N} 1$ atom directly behind the $s$ from the detector. Thin curve, single backscattering from Ni; Thick curve, single backscattering from $\mathrm{Ni}$ plus forward focusing through $\mathrm{S}$. The actual scattering angles are $173^{\circ}$ for backscattering and $7^{\circ}$ for forward scattering. 
Figure 9 As in Pigure 7 with (a) zero-order Taylor series (homogeneous wave theory), (b) full theory, (c) full theory with aperture damping corresponding to $10^{\circ}$ half angle.

Figure $10 \mathrm{As}$ in figure 7 with (a) vibrational amplitudes calculated without correlation of vibrational motion (mean square displacements instead of mean square projected relative displacements), (b) full theory, (c) same as (b) with mean free path of $.44 \mathrm{~K}$ \& and a $S$ Debye temperature of $725^{\circ} \mathrm{K}$ for all three directions.

Figure 11 Numerical simulation (dashed curve) of the [011] ARPEFS data (solid curve), but including only a very limited number of scattering atoms, as described in the text. 


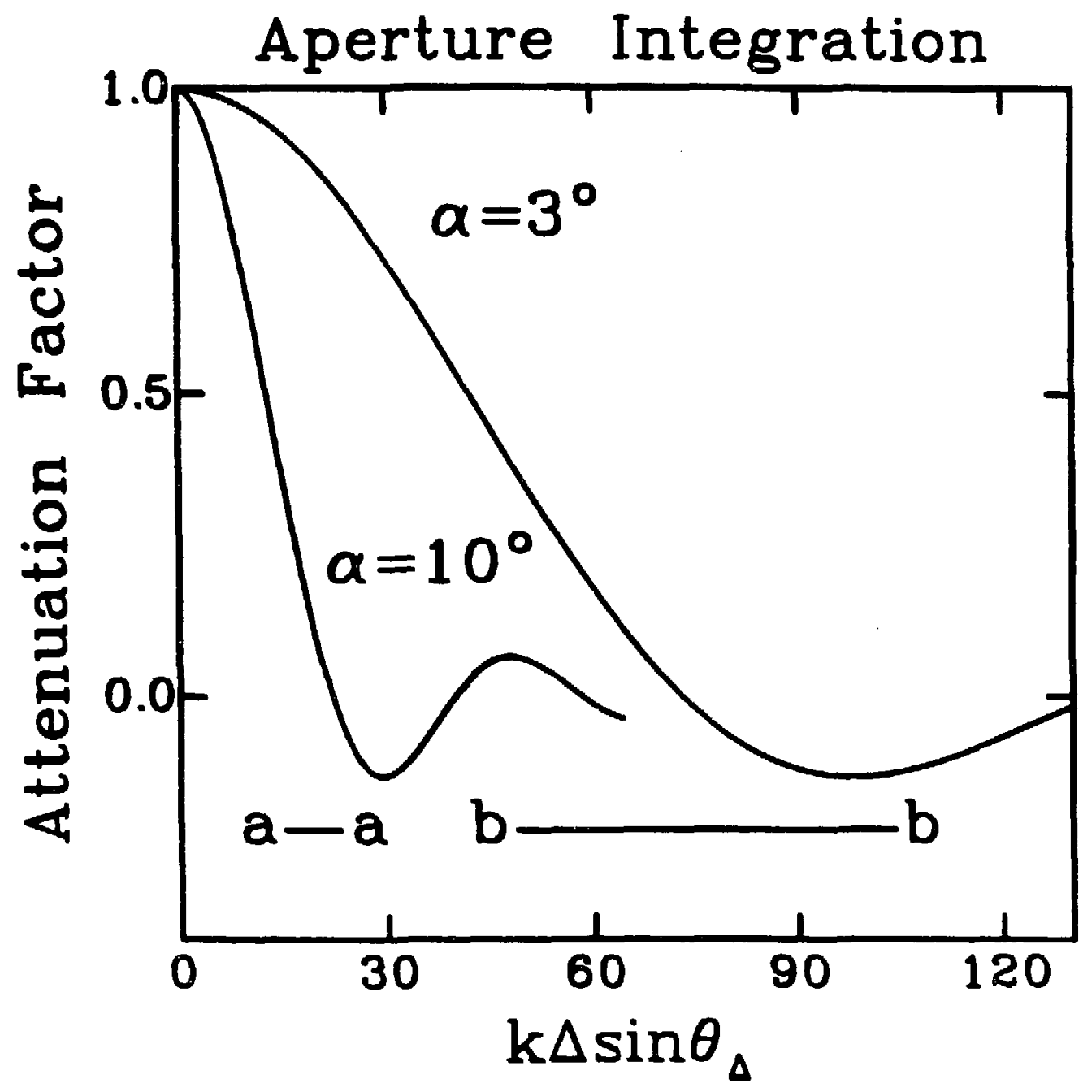

XBL 853-1832

Figure 1 


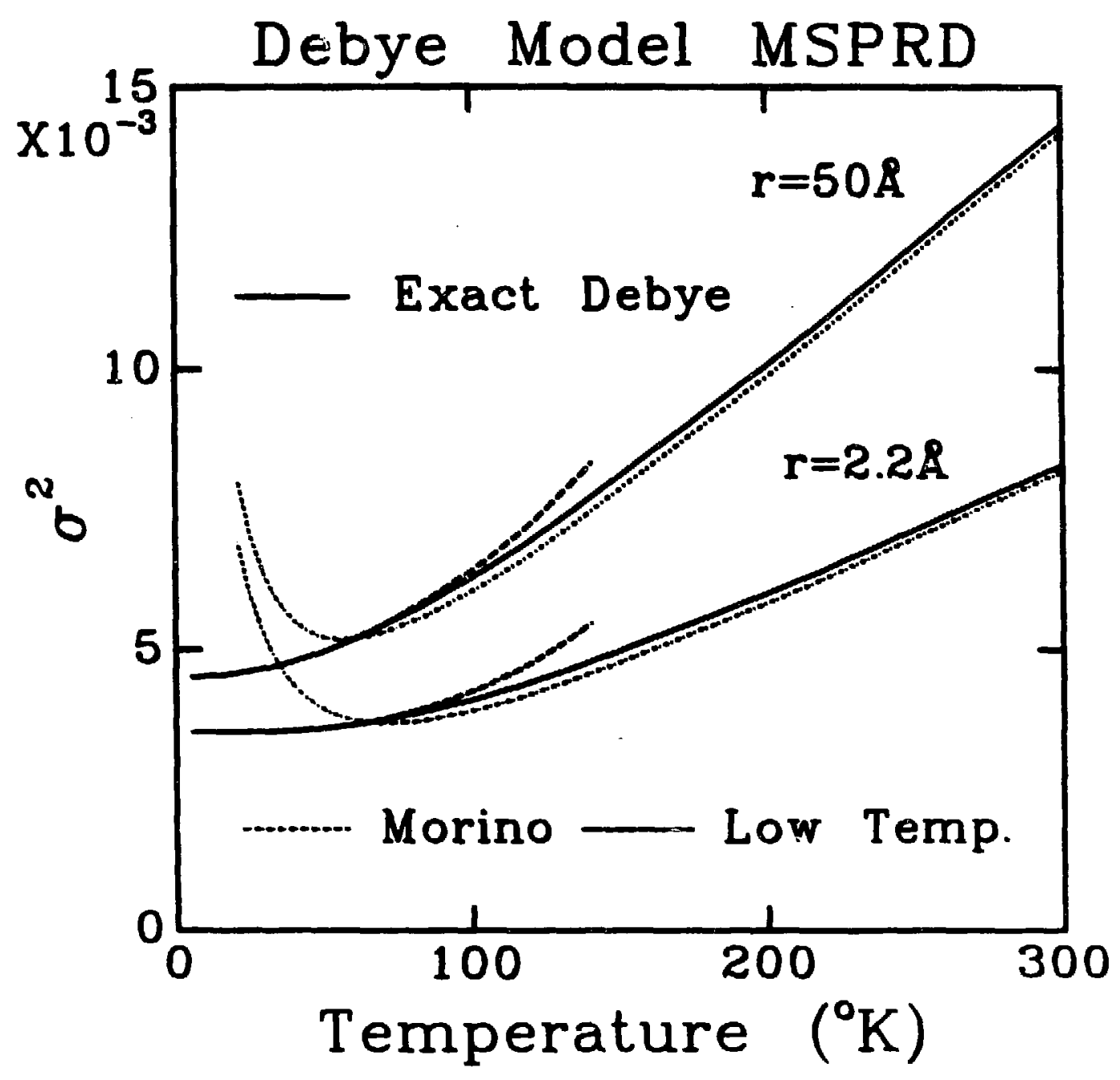

XBL 853-1839

Figure 2 

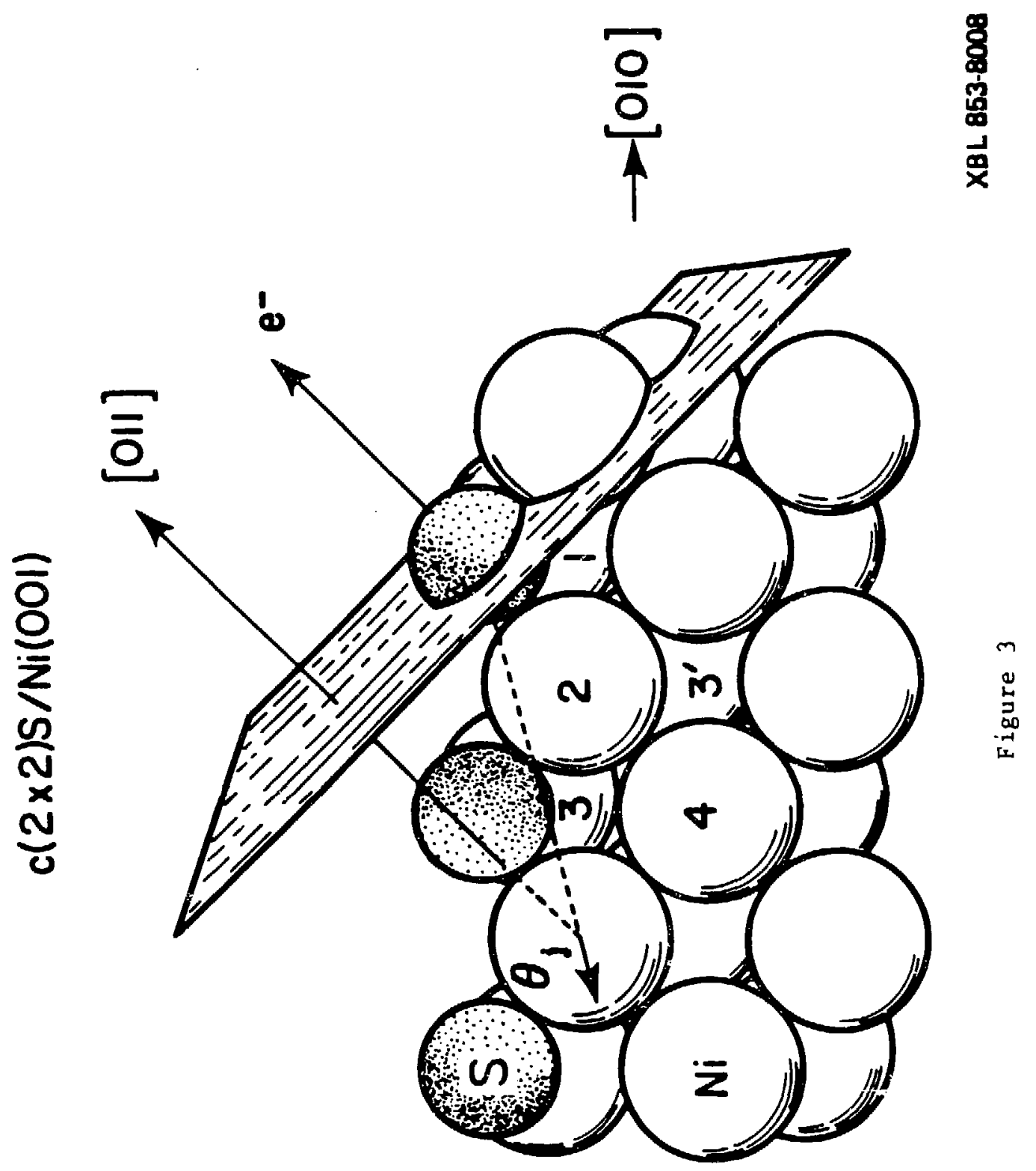


\section{Coherent Single Bunch Effects}

Single bunch longitudinal fast blowup due to microwave instability does not cause beam loss but simply lengthens the bunch until the bunch peak current falls below the threshold of the microwave instability. The coupled bunched modes calculated above have already taken into account this effect by using microwave lengthened bunches (including any lengthening due to intrabeam scattering) for calculations. [2] Transverse fast blowup instability (analogous to the longitudinal microwave instability) can cause beam loss however. Transverse and longitudinal fast. instability thresholds coincide when ${ }^{[7]}$

$$
\sigma_{\epsilon}^{0} \simeq \frac{b}{\sqrt{8 \pi} \bar{\beta}}
$$

where $b$ is the radius of the vacuum chamber and $\bar{B}$ the average beta-function in the transverse direction under consideration. If $\sigma_{\varepsilon}<\sigma_{E}^{0}$, the transverse threshold is higher than the longitudinal. Since the Deam is always slightly below longitudinal threshold in its equilibrium situation, this inequality would guarantee that the beam is safe from transverse blowup effects. For Aladdin-3 at $800 \mathrm{MeV}$ with the $515.459 \mathrm{MHz}$ PETRA cavity and 15 bunches, we have

$$
\sigma_{\varepsilon}^{0}=1.82 \times 10^{-3} \text { and }\left(\sigma_{\varepsilon}\right)_{\max } \simeq 1.67 \times 10^{-3}
$$

even when an average current of $200 \mathrm{~mA}$ is put in a single bunch. Aladdin is not expected to suffer seriously from transverse fast blowup effects.

There is the possibility of another fast transverse instibility, different from above and known as the transverse fast head-tail instability. It arises from transverse mode coupling even with zero chromaticity (synchrotron modes 0 and -1 confluence and couple with each other). The threshold for this instability is given by ${ }^{[7]}$ : 


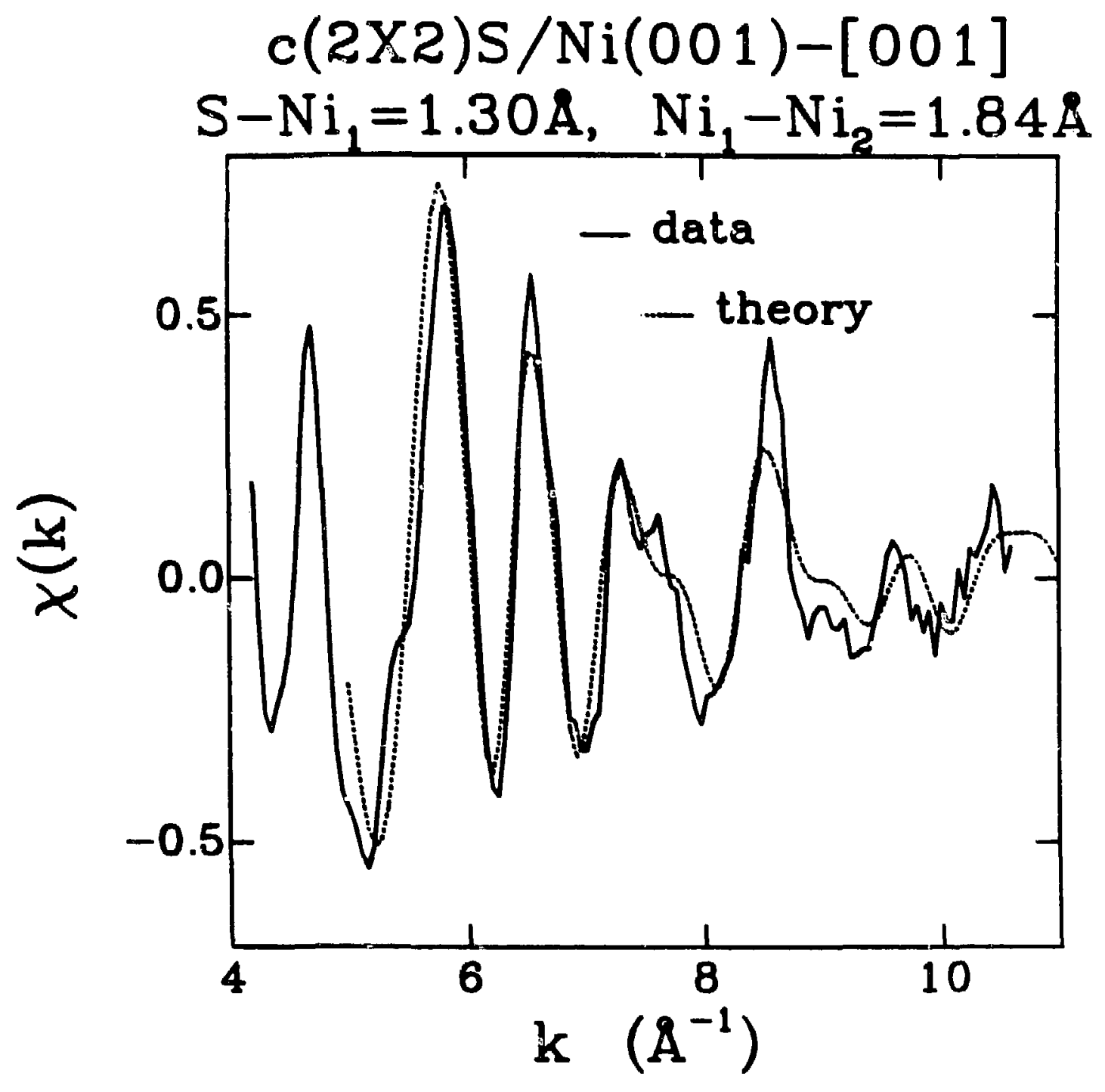

XBL 853-1820

Figure 5 


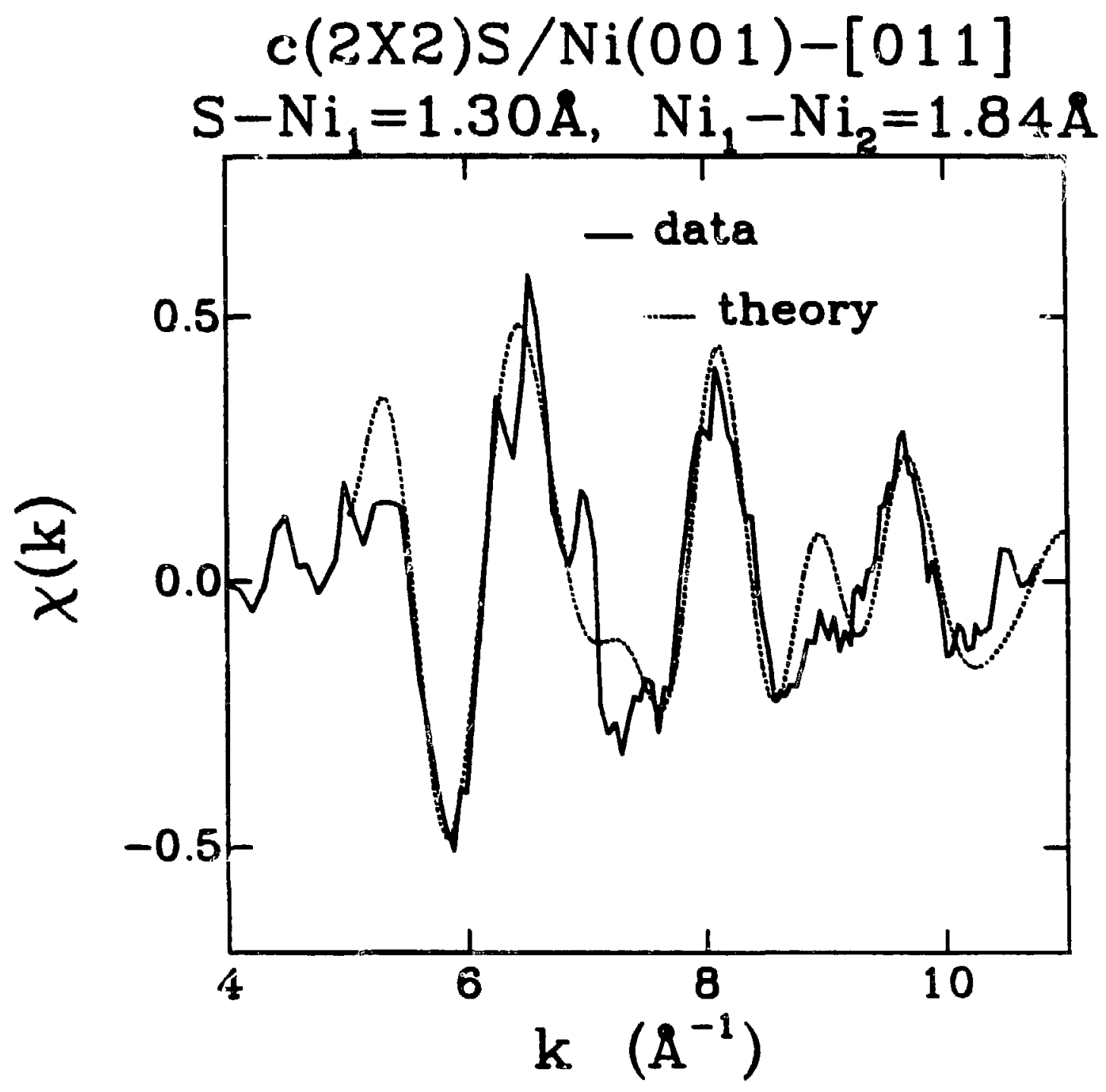

XBL $853-1818$

Figure 6 


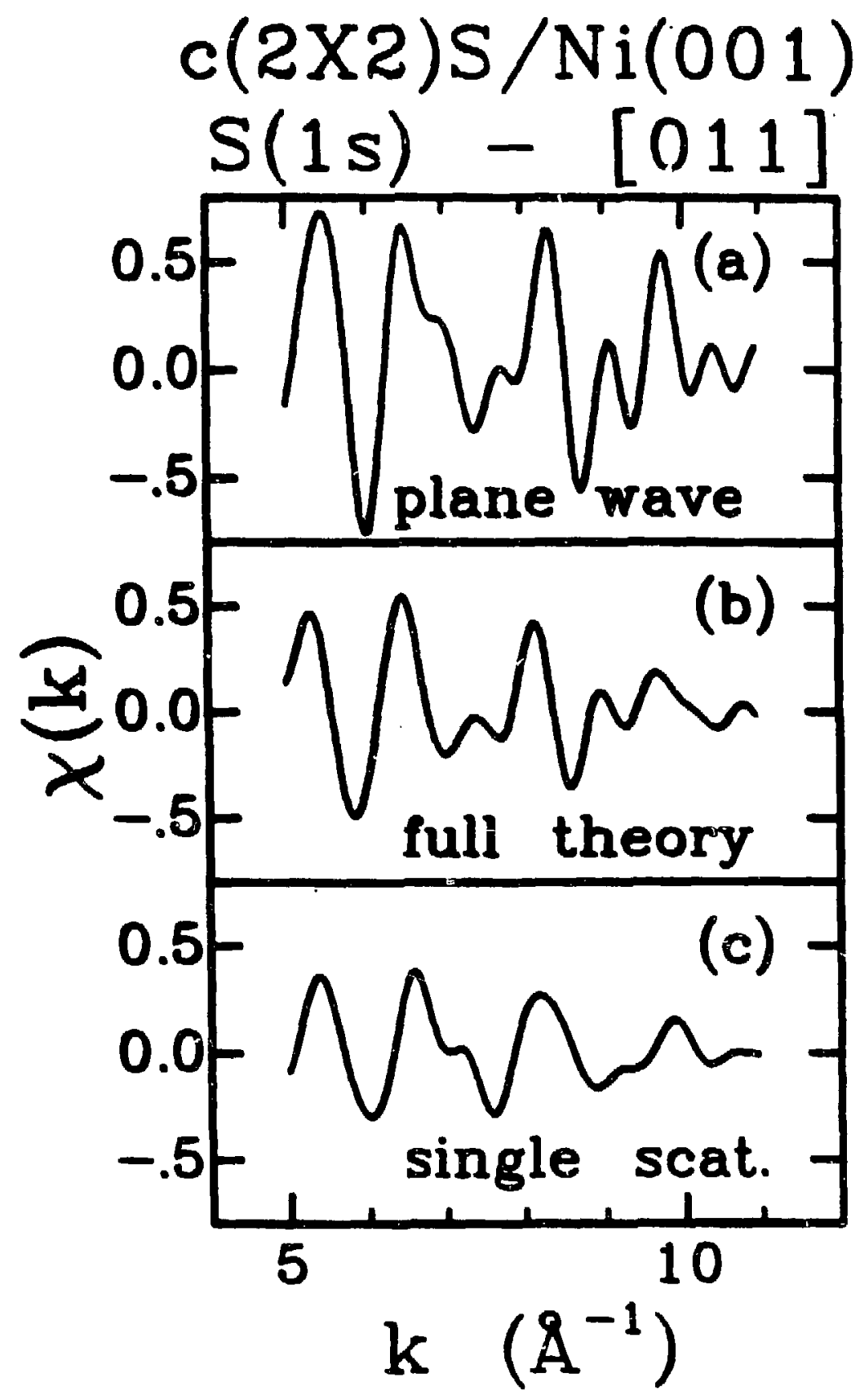

XBL 853-1828

Figure 7 


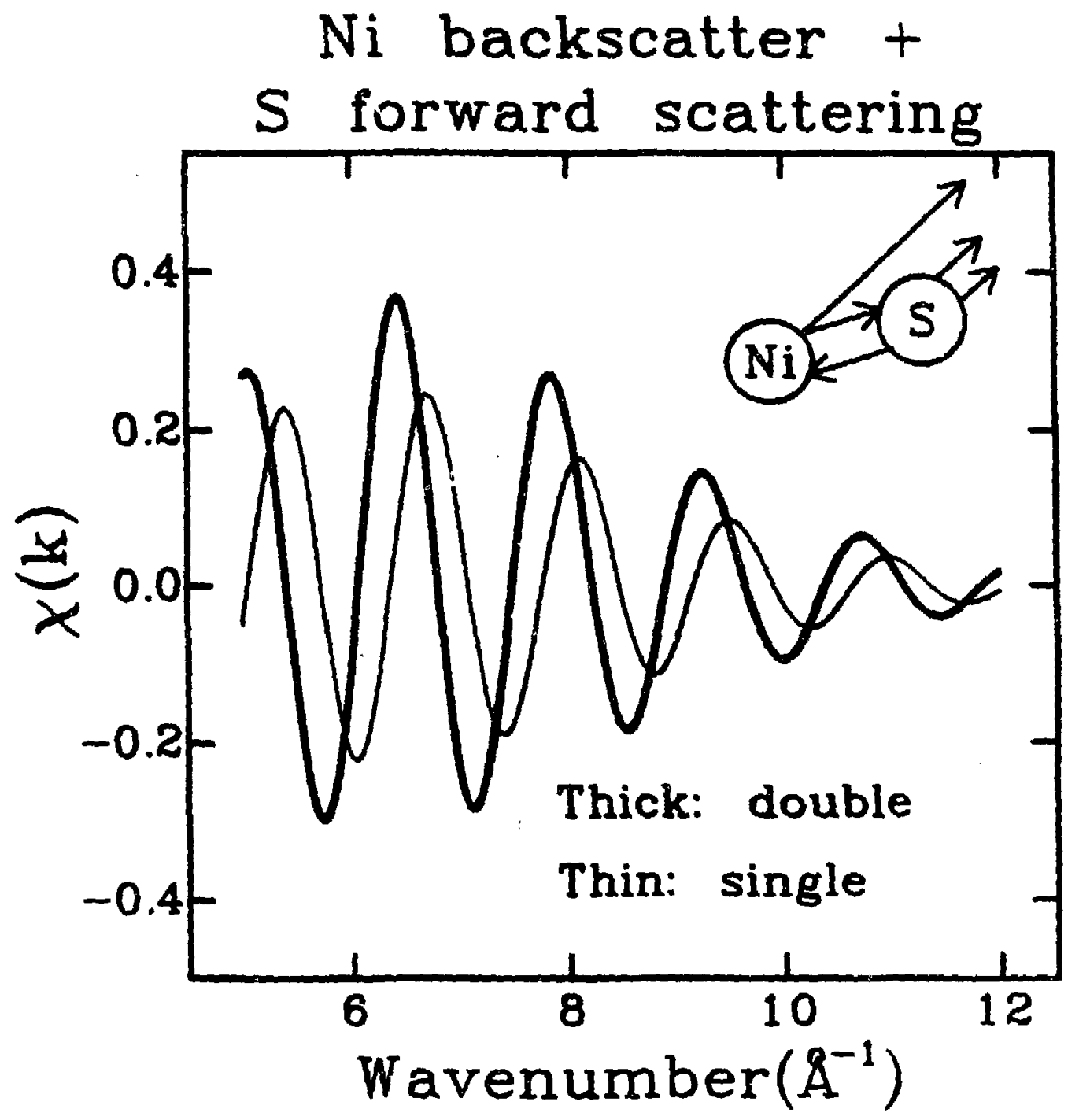

XBL 853-1837

Figure 8 


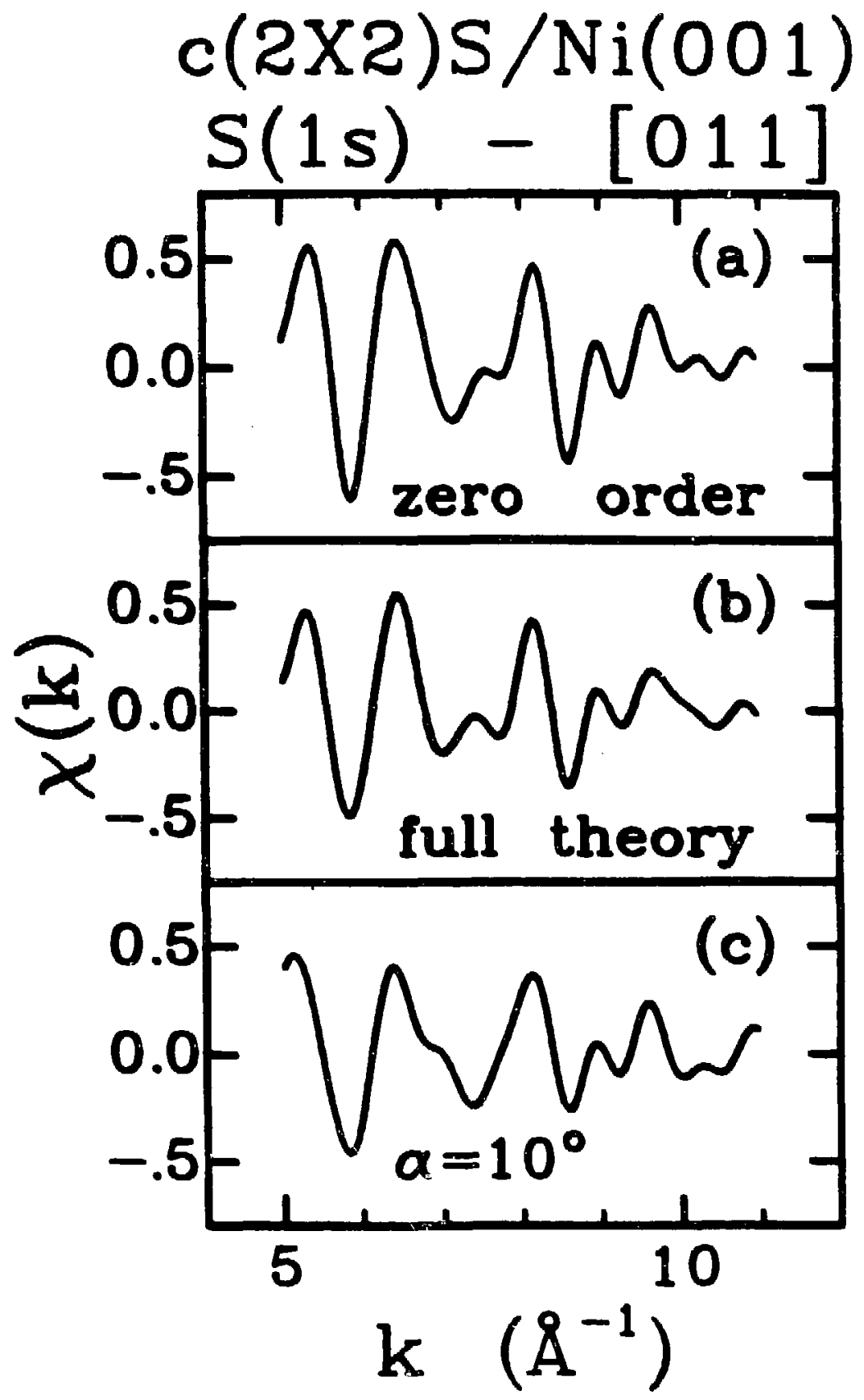

XBL 853-1831

Figure 9 


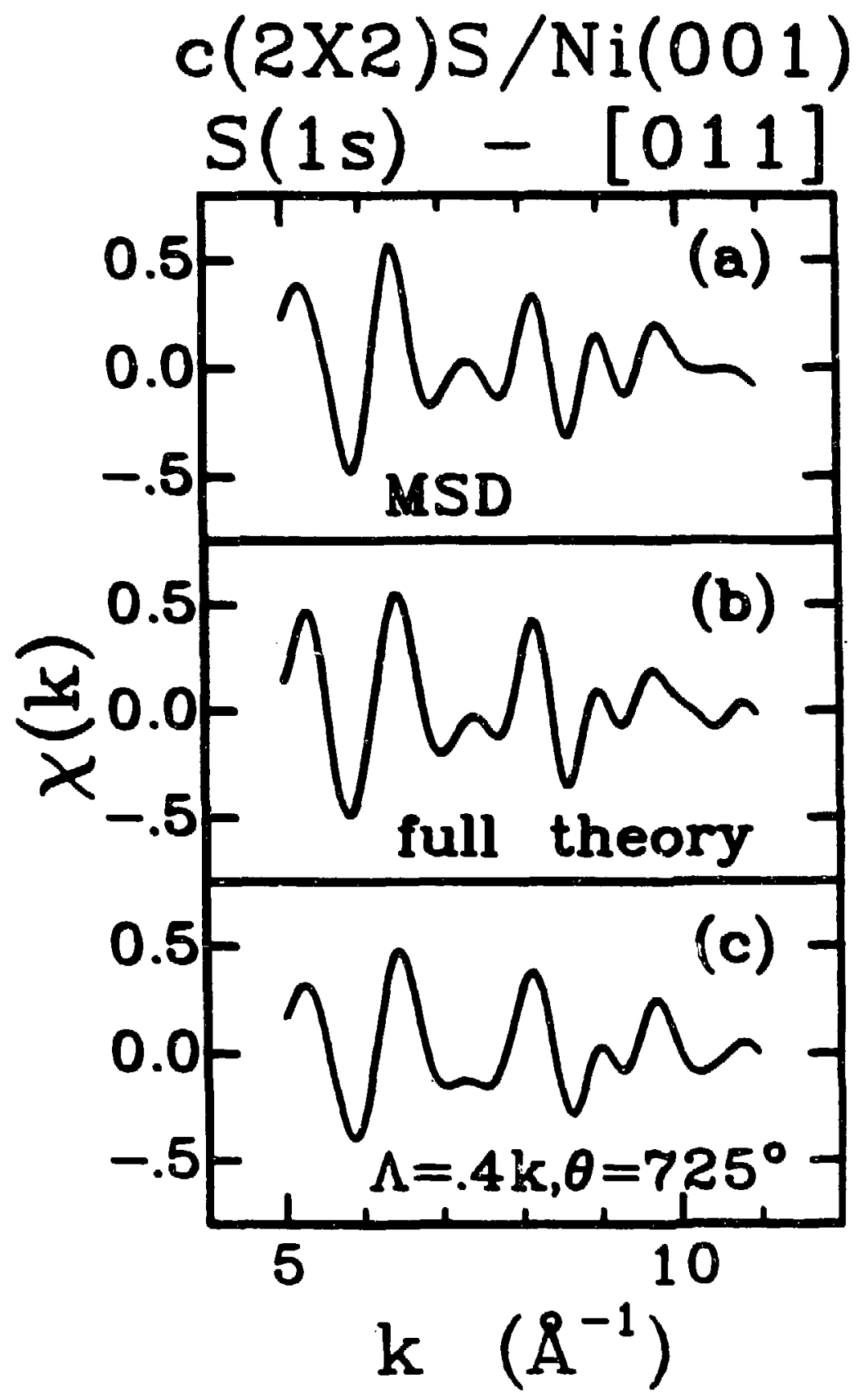

XBL 853-1829

Figure 10 


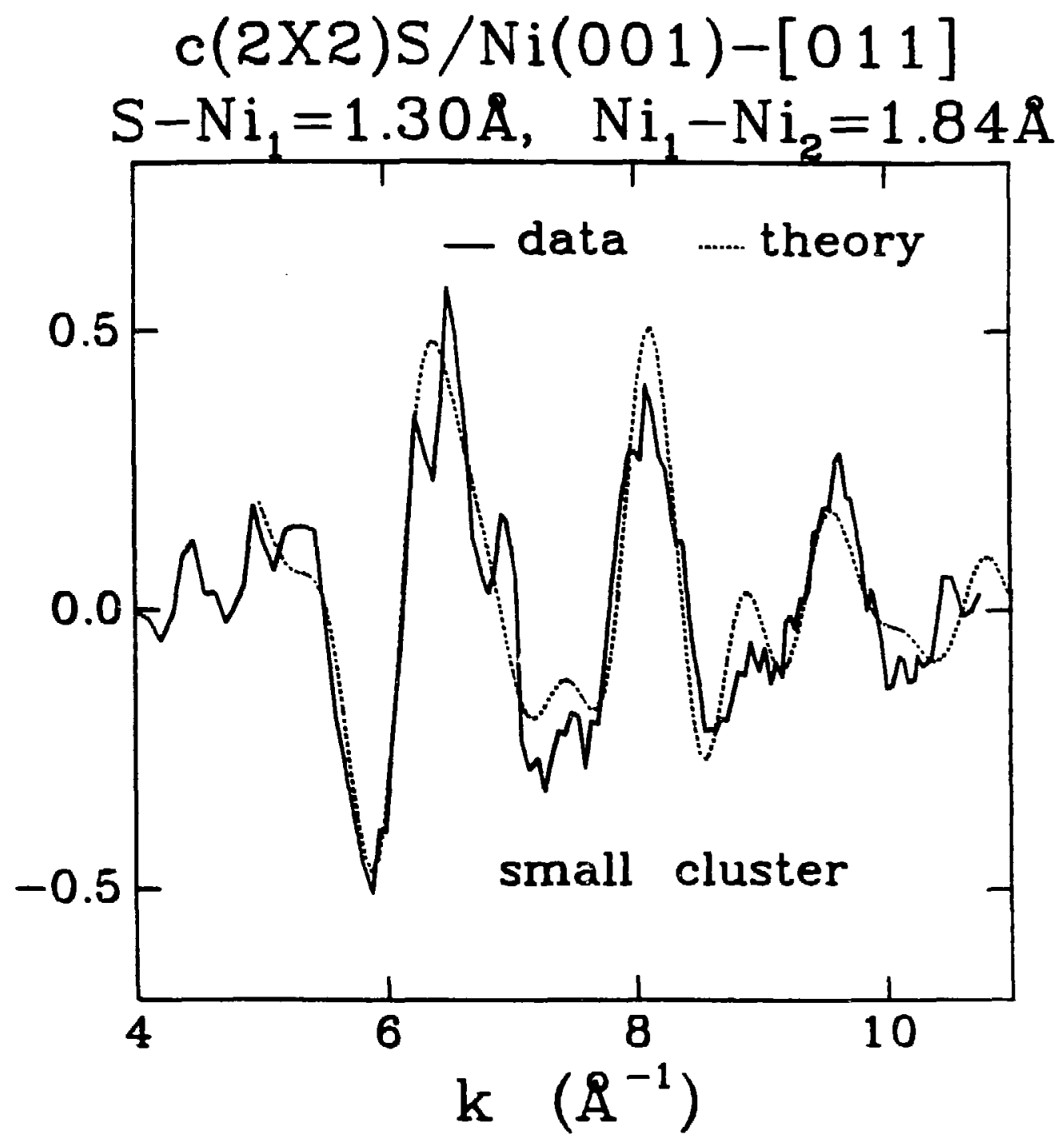

XBL 853-1838

Figure 11 
CHAPTER 6:

FOURIER ANALYSIS OF EXTENDED FINE STRUCTURE

WITH AUTOREGRESSIVE PREDICTION

\section{ABSTRACT}

Autoregressive prediction is adapted to double the resolution of Angle-Resolved Photoemission Extended Fine Structure (ARPEFS) Fourier transforms. Even with the optimal taper (weighting function), the commonly used taper-and-transform Fourier method has limited resolution: it assumes the signal is zero beyond the limits of the measurement. By seeking the Fourier spectrum of an infinite extent oscillation consistent with the measurements but otherwise having maximum entropy, the errors caused by finite data range can be reduced. Our procedure developed to implement this concept applies autoregressive prediction to extrapolate the signal to an extent controlled by a taper width. Difficulties encountered when processing actual ARPEFS data are discussed. A key feature of this epproach is the ability to convert imiroved measurements (signalto-noise or point density) into improved fourier resolution. 


\section{INTRODUCTION}

Fourier transformation is a basic tool for spectroscopic data analysis in several contexts. Typically, Fourier transformation is

used for harmonic analysis. ${ }^{1-3}$ The spectroscopic measurement records an intensity while scanning energy; the Fourier transformation converts this energy spectrum into a frequency spectrum, reporting the amplitude and phase of a series of fixed frequency sinusoids which sum to the experimental result. If the physically significant part of the measurement has a distinctive frequency dependence, the signal frequencies can be isolated from irrelevant background or noise frequencies. Synthesis of the signal frequencies then yields a new energy spectrum whose interpretation may be simpler. For example, Extended X-ray Absorption Fine Structure (EXAFS) data are usually analyzed in this manner. ${ }^{4,5}$

Conceptually, Fourier analysis yields the amplitude and phase of each individual sine wave in a series which sums to give the spectroscopic signal. Of course, sine waves continue indefinitely while spectroscopic signals typically have a limited range. If the data analysis is restricted to a Fourier transform, this mismatch inevitably leads to a broadened Fourier spectrum: wide peaks appear for dominant frequencies, but adjacent peaks may overlap and the desired separation in frequencies may not be realized. With the Fourier methous currently used in spectroscopy ${ }^{4-6}$ this finitedata-range broadening cannot be reduced by more careful measureinents within a fixed interval. Thus if the measurement range is physically 
restricted, then the ability of simple Fourier analysis to separate dominant frequencies will be limited.

Because of this broadening, the advantage of the explicit harmonic content analysis provided by a single Fourier transformation is of fset by its lowering of frequency resolution. This broadening effect is extrinsic to the data set: it is inflicted on the data by forcing a clumsy method of analysis, because we force infinite sine waves functions to reproduce a finite length data sequence. ${ }^{7,8}$ An implicit method for extracting the harmonic content (e.g., leastsquares fitting the data) would provide the required frequency resolution. It is, moreover, also possible to realize the advantage of both approaches; viz, high frequency resolution and explicit analysis, by combining regression methods and Fourier analysis. Such an approach, for a particular spectroscopic method, is the subject of this paper.

To directly analyze Angle-Resolved Photoemission Extended Fine Structure (ARPEFS), a photoelectron diffraction phenomenon useful for surface structure determination, ${ }^{9}$ we have found the frequency resolving power of the usual spectroscopic Fourier analysis to be inadequate, because the data range is limited. Fortunately, we have been able to adapt one of the new approaches to the Fourier analysis of physical measurements that allows higher fourier resolution and can trade measurement precision for Fourier resolution. We shall report and discuss an adaptation of autoregressive prediction, also known as maximum entropy spectral analysis, which improves the Fourier 
resolution by a factor of two in practical cases. Autoregressive prediction is widely used to process geophysical and acoustical measurements $1,3,10$ when estimates of power spectra are required, but only short data sequences are available. We will demonstrate that autoregressive prediction can be used to extend the effective range of sinusoidal. ARPEFS signals by an amount which increases with the signal-to-noise ratio. Although we apply this method to the analysis of ARPEFS, the method is directly applicable to EXAFS data or to other spectroscopies requiring high resolution Fourier transformations.

After ARPEFS is described in Section II, the taper-and-transform method of Fourier analysis is discussed in Section III. Autoregressive prediction is introduced in Section IV. The results are discussed in Section $y$, and a summary appears in Section VI.

\section{ARPEFS}

We shall demonstrate the autoregressive Fourier technique by applying it to ARPEFS data. In this section we briefly describe the essential physics of ARPEFS and discuss why high resolution fourier analysis is required.

Angle-resolved photoemission extended fine strusture is the oscillatory part of the photoemission current as a function of photoelectron kinetic energy. ${ }^{9}$ Photo-excitation of an adsorbate core level gives an atomic-like (direct) outgoing photoelectron wave. Direct propagation of this wave to our detector would give an overall 
atomic character to the differential cross section. Elastic scattering of this wave from substrate atoms leads to a new set of waves which can reach the detector and which interfere with the direct wave. For electron kinetic energies from about 50 to $500 \mathrm{eV}$, two conditions are met: single elastic scattering from ion cores dominates and the electron de Broglie wavelength corresponds to atomic dimensions. Thus, the interference modulation with kinetic energy can be used to derive the scattering path length and hence the position of the adsorbate atoms relative to the substrate.

The ARPEFS modulations are strongly dependent on the scattering angle, $a_{j}$, the angle betwren the photon polarization vector and scatterer, $B_{j}$, and the angle between the detector and the polarization vector, $r$. In the simplest theory, ${ }^{11}$ the modulations, $x(k)$, expressed as a function of the electron de Broglie wavenumber, $k$, are

$$
x(k)=\sum_{j} A_{j} \cos \left[k r_{j}\left(1-\cos a_{j}\right)+a_{j}\right] \cdot
$$

where

$$
A_{j}=\frac{\cos B_{j}}{\cos r} \frac{\left|f\left(a_{j}\right)\right|}{r_{j}} e^{-L_{j} / \lambda} e^{k-\sigma_{j}^{2} k^{2}\left(1-\cos a_{j}\right)}
$$

for is photoabsorption. If we call the polarization vector $\vec{\varepsilon}$, the emission vector $\vec{k}$, and the vector from the emitter atom to the $j^{\text {th }}$ scatterer $\vec{r}_{j}$, then the parameters in this formula are: 


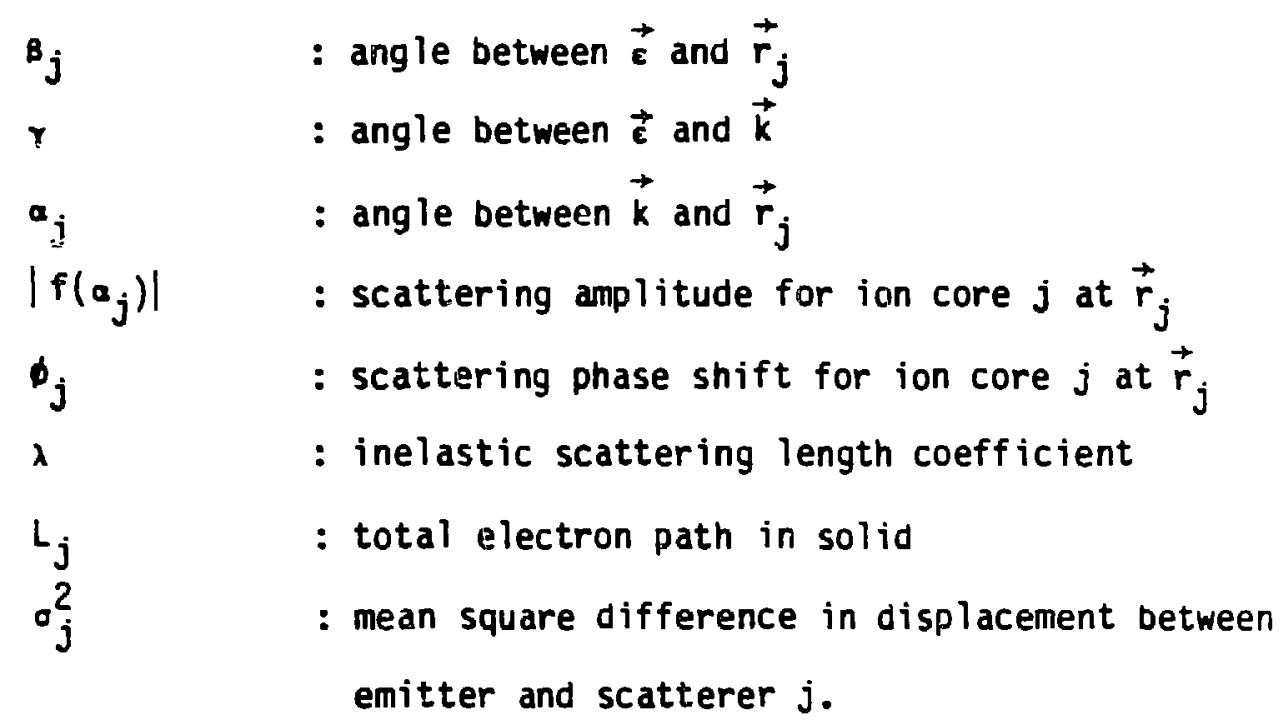

The argument of the cosine contains the geometrical information, $r_{j}-r_{j} \cos a_{j}$. If the contribution from a single scatterer can be isolated, the scattering phase shift, $b_{j}$, can be removed and the structure can be determined.

Because the single scattering theory is not valid for low wavenumber measurements and because the Debye-Waller factor, $\exp \left(-\sigma^{2} k^{2}\left(1-\cos a_{j}\right)\right)$, reduces the intensity of the oscillations for high wavenumbers, the useful ARPEFS data range typically lies between $3 A^{-1}-\mathrm{rad}$. and $12 A^{-1}$-rad. As we show in the next section this range may not be sufficient to resolve the nearest neighbor path lengths when normal Fourier analysis is applied.

III. THE TAPER-ANO-TRANSFORM METHOD

To demonstrate our Fourier method we analyzed a narmonic sum 
(Fig. la) made up of test data consisting of two sine waves with frequencies of $5 A$ and $6 A$ sampled 128 times in the interval from 4 to $11 A^{-1}$-rad. We added pseudo-random numbers to give a signal/noise ratio ${ }^{12}$ of 2.8. Two important differences between this signal and our ARPEFS data--the $k$ dependences of the amplitude $A_{j}$ and of the phase $\phi_{j}-w i l l$ be examined in Section $V$.

Direct application of the discrete Fourier transform,

$$
g(p)=\frac{1}{N} \sum_{q=0}^{N-1} G\left(\frac{q}{N}\right) e^{-i 2 \pi p q / N},
$$

to the test sequence of $N$ points [G] gives, via the Fast 5ourier Transform, ${ }^{2}$ a sparsely digitized Fourier spectrum, [g], shown in Figure 1b. The density of points in the fourier spectrum can be increased by simply appending zeros to the sequence, [G], as Figures Ic and ld illustrate, but ringing sidelobes-Gibbs oscillations-then appear, as a consequence of the finite length of the data sequence. ${ }^{1,2}$ These oscillations obscure or confuse features in the experimental fourier spectrum. They arise from the sharp truncation of the signal at the ends of the range. If $y(p)$ is the sinusoid that we would get if we could measure an infinite range of data, then our experiment gives

$$
b(p)=w(p) * y(p) \quad-\infty<p<+\infty
$$

The box function, $w(p)$, truncates the signal at the extremes of the 
measurement interval:

$$
\begin{array}{ll}
w(p)=0 & p<1 \\
w(p)=1 & 1 \leq p \leq N \\
w(p)=0 & p>N
\end{array}
$$

for $N$ measurements. The Fourier transform of $b$ is the convolution of the transform of the sine waves (delta functions) and the transform of the box $\left(\sin ^{2} x / x^{2}\right)$. The sidelobes oscillations of the box transform are then superimposed upon the delta functions.

The usual approach for reducing these oscillations is termed "taper-and-transform" spectral analysis." The sharp-edged box is replaced by a smooth weighting function whose Fourier transform does not contain large oscillations. This weighting function will broaden the Fourier spectrum as it reduces the sidelobe oscillations. Harris ${ }^{13}$ and Nuttal1 14 surveyed a variety of weighting functions and compared their performance by several criteria. For our purposes, the appropriate weighting function should have the highest possible resolution for a sidelobe-to-mainlobe ratio below the flat Fourier spectrum of the noise (assuming approximately normal distributed noise). Sidelobes falling below this level will have no more impact than the noise from the measurement. For sine waves, the Fourier signal-to-background will be the square root of half the number of data channels times the signal-to-noise ratio; this may be used as a rough guide for the weighting function selection.

As a measure of resolution we select the full width at half maximum valve and label it $\Delta r$. The width of the measurement, $\Delta k$, can 
be related to this resolution as

$$
\Delta r=\frac{2 \pi}{\Delta x} f
$$

where the factor $f$ depends on the weighting function. For a square window (no weighting) $f=1.21$, and the sidelobe is .22 times the mainlobe. Harris gives $f$ as the "6 dB resolution" and reports the sidelobe ratio in $A B\left(20\right.$ times the $\log _{10}$ of the sidelobe ratio). Several of Harris' results ${ }^{13}$ are collected in Table I and displayed in Figure 2; since Harris concentrated on weighting functions with very low sideiobes, we have extended his calculations to include weighting functions with sidelobes - 10 percent of the main lobe.

The weighting functions in Figure 2 fall in three groups. First, functions $(a, b)$ which are flat in the center and fall smoothly to zero at the edges have the poorest resolution for a given sidelobe ratio. The shape of the roll off-Gaussian or cosine-seems to have little effect. Second, several functions $(c, d, e)$ without variable parameters can be found which have 1-10 percent sidelobes but better resolution than the first group. Finally, the third set includes functions $(f, g, h)$ which are theoretically optimal for mainlobe width versus sidelobe ratio by different measures. 13,14 For sidelobe ratios in the .1 to .01 range these weighting functions are equivalent.

From this last set we select the more familiar Gaussian weights and choose the Gaussian function width equal to $5 / 8$ times the data range. This gives $f=1.6$ and sidelobes equal to 3 percent of the 
mainline. Figures $3(a)$ and $3(b)$ illustrate the taper-and-transform results for the sine wave test spectrum using this weighting. The sidelobes will double while the mainlobe only narrows by 10 percent if we choose a Gaussian width equal to $3 / 4$ of the data range.

With the resolution relation, equation 5 , we can look forward to difficulties with real measurements. With the longest ARPEFS measurement range reported to date ${ }^{9}, \Delta k=6.5 A^{-1}-$ rad., the pathlength resolution will be $\Delta r=1.55 \AA$. Nearest neighbor scattering atoms in that study appeared at path lengthis of $1.96 \AA, 3.2 \AA$, and 4.46 A-these peaks cannot be resolved with taper-and-transform Fourier analysis.

IV. THE AUTOREGRESSIVE PREDICTION METHOD

The taper-and-transform Fourier method produces a Fourier spectrum of our signal only after we taper the signal toward zero at the edges of the observation interval. Beyond the observation interval this method therefore arbitrarily (albeit implicitly) assigns zero as the signal value, contrary to any reasonable expectation based on the sequence measured. In fact, most arbitrary choices for the signal in this region could be characterized as "unreasonable". This is another way of saying that we do not want the fourier transform of our measured signal; we want the fourier transform of a signal of which we have only a short segment. Proper selection of a weignting function can minimize the problems of a short data range, but this 
does not address the underlying problem.

The autoregressive (AR) prediction approach to fourier analysis proceeds with different assumptions about the data analysis problem. $1,3,10$ In the AR method we assume that the data in the (limited) data range represent a few observations of an autoregressive process. By least-squares fitting these data we determine the process parameters and solve for the Fourier spectrum of the process. Because the range of the AR process is not limited to the observation interval, much better resolution is possible.

In an autoregressive process each data value, $x_{p}$, can be expressed as a linear combination of previous values,

$$
x_{p}=-\sum_{q=1}^{m} a_{q} x_{p-q}
$$

The number $m$ is called the "order" of the process; the coefficients $a_{q}$ constitute an autoregressive filter. In modeling a data sequence with an AR process, a set of coefficients $a_{q}$ and an order m must be calculated which can "predict" all the members of the data sequence. With the order less than the number of data points, the forward predictions in equation (6) and the backward predictions,

$$
x_{p-m}=-\sum_{q=1}^{m} a_{q} x_{p-m+q}
$$

form an overdetermined set of equations for the AR coefficients. The 
struciure of this set of equations is unusual since the autoregressive process employs data values to construct other data values. 10

For $n$ data values, we define a $2 n-2 m$ length vector, $\vec{b}$, containing the values from the left hand side of equations 6 and 7 . Similarly, $m$ AR coefficients form a vector, $\vec{a}$, and $a(2 n-2 m)$ by $m$ matrix, $\underset{\sim}{x}$, is constructed from the staggered data values as indicated on the right hand side. Then the least-squares equation for $a$ is

$$
\underset{\sim}{X} \vec{a}=-\vec{b}
$$

(see also ref. 10, page 249). These equations may be solved by fast recursive methods, ${ }^{15}$ but, for the signal-to-noise ratios encountered in the analysis of extended fine structure, we find that the Singular Value Decomposition (SVD) method ${ }^{16}$ for solving these equations to be more useful. As described in Ref. 17, the Singular Value Decomposition of a matrix $\underset{\sim}{x}$ gives

$$
\underline{x}_{2(n-m) \times m}=\underline{U}_{2}(n-m) \times 2(n-m) \sim 2(n-m) \times m v_{m \times m}^{\top}
$$

where $\underset{U}{U}$ and $\underline{V}$ are orthogonal matrices and $\underline{S}$ is a diagonal matrix whose entries may be ordered by size. The value of this approach to solving least-squares problems has been discussed in detail by Lawson and Hanson, 17 and the application of spectral estimation is discussed by Tufts and Kumaresan. ${ }^{16}$ Essentially, the SVo concentrates the significant signal content in the equations for the largest singular values. Then, when the solution for the original least-squares problem is constructed, only the largest singular values are used and 
the remainder-those associated with noise-are discarded. Thus the solution for the AR coefficients is written

$$
\vec{a}=-\sum_{i=1}^{p} \frac{\vec{v}_{i}}{\sigma_{i}^{2}}\left(U^{\top} \vec{b}\right)_{i}
$$

where $\vec{v}_{i}$ is the $i^{\text {th }}$ row of $\underline{y}$ and $\sigma_{i}^{2}$ is the $i^{\text {th }}$ singular value.

The appropriate number of singular values, $p$, may be selected by visual inspection. Since the SVO of random numbers will be random, the ordered singular values with fall with a constant slope unless they contain information (see Figure 8, as discussed below). With the highly overdetermined AR system of equations, we can always see a section of constant slope for high index singular values: when the singular values rise above this slope, they contain information.

An AR process of order $m$ has a Fourier power spectrum proportional to

$$
\frac{1}{1+\left.\sum_{q=1}^{m} a_{q} e^{-2 \pi i q p / N}\right|^{2}}
$$

Thus, one route to high resolution Fourier analysis proceeds as follows:

1. set $a_{0}=1$,

2. construct the least-squares equations and apply the SVD, 
3. select the number of singular values and solve for $a_{q}$, $q=1, m$ with equation 10 ,

4. set $a_{q}=0$ for $q=M+1, q_{\max }$ where $q_{\max }$ is a large power of 2, e.g. $q_{\max }=2048$,

5. fast Fourier transform the full sequence $\left[a_{q}\right]$, and

6. invert the square modulus of the transform.

While this approach has the greatest potential resolution, it is difficult to apply to real data. The resulting peaks are all very sharp, making it difficult to distinguish spurious from real hidden peaks. The peaks are strong functions of the order chosen and of the signal-to-noise ratio in the data. Furthermore, only the power spectrum is retrieved; the phase information is not available.

For these reasons we have adopted a more conservative approach, suggested by reference 1, which sacrifices some resolution in favor of greatly enhanced reliability and control. This procedure is:

1. set $a_{0}=1$,

2. construct the least-squares equations and apply the SVD,

3. select the number of singular values and solve for $a_{q}$, $q=1$, m with equation 10 ,

4. Use equation 6 to extrapolate the data sequence forward,

5. use equation 7 to extrapolate backward,

6. multiply the resulting sequence by a weighting function,

7. add zero value channels until the total number of channels is a large power of 2, e.g. 2048, and 


\section{8. fast Fourier transform the long sequence.}

The Fourier coefficients deriyed from this procedure can be further analyzed with the usual Hilbert back transformation. ${ }^{3,4}$ We have usually chosen an order equal to one half the number of data points, and we can typically extrapolate for approximately as many data points forward and backward as we originally measured.

The inherent control of this procedure comes in the examination of the extrapolated sequence. At some point in the extrapolation the new values begin to increase rapidly in amplitude and/or noise content (Fig. 3c). By placing the edge of our taper window at these points the unstable part of the extrapolation is eliminated. Furthermore, the window weights the extrapolated points significantly less than the real data values, moderating the effect of the new values on the final spectrum. This last advantage is crucial for practical spectroscopic signals which are not exact sinusoids.

An example of the extrapolation is shown in Figure $3 c$, and its effect on the fourier spectrum is shown in figure 3d. The signal in Figure la was fitted to an AR process of order 64 and 4 singular values were required (as expected for 2 real sinusoids ${ }^{16}$ ). Extrapolation gives Figure $3 c$. Figure $3 d$ dramatically illustrates the potential of this method for increasing resolution in Fourier analysis.

At this point it is useful to note that the ARP-Fourier transform method is not a "deconvolution" of the data which can produce spurious peaks through unreliable resolution enhancement. As we illustrated in 
Figure 4, our net process solves a problem with the taper-and-transform Furier method. In Figure $4 a$ it is obvious on visual inspection that more than one frequency is present, but the Fourier transform will have Gibb's oscillations. When the taper (weighting function) is applied as in Fig. $3 b$, the beat structure is lost while the Gibb's oscillations in the Fourier transform are suppressed (Figure $3(b))$. From this perspective the unadorned Fourier transform and the taper-and-transform process are clumsy operations that obscure the frequency information inherent in the data. When the ARP is applied, Figure $4(c)$, data on the ends of the measurement are no longer lost when the window function is applied, Figure $4(d)$.

Our overall procedure requires three parameters: the number of singular values, the number of AR coefficients, and the final taper width. As discussed above and in reference 17, the number of singular values may be determined by inspection. For poor signal-to-noise conditions, the size and variability of the singular values associated with noise will make this choice more difficult. Autoregressive orders between $N / 2$ and $3 N / 4$ are are recommended by Tufts and Kumaresan. ${ }^{16}$ Our choice of a taper width at just less than twice the measured data wioth reduces the importance of our choice in the first two parameters.

Up to this point we have assumed that our measurement can be successfully approximated by an autoregressive process. In reexamining this point we divide the question in two parts: i) how closely can a cosinusoidal series be represented by an autoregressive 
process, and $i$ ) how closely does a cosinusoidal model fit ARPEFS data? For the first part we can note the discussion of Ulrych and $00 e^{10}$. Beginning with a finite difference equation for a sinusoidal series, they demonstrate that such a series can be represented by a combination autoregressive, moving average (ARMA) model; they also show that such an ARMA model can be represented by an infinite order pure autoregressive model. Numerical work by Tufts and Kumaresan 16 supports the conclusion that AR models can represent sinusoidal series; their method can give resolution near the theoretical limit even for low signal-to-noise ratios.

The second question is more difficult to address, but it impacts every method of harmonic analysis applied to ARPEFS. Specifically, if the cosine form breaks down, the taper-and-transform approach will fail as the autoregressive approach does. We will examine some of the possible problems in the next section.

\section{DISCUSSION}

Two important features neglected in the sine wave model spectrum are the amplitude and phase variation with $k$ in real ARPEFS data. The sine wave model spectrum neglected any variation in frequensy due to nonlinearity in $b_{j}$ and any variation in amplitude due to $\left|f\left(a_{j}\right)\right| \exp \left[-\sigma^{2} k^{2}\left(1-\cos a_{j}\right)-L_{j} / \lambda k\right]$.

To examine a model containing realistic amplitude and phase functions on a scale similar to our data we have generated a spectrum 
by adding noise to

$$
\begin{aligned}
& x(k)=\cos \left(173^{\circ}\right)\left|f\left(173^{\circ}\right)\right| \cos \left[4.46 k+\phi\left(173^{\circ}\right)\right] e^{-.02 k^{2}-5.3 / k} \\
& +2 \cos \left(116^{\circ}\right)\left|f\left(116^{\circ}\right)\right| \cos \left[3.21 k+\phi\left(176^{\circ}\right)\right] e^{-.014 k^{2}-5.3 / k},
\end{aligned}
$$

where $f$ and $\phi$ are derived from summed partial-wave phase shifts. 18 Direct application of the AR prediction gives the result in Figure $5 \mathrm{a}$ and the Fourier transform in Figure 5b. The increase in amplituce at low $k$ in the linear prediction is a consequence of the amplitude structure for scattering through $116^{\circ}:\left|f\left(116^{\circ}\right)\right|$ peaks at - $5 A^{-1}-$ rad as shown in Figure 6. The AR method presumes that this is a rising signal and continues the trend to lower $k$. At higher $k$, the AR method tries to force this single decayiny frequency to be modeled by infinite sine waves: it must sum two nearby frequencies to simulate the amplitude decline. The Fourier spectrum then contains a split peak for this scattering event.

The rising low $k$ amplitude effect can be recognized in the predicted spectrum and remedied by analyzing $k x(k)$. The $k$ weighting helps to cancel the decline of $\left|f\left(a_{j}\right)\right|$ at higher $k$ and has been used extensively for analys is of EXAFS data. ${ }^{5}$ This weighting evens out the 1 inear prediction shown in Figure $5 \mathrm{c}$, and the resulting Fourier transform amplitudes (Fig. 5d) are more similar to the average amplitudes of the signals within the real measurement range. Whatever weighting is employed, the important sefaration of the 
Fourier frequencies is still effected by the autoregressive prediction followed by Fourier transformation. The amplitude variation places an upper limit on the resolution obtainable from the AR analysis of real data. When the amplitude function falls with the same shape as the beat envelop, then the AR analysis cannot distinguish between them. Variation of the frequency with $k$ violates the stationary assumption in the application of the autoregressive model. Thus ARPEFS peaks with phase functions strongly nonlinear in $k$ will be modeled incorrectly, probably being represented as more linear than they really are. If the phase has an average slope at the beginning of the data range which is different from its average slope at the end, the extrapolation procedure sometimes yields a sligntly doubled or asymmetric peak which must not be mistaken for two.

The frequency variation may also explain the empirical selection of a large process order $\mathrm{m}$. In the usual application of the AR technique ${ }^{10}$ the order is chosen by some criterion based on the prediction error; that is, the difference between the linear prediction and the data values. While this criterion can give a prediction filter for pure sinusoids in the presence of noise, valid for inf inite range, we seek an adequate representation of a more complex oscillating signal over a small range. Our signal does not result from any autoregressive process, and a large order may medel nuances of nonlinear phase and noise.

The impact of modelling this non-stationary signal with an 
autoregressive filter is minimized because we do not rely on the Fourier spectrum itself for the final analysis. Following Martens, 4 we apply a Hilbert transformation ${ }^{3}$ to our data. From the complex exponential form of the cosine

$$
A_{j} \cos \left(p_{j} k+\phi_{j}\right)=\frac{A_{j}}{2} e^{i\left(p_{j} k+\phi_{j}\right)}+\frac{A_{j}}{2} e^{-i\left(p_{j} k+\phi_{j}\right)}
$$

we see that the transform of the cosine is real and peaked near $p_{j}$ and $-p_{j}$. By using only the positive frequency components, a complex back transform gives

$$
\frac{A_{j}}{2} e^{i\left(p_{j} k+\phi_{j}\right)}=\frac{A_{j}}{2} \cos \left(p_{j} k+\phi_{j}\right)+\frac{i A_{j}}{2} \sin \left(p_{j} k+\phi_{j}\right)
$$

The amplitude and phase functions of the original cosine wave can be derived as the amplitude and phase of this complex sequence. For our signal the actual cosine argument is $k\left(r_{j}-r_{j}\right)+\phi_{j}$, so we subtract the potential phase shift, $\phi_{j}$, and fit the resulting sequence to a line. The slope of this line gives the averaged geometrical position we seek. The crucial point is this: we only use the cosine phase function in the region of $k$ where we made actual measurements. Thus the entire AR prediction fourier analysis serves only to isolate a single frequency. The position and amplitude of the Fourier peaks need not be accurate for us to obtain accurate geometries.

This complex backtransformation procedure does introduce one important source of error: we lose the wings of the Fourier peak 
spectrum for the oscillation we isolate. This implies that the non-linear phase information and strong amplitude dependence of the ARPEFS Oscillation will be missing in the backtransformed peak: we necessarily derive an averaged frequency and smoothed amplitude dependence. While not ideal, this result is certainly preferred to mixing the arguments of two different cosine oscillations.

Since there are a large number of variable parameters in even this simple model, we cannot yet give a complete analysis of the effects of background subtraction and signal/noise ratio. Generally, the AR prediction produces a "peakier" spectrum than one might imagine being correct. 1,10 Thus, errors in background subtraction appear as small peaks at harmless low $r_{j}$ values. When the beat pattern of two peaks approaches the width of the actual measurement range, then errors in background subtraction may interfere with resolution. Signal/noise ratios greater than two al low approximately double the resolution of the taper approach, with errors in geometry of $<0.02$ A. Errors increase rapidly for signal/noise ratios failing below 1. Until more experience is acquired with the AR method, prudence suggests examination of these effects for model spectra closely mimicking the actual data before assigning error limits.

As a practical example of the improved analysis of ARPEFS data, we have analyzed ${ }^{9}$ the modulations (Figure $7 \mathrm{a}$ ) in the sulfur is photoemission intensity emitted along the [110] direction from a $c(2 \times 2) S / N i(100)$ adsorbate system. The fourier transform via the taper approach shows distinct peaks (Figure $7 \mathrm{~b}$ ), but each peak is an average 
of several path-length differences. The singular values for the application of an 128 order AR prediction are shown in Figure 8. The slope of the singular values is roughly constant-as indicated by the plotted derivative--above singular value 17 . Thus 17 principal vectors were used to construct the AR filter. The AR prediction is shown in Figure $7 c$, and the Fourier transform gives Figure $7 d$. Now the individual peaks are clearly separated and they can be assigned to scattering path-length differences. ${ }^{9}$

\section{SUMMARY}

Autoregressive prediction provides a method for greatly increasing the resolution of Fourier analysis of sinusoidal data. Using the extrapolate-taper-transform method described here, we can always do as well or better than the taper-transform approach. If the signal/noise ratio is so poor that the extrapolation fails immediately, then the AR procedure reverts to the usual taper method. For all other cases the resolution is improved. Furthermore, the method is easy to implement, computationally efficient, and controllable.

The resolution improvement afforded by the autoregressive prediction method scales with the quality of the experimental measurements. Low precision or widely spaced measurements do not contain enough information to accurately detemine the autoregressive coefficients. Our moderate precision measurements yield moderate precision autoregressive coefficients; our coefficients allow successful extra- 
polation as we have demonstrated, but they are not precise enough for the analytic power spectrum formula.

Two improvements in the application of autoregressive prediction to spectroscopic data require further investigation. First, the statistical accuracy of the data values can vary significanily across a spectrum; the least-squares fit of the autoregressive coefficients should be weighted accordingly. Second, the autoregressive method assumes equal intervals between measurements; for ARPEFS we do not have equally spaced data. This problem is more difficult: the AR process given in equation (6) steps by a single fixed amount. However, there snould be some AR process whose fourier spectrum closely approximates the Fourier specrum of our data even if our measurements do not fall on an even mesh. Such questions are being examined in the signal processing literature, e.g., references 20 and 21, and new methods should be available soon.

Our final procedure is empirical for the same reasons the familiar taper-and-transform method is empirical. Ideal frequency analysis-the separation of our signal into each component oscillation--cannot be accomplished with noisy, finite-range measurements. Furthermore, harmonic analysis is only approximately valid for our spectroscopy: nonlinear phase shifts and energy-dependent scattering power preclude pure sine-wave signals. The procedure we have , described here will, however, give a useful, high-resolution Fourier transform from real spectroscopic signals.

Formulation of the autoregressive prediction method from the 
vantage of information theory has led to its description as maximum entropy spectral analysis. $7,8,10,22$ Faced with the problem of estimating the fourier transformation of an oscillatory signal given only a short measurement range, the autoregressive method fits a general oscillatory model to the measurements. The resulting overdetermined set of equations are reduced by maximizing the entropy of the model. Thus, of all the possible models which give the same leastsquares error, we select the model which adds the least new information, i.e. the one with the most signal entropy.

Data analysis methods can generally be compared by examining the information they add to the measurement. The AR method assumes that the data represent a process whose Fourier spectrum does not change outside the data sequence: it attempts to add no new inf ormation. 7,8,10 The taper-and-transf orm approach added the "information" that the signal was zero where it was not measured; this is contrary to any reasonable expectation. Directly fitting the data. to a model of the physical process (eqn. (1)) would be the ultimate addition of information, but small uncertainties in the measurement and in the model usually prevent this approach ${ }^{5}$ from being successful.

Note that extrapolation after direct physical model fitting has a different meaning than our AR prediction. Extrapolating by evaluating a physical model estimates a physical signal. The AR extrapolation does not estimate a physical signal; instead it reflects the frequency content over the original measured interval. The AR prediction 
estimates an autoregressive model, not a physical one. We are not attempting to predict a measurable quantity; the extrapolation is merely one step in a harmonic analysis of our data.

Finally we note that this conservative approach to AR Fourier analysis can also be applied to a number of spectroscopic problems. Extended $X$-ray Absorption Fine Structure (EXAFS) has a nearly identical form to eq. (1), and the autoregressive prediction would allow high resolution Fourier analysis of more general utility than the beat method of Martens. 19 Many problems in spectroscopic deconvolution via the Fourier transform can also benefit from this $A R$ approach. Direct AR power spectral analysis has been successfully applied to this problem, ${ }^{23}$ but the danger of spurious peaks is particularly acute when we are seeking resolution ennancement. An extrapolation-taper procedure would allow a more controlled, albeit more moderate resolution enhancement. 


\section{Acknowledgements}

The authors are grateful for instructive discussions with J. D. Klein and 5. W. Robey.

This work was supported by the Director, Office of Energy Research, Office of Basic Energy Sciences, Chemical Sciences Division of the U.S. Department of Energy under Contract No. DE-ACO3-76SF00098. It was performed at the Stanford Synchrotron Radiation Laboratory, which is supported by the Department of Energy, Office of Basic Energy Sciences and the National Science Foundation, Division of Materials Research. 


\section{Ref erences}

1. S. M. Kay, S. L. Marple, Jr., Proc. IEEE 69, 1380 (1981).

2. E. O. Brigham, The Fast Fourier Transform, Prentice-Hall, Englewood Cliffs, N.J., 1974.

3. J. F. Claerbout, Fundamentals of Geophysical Data Processing, McGraw-Hill, New York, 1976.

4. G. Martens, P. Rabe, N. Schwentner, and A. Werner, Phys. Rev. B 17. 1481 (1978).

5. P. A. Lee, P. A. Citrin, P. Eisenderger, B. M. Kincaid, Rev. Mod. Phys. 53, 769 (1981).

6. 2. Hussain, D. A. Shirley, C. H. Li, S. Y. Tong, Proc. Natl. Acad. Sci. USA 78, 5293 (1981).

7. E. T. Jaynes, Proc. IEEE 70,939 (1982).

8. J.A. Cadzow, ProC. IEEE 70, 907 (1982).

9. J. J. Barton, C. C. Bahr, Z. Hussain, S. W. Robey, J. G. Tobin, L. E. Klebanoff, and D. A. Shirley, Phys. Rev. Lett. 51, 272 (1983).

10. S. Haykin, ed. Nonlinear Methods of Spectral Analysis, Topics in Applied Physics, v. 34, Springer-Verlag, Berlin, 1983.

11. Derived by analogy with P. A. Lee and J. B. Pendry, Phys. Rev. B 11. 2795 (1975).

12. We define our signal-to-noise ratio as the ratio of the root mean square (rms) signal power divided by the rms noise power. For a sine wave with amplitude $A$, the rms signal level is $0.707 \mathrm{~A}$; the rms noise power equals the standard deviation of the noise, $\sigma$. The signal-to-noise ratio for a sine wave in noise is 
then equal to $0.707 \mathrm{~A} / \sigma$. The idealized Fourier spectrum for a sine wave in noise would be a single peak of height $A / 2+\sigma / \sqrt{N}$ with a flat background level equal to $\sigma / \sqrt{\mathrm{N}}$.

13. F. J. Harris, Proc. IEEE 66, 51 (1978).

14. A. H. Nuttall, IEEE Trans. Acoust. Speech and Signal Proc., ASSP-29, 84 (1981).

15. L. Marple, IEEE Trans. Acoust. Speech, Signal Process. vol. ASSP-28, 441 (1980). This reference reports a FORTRAN program to calculate the AR coefficients.

16. D. W. Tufts and R. Kumaresan, Proc. IEEE, $\underline{70}, 975$ (1982).

17. C. L. Lawson, R. J. Hanson, Solving Least-Squares Problems, Prentice-Hall, Englewood Cliffs, NJ (1979). The computer programs published in this reference are available commercially from IMSL, NBC Building, 7500 Bellaire Bivd., Houston, Texas 77036.

18. C. S. Fadley, private communication.

19. G. Martens, P. Rabe, N. Schwentner, A. Werner, Phys. Rev. Lett. 39, 1411 (1977).

20. J. P. Schott and J. H. McClellan, IEEE Trans. on Acoust., Speech, Signal Processing, vol. ASSP 32, 410 (1984).

21. T. A. Ramstad, IEEE Trans. on Acoust., Speech, Signal Processing, vol. ASSP 32, 577 (1984).

22. A. Van den Bos, IEEE Trans. on Information Theory, IT-17, 493 (1971).

23. R. P. Vasquez, J. D. Klein, J. J. Barton, F. J. Grunthaner, J. Electron Spectrosc. Relat. Phenom. 23, 63 (1981). 
Table I.

Resolution factors and sidelobe ratios for Fourier weighting functions. For a data range of $\Delta k$, the full width of the Foulier amplitude mainlobe for these weighting functions is $\Delta R$ where $\Delta$ rak $=2 \pi f$. The ratio of the maximum sidelobe peak value to mainlobe peak is $\mathrm{SL}$. These results are displayed in Figure 2.

\begin{tabular}{|c|c|c|c|c|c|}
\hline $\begin{array}{l}\text { Curve in } \\
\text { Figure } 2 \\
\end{array}$ & $\begin{array}{l}\text { Weighting } \\
\text { Function }\end{array}$ & $\begin{array}{l}\text { Formu la } \\
h=\Delta k / 2\end{array}$ & B & $f$ & SL \\
\hline & None & $w(x)=1$ & & 1.20 & .22 \\
\hline (a) & $\begin{array}{l}\text { Tukey: } 10 \\
\text { for } B=0 \\
\text { Hanning }\end{array}$ & $\begin{array}{l}\text { for }|x-h|<B h \quad w(x)=1 \\
\text { for } x-h>B h \quad w(x)= \\
\frac{1}{2}-\frac{1}{2} \cos \left[n\left(\frac{x-h-h}{8 h}\right)\right]\end{array}$ & $\begin{array}{l}.75 \\
.66 \\
.50 \\
.33 \\
.25 \\
.00\end{array}$ & $\begin{array}{l}1.38 \\
1.43 \\
1.57 \\
1.72 \\
1.80 \\
2.00\end{array}$ & $\begin{array}{l}.21 \\
.20 \\
.18 \\
.13 \\
.11 \\
.03\end{array}$ \\
\hline (b) & $\begin{array}{l}\text { Gaussian Step, } \\
\text { or } \\
\text { Error function }\end{array}$ & {$\left[\frac{1}{2}+\frac{1}{2} \operatorname{erf}\left(\frac{x-B h}{\sqrt{2} B h}\right)\right]\left[\frac{1}{2}-\frac{1}{2} \operatorname{erf}\left(\frac{2 h-B h-x}{\sqrt{2} B h}\right)\right]$} & $\begin{array}{l}.125 \\
.250 \\
.333 \\
.500 \\
.750\end{array}$ & $\begin{array}{l}1.37 \\
1.47 \\
1.72 \\
2.02 \\
2.18\end{array}$ & $\begin{array}{l}.21 \\
.18 \\
.14 \\
.04 \\
.02\end{array}$ \\
\hline (c) & Riesz 10 & $1.0-\left|\frac{x-h}{h}\right|^{2}$ & - & 1.59 & .09 \\
\hline (d) & Cosine 10 & $\cos \left[\left(\frac{x-h}{h}\right) \pi / 2\right]$ & - & 1.65 & .07 \\
\hline (e) & Riemann & {$\left[\sin \left(\frac{x-h}{h}\right) \pi\right] /\left(\frac{x-h}{h}\right) \pi$} & - & 1.74 & .05 \\
\hline
\end{tabular}


Table I continued.

\begin{tabular}{|c|c|c|c|c|c|}
\hline $\begin{array}{l}\text { Curve in } \\
\text { Figure } 2 \\
\end{array}$ & $\begin{array}{l}\text { Weighting } \\
\text { Function }\end{array}$ & $\begin{array}{l}\text { Formula } \\
h=\Delta k / 2\end{array}$ & B & $\mathbf{f}$ & SL \\
\hline (f) & Van Der Maas & $\begin{array}{l}\frac{B 1_{1}\left[B \sqrt{1-\left((x-h / h)^{2}\right.}\right]}{2 h \sqrt{1-((x-h) / h)^{2}}} \\
+\frac{1}{2} 6(x-2 h)+\frac{1}{2} \sigma(x)\end{array}$ & $\begin{array}{l}.5 \\
1.0 \\
2.0 \\
3.0 \\
3.5 \\
4.0 \\
5.0\end{array}$ & $\begin{array}{l}1.14 \\
1.17 \\
1.28 \\
1.38 \\
1.43 \\
1.51 \\
1.65\end{array}$ & $\begin{array}{l}.26 \\
.20 \\
.15 \\
.10 \\
.08 \\
.06 \\
.03\end{array}$ \\
\hline (g) & Gaussian & $\exp \left\{-\ln 2\left[\frac{2(x-h)}{B h}\right]^{2}\right\}$ & $\begin{array}{l}.80 \\
1.00 \\
1.24 \\
1.50 \\
2.0 \\
2.4 \\
3.0 \\
4.0\end{array}$ & $\begin{array}{l}2.22 \\
1.82 \\
1.58 \\
1.45 \\
1.33 \\
1.29 \\
1.26 \\
1.23\end{array}$ & $\begin{array}{l}.001 \\
.01 \\
.03 \\
.07 \\
.12 \\
.15 \\
.17 \\
.19\end{array}$ \\
\hline (h) & Kaiser-Bessel & $\frac{I_{0}\left[B \sqrt{\left.1-((x-h) / h)^{2}\right]}\right.}{2 h}$ & $\begin{array}{l}.5 \\
1.0 \\
1.5 \\
2.0 \\
3.0 \\
3.5 \\
4.0 \\
5.0\end{array}$ & $\begin{array}{l}1.21 \\
1.24 \\
1.29 \\
1.36 \\
1.50 \\
1.58 \\
1.65 \\
1.80\end{array}$ & $\begin{array}{l}.21 \\
.18 \\
.15 \\
.12 \\
.07 \\
.04 \\
.03 \\
.01\end{array}$ \\
\hline
\end{tabular}


Figure Captions

Figure 1. (a) Sum of two sine waves, periods of 5 and $6 A$, plus 10 percent pseudo-Gaussian noise. (D) Fourier amplitude of the sequence in $1(a)$. (c) Extension of the sine waves of (1a) by appending zeros. Set above the signal is a plot of the weighting window function; it has a baseline of zero and a height of one. (d) Fourier amplitude of Fig. I(c).

Figure 2. Resolution factor versus sidelobe-to-mainlobe ratio for several weighting functions. Abcissia is $f$ in $\Delta r \Delta k=2 \pi f$; for a data range of $6.3 A^{-1}$-rad., f will be the Fourier resolution in $A$. Ordinate is the maximum sidelobe peak value divided by the mainlobe peak. The plotted values are given in Table I. The point at $f=1.21$ and sidelobe $=.22$ represents an unweighted fourier transform. The weighting functions are given in Table I. (a) Tukey weighting, ref. 10, pg. 66. This function is flat in the center and rolls off as a cosine on the data extremes. (b) Gaussian Step or Error function. Similar to (a) but using a Gaussian roll-off. (c) Riesz polynomial, ref. 10, pg. 65. (d) Riemann weighting, ref. 10, pg. 65. (e) cosine weighting, ref. 10, pg. 60. (f) Van der Maas weighting, ref. 11, pg. 90. (g) Gaussian weighting, ref. 10, pg. 69, (h) Kaiser-Bessel weighting, ref. 11, pg. 89. 
Figure 3. (a) Extended sine wave from Fig. $1(c)$ and, set above, the weights used for taper-and-transform Fourier analysis. The base of the weighting function is zero and its peak is one. (b) Fourier transform of sine wave times weights from Fig. 3(a). (c) Autoregressive prediction of the signal in Fig. 1(a), using an order $m=64$, half of the 128 points. The new weights is set above. (d) Fourier amplitude of the product of the prediction results and weights from $F$ ig. $3(\mathrm{c})$.

Figure 4. Weighting function interaction with autoregressive prediction. (a) Test sequence of two sine waves and noise as in Fig. 1. Note the beat structure. (b) Data from (a) times Gaussian weights. Gaussian width is $5 / 8$ times the data range. Note the loss of beat structure. (c) AR prediction of the data in (a). (d) AR prediction from (c) times Gaussian weights. Gaussian width is $5 / 8$ times the extended data range. Note the reduced emphasis of the extrapolated region.

Figure 5. (a) Autoregressive prediction of a simulated signal from equation (9). (b) Fourier amplitude of Fig. 3(a) times Gaussian weights. (c) Autoregressive preaiction of $k$ times the simulated signal in Fig. 3(a). (d) Fourier amplitude of Fig. $3(\mathrm{c})$ times Gaussian weights. 
Figure 6. Magnitude of the scattering amplitude, $|f(a, k)|$, for Ni atom at $a=116^{\circ}$ and $a=173^{\circ}$. The mild amplitude behavior of the scattering for $173^{\circ}$ gives a simple fourier peak shape; the steep drop at high $k$ for scattering through $116^{\circ}$ leads to a doubled Fourier peak.

Figure 7. (a) Angle-resolved photoemission extended fine structure from $S(1 s) c(2 \times 2) S / N i(100)$ along [110]. The weighting function used for the taper is plotted offset above the data. Its minimum is zero and maximum is one. (b) Taperand-transform Fourier amplitude for (a). (c) Autoregressive prediction of (a). An order $M=64$ was used for 128 points interpolated from the raw data. The weighting function is set above as for (a). (d) Fourier amplitude of the product of the window and extrapolated data in $(c)$.

Figure 8. Selection of rank for the singular value decomposition for order 128 autoregressive $f i t$ to the data shown in figure 7. The singular value decomposition algorithm (ref. 17) automatically orders the singular values by size. The values $\sigma_{i}^{2}$, are plotted versus $i$ as solid circles connected by a thin line; their magnitude is given by the left hand axis. The point by point differences are plotted as the thick line with the right hand axis giving the scale. The rank is selected as the point where the singular values depart from constant slope. 


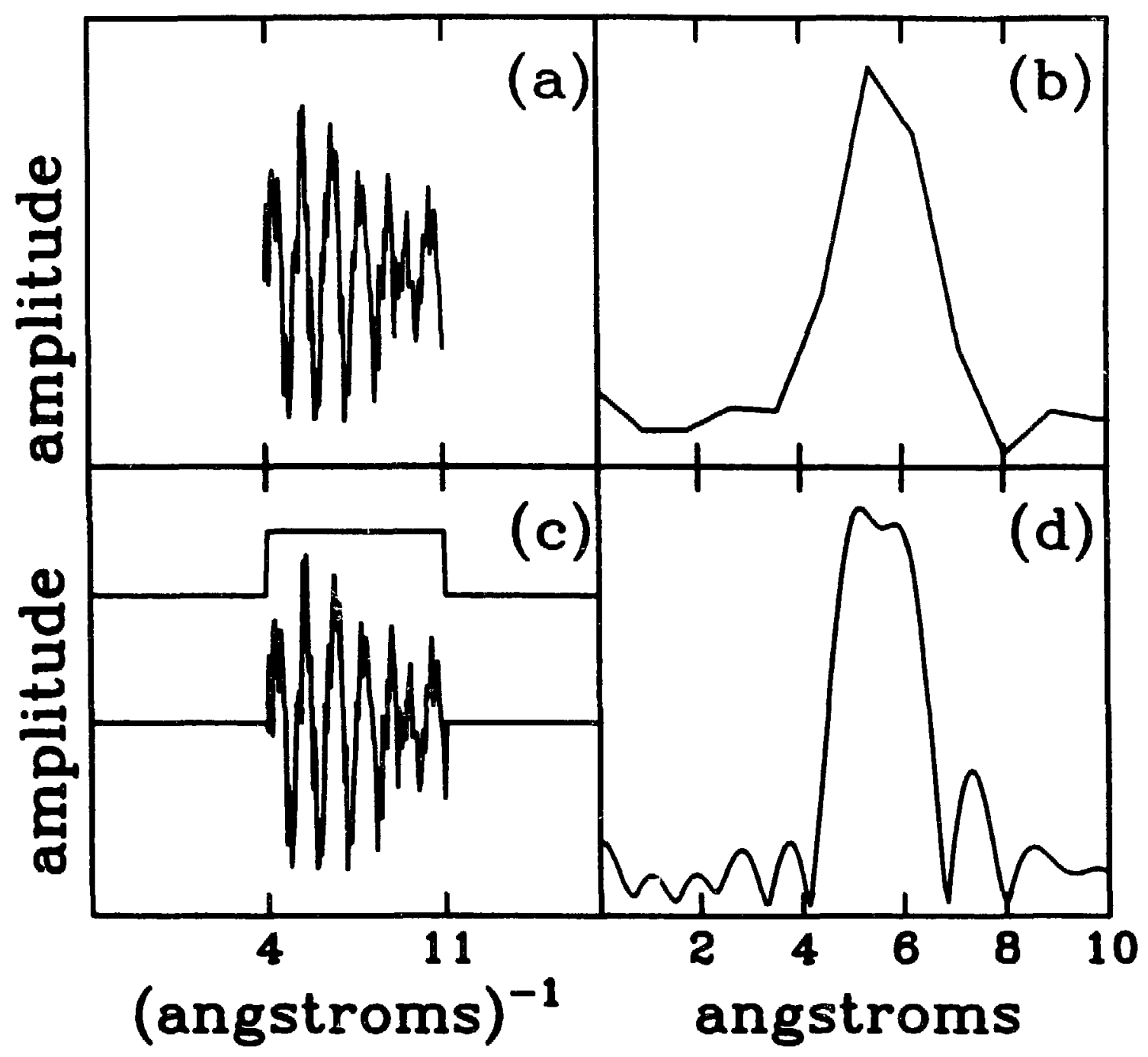

XBL 848-3552

Figure 1 


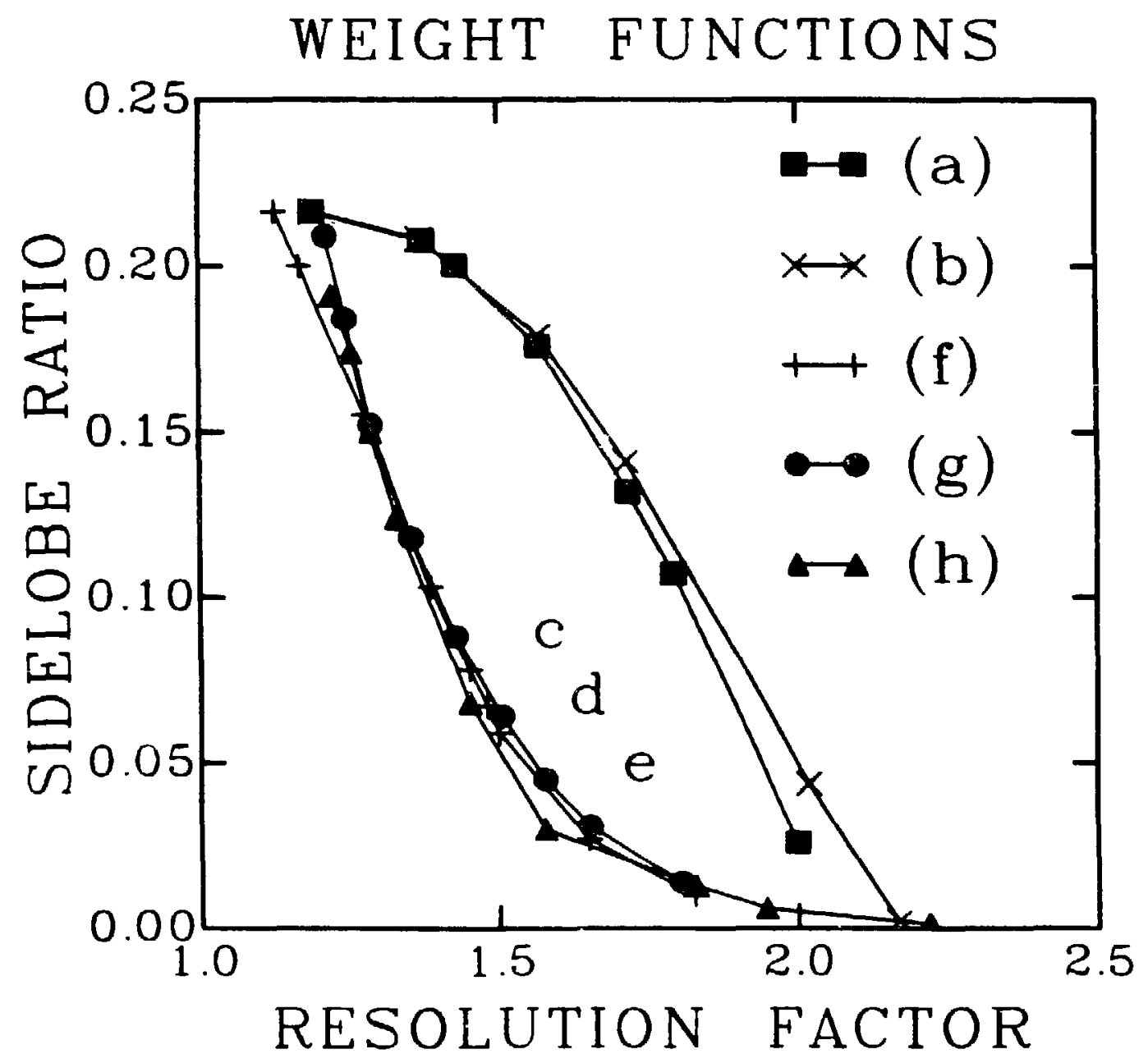

XBL 844-1349

Figure 2 


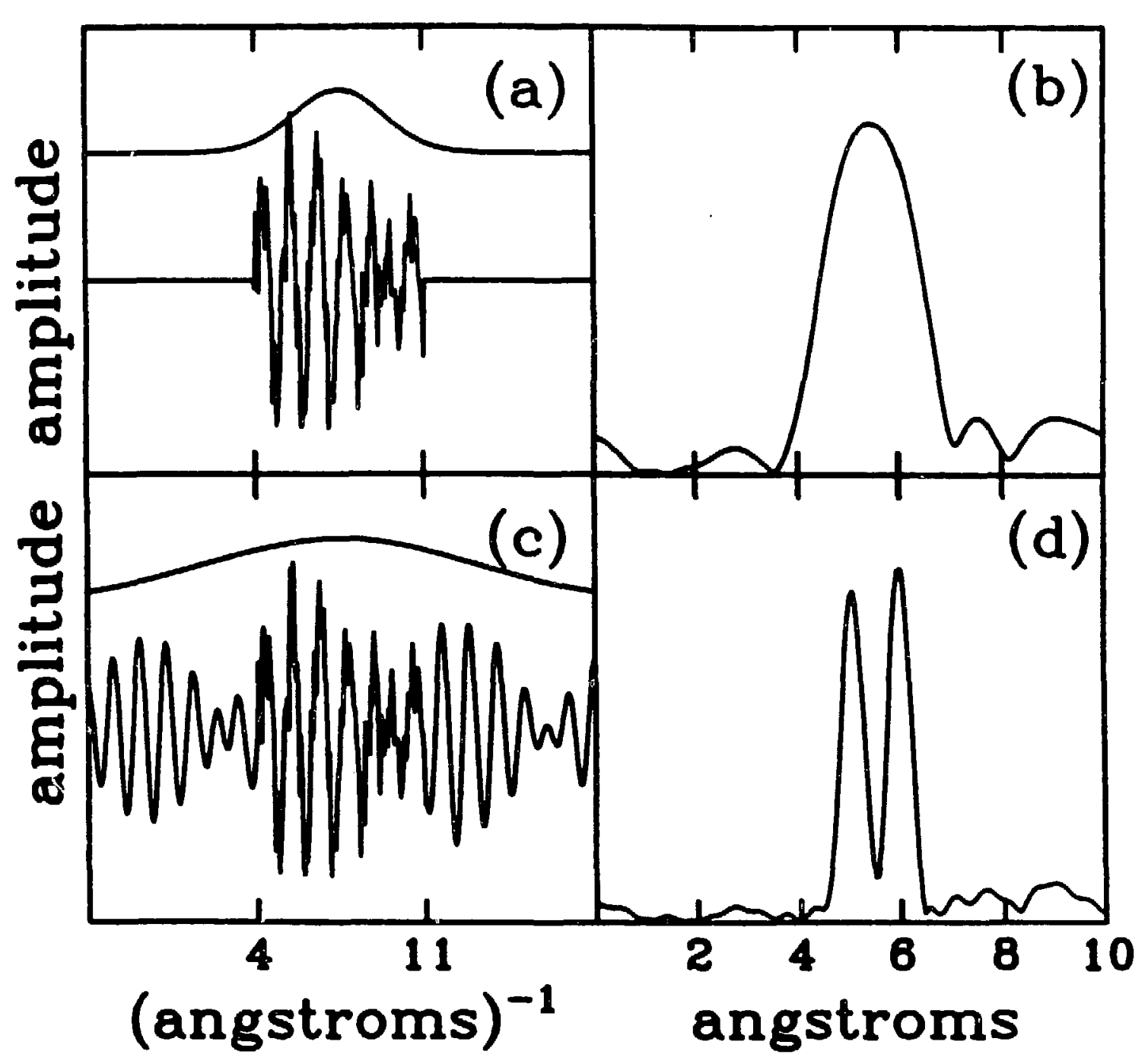

XBL 848-3553

Figure 3 


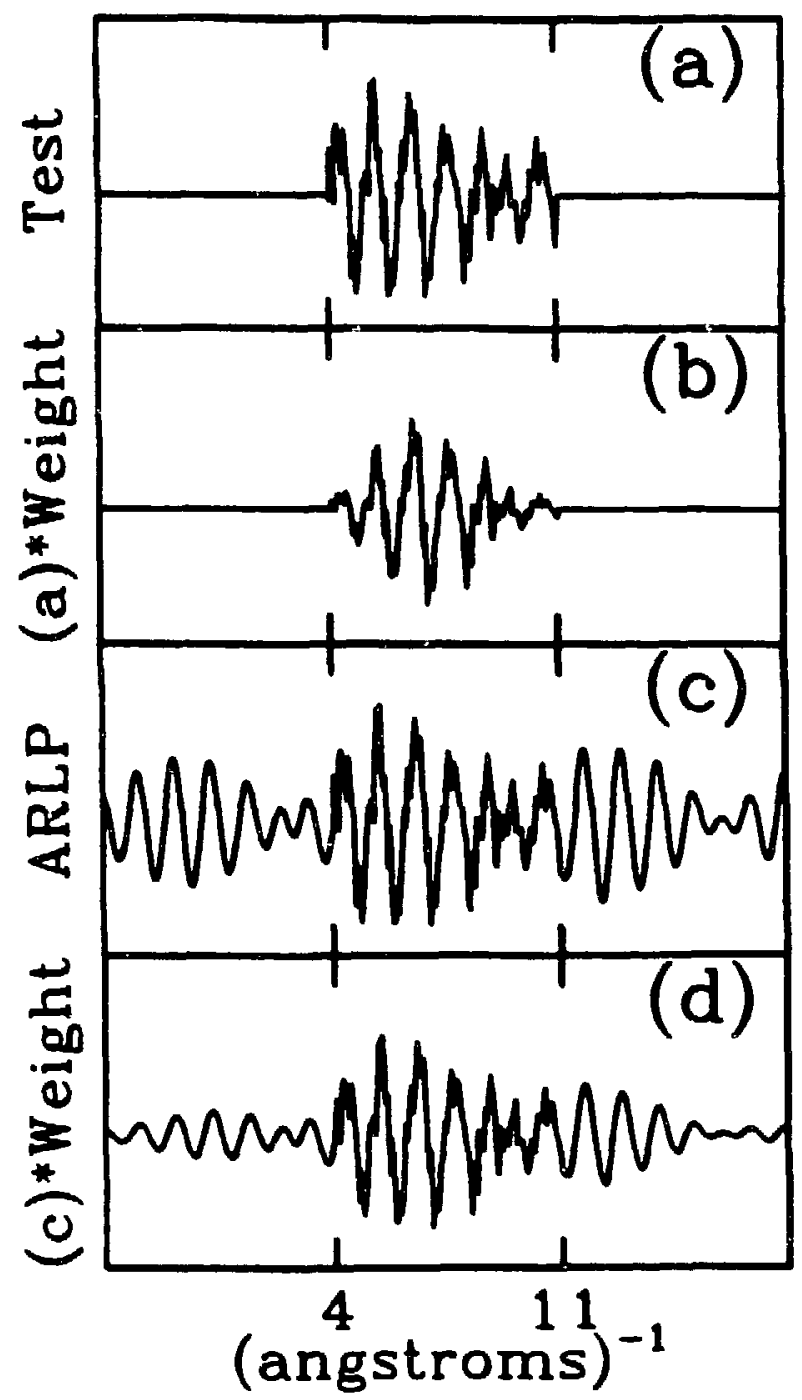

XBL $848-3550$

Figure 4 


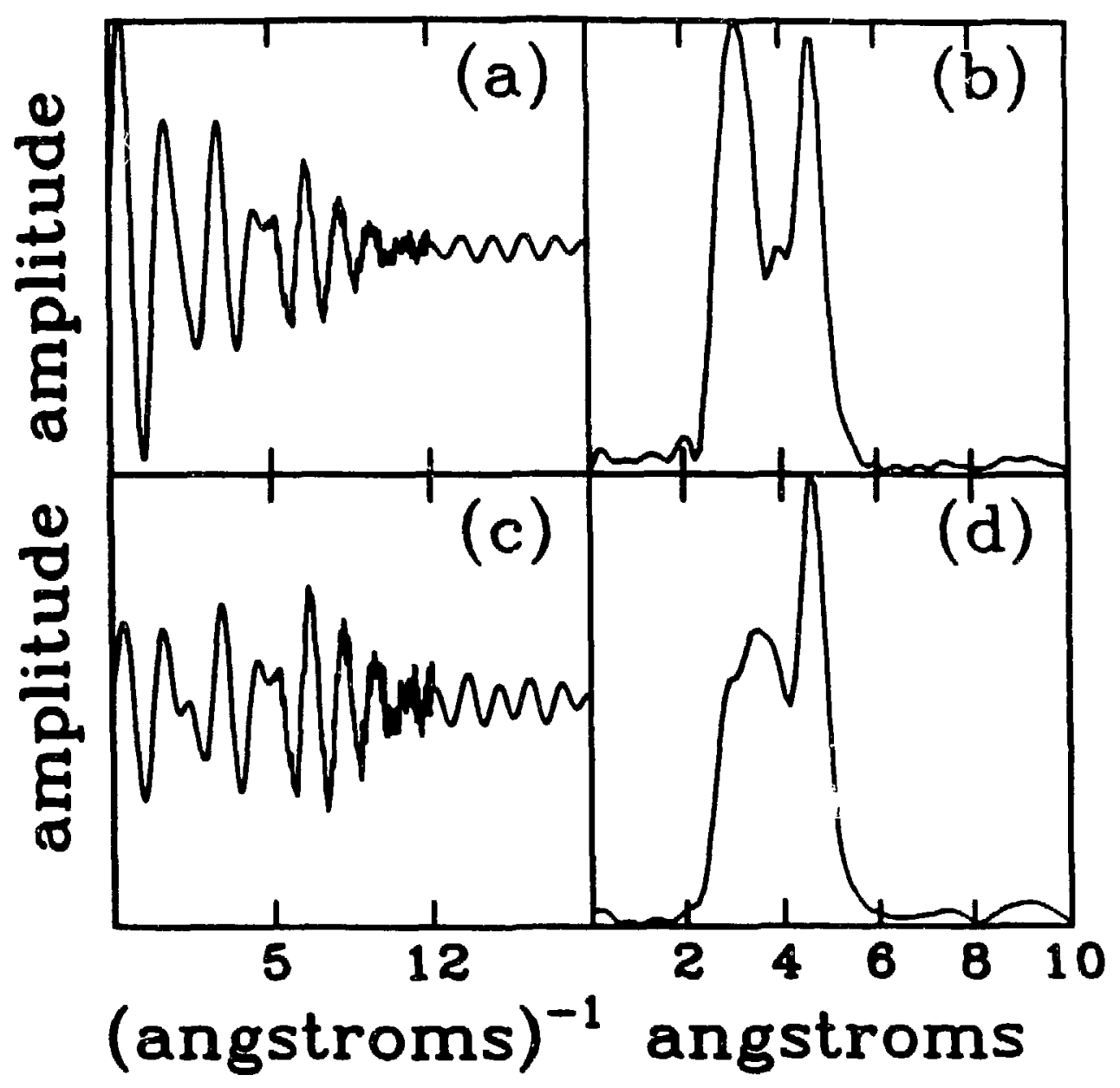

XBL 848-3551

Figure 5 


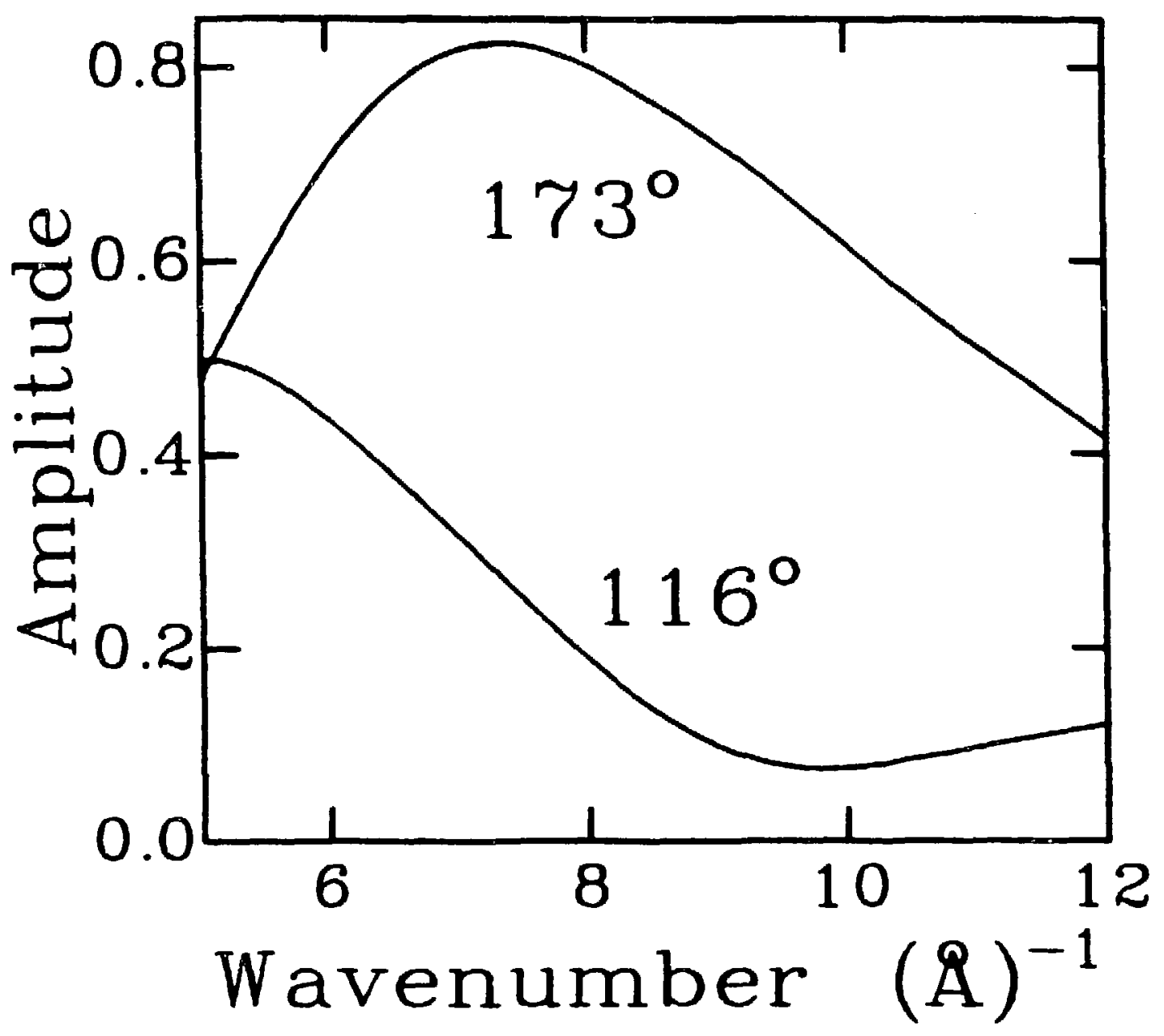

XBL $844-1350$

Figure 6 


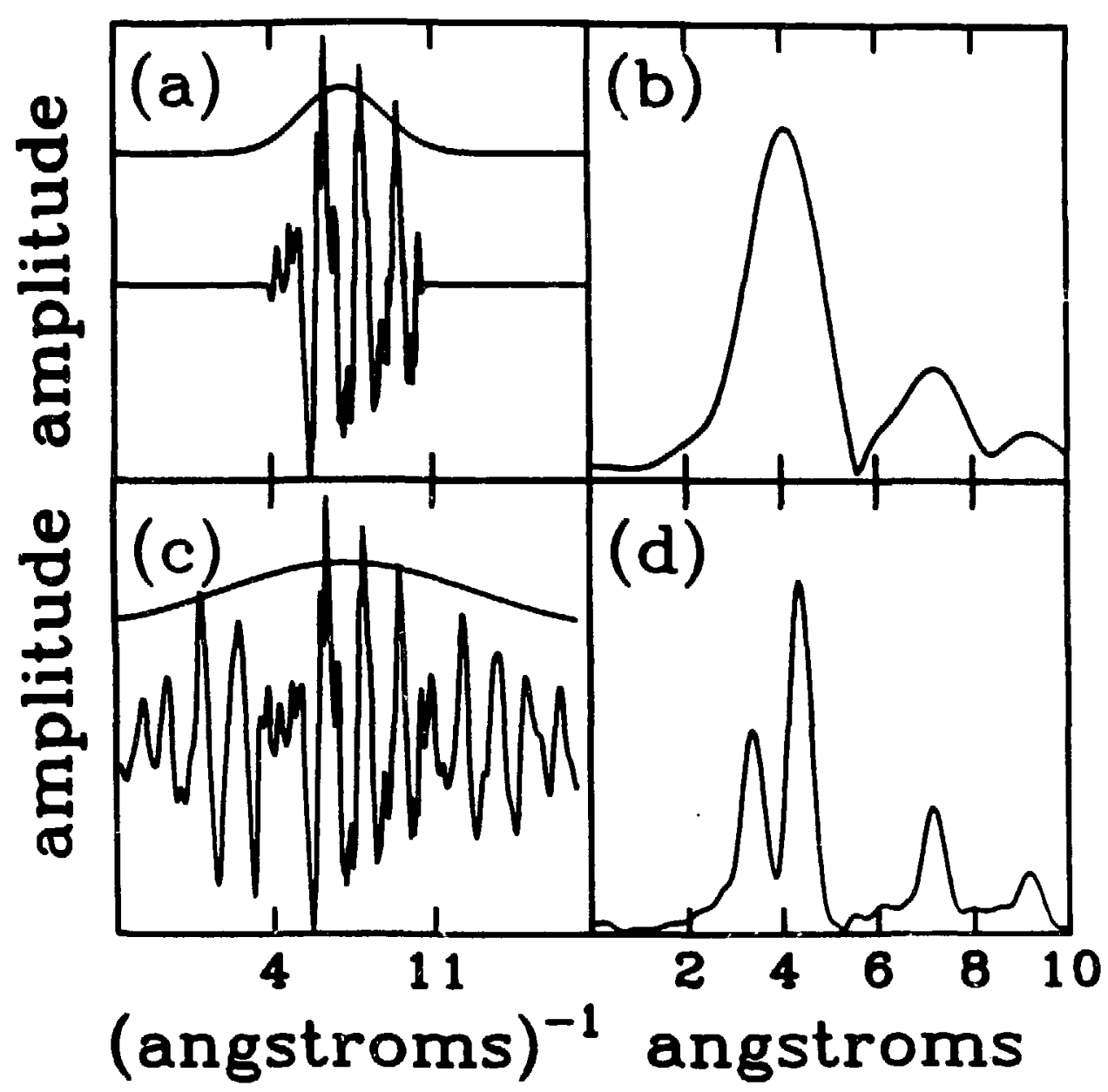

XBL $848-3545$

Figure 7 


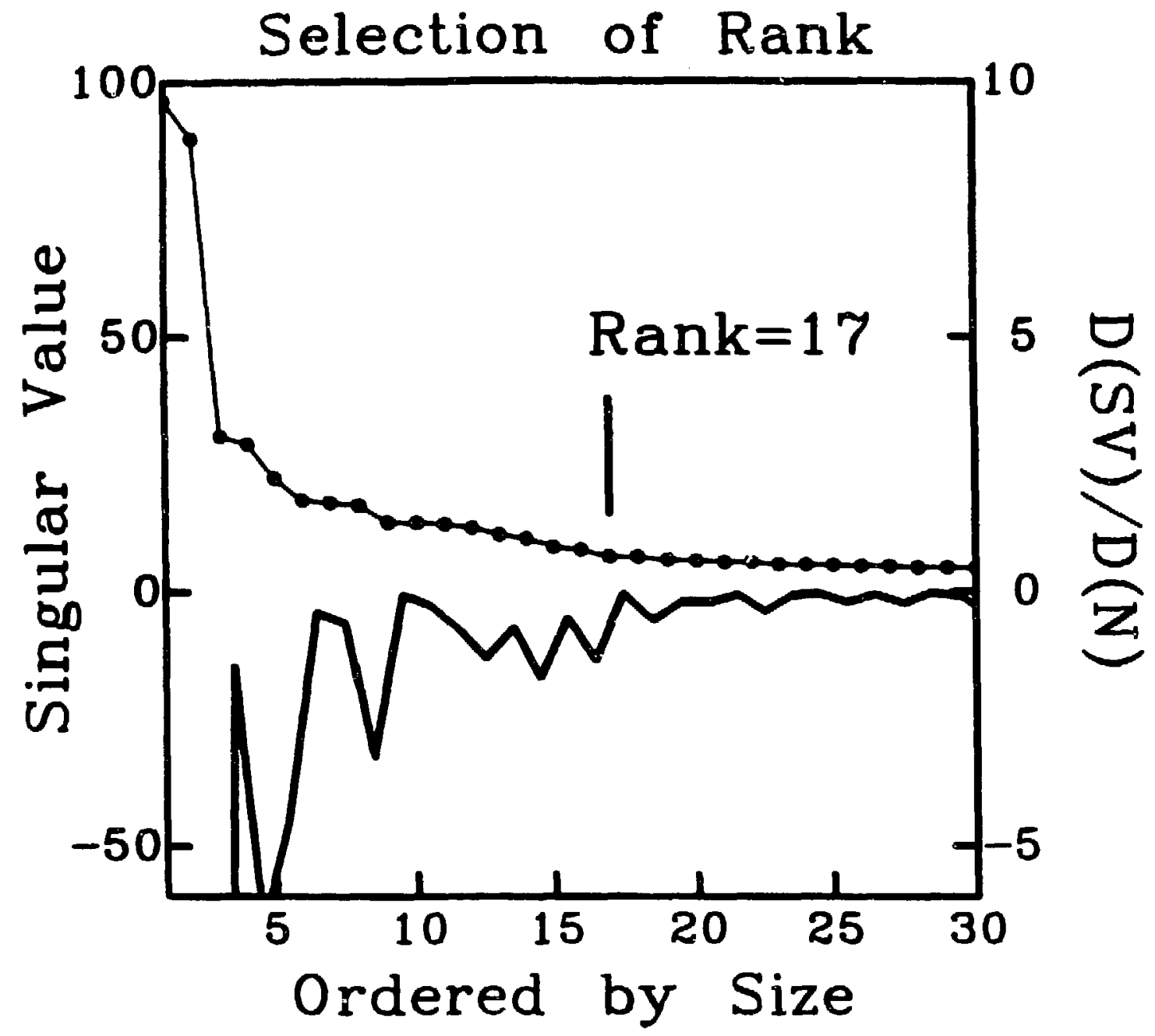

XBL 848-3549

Figure 8 


\section{CHAPTER 7:}

THE MEASUREMENT AND ANALYSIS OF ARPEFS DATA: APPLICATION TO C(2X2)S/NI(100)

ABSTRACT

We describe our procedures for deducing adsorbate geometry from core-level photoemission measurements as they are applied to $c(2 \times 2) S / N i(100)$. Extracting the oscillating part of the sulfur (1s) photoemission partial cross section gives the Angle-Resolved Photoemission Extended Fine Structure. Fourier transformation yields peaks at distances characteristic of the local site geometry and in most cases closely related to geometrical path-length differences. Multiplescattering, curved-wave calculations are fitted to Fourier filtered data to extract the geometry; the Fourier filtering reduces the size of the scattering cluster and the number of free parameters in the fit. Sources of error in this first ARPEFS measurement are discussed as a guide for future work. We find a $\mathrm{S}-\mathrm{Ni}$ bond length of $2.19 \mathrm{~A}\left(\mathrm{~d}_{\perp}=1.31\right.$ A), a S-Ni second layer bond length of 3.14 \& corresponding to a $4 \%$ expansion of the top $\mathrm{Ni}$ layer, and some evidence that $\mathrm{Ni}$ atoms in the second $\mathrm{Ni}$ layer beneath open sites in the $\mathrm{c}(2 \times 2)$ overlayer have a larger expansion from the first layer. 


\section{INTRODUCTION}

Recently, we reported ${ }^{1}$ a new approach to determining surface geometries using high-kinetic-energy, long-energy-range photoelectron diffraction measurements. Experimentally we measure a series of angleresolved core-level photoemission spectra. Thus we begin with the surface sensitivity and chemical specificity of photoemission; the angle resolution adds geometrical selectivity. As we increase the photoelectron kinetic energy, we observe intensity oscillations about an average atomic-like cross section. For electron energies from 50-500 eV, the origin of these partial cross-section oscillations-interierence between direct and scattered photoelectron waves--directly relates to the total cross-section oscillations, the extended $x$-ray absorption fine structures (EXAFS). ${ }^{2,3}$ Like EXAFS, this type of photoelectron diffraction measurement can be expressed as interference oscillations whose frequency is dominated by geometrical path-length differences and these oscillations can be frequency analyzed to display the structure information directly. To suggest these close connections and to distinguish our new approach from other techniques which use photoelectron diffraction, we will refer to the modulations in photoemission partial cross section above $50 \mathrm{eV}$ as angle-resolved photoemission extended $f$ ine structure (ARPEFS).

In this paper we discuss the analysis of $S(1 s)$ ARPEFS measured along the [011] and [001] crystallographic directions from a c(2×2) sulfur overlayer on a $\mathrm{Ni}(001)$ crystal surface. The nominal geometrical structure of this overlayer is well known. ${ }^{4-6}$ Our purpose in this paper is to report, in detail, procedures we have developed to extract the geometry of $\mathrm{S}$ on $\mathrm{Ni}$, including the distance between the $\mathrm{S}$ and the second 
Ni layer atoms from angle-resolved photoemission intensity measurements. We hope to demonstrate that these same procedures provide a basis for determining the structures of more complicated adsorbate systems. Furthermore, we discuss sources of systematic and random error which could lead to incorrect or inaccurate structures.

A simple elastic scattering theory for ARPEFS predicts that angleresolved photoemission intensity, I, oscillates about the atomic partial cross section, $I_{0}$, according to

$$
\begin{aligned}
& I=I_{0}(1+x) \\
& X(k)=\sum_{j} A_{j}(k) \cos \left[k\left(r_{j}-r_{j} \cos \theta_{j}\right)+\phi_{j}\right]
\end{aligned}
$$

where $A_{j}(k)$ contains the elastic scattering amplitude, inelastic damping, aperture integration, and thermal averaging, $r_{j}$ is the bond length, $\theta_{j}$ is the scattering angle, $\phi_{j}$ is the scattering phase function, and the sum is over all atoms $j$ with significant amplitude. Our task then is to measure $I$, the photoemission intensity, convert it to $x(k)$, and extract the path-length difference $\left(r_{j}-r_{j} \cos \theta_{j}\right)$. These three steps correspond to the three main sections of this paper. We describe the photoemission measurements in section II, the momentum scale conversion in Section III, and the frequency analysis and geometry determination in section IV. 


\section{ANGLE-RESOLVED PHOTOEMISSION}

The experimental aspects of these ARPEFS studies are discussed separately in the subsections below.

II.A. Sample

The $\mathrm{c}(2 \times 2)$ overlayer of $\mathrm{S}$ on $\mathrm{Ni}(100)$ has become one of the prototype surface structure problems both because of its importance and because of easy preparation. The bonding of sulfur to $\mathrm{Ni}$ crystals is of technological importance primarily because sulfur degrades $\mathrm{Ni}$ based catalysts. $^{7}$ The $c(2 \times 2)$ overlayer is easy to prepare either ${ }^{8}$ by segregation of bulk sulfur impurity or by decomposition of $\mathrm{H}_{2} \mathrm{~S}$ or $\left(\mathrm{S}_{2}{ }_{2}\right)$. Thus this surface system has been studied by LEED, ${ }^{4}$ surface EXAFS, 6 Normal Photoelectron Diffraction, ${ }^{5}$ and Azimuthal Photoelectron Diffraction. ${ }^{9}$ our primary concerns in selecting a system for the first ARPEFS measurements were a well known structure and a wide accessible energy range above a (1s) core level. The $c\left(2 x_{2}\right) S / N i(100)$ is ideally suited for these reasons.

The $\mathrm{Ni}$ crystal was cut on a diamond saw from a 1/4" diameter boule, oriented, and polished to $<1^{\circ}$ from the perfect (100) face. The final polish with $5 \mathrm{\mu}$ mesh $\mathrm{Al}_{2} \mathrm{O}_{3}$ powder in ethanol was followed by a 10 second etch. ${ }^{10}$ The $\mathrm{Ni}$ crystal was strapped to a resistively heated Ta sample block by $.005^{\prime \prime}$ Ta strips spot-welded to the sample block; the sample block was suspended on a 3 axis manipulator. Argon ion sputtering was effective in removing surface sulfur contamination, but each annealing cycle segregated more sulfur to the surface. Thus repeated cycles of annealing to $800^{\circ} \mathrm{C}$ and sputtering were used. When the sulfur was 
depleted, the crystal was exposed to $.5 \mathrm{~L} \mathrm{O}_{2}$ and annealed to approximately $750^{\circ} \mathrm{C}$. Experience showed that this step removes surface carbon but leaves no oxide behind. The final clean crystal was not examined by Auger or LEED prior to the $\mathrm{H}_{2} \mathrm{~S}$ exposure, to reduce the electron beam induced deposition of carbon.

The $\mathrm{Ni}$ crystal was exposed to $-2 \mathrm{~L} \mathrm{H}_{2} \mathrm{~S}$ gas from a $5 \mu$ aperture. Mass spectra of the chamber background gas during dosing showed an increase in $\mathrm{H}_{2}$ gas at the beginning of the gas exposure. Heating the $\mathrm{Ni}$ crystal to $-200^{\circ} \mathrm{C}$ produced a strong $\mathrm{c}(2 \times 2)$ overlayer pattern.

\section{II.B Electron Energy Analyzer}

The angle-resolved photoemission analyzer used for these measurements has been previously described. ${ }^{11}$ Its important features for these measurements are: 1) complete 2 axis motion allowing an unhindered selection of angles, ii) multichannel energy analysis for rapid measurements with synchrotron light, and iii) maximum $\pm 3^{\circ}$ angular resolution. The angle resolution increases for kinetic energies $\left(E_{k}\right)$ greater than the pass energy $\left(E_{p}=160 \mathrm{eV}\right)$ as the transmission of the analyzer falls like $1 /\left(E_{k}-E_{p}\right)$. The analyzer was operated for maximum transmission giving an energy resolution of $-1 \mathrm{eV}$.

\section{II.C Photon monochromator}

These $S(1 \mathrm{~s})$ photoemission measurements were made possible by the Stanford Synchrotron Radiation Laboratory's ultra-high vacuum soft $x-r a y$ double crystal monochromator. ${ }^{12}$ The Ge(111) monochromator crystal gave high flux with $1.1 \times 10^{3}$ resolving power for the $2.5 \mathrm{keV}-2.9 \mathrm{keV}$ range used for these measurements. No. detectable scattered light entered the 
sample chamber. The light is polarized in the plane of the storage ring.

\section{II.D Photoemission Measurements}

Two separate experimental geometries were used. In the first, which we call [011], the $\mathrm{Ni}(100)$ crystal was rotated about the sample normal to place a [011] axis in the plane of polarization with the [011] direction parallel to the polarization vector. The angle-resolved detector was aligned with the [011] axis; the emission and polarization vector directions were thus colinear. In the second experiment, on a different crystal, the polarization vector was pointed $30^{\circ}$ from the crystal normal in a [100] direction, and the analyzer was oriented for normal emission.

With a photon energy of $2504 \mathrm{eV}$, the electron emission spectrum was measured for 40-500 ev. This provides the electron inelastic-scattering profile and the electron energy analyzer transmission function shown in Fig. 1. For photon energies between $2535 \mathrm{eV}$ and $2894 \mathrm{eV}$, sulfur (1s) core-level photoemission measurements were made every 3 eV by advancing both the photon energy with the double crystal monochromator and the electron energy, with the energy analyzer maintaining the relation hv $=$ $E_{k}-2474 \mathrm{eV}$. A typical spectrum is shown in Fig. 1. This set of 120 photoemission spectra for each of two directions constitutes our data for the structure analysis.

\section{II.E Sources of Error}

Errors in the experimental measurements of angle-resolved corelevel photoemission limit the ultimate accuracy of our geometry 
determination. The most serious problem is the accurate angular placement of the polarization and emission direction vectors.

The polar angle of emission with respect to the crystal normal is the most important angle for geometry determination. This angle is determined by electron analyzer two axis goniometer and the sample position. The analyzer goniometer was mechanically ruled and aligned; ${ }^{11}$ it should be reproducible to $<0.2^{\circ}$, but its accuracy is limited if the sample is not placed at the center of the goniometer rotation. The sample position is determined by maximizing the photoelectron count rate; magnetic fields, incorrect alignment of the electron optics on the goniometer, and misplacement of the photon beam can lead to a sample position away from the chamber center. The sample polar angle is calibrated by laser autocollimation on the polished face of the crystal. The optical surface can be $\pm 1 / 2^{\circ}$ from the ideal (100) face; subsequent in vacuo cleaning could increase this difference. The laser autocollimation is referred to the surface of a vacuum chamber window; the angle between the window and the analyzer goniometer must be inferred from the construction of the window and the vacuum chamber. Once calibrated, the sample position is determined by rotation of a sample manipulator; errors may be introduced if the crystal does not lie on the axis of rotation or if liquid nitrogen cooling coils or electrical wires apply torque to the sample while it is reoriented. Combined, these errors may well be as large as $\pm 2^{\circ}$, although some geometries, e.g. normal incidence light, normal emission, or sample normal oriented perpendicular to the autocollimation reference window, can be double-checked more easily. 
The azimuth of emission and polarization is determined by visual alignment of Low Energy Electron Diffraction spots with respect to the vacuum chamber base; although fairly crude, this procedure can be rather precise. The LEED spots report the surface crystallography directly and only a strong magnetic field across the LEED apparatus axis would affect the azimuthal position of these spots; spots separated by $-10 \mathrm{~cm}$ can be aligned to within $\pm 1 \mathrm{~mm}$ to give a $1^{\circ}$ error.

The polarization vector position is less important for surface geometry. The synchrotron light is polarized in the horizontal plane. Since the position of our sample is referred to our vacuum chamber, we place the vacuum chamber along the photon beam by centering the beam at the entrance to the chamber and at the electron analyzer on phosphorescent screens. The rotation of the chamber about the beam is then set with a mechanical level. It is difficult to estimate the final error in alignment, but the most sensitive angle--the rotation about the beam--can be reproduced to $<1^{\circ}$.

Other errors in the photoemission measurements primarily affect the measured ARPEFS amplitude and not the oscillation frequency. Steps on the crystal surface or an improper dose of $S$ atoms will lead to $S$ photoemission not representative of an ordered overlayer; this will typically reduce the measured oscillations as they are expressed as a fraction of the total partial cross section.

Although this catalog of errors is a rather dreary list, it is roughly the state-of-the-art in angle-resolved photoemission measurements with synchrotron radiation. In reviewing this list one should recall that the apparatus used for this first ARPEES measurements was not designed for high precision structure determination. The more 
serious problems detailed above can be remedied easily now that their importance is understood. 


\section{THE EXTENDED FINE STRUCTURE}

\section{III.A Method}

From the raw photoemission measurements we must derive the oscillations in the partial cross section. We have developed a threesicep procedure which relies only on photoemission measurements. These steps are:

i) estimation of the photoemission intensity at each kinetic energy by non-linear least-squares fits to a simple line shape function,

ii) normalization of these intensities for photon flux and electron analyzer transmission variations using background intensity measurements, and

iii) estimation and removal of the atomic partial cross section, $I_{0}$

In this section we examine each of these steps in detail. Before proceeding, we pause to discuss the energy scales involved in the analysis. Our raw data consists of a series of photoemission spectra for increasing photon energy, hv. Each spectrum is centered on the sulfur is core-level photopeak but includes $-10 \mathrm{eV}$ of the electron emission spectrum to higher and to lower kinetic energy. The photopeak mean kinetic energy, $E$, is related by the $S(1 s)$ binding energy, $E_{B}$, to the photon energy, hu:

$$
E=h v-E_{B}
$$

Our measurements and our analysis depend only on the photopeak kinetic energy, E; we do not use the monochromator energy scale or the value of 
the binding energy. We label each spectrum by the photopeak mean energy, $E$, and electron energy within each spectrum we will call $E_{a}$. To estimate the photoemission intensity, we decompose each photoemission spectrum into peak, tail, and background contributions. Fig. 1 demonstrates the decomposition for $E=264 \mathrm{eV}$. Notice that the least-squares fit also provides the value of $E$. We employ simple functions for our fits. The Gaussian function for the photopeak, centered at $\mathrm{E}$,

$$
G\left(E, E_{a}\right)=G(E) e^{-\left(E a^{-E}\right)^{2} / 4 \sigma^{2}}
$$

has an area $G(E)$ and a width $\sigma$. The tail is a Gaussian broadened step function:

$$
T\left(E, E_{a}\right)=T(E)\left[\frac{1}{2}-\frac{1}{2} \operatorname{erf}\left(\frac{E_{a}-E}{2 \sigma}\right)\right]
$$

whose mean is at $E$ and whose width is forced to be the same as the photopeak. The background is a scaled experimental electron emission spectrum, $M$, taken with $E=30 \mathrm{eV}$ :

$$
B\left(E, E_{a}\right)=B(E) * M\left(E=30, E_{a}\right)
$$

From each least-squares fit we derive three numbers, the Gaussian area, $G(E)$, the photopeak position, $E$, and--for reasons we now discuss--the background scale factor $B(E)$. 
The Gaussian areas derived from our least-squares fits are proportional to the partial cross section we seek, but they also depend on the photon flux and electron analyzer transmission function. Calling the partial cross section $I(E)$, the photon $f l u x F(E)$, and the transmission function $A(E)$, we have the Gaussian areas

$$
G(E)=F(E) * A(E) * I(E)
$$

Note that the photon flux, $F(E)$, is written as a function of photoelectron energy, E. We mean for this function to represent all the instrumental intensity variations which influence the strength of the photoemission spectrum measured at kinetic energy, E. Thus F(E) contains the photon monochromator transmission, storage ring current, slit widths, effective sample-photon-analyzer interaction region, and the spectrum integration time.

To remove the "photon flux" contribution we model the photoemission background as the product of photon flux, analyzer transmission, and an intrinsic background function, $N\left(E, E_{a}\right)$ :

$$
M\left(E, E_{a}\right)=F(E) * A\left(E_{a}\right) * N\left(E, E_{a}\right)
$$

We then assume that the intrinsic background does not depend on photon energy from 50 to $500 \mathrm{eV}$ above the absorption edge:

$$
M\left(E, E_{a}\right)=F(E) * A\left(E_{a}\right) * N\left(E_{a}\right)
$$

Thus a measurement of the electron emission spectrum for $E_{a}=$ 50-500 eV--when the photopeak is at $E=30 \mathrm{eV}$--is proportional to the intrinsic background: 


$$
M\left(E=30 \mathrm{eV}, E_{a}\right)=F(30) * A(E) * N\left(E_{a}\right)
$$

When this spectrum is used as the photoemission background in the Ieastsquares fits, a scale factor $B(E)$ is introducec (eqn. 1). Since we assume that the intrinsic background, $N\left(E_{a}\right)$, does not depend on the position of the photopeak, the scale factor must be the ratio of the flux and transmission during the ARPEFS measurement to the flux and transmission during the background scan:

$$
B(E)=\frac{F(E) * A\left(E_{a}\right)}{F(30) * A\left(E_{a}\right)}=F(E) / F(30)
$$

Therefore the ratio of the photopeak area to the scale factor for the background function is proportional to the product of partial cross section and analyzer transmission:

$$
G(E) / B(E)=I(E) * A(E) / F(30)
$$

Only the analyzer function remains. Based on the discussion in Section II, we take $A(E)=1 / E$, to give the partial cross section as:

$$
I(E)=c G(E) * E / B(E)
$$

with $c$ an arbitrary, unknown constant.

Fig. 2 demonstrates the $I(E)$ curve we obtained from applying these ideas to the $S(1 \mathrm{~s})$ ARPEFS data. Notice that while our description of our processing is complicated, the actuel analysis is quite simple. The least-squares fits have three Inear parameters (Gaussian area, tail area, and background scale) and two non-linear parameters (Gaussian width and mean). Our least-squares fit computer program records these parameters on disk; when all the photoemission data have been analyzed, 
the parameters are read back in and the array algebra of equation (2) is performed.

Now we extract the oscillating part of the partial cross section according to

$$
x(E)=\left(I-I_{0}\right) / I_{0}
$$

In principle the atomic partial cross section, $I_{0}$, could be approximated by the sulfur cross section calculated from some wavefunction for free atomic sulfur or a model for the $\mathrm{S}$ on $\mathrm{N} I$ problem. From free atom crosssection calculations we can see that the sulfur $I_{0}$ contains only very low frequency information: We will make little error at the strlicturally important frequencies if we approximate $I_{0}$ as the smooth part of I. Furthermore, as we discuss below, systematic errors in the measurement of I and the estimation of low frequencies in the Fourier transform invalidate any distinctions between the "correct" $I_{0}$ and our simple estimate.

Eig. 2 shows our fit of $I(E)$ to a quadratic function of energy:

$$
I(E)=a E^{2}+b E+c
$$

for the [011] experiment and a smooth spline for the [001] experiment. The resulting $x(E)$ from eqn. (3) is shown in Fig. 3 .

This curve, $\chi(E)$, is the Angle-Resolved Photoemission Extended Fine Structure. It represents the proportional shange in partial cross section due to interference between direct and scattered photoemission. Our measured oscillations are very large, $\pm 50 \%$ of the average value; 
the signal seems to be dominated by a few mid-range frequencies. In the next section we demonstrate that these oscillations can be analyzed to determine surface structures.

\section{III.B Sources of Error}

The procedure we selected to derive the extended fine structure from the photoemission measurements suffers from several systematic and random errors. Deriving the partial cross-section curve $I(E)$ from the photoemission measurements may suffer from five major problems:

i) cross-section variations. As the photon energy is scanned, the inelastic electron spectrum, $N(E)$, may change as the photoabsorption cross section for the levels which contribute to it change. Far from threshold these changes will be smooth decreases in electron flux. Crossing a threshold will cause a sudden jump in flux. For $\mathrm{S}$ on $\mathrm{Ni}$ there are no absorption thresholds, and since we measured $N(E)$ at a photon energy near threshold where the cross-section is large, we expect that our background will be systematically too high at higher photon energies.

ii) Auger peak cross-section variation. The inelastic electron spectrum measured with the photopeak at low kinetic energy should overestimate the size of the adsorbate Auger features. For example, the S LMM Auger region near $150 \mathrm{eV}$ was measured with a photon energy of $2504 \mathrm{eV}$, but was then used to fit photoemission spectra with photon energies near $2624 \mathrm{eV}$. The drop in the absorption cross section over this energy range will lead to a smaller least-squares fit coefficient, $B(E)$, for the background in the Auger region than we would have obtained 
if we had measured the higher region with a photon energy closer to 2640 eV. Thus we will overestimate $I(E)$ near adsorbate Auger features.

iii) Storage ring current loss. The inelastic scattering curve was estimated by a single long energy range scan of the background. The storage ring current will drop by $-5 \%$ during this scan, leading to a slight underestimation of $M\left(E, E_{a}\right)$.

iv) Photon beam movement. One further problem with the background fit method stems from the use of the double crystal JUMBO monochromator at the Stanford Synchrotron Radiation Laboratory (SSRL). Heat from the synchrotron beam on the first crystal expands it, changing the spectrometer equations of motion. While a static heat load can be compensated, synchrotron beam decay, beam loss or re-injection changes the heat load. While the $-3 \mathrm{eV}$ energy shifts which accompany a doubling of beam current on injection do not affect our spectroscopy--we measure the kinetic energy--the beam movement on the sample can change the shape of the background. .

v) Photopeak lineshape function. The choice of Gaussian photo-peak plus Gaussian tail to represent the photoemission intensity is certainly oversimplified. Although the instrumental resolution is $-2.5 \mathrm{eV}$, our measured photopeak had a width of $-3.0 \mathrm{eV}$, indicating additional broadening. The tail contains electrons scattered inelastically in the sample and in the analyzer as well as the metallic response tail of Sunjic and Doniach. The processes would have to be investigated as functions of photoelectron kinetic energy to accurately characterize the photoemission lineshape. Our much simpler function slightly underestimates the true line shape, giving a low value for the photoemission intensity. 
The conversion of photoelectron intensitles to fine structure leads to two further systematic errors. First, the electron analyzer transmission is only approximately proportional to $1 /$ (electron kinetic energy): Second, our method of determining $I_{0}$ empirically from our measurements will mix the true atomic partial cross section with very low frequency-interference oscillations and systematic errors of the types we have been fiscussing.

None of these systematic errors is expected to contribute to the mid-range frequencles important for structure analysis. Except for the Auger intensity problem, these errors should lead to mild trends in the idata which will be removed in the calculation of $x(k)$. The Auger problem will be concentrated at the kinetic energies of the Auger peak: The Fourier spectrum of this disturbance will be broad and not peak at structure frequencies.

Our random errors come from the statistical accuracy of our photoemisgion measurement. Assuming no errors in the lineshapes of the least-squares fits and assuming a normal distribution of noise, the standard error of the partial cross section, $\sigma_{I}$, divided by the partial cross section, I, i.e. the inverse of the signal-to-noise ratio, will be given by

$$
\left(\frac{\sigma_{I}}{I}\right)^{2}=\left(\frac{\sigma_{a}}{G}\right)^{2}+\left(\frac{\sigma_{E}}{E}\right)^{2}+\left(\frac{\sigma_{B}}{B}\right)^{2}
$$

where $\sigma_{j}, j=C, E, B$ are the standard errors of each parameter in the formula for I. Each standard error will be proportional to the residual variance which-given our assumptions--will be proportional to the total number of counts in the spectrum. Numerical calculations of the 
standard errors show that the random errors will contribute an approximately flat background to the Fourier spectrum of our signal. For very low signal-to-noise power ratios, spurious peaks in this background could be misinterpreted or contribute erroneously to correct scattering peaks. Our spectrum has sufficient precision to avoid this problem. 


\section{EXTRACTING GEOMETRY INFORMATION}

In the previous section the extended fine structure, $\chi(E)$, was derived from a series of angle-resolved photoemission measurements. In this section we analyze the fine structure to extract the geometry. We divide the entire procedure in two parts, Fourier analysis and multiple scattering analysis. We will discuss errors after we have described the entire procedure.

\section{IV.A Fourier Transformation}

There are three steps in the Fourier transform procedure:

conversion from energy to momentum scales, tapering or autoregressive linear prediction, and Fourier transformation.

Conversion of the fine structure curve from a kinetic energy scale to a momentum scale uses the de Broglie relation,

$$
E=\frac{k^{2} k^{2}}{2 m}
$$

to relate the electron's energy to its wave vector magnitude. For the electron energy we use the peak position, $E$, derived from the leastsquares fit to eliminate any energy errors in the photon monochromator. As discussed in ref 13 the measured electron energy can be related to the energy of the electron during the scattering $E_{S}$ by $E=E_{O}+E_{S}$, where $E_{0}$ the solid's inner potential. Thus the wavenumber, $k$, for a kinetic energy $E_{k}$ is given by

$$
k=\frac{2 m}{n^{2}}\left(E-E_{0}\right)^{1 / 2} \text {. }
$$


With this conversion we obtain $x(k)$ as a table of numbers $(k, x(k))$ whose spacing in $k$ depends on the energy spacing of the photoemission measurements and on $E_{0}$. Unfortunately none of the subsequent analysis can process data with unequal increments in the abscissia. Therefore we fit $x(k)$ locally to a numerical spline function and evaluate the spline on an equally spaced mesh of 128 points. Fig. 4 shows the interpolated $x(k)$ for $E_{0}=10.5 \mathrm{eV}$.

The second step in frequency estimation is required to reconcile the concept of frequency analysis with the finite range of our experimental measurements. Our goal is the isolation of the path-length difference, $r_{j}-r_{j} \cos \theta_{j}$, from the experimental $x(k)$ which we believe is represented by a cosine series, eqn. (3), suggesting a Fourier analysis procedure. As discussed in ref. 14, direct Fourier series transformation of $x(k)$ would not be adequate: Fourier analysis assumes an infinitely long measurement range. Finite range data must be tapered smoothly to zero by a weighting function before Fourier analysis, or else some procedure such as the autoregressive linear prediction described in ref 14 must be applied to estimate the frequencies of oscillation from a finite measurement range. The Fourier spectrum of the weighted data will be a smoothed version of the spectrum of scattering amplitude versus path-length difference while the autoregressive linear prediction Fourier transform has higher resolution but is more sensitive to $k$ dependence in the envelope which multiplies individual cosine oscillations. Therefore we will present results from both procedures, using the ARLP spectra as a qualitative guide to the frequency spectrum. Since we will ultimately refine the geometry by direct comparison to the experimental oscillations, the choice between 
conventional and ARLP methods to solve the finite data range problem in the Fourier transform is not important.

Following the guidelines in ref 14 we multiply the interpolated $\chi(k)$ curve by a Gaussian centered at $7.5 \mathrm{~A}^{-1}$ and having a full width at half maximum of $4 \mathrm{~A}^{-1}$ for the conventional taper weighting method. For the ARLP method, 64 autoregressive coefficients were fit to the data based on 14 singular values for the [001] experiment and 17 singular values for the [011] experiment; the ARLP was applied to extrapolate 128 points forward and backward, and the resulting oscillations were tapered with a Gaussian function centered at $7.5 \mathrm{~A}^{-1}$ and having a full width at half maximum of $12.3 \mathrm{~A}^{-1}$. This prepares the oscillations for Fourier transformation.

In the third and final step we apply the Fourier transform via the Fast Fourier Transform algorithm. 15 prior to transformation we add zeroes to give 2048 points; this increase interpolates the Fourier spectrum to give smooth peaks. 14

The magnitude of the complex Fourier coefficients is displayed in Fig. 5 for the [011] experiment, and Fig. 6 for the [001] experiment. Since our unit for $k$ is $\mathrm{rad}^{-1} \mathrm{~h}^{-1}$, the independent axis of our Fourier transform gives the path-length difference directly in A. Each of the peaks in Figs. 5 and 6 represent one or more scattering interferences. The peak position will be near the geometrical path-length difference, $r_{j}-r_{j} \cos \theta_{j}$, plus the linear part of the scattering phase shift $\phi_{j}$. When comparing our results to plots of surface EXAFS Fourier transforms, recall that the EXAFS scale is usually chosen to display the bond length, $r_{j}$ : the scattering path length is just twice as long. 
The assignment of the peaks in the [011] transform, Fig. 5, to particular scattering path lengths has been the subject of some controversy. ${ }^{16}$ We will repeat our previous, simple-minded assignment based on the empirical observation that nearest neighbors and backscattering atoms dominate the spectrum; ${ }^{1}$ there is as yet no theoretical calculation which reproduces the Fourier peaks with enough accuracy to contradict this idea. However, as noted below, a peak at $2 \mathrm{~A}$ due to a side-scattering nearest neighbor atom does not appear reliably in the Fourier transform spectrum.

Two peaks in the spectrum are primarily due to three $\mathrm{Ni}$ nearest neighbors. The largest peak--at $4.4 \mathrm{~A}$--corresponds to scattering from the nearest neighbor $\mathrm{Ni}$ directly behind the sulfur atom from the detector. With a bond length of $r_{j}=2.2 \mathrm{~A}$ and a scattering angle $\theta_{j}=$ $171^{\circ}$, the path-length difference is $4.37 \mathrm{~A}$. All of the amplitude factors favor this scattering atom. It lies along the peak in the photoemission final state; it lies close to the emission center; and it backscatters into the detector.

The second largest peak--at 3.2 A--corresponds to electron scattering from two nearest-neighbor $\mathrm{Ni}$ atoms. These atoms are symmetrlcally located on either side of the plane containing the surface normal (the [001] direction) and the emission vector (the [011] direction). The scattering angle is $116^{\circ}$, giving a path-length difference of 3.12 A. Despite the combined scattering power of two atoms, this peak is smaller than the 4.4 A peak: both the photoemission final state angular distribution and the scattering angular distribution are less favorable for $116^{\circ}$ scattering. 
The fourth nearest-neighbor atom should have a scattering angle of $83^{\circ}$ and thus would appear at a path-length difference of $1.96 \mathrm{~A}$. However we expect 1 ts amplitude to be small because the atom is near the photoemission final state node: $\cos 83^{\circ}=0.12$. This atom does not produce a rellable peak in the Fourier transform. For example, it is small in Fig 5, while it was somewhat larger in earlier analyses ${ }^{1}$ us: ng a slightly different transform weighting.

The peaks at $7.5 \mathrm{~A}$ and $9.5 \mathrm{~A}$ seem to correspond to backscattering atoms further away from $S$ along the [011] axis. If we consider the 4.4 A peak to be a member of a (011) plane perpendicular to the emission direction, then the 7.5 \& peak would correspond to 4 atoms in the next (011) plane away from $S$ and the 9.5 A peak would correspond to the single atom in the succeeding plane which lies directly behind the $4.4 \mathrm{~A}$ Ni scatterer.

We should note that the latest single-scattering cluster calculations ${ }^{16}$ with improved treatment of thermal averaging agree with our assignment of the $4.4,7.5$ and 9.5 \& peaks, leaving the 3.2 \& peak as unsettled. The basic picture in terms of path-length differences is also not affected by multiple scattering since, in the intermediate 'nergy range, forward focusing is the dominant effect of multiple soattering. ${ }^{17}$ In summary, we believe that the overwhelming weight of evidence favors the simple interpretation given earlier ${ }^{1}$ : that the Fourler transform peaks arise primarily from a few identifiable atoms. of course allowance must be made for possible interference due to nearlying path length differences and Ramsauer-Townsend splitting. However, this does not negate our basic conclusion, based on strong experimental evidence, that a small number of path lengths are actually present with 
any appreciable intensity in the data. The fallure of oversimplified theories to reproduce the data only confirms the shortcomings of those theories.

Two peaks in the normal emission [001] experiment Fourier spectrum can be assigned in the same fashion as the [011] assignment. The $6.2 \mathrm{~A}$ peak must be predominately backscattering from the second layer $\mathrm{Ni}$ atom directly below $S$ since this atom is $180^{\circ}$ from the detector in the [001] emission geometry. The peak near 10 A should have a large contribution from atoms in the third $\mathrm{Ni}$ layer below $\mathrm{S}$ for the same reason. Atoms in the second $\mathrm{Ni}$ layer not directly in backscattering contribute the majority of the signal to the Fourier spectrum between 6.2 and $9 \mathrm{~A}$, but we cannot give more specific assignments without detailed calculation.

The most interesting features of the [001] Fourier transform are the two peaks below $5 \mathrm{~A}$ which seem to defy a scattering path-length explanation. Both of these peaks can be attributed to scattering from the four nearest-neighbor $\mathrm{Ni}$ atoms in the first $\mathrm{Ni}$ layer below $\mathrm{S}$ even though the geometrical path-length difference for all four neighbors is near 3.5 \& where no Fourier peak is observed. The physical explanation for this Fourier peak splitting is a generalized Ramsauer-Townsend resonance in the $\mathrm{Ni}$ scattering amplitude which simulates a beat envelope as $k$ increases through $7.5 \AA^{-1}$. We have discussed these peaks and their use in the measurement of the S-Ni bond length alsewhere. 18

\section{IV.B Multiple Scattering Analysis}

With the raw photoemission spectra reduced to ARPEFS oscillations and the Fourjiz spectra at hand we can proceed to determine the structure. Previously, we have attempted to analyze the $4.4 \mathrm{~A}$ 
backscattering peak in the [011] experiment by applying the Fourier backtransformation methods of EXAFS. ${ }^{1}$ This analysis was based on the apparent success of single-scattering calculations to simulate the general features of the ARPEFS curve, but we now recognize ${ }^{13}$ that forward focusing is a fundamental feature of the photoelectron scattering. While the forward focusing does not change the oscillation frequency, it does change the oscillation amplitude and phase. Since the EXAFS-like analysis requires the phase to be known, ${ }^{3}$ we will not pursue that approach here.

Our alternative is a Fourler-filtering, least-squares fitting procedure which uses the Fourier spectrum to reduce the multiple parameter space of geometry variables without relying on the Fourier transform for the final structure analysis. The key element in this approach is the filtering of the ARPEFS to remove scattering path-length differences corresponding to all layers except the $S$ overlayer and the first Ni layer. This filtered ARPEFS curve then depends upon a single geometrical parameter, the $\mathrm{S}-\mathrm{N} \mathrm{i}$ bond length, or equivalently the $\mathrm{S}-\mathrm{N} \mathrm{i}$ interlayer spacing $\left(d_{1}\right)$. Furthermore, the filtered curve contains only a restricted set of path-length differences and numerical simulation of the filtered curve even including multiple-scattering, and curved-wave corrections is very economical. Once the $\mathrm{S}-\mathrm{Ni}$ layer spacing is set, the spacing to the second layer can be optimized by selecting a new filter width which includes atoms scattering from the second layer.

For the [001] experiment, it is appropriate to filter the Fourier spectrum at $5 \mathrm{~A}$, isolating the two peaks split by the $\mathrm{Ni}$ scattering resonance. As this analysis involves a discussion of the resonance, we 
have reported it separately ${ }^{18}$ finding a S-Ni bond length of $2.20 \pm .02 \mathrm{~A}$ $\begin{aligned}\left(d_{1}\right. & =1.32 \pm .03 \mathrm{~A}) . \\ & \text { For the }[011] \text { experiment, } 5 \text { A was also chosen for the filter }\end{aligned}$ cutoff. This location is a minimum in the Fourier amplitude spectrum just above the $4.4 \mathrm{~A}$ main backscattering peak. To obtain the filtered ARPEFS spectrum we have simply zeroed the Fourier coefficients for frequencies above $5 \mathrm{~A}$ and applied the Fast Fourier inverse transform. We recognize that the [011] experimental geometry is more difficult to align with our present apparatus than the normal emission [001] case, and we have noticed that our numerical simulations are very sensitive to the polar angle of emission. Thus we have performed a two dimensional search in $\mathrm{S}-\mathrm{Ni}$ interlayer spacing and emission polar angle to minimize the possibility that misalignment determines our result. Fig. 7 gives the least-squares error surface for these variables. The numerical simulations were performed as described in ref. 13 using the non-structural parameters given there. Moderate changes in the nonstructural parameters will change the size of the least-squares error but not the position of the minimum; conversely, we cannot reliably estimate the non-structural parameters by least-squares fits of this kind. A clear minimum is evident in the surface at $43^{\circ}$ emission angle and a S-Ni interlayer spacing $\left(d_{L}\right)$ slightly above $1.30 \AA$, in good agreement with the results of the [001] experiment.

With the emission angle for the [011] experiment fixed at $43^{\circ}$ and the $\mathrm{S}-\mathrm{Ni}$ interlayer spacing $\mathrm{fixed}$ at $1.30 \mathrm{~A}$, we can return to the Fourier spectrum and filter for the second $\mathrm{NI}$ layer. An appropriate filter location for the [011] experiment is $10.5 \mathrm{~A}$, but the [001] spectrum should be cut somewhat lower to avoid path lengtins near 10 \& 
due to scattering from third layer $\mathrm{Ni}$ atoms. However we have used 10.5 \& for both experiments for convenience in the numerical simulation. The interlayer spacing between the second and third $\mathrm{Ni}$ layers is anyway equal to the bulk interlayer spacing ( 1.76 A) to within our ability to measure it at this time.

In refining our geometry we must recognize that the $c(2 \times 2)$ symmetry observed in LEED does not constrain the $\mathrm{Ni}$ atoms in the second layer to be coplanar. Half the $\mathrm{Ni}$ atoms in this layer lie directly below $\mathrm{S}$ atoms (we cali these atopped atoms) and half of these atoms lie below open spaces in the half monolayer coverage (we call these open atoms). The stability of the $c(2 \times 2)$ overlayer suggests that the local electronic environment of atopped and open $\mathrm{Ni}$ atoms could be different leading to the possibility that they would seek different equilibrium distances from the first $\mathrm{N} i$ layer. Therefore we have refined the positions of the atopped and open atoms separately, giving, for the [001] geometry, the two dimensional least-squares error surface in Fig. 8. The dashed line running diagonally indicates the cut through this surface on which atopped and open atoms are coplanar. Along this line a clear minimum is found near 1.82 f for the first and second $\mathrm{Ni}$ layer spacing. To be more precise our measurement gives the distance between $\mathrm{S}$ and the second $\mathrm{Ni}$ layer of 3.12 \& which we combine with the spacing of the $S$ and first $N i$ layers to give 1.82 \& for the $\mathrm{Ni}-\mathrm{Ni}$ spacing. Relaxing the coplanar constraint, we see a broad minimum where the atopped $\mathrm{Ni}$ atoms stay near 1.82 \& below the first layer while the open $\mathrm{Ni}$ atoms move further down with a minimum near 1.87 A.

The surface for the [011] experiment is simllar along the coplanar constraint line having a minimum near $1.84 \mathrm{~A}$, but once the open atoms are 
allowed to vary independently, no minimum is found for spacings less than $1.94 \mathrm{~A}$. He discount the significance of this result because the [011] experimental geometry is not sensitive to the frequency change which accompanies the displacement of the open atoms-they move away at an oblique angle--but it is very sensitive to the amplitude of the scattering from these atoms. In fact, of all the scattering events which contribute to the two ARPEFS curves discussed here, calculations of scattering from open atoms in the [011] experiment have the poorest agreement with experiment.

These comparisons of scattering calculations and Fourier filtered experimental data rely on an accurate value for the inner potential used to construct the experimental momentum scale. We can esiimate the maximum possible geometry error by calculating the least-squares error after optimizing the fit between experiment and theory with an adjustable inner potential. Since most of the ARPEFS signal is already contained in the 10.0 A simulations, we recalculated the least-square error surface for the [001] experiment comparing these simulations directly to the experimental oscillations on the experimental energy scale, allowing both the theoretical inner potential and overall scale factor to vary. The resulting surface is shown in Fig. 10. Since the minimum in the surface with fixed inner potential does not improve when the inner potential is varied, the minimum shifts, and, with the added flexibility of the scaling and shifting optimization, the minimum will be much broader. We find a broad minimum centered near 1.85 A for atopped $\mathrm{Ni}$ atoms and $1.87 \AA$ for open $\mathrm{Ni}$ atoms, a slightly greater expansion than that found with fixed inner potential. 
The optimal inner potential varies monotonically with the spacing between the sulfur layer and the atopped $\mathrm{N} 1$ layer; when this spacing is, for example 1.86 , the optimal inner potential is $-7.8 \mathrm{eV}$ and larger expansions give lower inner potentials. Thus as long as we believe that the inner potential should be near $10 \mathrm{eV}$, the error surface with variable inner potential represents our maximum error: any restraint on the inner potential to bring it back toward $10 \mathrm{eV}$ will bring the optimal geometry back toward $1.87 \mathrm{~A}$. We have also varied the inner potential in the analysis of the ARPEFS curves filtered at 5 \& by placing the filtered experimental data on an energy scale using the inverse of the original conversion of energy to momentum. Both the [001] and [011] experiments give unchanged optimal $\mathrm{S}-\mathrm{N} 1$ spacing and optimal inner potentials between 10 and $11 \mathrm{eV}$. Thus we believe the variable inner potential surface result represents an upper bound to the interplanar spacing of $1.86 \mathrm{~A}$.

Finally, we have selected a fixed spacing of the sulfur and second $\mathrm{Ni}$ layers at $3.135 \mathrm{~A}$ with atopped and open atoms coplanar and reoptimized the $\mathrm{S}-\mathrm{Ni}$ interlayer spacing calculating all path lengths up to $10.5 \mathrm{~A}$ and using the same two error criteria as discussed above. The error curves in Fig. 11 all have their minima slightly above 1.30 A (d $)$ (2.19 A S-N1 bond length) with the [011] curve to the spacing being more sensitive.

We have based our quantitative analysis on the least-squares error criterion, but visual comparison of the curves confirms the conclusions of the numerical analysis. We can verify that $d_{\perp}=1.30 \mathrm{~A}$ fits the filtered ARPEFS better than $d_{\perp}=1.35$ A as in Fig. 12; by comparing 
Figs. 13 and 14 we can certainly exclude a $S$ to second layer spacing of $3.06 \AA$ in favor of one closer to $3.14 \mathrm{~A}$.

IV.B Sources of Error

The paramount sources of error in our structure analysis are the value of the inner potential and the scattering phase shifts used in the multiple-scattering calculations. Substantially less important are the values chosen for the non-structural parameters in the theory, which control the oscillation amplitude but not its phase or frequency.

We strongly emphasize that the precision of ARPEFS analysis relies on the energy width of the measurement. Over the course of a single oscillation, a constant phase error, due to inner potential or scattering phase shifts, will lead to significant apparent geometry changes. Only by comparing the oscillations over several cycles can this source of error be reduced. Furthermore, estimation of the atomiclike background, $I_{0}$, severely distorts oscillations with a single cycle over the energy range, and the Fourier processing requires a maximum energy range for resolution of the Fourier peaks. Whenever several ARPEFS oscillations are covered in the measured range, the precision of the structure analysis should exceed 0.02 A in interplanar spacings.

We cannot be so sure about the accuracy. As we have discussed in the previous section, the inner potential is directly connected to the structure determination, and we find that a $2 \mathrm{eV}$ error leads to a 0.02 A error in geometry. The errors caused by the scattering phase shifts are more difficult to assess particularly since there does not seem to be published phase shifts in this energy range to which we may compare. As we are primarily sensitive to backscattering and forward scattering, we 
can conclude that the Irequency shift caused by errors in the scattering phase shifts are likely to be negligible: we can see from published ${ }^{19}$ EXAFS backscattering phase functions that change from $\mathrm{Ni}$ to $\mathrm{Cu}$ potentials would introduce a linear phase shift less than $0.02 \mathrm{~A}$, and since the linear part of the forward scattering phase function is less than 0.05 A even a $50 \%$ error may be 1gnored. Exactly the opposite must be concluded about errors due to the constant part of these phase functions: The published backscattering phase functions have large changes in phase with atomic number, and the forward scattering constant phase shift is large.

There is moreover there is a close connection between inner potential errors and errors in the constant part of the scattering phase function. This connection is exploited in the analysis of EXAFS data 20 by allowing the inner potential to vary. The procedure we followed in the previous section to vary the inner potential is analogous to the EXAFS analysis in that we might hope to cancel some errors in the constant phase with a variable inner potential, but we note several differences. First, the EXAFS inner potential is a complex weighted sum of absorption edge energies even when the scattering potential is exactly known: for all practical purposes the EXAFS inner potential is not calculable or measurable. The ARPEFS inner potential nay be more accessible if only because it is not connected to the photoabsorption process. Second, the EXAFS inner potential usually must also account for errors in phase shift functions caused by curved wave corrections, while our simulation curves include curved wave effects. And third, the EXAFS analysis usually concentrates on a single backscattering oscillation so that the floating inner potential need not work to 
correct amplitude errors while our floating inner potential may compromise between correcting phase errors and errors due to incorrect relative scattering amplitudes. Thus the simpler analysis of the $5 \mathrm{~A}$ filtered data lead to consistent, physically reasonable inner potentials near $10.5 \mathrm{eV}$ while the more complex comparison in Fig. 10 leads to more unusual values.

Until a thorough investigation of the scattering potentials in the intermediate energy range is complete, the errors caused by thermal averaging, aperture integration, and inelastic mean free path may be ignored. It is obvious from the comparison of the numerical simulation in Fig. 13 that these values are not too far wrong: the overall magnitude of the oscillations is correct at high and low energy. Furthermore, the non-structural parameters may be more properly investigated by studying them directly, i.e. through temperature and aperture variations. 


\section{DISCUSSION AND CONCLUSION}

Although we are not yet in a position to quantify our accuracy, our results should be of comparable quality to other surface structure methods. The $c(2 \times 2) S / N i(001)$ system has been studied by LEED, Normal Photoelectron Diffraction (NPD), and Surface EXAFS. The $c(2 \times 2) S / N i(001)$ system served as one of the prototype surface systems for LEED so that it is inappropriate to quote much of the earlier work. The most recent results $^{21,22,4}$ agree on a $S-N i$ interlayer spacing of $d \perp 1.3 \pm 0.1$, corresponding to a $\mathrm{S}-\mathrm{Ni}$ bond length of $2.19 \pm 0.06$ A. The NPD experiment ${ }^{5}$ using the $S(2 p)$ core level gave a $S-N i$ interlayer spacing of $d_{L}=1.30 \pm .04 \AA$ ( $S-N i$ bond length of $2.19 \pm 0.03 \AA$ ). The Surface EXAFS analysis ${ }^{6}$ gave a S-Ni bond length of $2.23 \AA \pm 0.02$ \& equivalent to a $d_{L}=1.37 \pm .03 \AA$. None of these measurements addressed the $\mathrm{Ni}-\mathrm{Ni}$ interlayer spacing.

We conclude from our analysis of the two ARPEFS curves that $d_{\perp}=$ $1.31 \pm .03 \AA(\mathrm{S}-\mathrm{Ni}$ bond $2.19 \pm 0.02 \AA)$. This is in excellent agreement with the LEED and NPD results, but--if we may trust the error bars--in only fair agreement with the EXAFS analysis. Given the uncertainty we have about the scattering potential, we cannot propose to select our result over the EXAFS one, but our agreement with the NPD results is gratifying because the measurements are similar to our [001] experiment while the theoretical analysis was based on multiple-scattering calculation using a quite different approach than we have applied here, including different scattering phase shifts.

We have no comparison for the $\mathrm{Ni}-\mathrm{Ni}$ interlayer spacing of $1.83 \pm$ .03 A, or a $4 \%$ expansion compared to bulk Ni. This is equal to the expansion of the first two $\mathrm{NI}$ layers on clean $\mathrm{Ni}$ reported by Demuth and 
Rhodin $n^{23}$ but larger than the spacing, $1.78 \pm .02 \mathrm{~A}$, reported by Demuth, Marcus and Jepsen? 24 We also have some indication that $\mathrm{Ni}$ atoms in the second layer without $S$ atoms over head sink into the bulk somewhat. 


\section{REFERENCES}

1. J.J. Barton, C.C. Bahr, Z. Hussain, S.W. Robey, J.G. Tobin, L.E. Klebanoff, and D.A. Shirley, Phys, Rev. Lett. 51, 272 (1983).

2. P.A. Lee, Phys. Rev. B 13, 5261 (1976).

3. P.A. Lee and J.B. Pendry, Phys. Rev. B 11, 2795 (1975).

4. M.A. Van Hove and S.Y. Tong, J. Vac. Sci. TechnoI. 12, 230 (1975).

5. D.H. Rosenblatt, J.G. Tobin, M.G. Mason, R.F. Davis, S.D. Kevan, D.A. Shirley, C.H. Li, and S.Y. Tong, Phys. Rev. B 23, 3828 (1981).

6. S. Brennan, J. Stohr, and R. Jaeger, Phys. Rev. B 24, 4871 (1981).

7. R.J. Madon and H. Shaw, Catal. Rev.-Sci. Eng. 15, 69 (1977).

8. E.W. Plummer, B. Tonner, N. Hol zwarth, and A. Liebsch, Phys. Rev. B 21, 4306 (1980).

9. P.J. Orders, R.E. Connelly, N.F.T. Hall, and C..." adley, Phys. Rev. B 24, 6163(1981).

10. Ni etch: $30 \%$ Nitric acid, $10 \%$ sulfuric acid, $10 \%$ acetic acid, $30 \%$ glacial acetic acid, at $75^{\circ} \mathrm{C}$. The faint white cloud which sometimes accompanies this etch is easily removed in the subsequent vacuum treatment.

11. S.D. Kevan, Ph.D. Thesis, University of California, Berkeley, $(1980)$

12. Z. Hussain, E. Umbach, D.A. Shirley, J. Stöhr, and J. Feldhaus, Nucl. Inst. Methods 195, 115 (1982).

13. J.J. Barton, S.W. Robey, D.A. Shirley, "Theory of Angle-Resolved Photoemission Extended Fine Structure", LBL-19324, and Chapter 5.

14. J.J. Barton and D.A. Shirley, "Fourier Analysis of Extended Fine Structure with Autoregressive Prediction", LBL-14758, and Chapter 6. 
15. E.0. Brigham, The Fast Fourier Transform, Prentice-Hall, Englewood Cliffs, N.J. 1974.

16. M. Sagurton, E.L. Bullock, and C.S. Fadley, Phys. Rev. B $\underline{30}, 7332$ (1984).

17. S.Y. Tong and C.H. Li, in Chemistry and Physics of Solid Surfaces, v. III. ed. R. Vanselow, England, (1982), p. 287.

18. J.J. Barton and D.A. Shirley, "A Generalized Ramsauer-Townsend Resonance in ARPEFS Oscillations", LBL-19325 and Chapter 8.

19. B.K. Teo and P.A. Lee, J. Am. Chem. Soc. 101, 2815 (1979).

20. P.A. Lee, P.H. Citrin, P. Eisenberger, and B.M. Kincaid, Rev. Mod. Phys. 53, 769 (1981).

21. Y. Gauthier, D. Aberdam, R. Baudoing, Surf. Sci. 프, 339 (1978).

22. J.E. Demuth, D.W. Jepsen, and P.M. Marcus, Phys. Rev. Lett. 31, 540 (1973).

23. J.E. Demuth and T.N.Rhodin, Surf. Sci. 42, 261 (1974).

24. J.E. Demuth, P.M. Marcus, and D.W. Jepsen, Phys. Rev. B 11, 1460 (1975). 


\section{FIGURE CAPTIONS}

Figure 1. Inelastic scattered electron background spectrum for $h v=2504 \mathrm{eV}$ and, inset, a sample least squares fit to a $S(1 \mathrm{~s})$ photoemission peak. The small features near $174 \mathrm{eV}$ in the background spectrum are 5 Auger peaks. In the Inset, the solid circles are the measure photoemission counts, the upper solid curve gives the fitted function values, while the lower solid curve is the sum of the smoothed background spectrum and the error function step. The photopeak area is the area between the solid curves.

Figure 2 Normalized angle-resolved photoemission intensities as a funciion of photopeak energy and atomic-like $I_{0}$ curves from least squares $f$ its for $c(2 \times 2) S / N 1(001)$. Solid curve is the photoemission intensities, and the dotted curve is the $I_{0}$ estimate. (a) Emission along, [011], (b) Emission along [001]

Figure 3 ARPEFS oscillation for $c(2 \times 2) S / N i(001)$ versus electron kinetic energy. (a) [011] emission (b) [001] emission.

Figure 4 ARPEFS oscillations after conversion to a momentum scale using an inner potential of $10.5 \mathrm{eV}$, and after interpolation to an even mesh of 128 points. (a) Emission along, [011], (b) Emission along [001] 
Figure 5 Fourier transform magnitudes versus scattering path length difference for $k$ times the data in figure $3 a$. In the lower panel, the conventional Fourier transform was applied, while the upper panel was obtained with the auto-regressive linear prediction method described in ref. 14 .

Figure 6 Fourier transform magnitudes versus scattering path length difference for $k$ times the data in $\mathrm{f}$ igure $3 \mathrm{~b}$. In the lower panel, the conventional Fourier transform was applied, while the upper janel was obtained with the auto-regressive linear prediction method described in rep. 14.

Figure 7 Contour map of the least square error for fits of numerical simulations to liata Fourier filtered at 5.1A, from the [011] emission experiment.. The horizontal axis gives the spacing along the crystal normal between the sulfur photoemitter and the first layer of $\mathrm{N} 1$ atoms $\left(d_{1}\right)$. The vertical axis gives the variation of the polar angle of emission measured from the surface normal. Inner potential fixed at $10.5 \mathrm{eV}$

Figure 8 Contour map of the least square error for fits of numerical simulations to Fourier filtered data from the [001] emission experiment. The horizontal axis gives the spacing between the first layer of $\mathrm{Ni}$ atoms and those 2nd layer $\mathrm{N} 1$ atoms having $\mathrm{S}$ overlayer atoms on top of them (Atopped atoms). The vertical axis gives the same quantity for 2nd layer $\mathrm{Ni}$ atoms having no $S$ overhead (open atoms). The dot-dashed line follows the 
constralned coplanar geometry. Inner potential fixed at $10.5 \mathrm{eV}$

Figure 9 Contour map of the least square error for fits of numerical simulations to Fourier filtered data from the [011] emission experiment. The horizontal axis gives the spacing between the first layer of $\mathrm{Ni}$ atoms and those 2nd layer $\mathrm{Ni}$ atoms having $\mathrm{S}$ overlayer atoms on top of then (Atopped atoms). The vertical axis gives the same quantity for 2nd layer $\mathrm{Ni}$ atoms having no S overhead (open atoms). The dot-dashed line follows the constrained coplanar geometry. Inner potential fixed at $10.5 \mathrm{eV}$

Figure 10 Contour map of the least square error for fits of numerical simulations to data for the [001] emission experiment. The horizontal axis gives the spacing between the first layer of $\mathrm{N} 1$ atoms and those 2nd layer $\mathrm{N} 1$ atoms having $\mathrm{S}$ overlayer atoms on top of them (Atopped atoms). The vertical axis gives the same quantity for 2nd layer $\mathrm{Ni}$ atoms having no $\mathrm{S}$ overhead (open atoms). The dot-dashed line follows the constrained coplanar geometry. The inner potential and overall scale of the theory was fitted to the data.

Figure 11 Refinement of the S N1 Interlayer spacing with unfiltered data curves and a fixed $S$ to 2nd layer spacing of 3.135 A. The upper curves are from the [011] experiment and the bottom pair are from the [001] experiment. The solid curves have a fixed 
inner potential for theory of $10.5 \mathrm{eV}$, while the dashed curves correspond to varying the scale and inner potential of the theory to best fit the data.

Figure 12 Comparision of Fourier filtered (5.1A) ARPEFS oscillations from the [011] experiment (solid circles), the numerical simulation for $d_{\perp}=1.30$ A (solid line), and the numerical simulation for $\mathrm{d}_{L}=1.35 \mathrm{~A}$ (dashed curve)

Figure 13 Comparision of Fourier filtered (10.5A) ARPEFS oscillations from the [011] experiment (solid curve) to the numerical simulation for $d_{\perp}=1.30 \mathrm{~A}$ and a $S$ to first $\mathrm{Ni}$ layer spacing of 1.84A (dashed curve). The atopped and open $\mathrm{Ni}$ atoms are coplanar in the theory curve.

Figure 14 Comparision of Fourier filtered (10.5A) ARPEFS oscillations from the [011] experiment (solid curve) to the numerical simulation for $d_{\perp}=1.30 \mathrm{~A}$ and a $S$ to first $\mathrm{Ni}$ layer spacing of 1.76月 (dashed curve) (the bulk interlayer spacing is 1.76 A). The atopped and open Ni atoms are coplanar in the theory curve. 


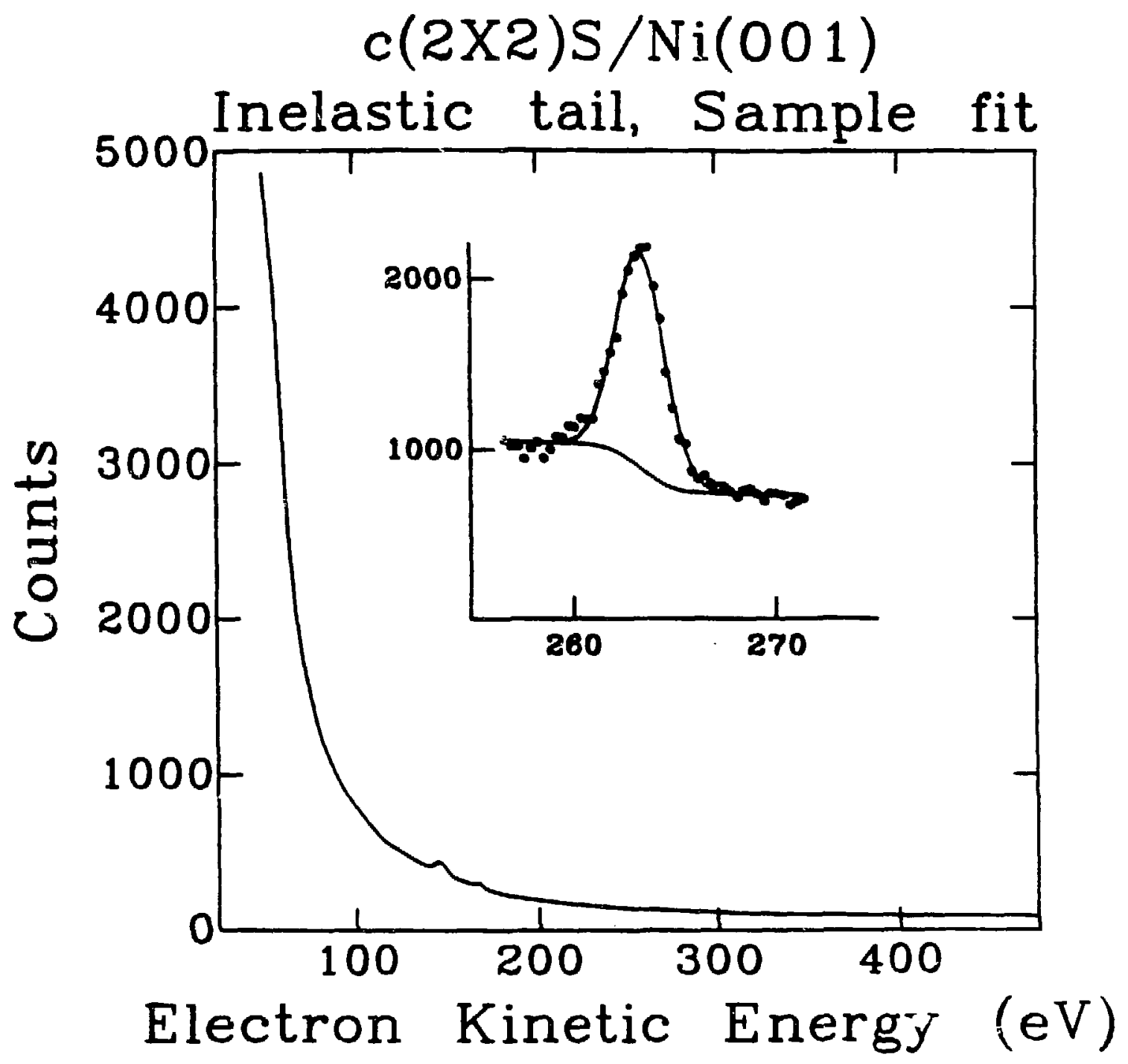

XBL 853-1833

Figure 1 
c(2X2)S/Ni(001)
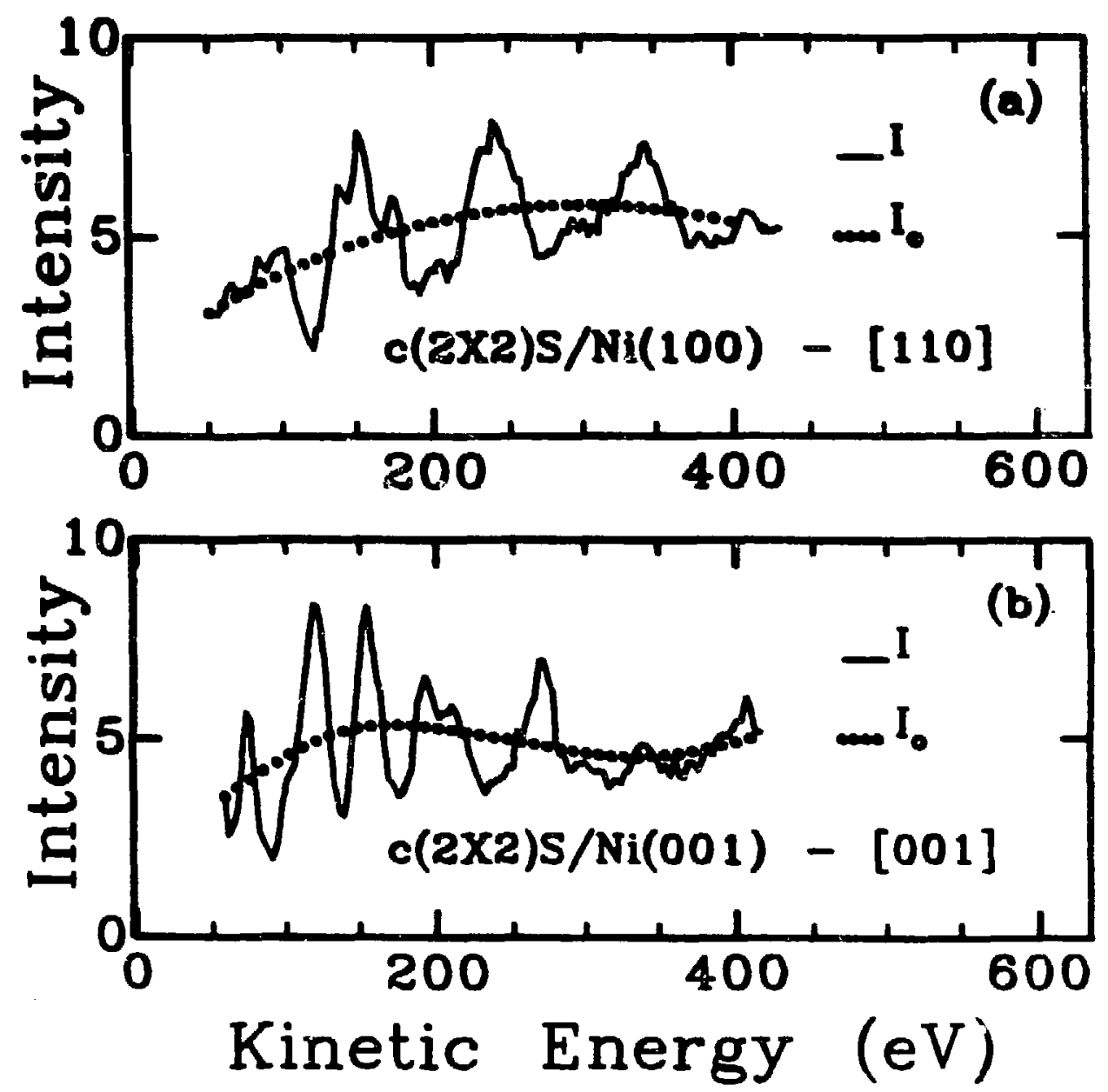

XBL 853-1825

Figure 2 


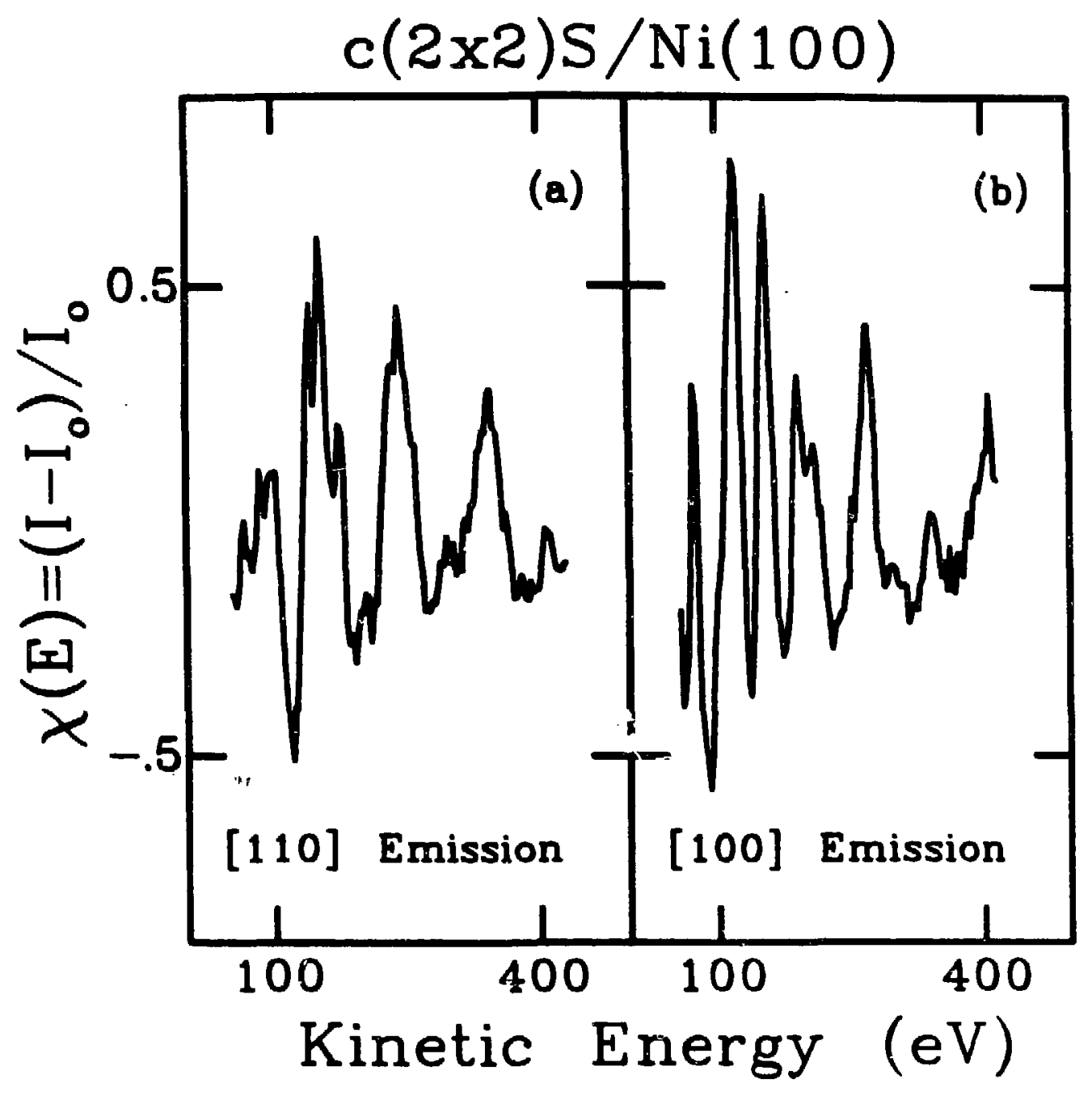

XBL 848-3546 

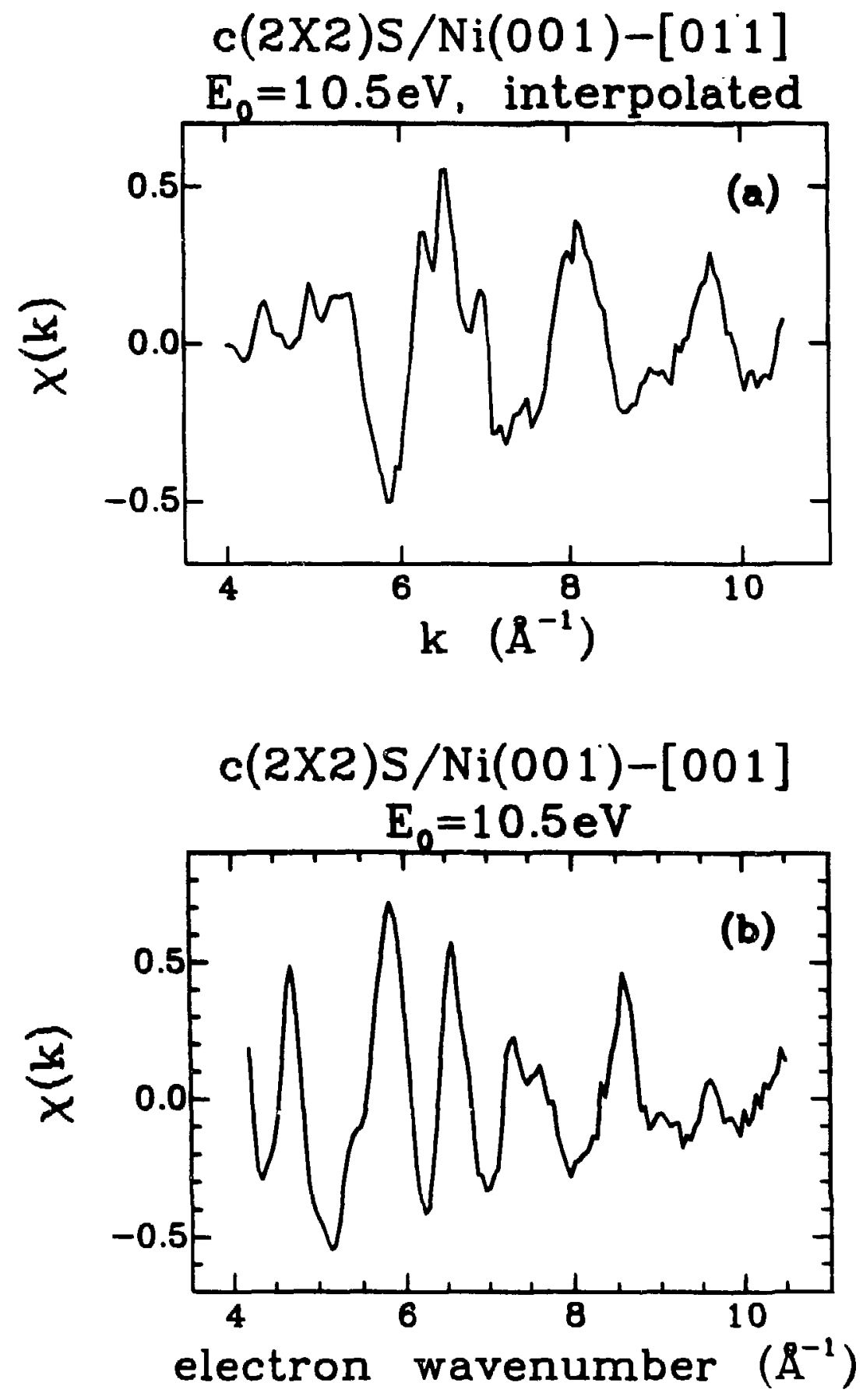

Y.BL 853-1824

Figure 4 
$c(2 X 2) S / N i(0001)-\left[\begin{array}{lll}0 & 1 & 1\end{array}\right]$

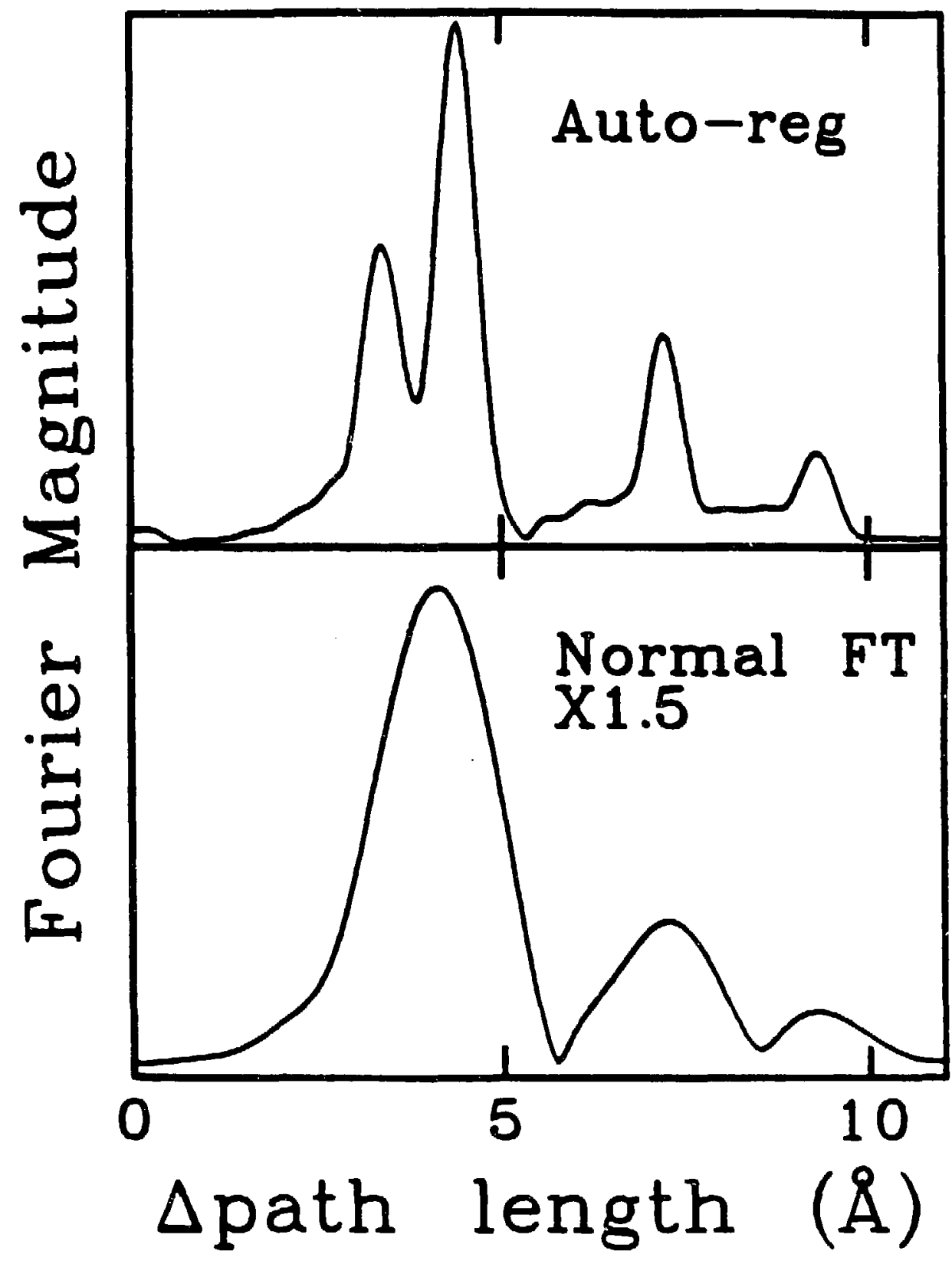

XBL 853-1835

Figure 5 
$\mathrm{c}(2 \mathrm{X} 2) \mathrm{S} / \mathrm{Ni}(001)-[001]$

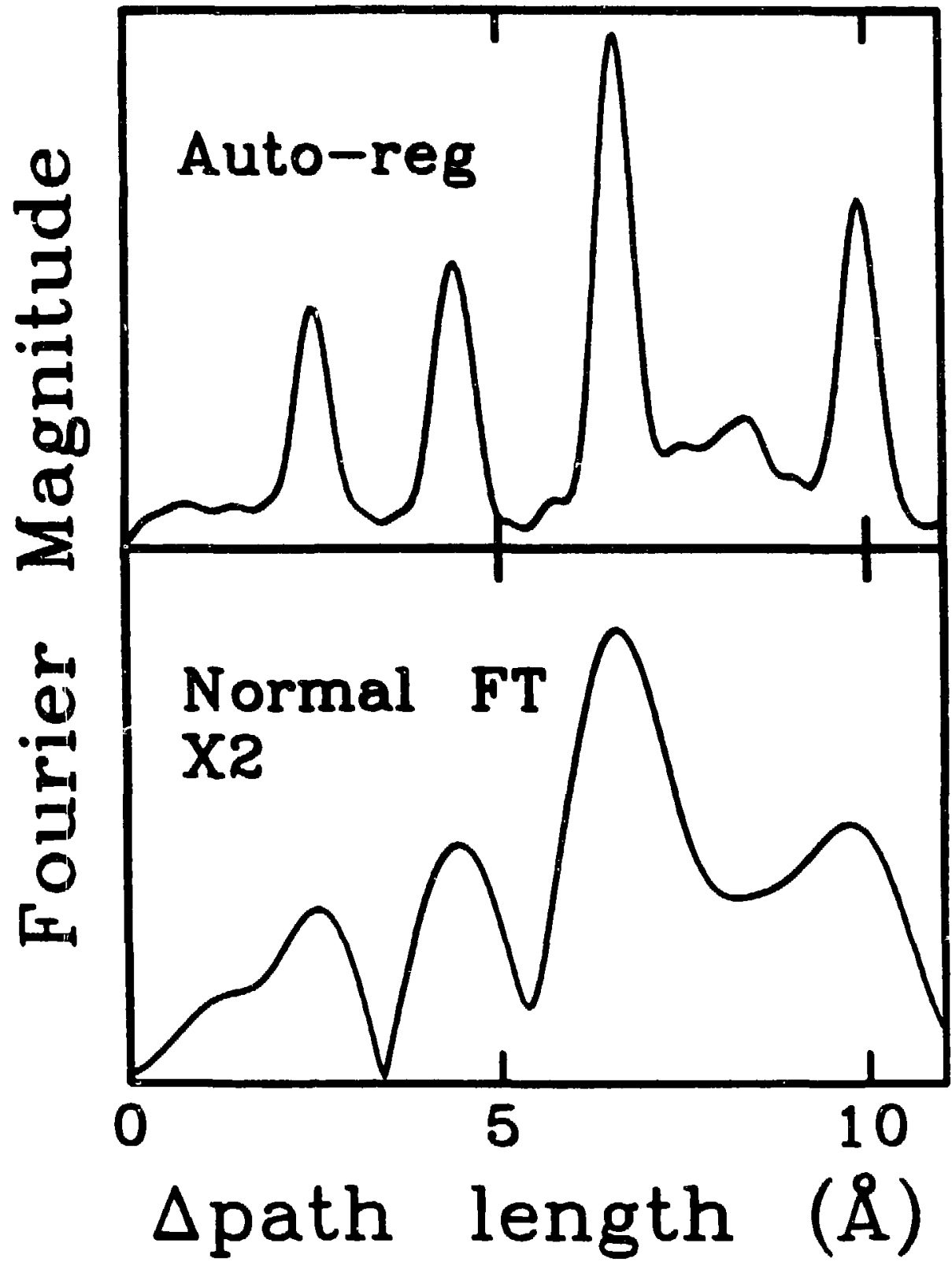

XBL 853-1836

Figure 6 


$$
\text { c(2X2)S/Ni(001)-[0 } 011 \text { 1] }
$$

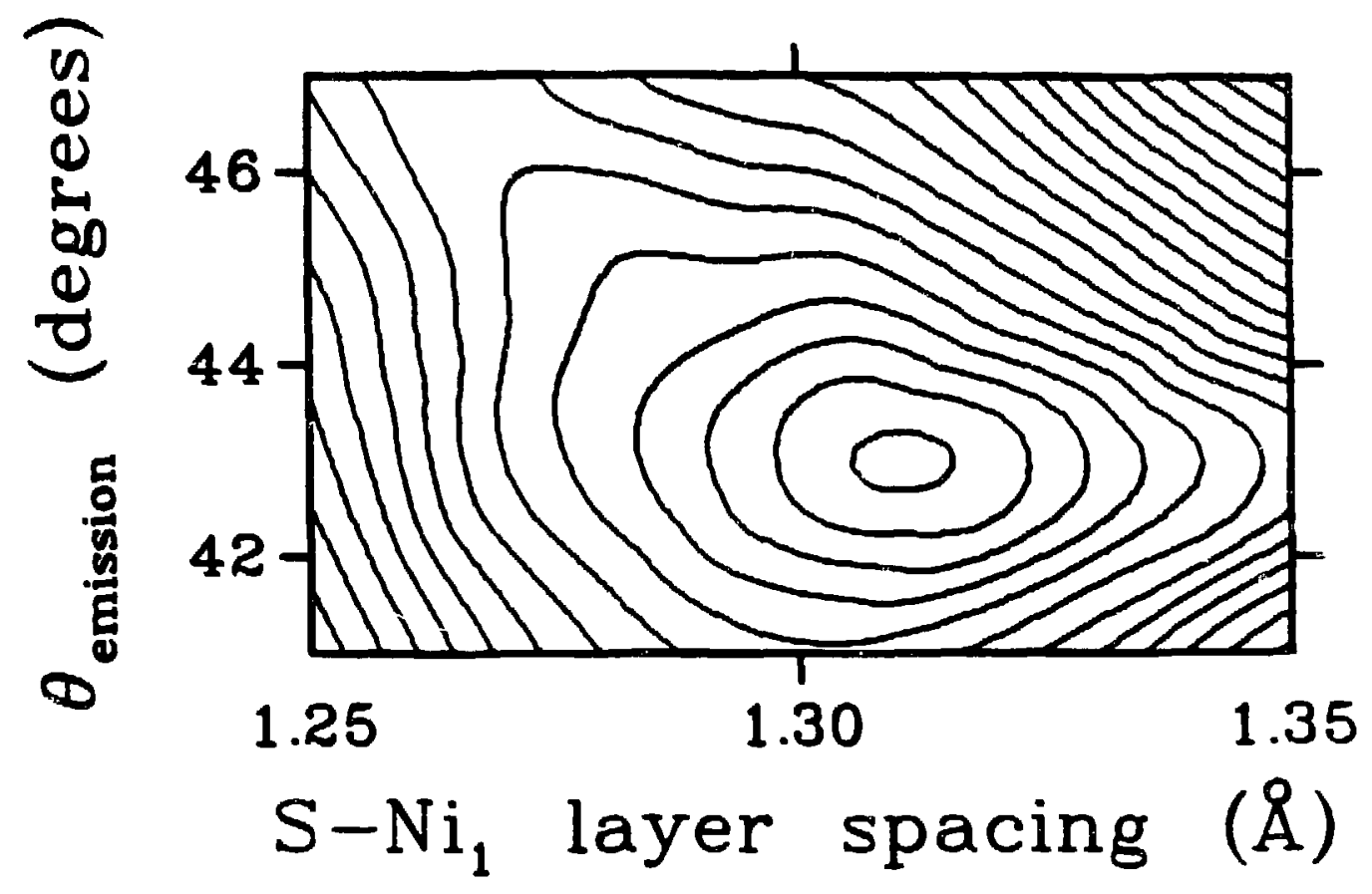

XBL 853-1841 


\section{$\mathrm{c}(2 \mathrm{X} 2) \mathrm{S} / \mathrm{Ni}(001)-\left[\begin{array}{lll}0 & 0 & 1\end{array}\right]$}

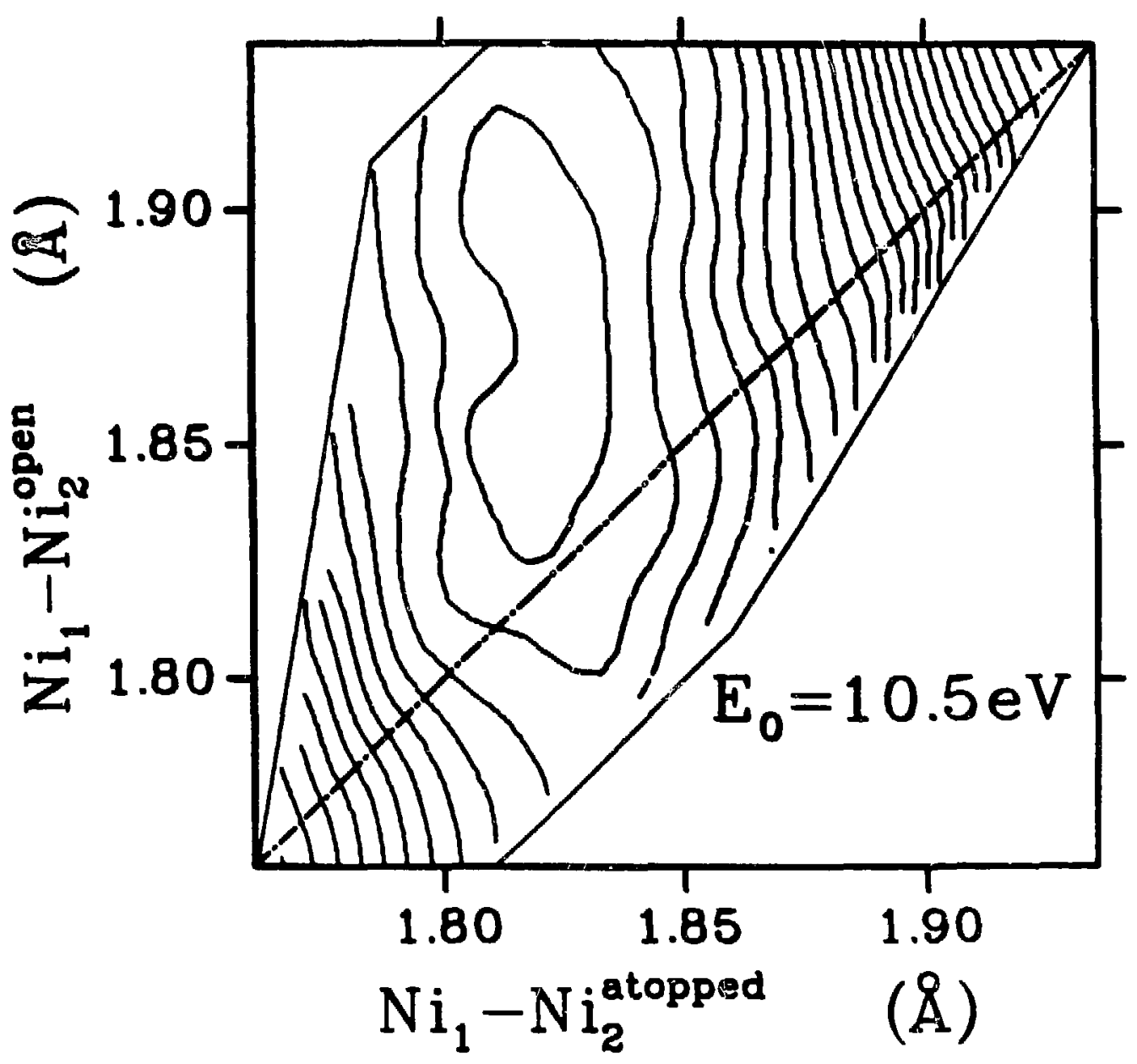

XBL 853-1823

Figure 8 
$\mathrm{c}(2 \mathrm{X} 2) \mathrm{S} / \mathrm{Ni}(001)-\left[\begin{array}{lll}0 & 1 & 1\end{array}\right]$

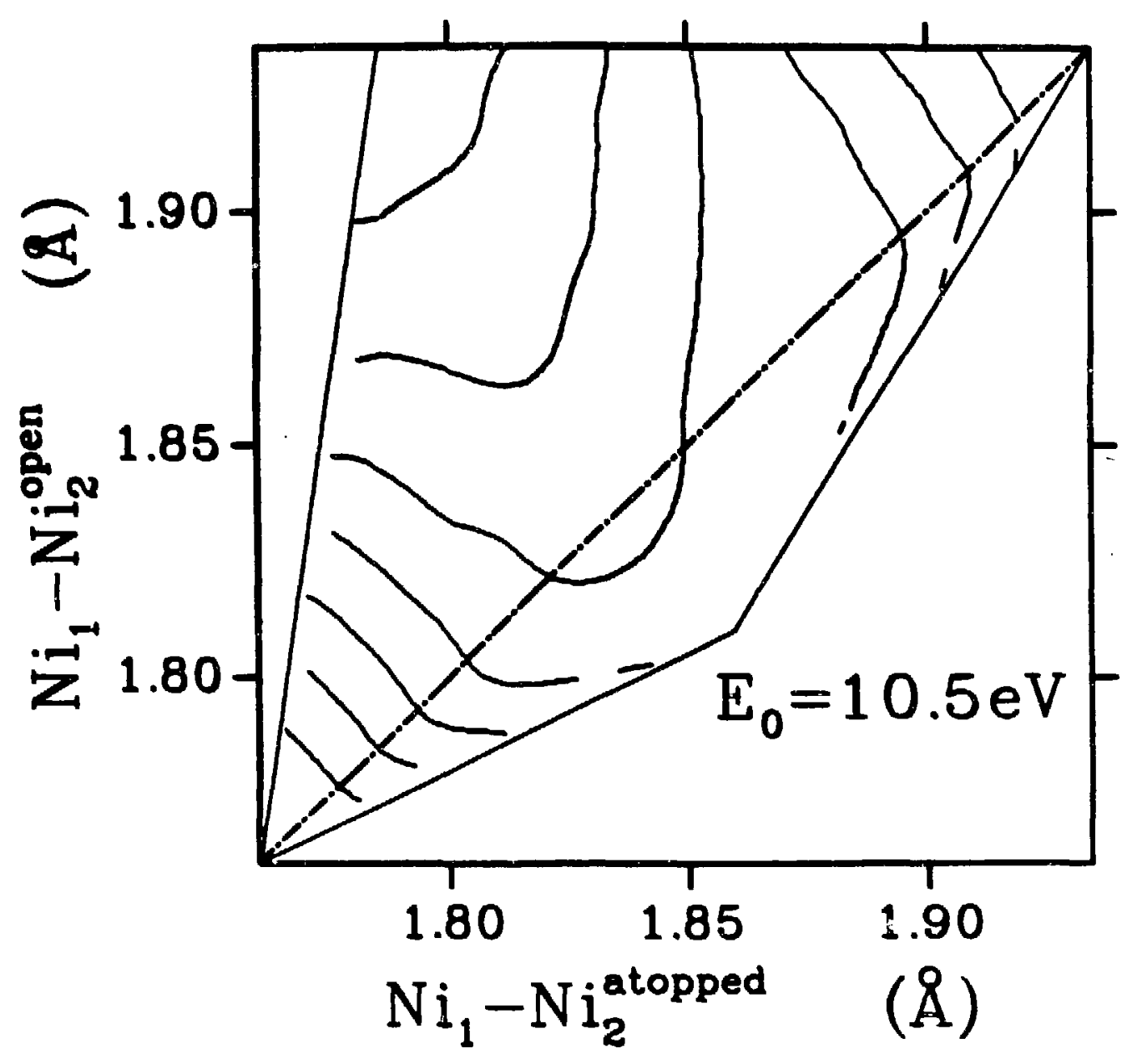

XBL 853-1817 
$\mathrm{c}(2 \mathrm{X} 2) \mathrm{S} / \mathrm{Ni}(001)-[001]$

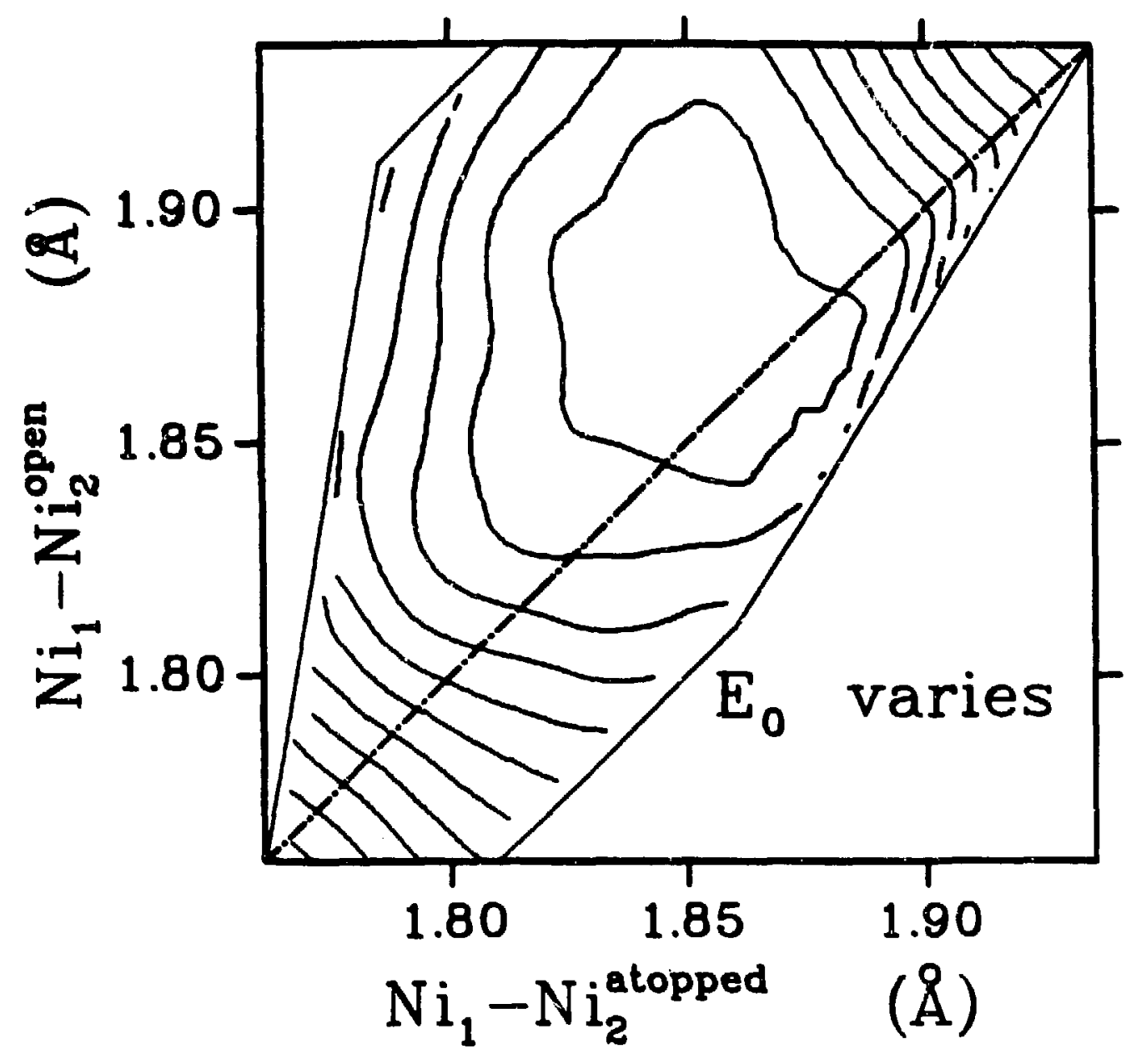

XBL 853-1842

Figure 10 


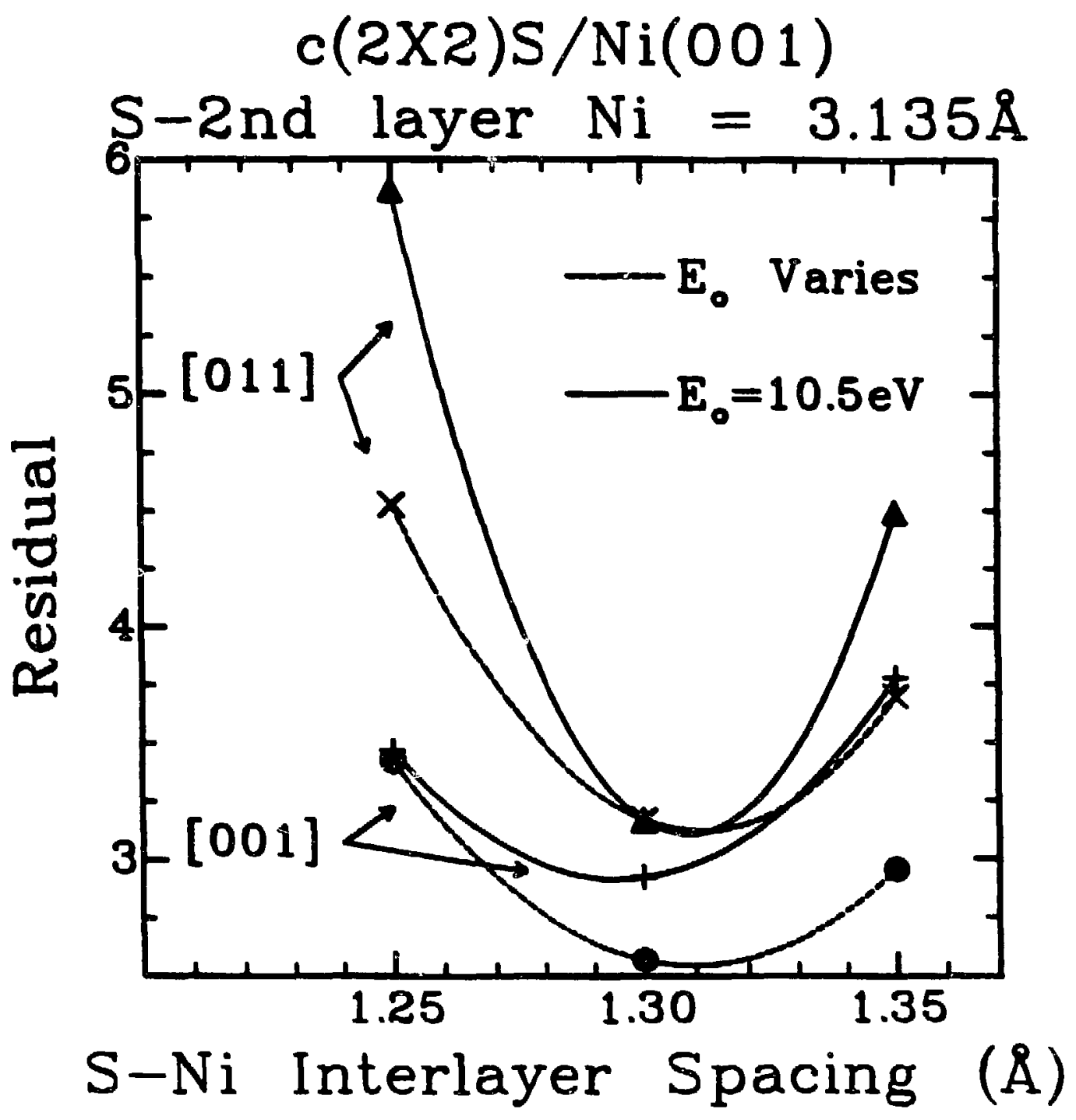

XBL 853-1834

Figure 11 


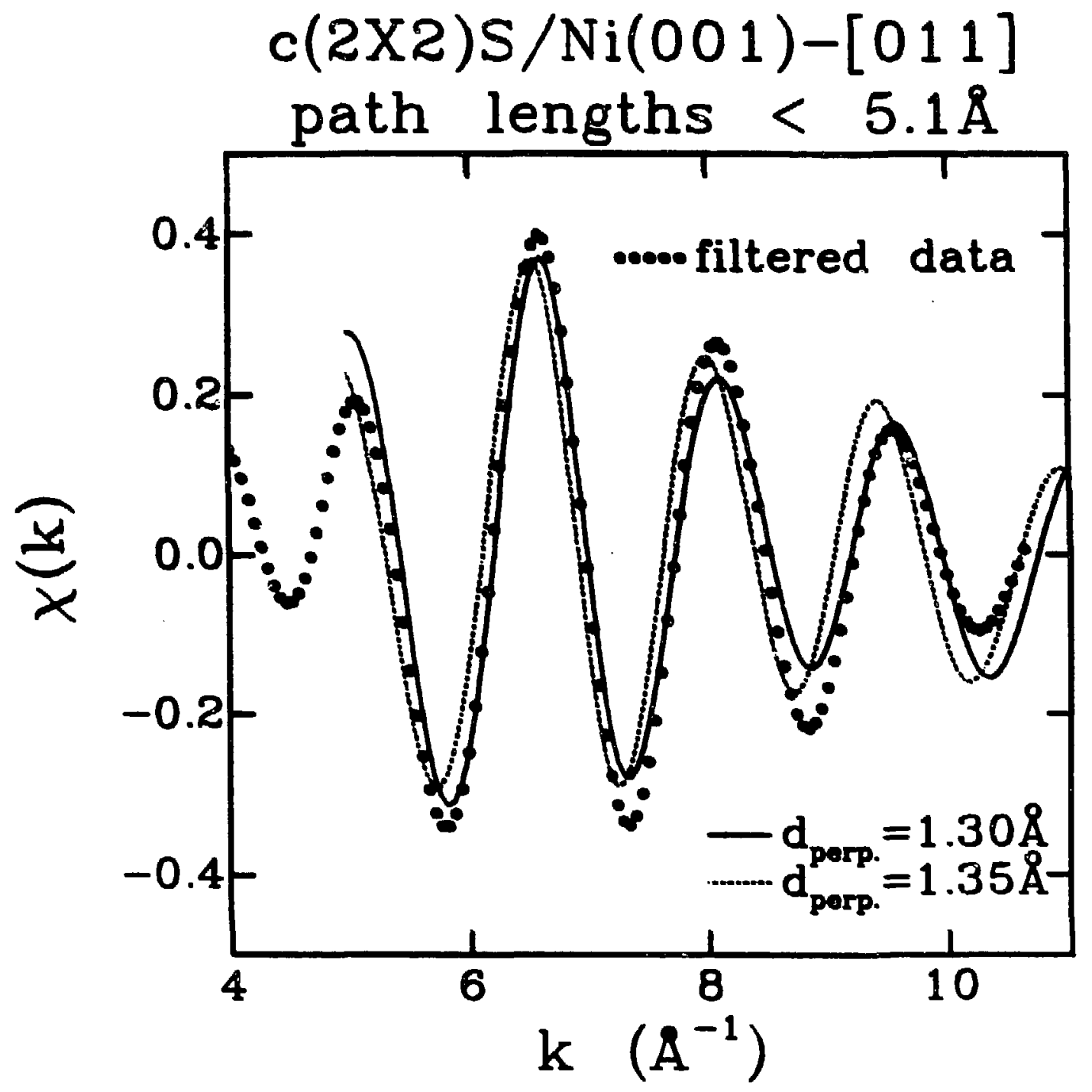

XBL 853-1822

Figure 12 


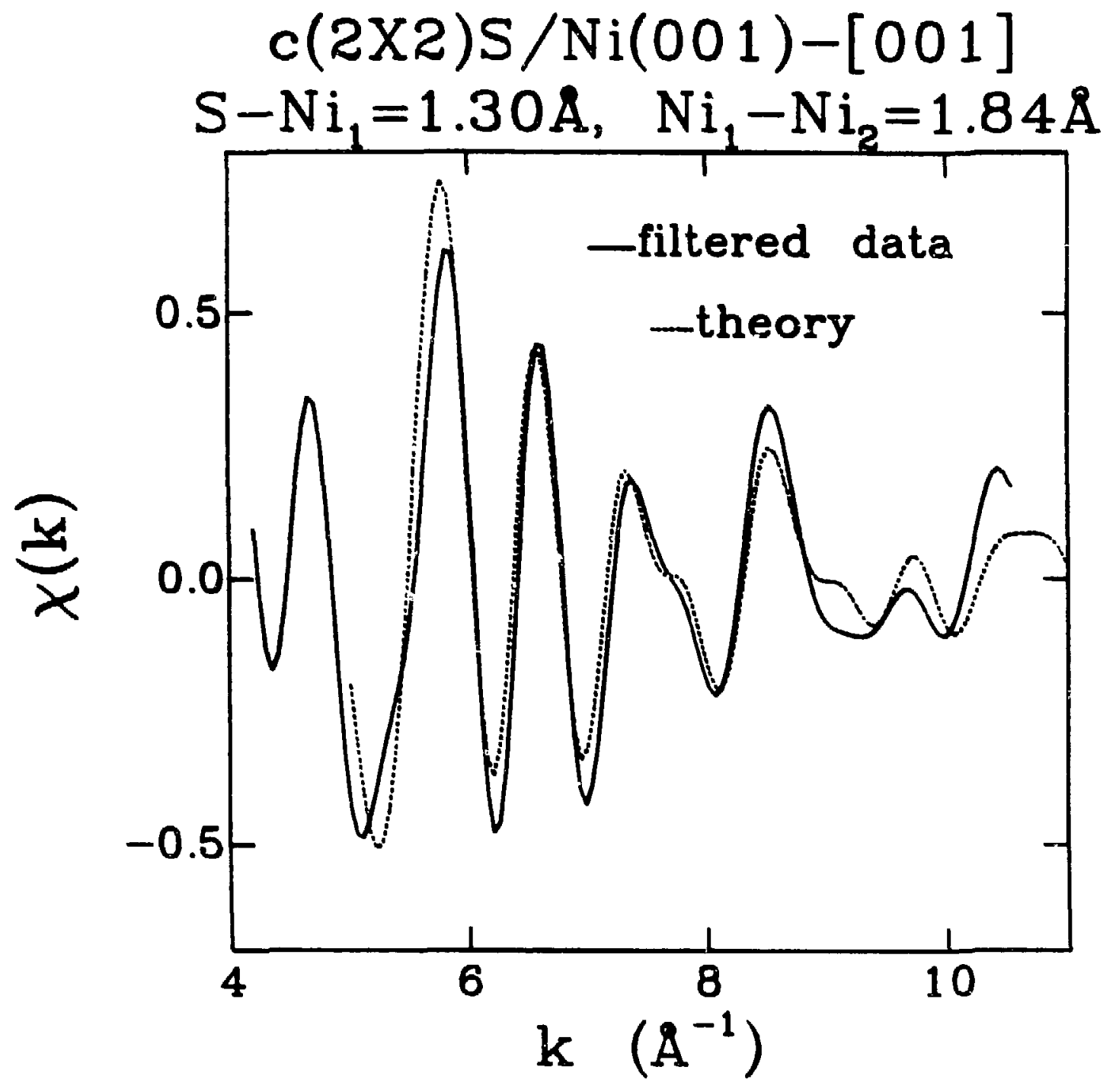

XBL 853-1821

Figure 13 


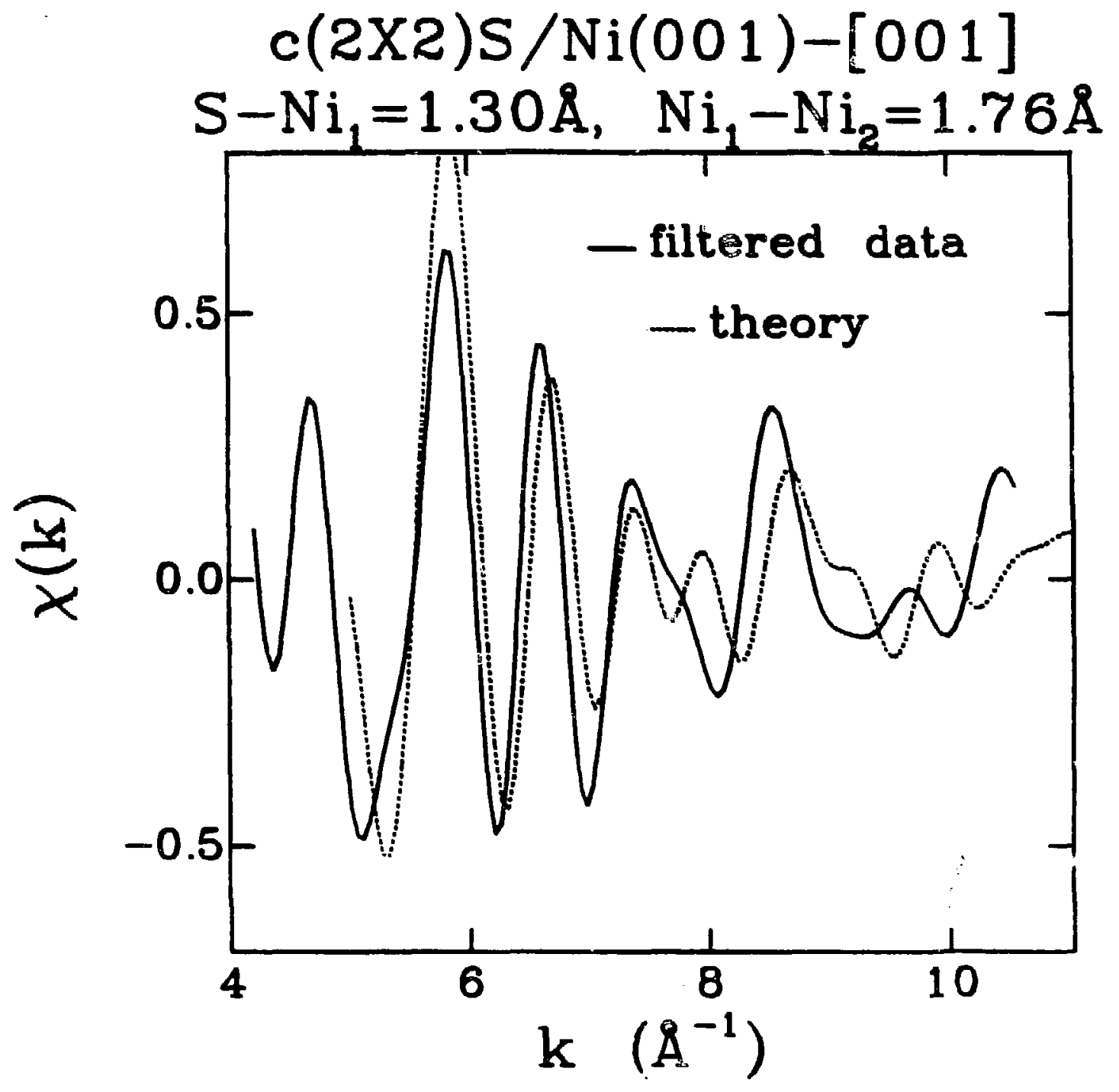

$X\ulcorner L$ 853-1819

Figure 14 
CHAPTER 8:

A GENERALIZED RAMSAUER-TOWNSEND RESONANCE IN ARPEFS OSCILLATIONS

\section{ABSTRACT}

We observe a resonance in the scattering amplitude for $\mathrm{S}(1 \mathrm{~s})$ photoelectrons from $\mathrm{Ni}$ atoms and interpret this resonance as a dip in the $\mathrm{Ni}$ atom partial cross section for electron scattering related to the Ramsauer-Townsend effect. This generalized Ramsauer-Townsend effect occurs at a particular energy and angle rather than in the total elastic cross section. We show that the resonance energy is sensitive to curved-wave corrections and, after including multiple-scattering effects, we derive the S-Ni bond length in $\mathrm{c}(2 \times 2) \mathrm{S} / \mathrm{Ni}(100)$ from the ARPEFS oscillations from nearest neighbor $\mathrm{Ni}$ atoms in the presence of the generalized Ramsauer-Townsend resonance. We find this bond length to be $2.20 \mathrm{~A} \pm .03 \mathrm{~A}$ corresponding to a S-Ni interplanar distance (d $)$ of $1.32 \pm .04 \mathrm{~A}$. 


\section{INTRODUCTION}

The nature of the sore-level photoemission inten_ity oscillations known as photoelectron diffraction has been substantially clarified by new theoretical ${ }^{1,2}$ and experimental work which shows that these oscillations--caused by interference between direct and scattered photoemission probability amplitude ${ }^{3,4}--$ are qualitatively predicted by scattering path-length differences. A complete understanding of the physics of these oscillations has important consequences for the use of photoelectron diffraction as a technique for determining surface structure: the total scattering path-length difference is the sum of a geometrical path length and an ion-core potential phase shift function, allowing the geometry to be deduced if the potential can be adequately modeled. While most of the energy dependent photoelectron diffraction measurements 5,6 have been made in the kinetic energy range: from 20-150 eV, we have recently ${ }^{7,8}$ been concentrating on photoelectron energies between 100-600 eV. These intermediate energies and the wider energy range are advantageous if we wish to concentrate on structure determination because the potential phase shift functions are less

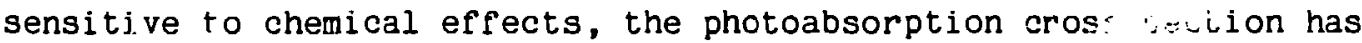
less struci:e, and the pho. llectron scattering partial cl uss section is more anisotropic giving a simpler and more structure sensitive spectrum. For very similar reasons the $x$-ray absorption fine structure spectroscopies have been divided into $x$-ray absorption near edge structure (XANES or NEXAFS) at low energies and extended $x$-ray absorption fine structure (EXAFS) in the intermediate 100-1000 eV range. We refer to the core-level angle-resolved photoemission measurements in 
the 100-1000 ev, intermediate energy range as angle-resolved photoemission extended $f$ ine structure (ARPEF).

To concentrate on the oscillating, non-atomic signal we remove the slowly varying part, $I_{0}$, of the intensity, $I$, to form $x=\left(I-I_{0}\right) / I_{0}$. Every model for ARPEFS predicts that the oscillations, expressed as a furction of electron wave number, $k$, are cosinusoidal:

$x(k)=\sum_{j} A_{j}(k) \cos \left[k r_{j}\left(1-\cos \theta_{j}\right)+\phi_{j}(k)\right]$

where $A_{j}(k)$ is an amplitude function, $\phi_{j}$ is a scattering potential phase function, and $\left(r_{j}-r_{j}, \cos \theta_{j}\right)$ is the geometrical path-length difference for bond length $r_{j}$ and scattering angle $\theta_{j}$. The sum on $j$ extends over all atoms near the photoemltter which have significant amplitude. In the simplest case, the amplitude function is large for only a few atoms and has little structure as a function of $k$, and the phase similarly benign. Then the ARPEFS curve may be Fourier analyzed and--provided Fourier resolution is adequate--the Fourier amplitude spectrum should have peaks corresponding scattering path-length differences. ${ }^{7}$ If, on the other hand, the amplitude function is not a smooth envelope, the Fourier spectrum for the corresponding scattering event will not peak at the scattering path-length difference. In this paper we investigate structure determination with ARPEFS in the case that the scattering potential for nearest neighbor scattering atom has a strong amplitude dependence caused by an interesting resonance related to the RamsauerTownsend effect.

We have selected $c(2 \times 2) S / N i(100)$ for our study primarily because the $\mathrm{S}$ adsorption site and the $\mathrm{S} / \mathrm{Ni}$ bond length have been reported 
previously and the overlayer can be reproduced easily. Elsewhere we report ${ }^{9}$ a study of the $S(1 s)$ ARPEFS from $c(2 \times 2) S / N i(100)$, concentrating on the scattering events which have Fourier peaks near the scattering path-length difference. Here we will discuss normal emission ARPEFS from the nearest neighbor $\mathrm{N} I$ atoms in the four-fold hollow site. The amplitude for the scattering of $\mathrm{S}$ photoelectrons from $\mathrm{Ni}$ potentials into the normal direction dips sharply as the energy is scanned leading to a spit Fourier peak. 


\section{EXPERIMENTAL}

We have given a thorough discussion of our measurement procedure in ref 9 so we will be brief here. Our sample was prepared in a standard fashion: a mirror-finish, oriented Ni(001) single crystal was cleaned in vacuum, exposed to $\mathrm{H}_{2} \mathrm{~S}(\mathrm{~g})$, and heated briefly to $200^{\circ} \mathrm{C}$ to give the $c(2 \times 2) S / N i(100)$ LEED pattern. This sample was illuminated by soft $x-$ rays from the Stanford Synchrotron Radiation Laboratory Jumbo monochromator ${ }^{10}$ and $S(1 s)$ angle-resolved photoemission intensity spectra were measured every $3 \mathrm{eV}$ for photon energies between 2532 and $2950 \mathrm{eV}$. Assuming that the $x$-rays are completely polarized in the plane of the synchrotron storage ring, we oriented the $\mathrm{Ni}$ crystal to place the electric vector of the light, $\hat{E}, 30^{\circ}$ from the surface normal toward a [110] direction and rotated the angle-resolved electron energy analyzer to collect spectra along the surface normal. The individual photoemission measurements were reduced to partial cross-section measurements ir the fashion described in ref. 9.

The resulting ARPEFS curve $x(k)$ is given in Fig. 1. The energy scale has been converted to a wavenumber scale using an inner potential of $10.5 \mathrm{eV}$. The curve is seen to be dominated by an oscillation with 6 cycles in $2 \pi \AA^{-1}$, corresponding to an interference path length of $6 \AA$. Since we know the nearest neighbor bond length in this system is -2.2 A corresponding to a maximum path-length difference of $4.4 \AA$, we can conclude that the nearest neighbor scattering does not dominate this curve.

Fourier transforms of this curve are shown in Fig. 2. For the upper panel, Fig. 2a, we have multiplied $k \chi(k)$ by a Gaussian of full width half maximum of $4 \AA^{-1}$ centered on the data range, added zeroes to 
fill 2048 cells and applied the fast Fourier transform; the Fourier transform magnitude is plotted versus path-length difference. In the lower panel the Autoregressive Fourier method described in ref 11 has been applied to $k_{\chi}(k)$ and the resulting extrapolated sequence has been multiplied by a Gaussian of $12 \mathrm{~A}^{-1}$ full width half maximum.

The peaks in the Fourier spectrum above 5 \& path-length difference have been discussed in ref. 9. The path-length difference derived from the positions of these Fourier peaks is approximately equal to the scattering path-length difference for backscattering $\mathrm{Ni}$ atoms in the second and third Ni layers. The positions of the two peaks below 5 a do not correspond to any path-length difference, and no feature in the Fourier spectrum appears near 3.5 \& where we would expect a scattering path-length due to $\mathrm{Ni}$ nearest neighbors (assuming a four-fold hollow adsorption and reasonable $\mathrm{S}-\mathrm{Ni}$ bond distances). The physical origin of these peaks is the subject of this paper. 


\section{GENERALIZED RAMSAUER TOWNSEND RESONANCE}

The lack of correspondence between the scattering path-length difference for nearest neighbor $\mathrm{Ni}$ atoms and Fourier spectrum peaks is caused by a strong dip in the scattering power for $\mathrm{Ni}$ as a function of energy which occurs for scattering angles near those appropriate for normal emission from $c(2 \times 2) \mathrm{S} / \mathrm{Ni}(001)$. This dip is related to the wellknown Ramsauer-Townsend electron scattering resonance, and hence we call this amplitude effect a generalized Ramsauer-Townsend (GRT) reson:nce. Ramsauer and Townsend observed that argon becomes transparent to electrons at $0.7 \mathrm{eV}$. The origin of this surprising lack of scattering, suggested by N. Bohr and verified by Faxen and Holtsmark, ${ }^{12}$ follows from the partial-wave formula for the (complex) scattering amplitude for electrons :

$f(\theta, k)=\frac{1}{2 i k} \sum_{\ell=0}(2 \ell+1) P_{\ell}(\cos \theta)\left(e^{2 i \delta} \ell-1\right)$

where $\delta_{\ell}(k)$ are the ion-core partial-wave phase shifts. At very low kinetic energies only $\delta_{0}$, the isotropic $s$ wave, contributes to the scattered wave. If, as is the case for Ar at $0.7 \mathrm{eV}$, the value of the $\mathrm{S}$ phase shift were exactly $180^{\circ}$, then even this term goes to zero and the amplitude, $f(\theta, k)$ becomes very small for all angles.

Our generalized Ramsauer-Townsend effect is more complicated. ${ }^{13}$ at higher energies many partial waves contribute to the scattering amplitude. Then only with the proper linear combination of angular factors, $(2 \ell+1) P_{\ell}(\cos \theta)$, and energy factors, $\left(e^{2 i \delta_{\ell}}-1\right)$, will the 
scattering amplitude be zero. Thus while the classical RamsauerTownsend effect gives zero amplitude at all angles and very low energy based on special behavior by one phase shift, our generalized RamsauerTownsend effect occurs at special angles and energies with many phase shifts involved.

Some ideas about the consequences of this effect can be derived from Fig. 3. Scattering amplitudes for four different angles are plotted in the complex plane. For each angle a line represents $f\left(k, \theta_{j}\right)$ for $k=4 \mathrm{~A}^{-1}(60 \mathrm{eV})$ to $k=12 \mathrm{~A}^{-1}(550 \mathrm{eV})$. The distance from the origin to a point on the line represents the scattering power for that angle and energy; the angle from the positive real axis to that point gives the wave phase shift caused by the potential.

For $\alpha_{j}=180^{\circ}$ we see the scattering power peaks broadly around $k=$ $6 A^{-1}$. From $k=4 A^{-1}$ to $k=12 A^{-1}$ the phase angle sweeps gently through $-60^{\circ}$. For $\theta_{j}=130^{\circ}$, however, the behavior is radically different. Now the scattering amplitude approaches the origin for $k=$ $8 \mathrm{~A}^{-1}$. The amplitude falls nearly to zero here, and the phase angle sweeps rapidly through $180^{\circ}$. The behavior for $\theta_{j}=125^{\circ}$ is similar, but the phase angle is rotating in the opposite direction.

Clear evidence that the generalized Ramsauer-Townsend effect is responsible for splitting the Fourier peak expected near $3.5 \mathrm{~A}$ into the two peaks actually observed in the Fourler spectrum is obtained by backtransforming just those Fourier coefficients whose frequencies are less than 5 A. As shown in Fig. 4, the resulting filtered ARPEFS curve shows a beat pattern consistent with a $3.5 \mathrm{~A}$ oscillation wich a superimposed amplitude envelope which dips at $k=7.5 \mathrm{~A}^{-1}$. If the phase shift function is extracted from the filtered data, ${ }^{14}$ it exhibits the 
phase jump of $\pi$ characteristic of the GRT zero crossing as shown in Fig. 5.

In our initial attempts to use the $\mathrm{Ni}$ GRT resonance, ${ }^{15}$ we sought to compare the observed phase jump to calculated $\mathrm{Ni}$ atom phase shift functions. As is evident from a comparison of the experimental phase function to the theoretical phase function calculated in the plane-wave approximation (see Fig. 5, long dashed curve), the resonance position in energy and angle is not correctly placed in this simple model. We therefore introduced curved wavefront corrections ${ }^{16}$ which, as the remaining curves in FIg. 5 demonstrate, places the calculated resonance of the experimentally observed energy. That the resonance is sensitive to the wavefunction calculation is not surprising given that several large partial-wave amplitudes must sum to zero at resonance: any slight error in the weighting of these waves will shift the resonance position. It would appear from Fig. 5 that we may assign the S-Ni bond length by comparing the observed phase shift function shape to calculated functions which include the curved-wave corrections. The strong dependence of the GRT resonance on scattering angle would set a firm limit on the bond distance, and we would have an elegant method to estimate the surface bond angle. ${ }^{8}$ However, this does not allow for the possibility of multiple scattering, and in view of the sensitivity of the resonance we must include this effect.

Fortunately, the calculation we require is very modest: we need only 20 scattering paths. The first four are the single scattering paths from the four NI nearest the photoemitting S, with path lengths near $3.5 \mathrm{~A}$. The single-scattering wave from the nearest neighbors can double scatter from either of two atoms in the $S$ layer, giving a total 
of eight more paths near $4.4 \AA$. We also include scattering from four $S$ atoms at $3.52 \AA$ and four more at $4.98 \AA$ even though the signal from these atoms is very small. Indeed, without curved-wave corrections ${ }^{16}$ these $S$ atoms would cancel in pairs: the phase of the direct wave incident upon one member of the pair is opposite the phase for the other. Of the 20 possible paths possible, only ten are unique, and by employing the method of ref 2 , the calculation requires no more effort than other steps in the data analysis process.

The resulting multiple-scattering phase functions are shown in Fig. 6. The multiple-scattering effect is small, but it is adding to a near zero signal. We find that the GRT resonance has been pushed up in angle from $127.5^{\circ}$ in single scattering to $131^{\circ}$ in multiple scattering. Fig. 6 shows that the phase jump for $131.4^{\circ}$ is on the opposite side of the origin from the experimental jump, setting an upper bound on the $\mathrm{S}-\mathrm{Ni}$ interlayer spacing, $d_{\perp}$ of $1.50 \AA$ corresponding to $130.4^{\circ}$. Although this bound is not very useful, we can limit the value of $d$ much more closely by comparing the experimental phase functions to the multiple-scattering phase functions in Fig. 6 . In each comparison, the same geometrical path-length difference has been subtracted from both theory and experiment. The closest match is clearly $d_{L}=1.30 \mathrm{~A}$. Because the angles $125.4^{\circ}$ and $127.5^{\circ}$ correspond respectively to $d_{L}=1.25 \AA$ and $d_{L}=$ 1.35 A, this comparison alone has the precision to set small error limits on $d_{\perp}$ (perhaps $\pm 0.02-0.03$ A).

We can also arrive at this conclusion by comparing the ARPEFS oscillations directly. Fig. 7 compares the filtered experimental data to theory curves for $S / N i$ bond lengths of $2.16 \mathrm{~A}\left(d_{L}=1.25 \AA\right), 2.19 \AA$ $(d,=1.30 \AA)$ and $2.22 \AA\left(d_{\perp}=1.35 \AA\right)$. Visual comparison is 
sufficient to exclude the two extreme geometries. Using the residual, an unweighted sum of the squared differences between experiment and theory, as a measure of the errors, we find a curve of error versus bond length whose minimum $l$ ies at $2.20 \mathrm{~A}(d=1.32 \mathrm{~A})$ as shown in Fig. 8 . The theoretical curves were first scaled to the experiment to minimize their residual before constructing the curve of errors to reduce the influence of amplitude factors.

This preference for 2.20 \& bond length is not dependent on the inner potential: the same geometry is found even if both theory and experiment are placed on the experimental energy scale and the inner potential of the theory is allowed to vary. The curve of errors is less sensitive to the structure in this case as can be seen in Fig. 8 because the shift in $E_{0}$ partly compensates for an incorrect geometry. At the minimum residual, we find an inner potential of $-11 \mathrm{eV}$, in good agreement with our original selection of $-10.5 \mathrm{ev}$. 
IV. DISCUSSION

Our measurement gives 2.20 \& for the $\mathrm{S}-\mathrm{Ni}$ bond length in c(2x2)S/Ni(001), in good agreement with previous studies. ${ }^{9}$ In estimating the precision of our determination we note that the structure information is carried in medium frequency oscillations superimposed upon a smoothly varying signal, and that we have sampled this signal at a much higher frequency than is relevant for the structure analysis. Furthermore we have measured the oscillations over a wide enough energy range to insure that errors in our reduction of the photoemission measurements to oscillations are minimal. The normal emission geometry is technically simpler to align and any small angular errors in the emission direction are self cancelling in the sense that among the four nearest neighbor $\mathrm{Ni}$ atoms every scattering path which lengthens with angle has a mate which shortens. We have varied the shape of the $I_{0}$ estinite by altering the stiffness of the numerical spline used to derived $I_{0}$ from the data without altering the backtransformed oscillations; the autoregressive Fourier transform is not essential for our analysis and the same results may be derived with conventional Fourier methods. A more complete discussion of possible errors may be found in ref 9. We believe our experimental precision is less than \pm $0.02 \AA$.

The accuracy of our bond length is unfortunately not entirely determined by experiment. Even though the Fourier filtering approach we have used here sufficiently restricts the theory problem so that we need not be concerned about convergence in multiple scattering order or curved wave corrections, our result still relies on accurate theoretical curves. By concentrating primarily on the frequency of the 
oscillations, our bond length is not sensitive to amplitude factors like inelastic mean free path, thermal averaging, or aperture integration. Furthermore, since the contribution of the path-length difference to the frequency is 20 times larger than the $\mathrm{Ni}$ potential phase shift and multiple scattering corrections, even moderate errors in the theoretical contributions to the frequency will not disturb the bond length analysis. More serious sources of error in our procedure are the constant part of the multiple scattering phase shift function and the inner potential, $E_{0}$. Either of these parameters will lead to geometry errors as the phase offset of theory and experiment is partial compensated by an erroneous shift in the theory path-length difference. Both the comparison of the curves in Fig. 8 and our residual analysis with variable $E_{0}$ in $F i g .9$ argue that we have made no large error due to constant phase shift or inner potential here. Altogether we estimate our accuracy as $\pm 0.02 \mathrm{~A}$ in bond length or $\pm 0.03 \mathrm{~A}$ in the $\mathrm{S}-\mathrm{Ni}$ interplanar spacing.

The theory errors are likely to be systematic, but we nevertheless quote our structure as $\mathrm{S}-\mathrm{Ni}$ bond length of $2.20 \AA \pm .03 \mathrm{~A}$ $(d=1.32 \pm .04 \mathrm{~A})$. With additional study of the scattering potential for $\mathrm{N} i$ and $\mathrm{S}$, including the photoion core potential, and additional measurements to insure experimental reproducibility, the accuracy of this type of structure measurement should improve by about a factor of two. We strongly emphasize that accurate structure work with extended fine structure requires a wide energy range. A short energy $r$ ange is subject to error from construction of the $x(k)$ curve to Fourier analysis to theory comparison: the additional data points in an extended range 
set the trends of the low frequencies and hence they provide more than a yimple statistical improvement in our experiment.

Sagurton, Bullock, and Fadley have recently ${ }^{17}$ studied the GRT resonance using single-scattering theory, and they have concluded trat the split Fourier peaks characteristic of the GRT resonance cannot be used for quantitative structural analyses. We believe that our work here demonstrates that this is not true, and rather than a serious liability, the GRT resonance has some interesting properties of its own. A more difficult problem is the contribution of double scattering to the frequency range occupied by the resonance, scattering which was omitted in the study of Sagurton et al. We have shown here that this problem can be overcome by applying the method of ref 2 .

Our goal in this study has been to study the surface geometry and the generalized Ramsauer Townsend resonance ultimately plays only a small role in our work. Understanding the GRT resonance is of course essential, but the presence of the resonance is a hindrance in the sense that the phase jump on resonance is sensitive to non-structural parameters. A minor benefit of the resonance is the relatively $f$ lat ( $k$ independent) nature of the $\mathrm{Ni}$ phase function above and below the resonance with a consequent negligible contribution to the oscillation frequency. We might imagine that the resonance itself could contribute to either the structure study in other surface systems or the study of surfaces in other ways. For example, first row adsorbates would scatter so little that direct comparison of the experimental phase function with the theory phase functions for various scattering angle might be sufficient to extract the structure. More intriguing, it may be possible to probe the electronic structure of the first layer of metal 
atoms in an adsorbate system by using the strong spin polarization ${ }^{18}$ which accompanies the GRT. This polarization has its origin in the sensitivity of the resonance: if the scattering potential has any dependence on spin, then the resonance energy will be spin dependent. At a photoelectron energy and scattering angle which corresponds to the GRT resonance, the photoemission intensity will be sensitive to the spin state of the scattering atom. 
v. CONCLUSION

We have observed a resonance in the scattering amplitude for $S(19)$ photoelectrons from $\mathrm{Ni}$ atoms and interpreted this resonance as a dip in the $\mathrm{Ni}$ atom partial cross section for electron scattering related to the Ramsauer-Townsend effect. This generalized Ramsauer-Townsend effect occurs at a particular energy and angle rather than in the total elastic cross section. We have shown that the resonance energy is sensitive to curved wave corrections and, after including some multiple scattering effects, we have derived the S-Ni bond length of $c(2 \times 2) S / N i(100)$ from the ARPEFS oscillations from nearest neighbor $\mathrm{Ni}$ atoms in the presence of the generalized Ramsauer-Townsend resonance. We $f$ ind this bond length to be $2.20 \AA \pm .03 \AA$ corresponding to a S-Ni interplanar distance $\left(d_{\perp}\right)$ of $1.32 \pm .04 \AA$. 


\section{REFERENCES}

1. P.J. Orders and C.S. Fadley, Phys. Rev, B 27, 781 (1983).

2. J.J. Barton, S.W. Robey, and D.A. Shirley, "Theory of AngleResolved Photoemission Extended Fine Structure", LBL-19324, and Chapter 5.

3. A. Liebsch, Phys. Rev. Lett. 32, 1203 (1974).

4. P.A. Lee, Phys. Rev. B 13, 5261 (1976).

5. D.A. Shirley, $C R$ in Solid State Materials Science 10, 373 (1982).

6. S.Y. Tong, and C.H. LI, in Chemistry and Physics of Solid Surface v. III, ed. R. Vanselow and W. England, CRC Press, (1982), p. 287.

7. J.J. Barton, C.C. Bahr, Z. Hussain, S.W. Robey, J.G. Tobin, L.E. Klebanoff, and D.A. Shirley, Phys. Rev. Lett. $\underline{51}, 272$ (1983).

8. J.J. Barton, S.W. Robey, C.C. Bahr, and D.A. Shirley, presented at the First International Conference on the Structure of Surfaces, Berkeley, CA., to be published in the Springer Series in Chemical Physics, 1985.

9. J.J. Barton, C.C. Bahr, S.W. Robey, Z. Hussain, and D.A. Shirley, "The Measurement and Analysis of ARPEFS Data: Application to c(2X2)S/Ni(001)",LBL-14993, Chapter 7 , ana references therein.

10. Z. Hussain, E. Umbach, D.A. Shirley, J. Stohr, and J. Feldhaus, Nucl. Instrum. Methods 195, 115 (1982).

11. J.J.Barton and D.A. Shirley, "Fourier Analysis of Extended Fine Structure with Autoregressive Prediction", LBL-14758, and Chapter 6.

12. H. Faxen and J. Holtsmark, Z. Physik 45, 307 (1927).

13. For some elements the generalized Ramsauer Townsend effect occurs between $3 \mathrm{~A}^{-1}$ and $12 \mathrm{~A}^{-1}$ at $180^{\circ}$. It then appears in the EXAFS 
spectrum. B.K. Teo and P.A. Lee, J. Am. Chem. Soc. 101, 2815 (1979).

14. G. Martens, P. Rabe, N. Schwentner, and A. Werner, Phys. Rev. B 17, $1481(1978)$.

15. J.J. Barton, C.C. Bahr, Z. Hussain, S.W. Robey, L.E. Klebanoff, and D.A. Shirley, Proceedings for the Brookhaven Conference, Advances in Soft X-ray Science and Technology, S.P.I.E. v. 447, 82 (1984).

16. J.J. Barton and D.A. Shirley, "Curved Wavefront Corrections for Photoelectron Scattering", LBL-18692, submitted to Phys. Rev. $B$, and Chapter 2.

17. M. Sagurton, E.L. Bullock, and C.S. Fadley, Phys. Rev. B 30, 7332 (1984).

18. H.S.W. Massey, E.H.S. Burhop, and H.B. Gilbody, Electronic and Ionic Impact Phenomena, v. 1, 2nd ed., Oxford Univ. Press, London (1969). 
FIGURE CAPTIONS

Figure 1.

$S(1 s)$ ARPEFS oscillations from $c(2 \times 2) \mathrm{S} / \mathrm{Ni}(001)$ in normal emission ([001]). The experimental kinetic energy scale has been converted to a wavenumber scale using an inner potential of $10.5 \mathrm{eV}$, and the resulting curve has been interpolated with a numerical spline to an even mesh of 128 points.

Figure 2. Fourier transform magnitudes versus scattering pathlength difference for $k$ times the data in Fig. 1. In the lower panel, the conventional Fourier transform was applied, while the upper panel was obtained with the autoregressive linear prediction method described in ref. 11.

Figure 3. Ni scattering amplitudes calculated in the plane wave limit. Each solid line represents the scattering amplitude for the scattering angle indicated. For each scattering angle the amplitude was calculated for wavenumbers from 5-12月 ${ }^{-1}$; the labeled tick marks give some indication of the wavenumber scale. The amplitudes are plotted in the complex plane to illustrate the connection between scattering intensity and phase shift. Note that the scattering intensity is nearly zero for $\theta_{j}=130^{\circ}$ and $k=8 \mathrm{~A}^{-1}$. 
Figure 4.

Fourier filtered ARPEFS data. The Fourier spectrum from EIg. 2 was zeroed above $5.1 \AA$ and backtransformed, the amplitude envelop is also plotted as obtained from the complex backtransformation.

Figure 5. Phase shifts for scattering from $\mathrm{Ni}$. The dashed line shows the phase shift calculated with plane wave theory $\theta_{j}=127^{\circ}$. The dotted line is the phase shift from the experimental curve Fig. 2, where the first two Fourier peaks are backtransformed together. A factor of $\pi$ for the sign difference between direct and scattered waves caused by the $p$ wave angular distribution has been added to the experimental phase and a nominal $3.56 \mathrm{~A}$ path-length difference has been removed. The zero crossing jump in phase occurs too high in wavenumber for the plane wave calculation. Solid lines are curved-wave calculations of the phase shift for the indicated scattering angles.

Figure 6. Multiple-scattering GRT phase jumps. Each panel is labeled by the scattering angle for the $\mathrm{Ni}$ nearest neighbors. The dashed lines are phase jumps from the scattering calculation described in the text. The solid lines with circles are experimental phase jumps with geometrical path lengths removed. A phase equal to $3.57 \mathrm{k}$ radians was subtracted from both theory and experiment phase functions for comparing the $127.5^{\circ}\left(d_{\perp}=1.35 \AA\right)$ phases, $3.49 \mathrm{k}$ radians from $126.4^{\circ}\left(\mathrm{d}_{\perp}=1.30 \AA\right)$, and $3.41 \mathrm{k}$ radians from $125.4^{\circ}\left(d_{\perp}=1.25 \AA\right)$. As in Fig. 5, a factor 
of $\pi$ for $(-1)$ was added to all phase functions; an additional factor of $\pi$ was added to the $131.5^{\circ}$ phase for the purpose of display.

Figure 7 . Numerical simulation of the normal emission, S(1s) ARPEFS from $c(2 \times 2) \mathrm{S} / \mathrm{Ni}(001)$ compared to Fourier filtered experimental data. The theory curves were calculated for all scattering paths less than $5.1 \mathrm{~A}$; the experimental AR Fourier transform in Fig. 2 was zeroed for frequencies above $5.1 \AA$ and backtransformed to give the solid sircles. Over the entire energy range, the frequency of the oscillations clearly matches the theory curve for a S-Ni bond length of 2.19 A better than the curves for shorter or longer bond lengths.

Figure 8. Geometry search for S-Ni interlayer spacing. Plotted symbols are residuals from the least-squares fit of the numerical simulation curves to the Fourier filtered data. The residual is the unweighted sum of the squared differences between theory and experiment between 100 and $414 \mathrm{eV}$. The solid triangles give the residual for a fixed theory inner potential of $10.5 \mathrm{eV}$; the crosses correspond to fits in which the theory inner potential was varied. The solid curve is a parabolic fit to the four triangle points between 1.275 and $1.35 \mathrm{~A}$; the dashed curve is a parabolic fit to the four crosses in the same region. 


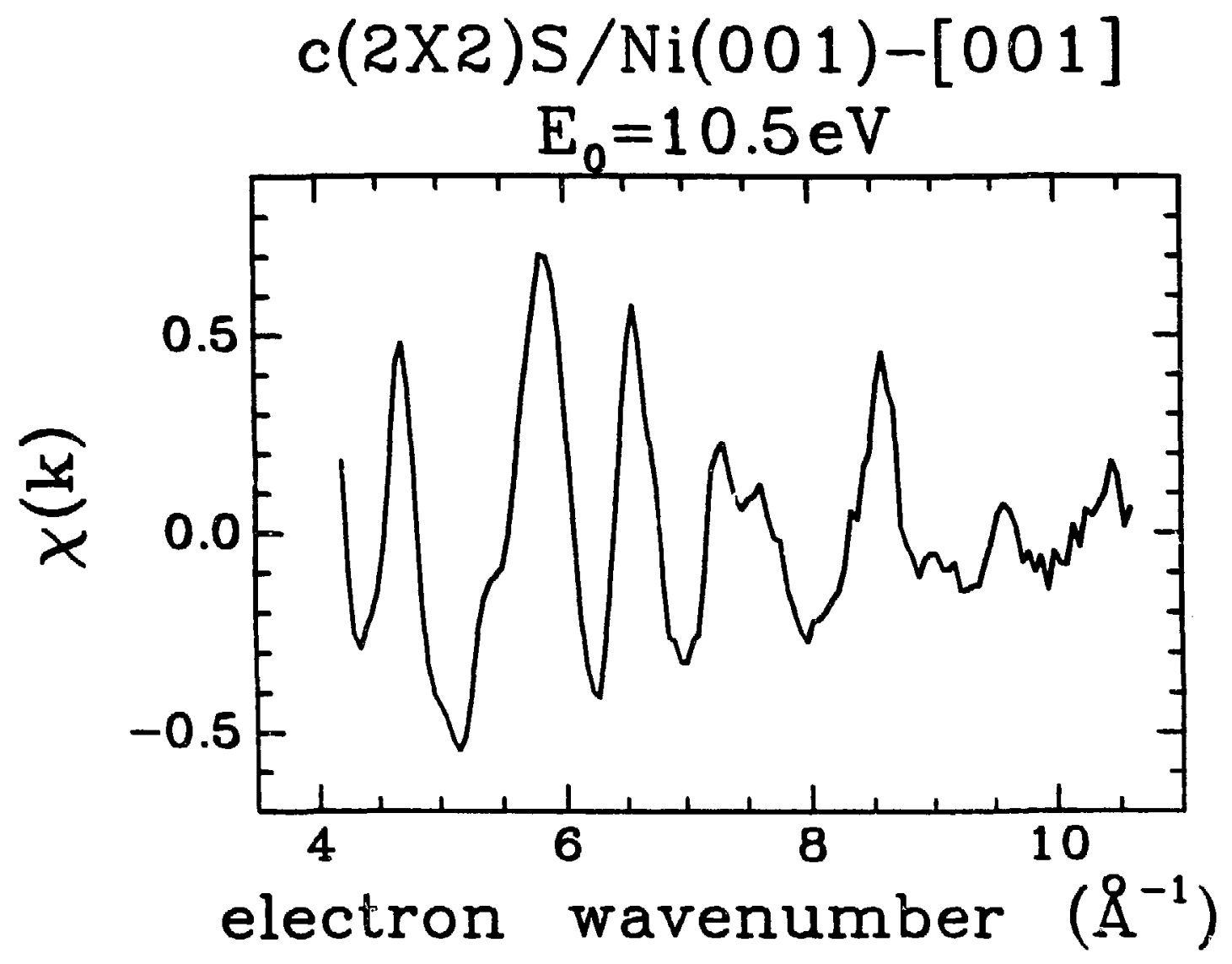

Figure 1 
$\mathrm{c}(2 \times 2) \mathrm{S} / \mathrm{Ni}(001)-[001]$

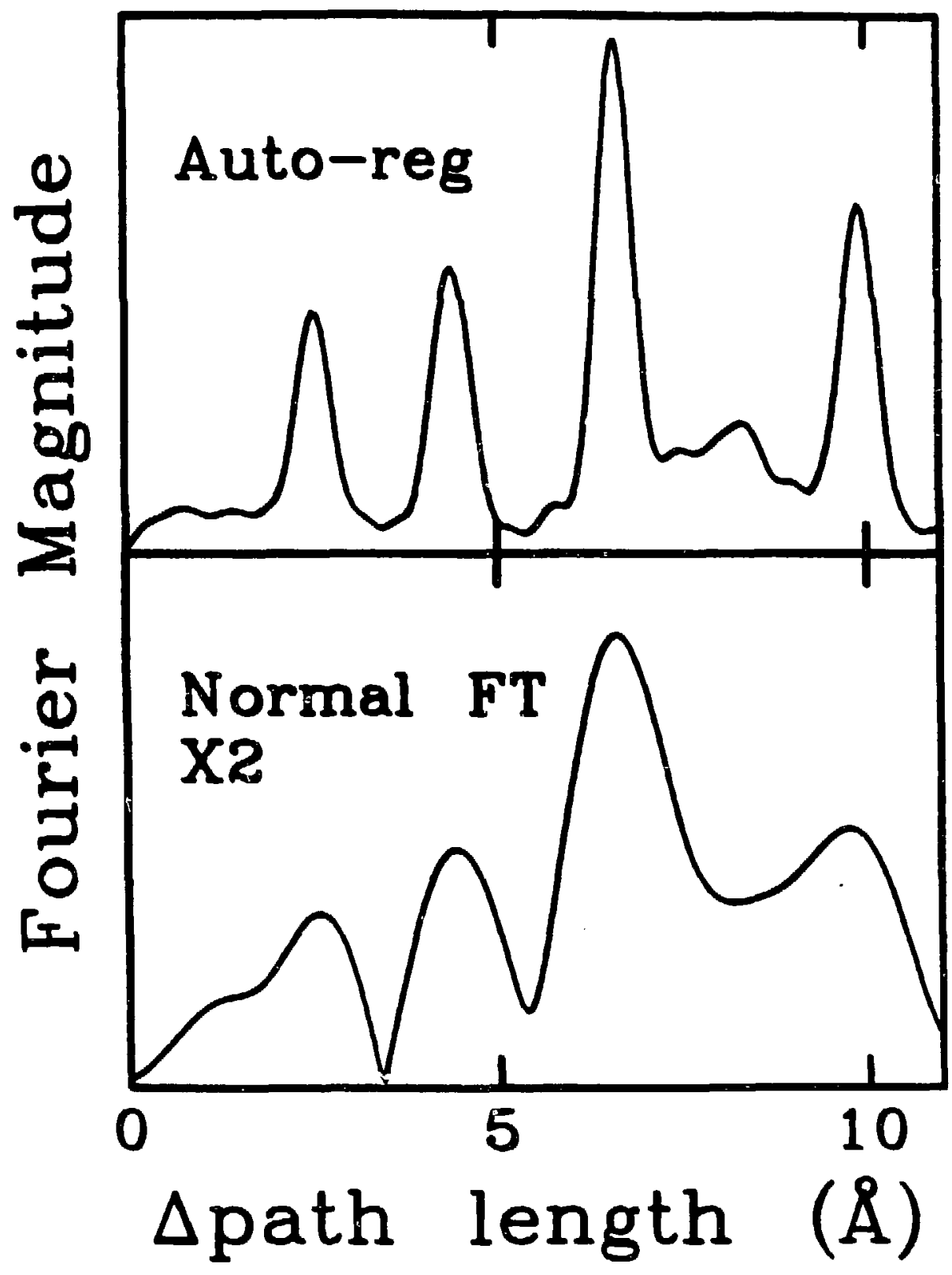

XBL 853-1836

Figure 2 


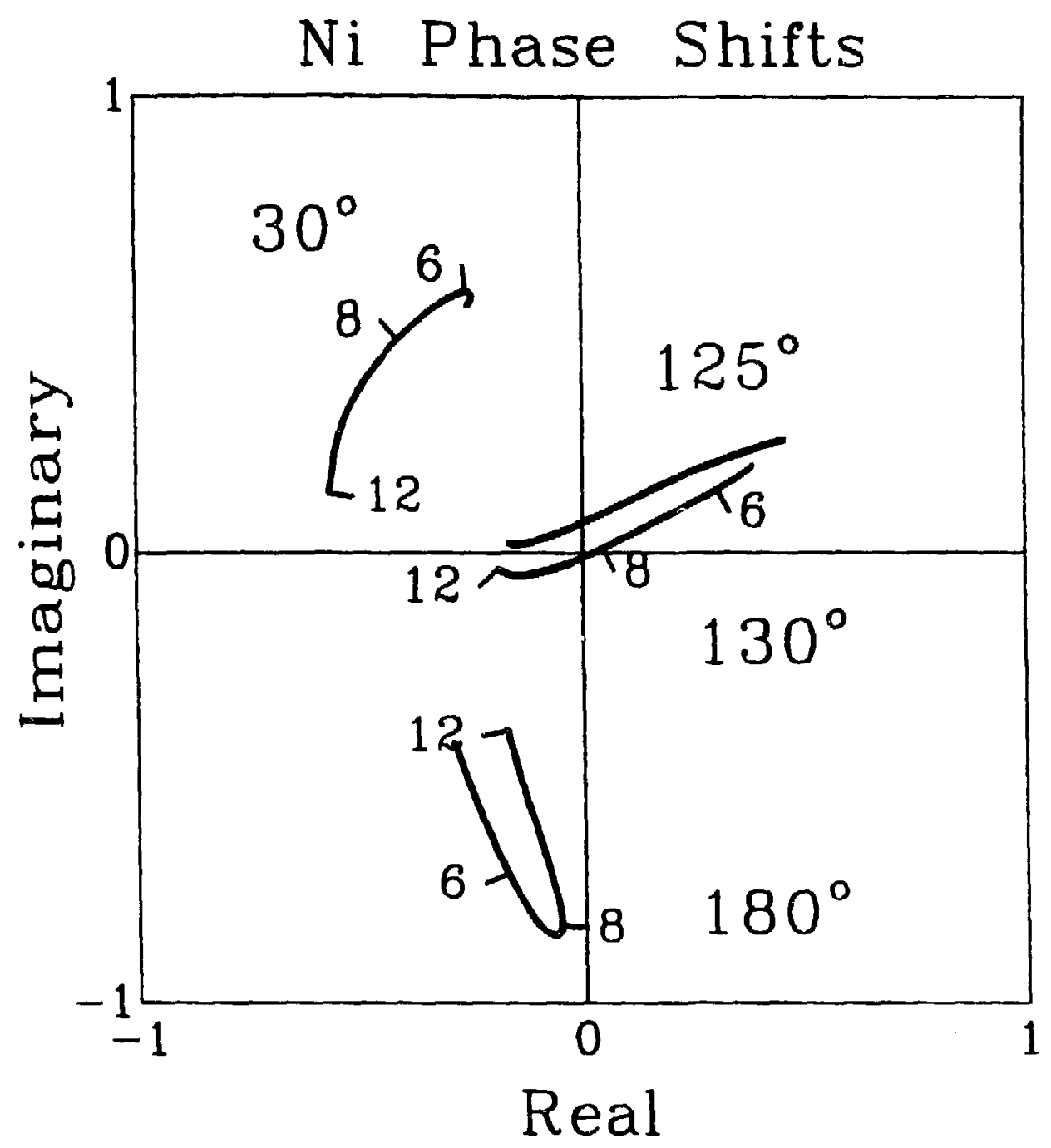

XBL 839-11333

Figure 3 


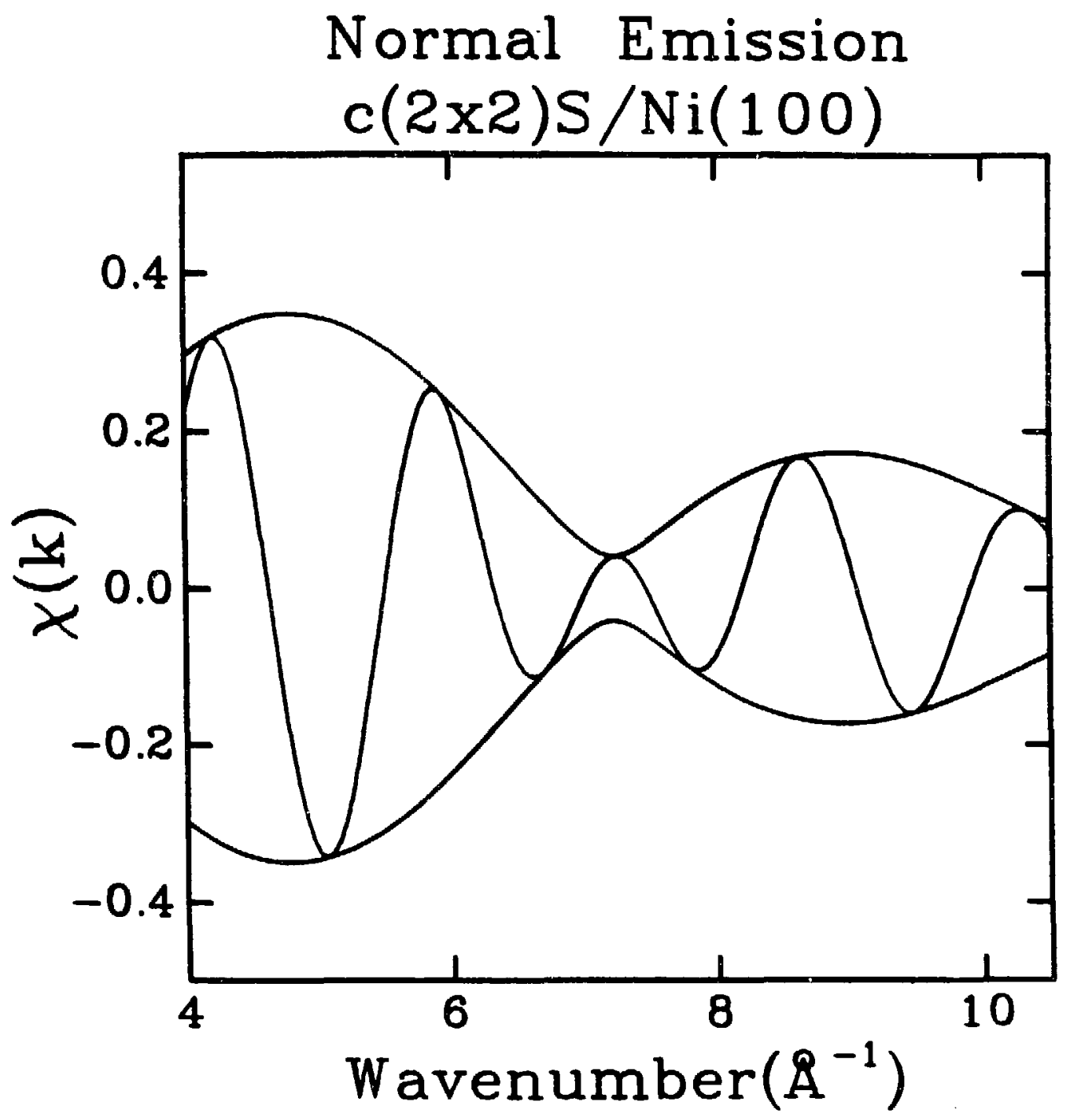

XBL $853-1840$

Figure /4 


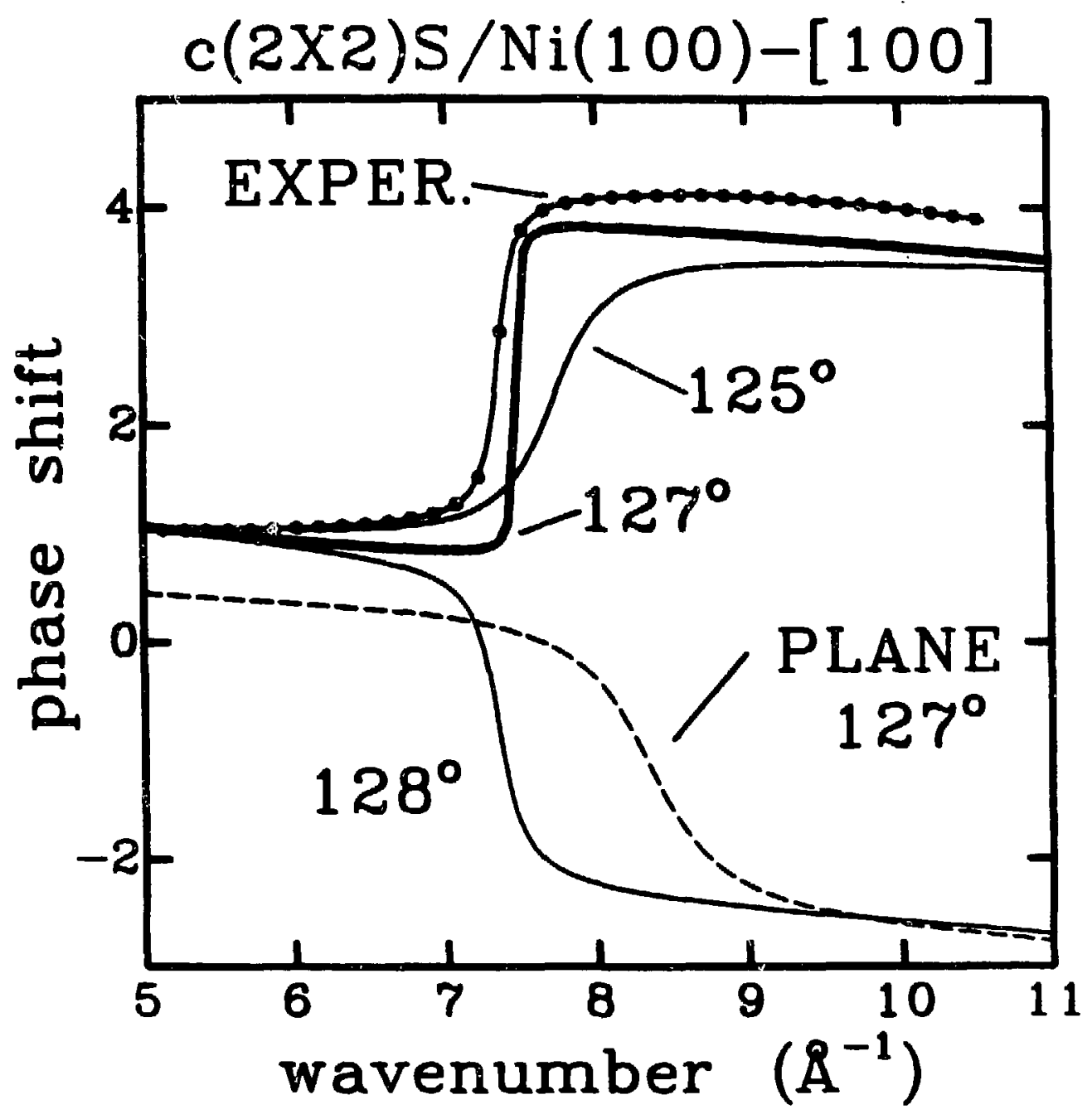

DK:NIS1 11 DUS

XBL 848-3548

Figure 5 


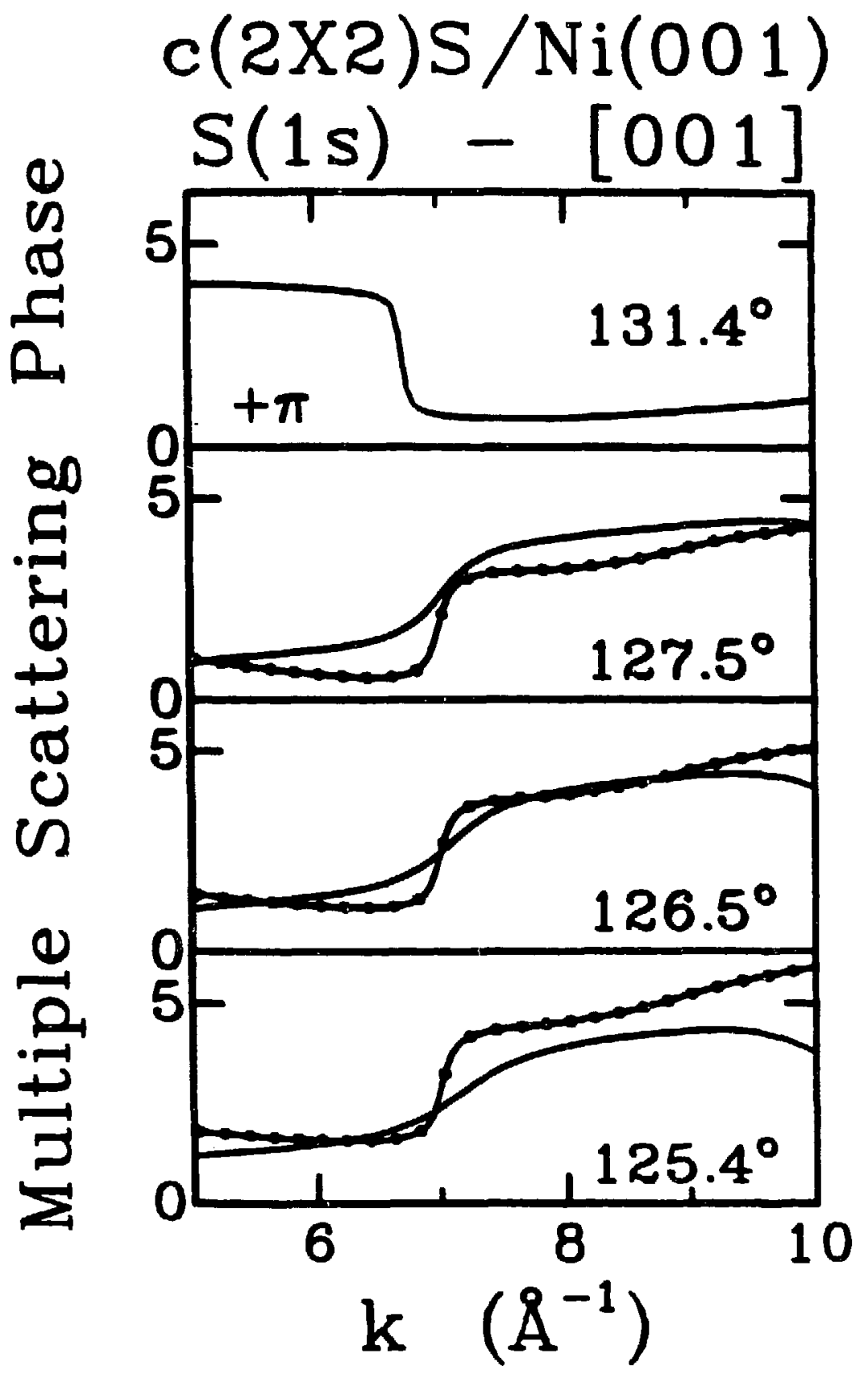

XBL 853-1830

Figure 6 


\section{c(2X2)S/Ni(001)-[001] 0-5\& Backtransform}

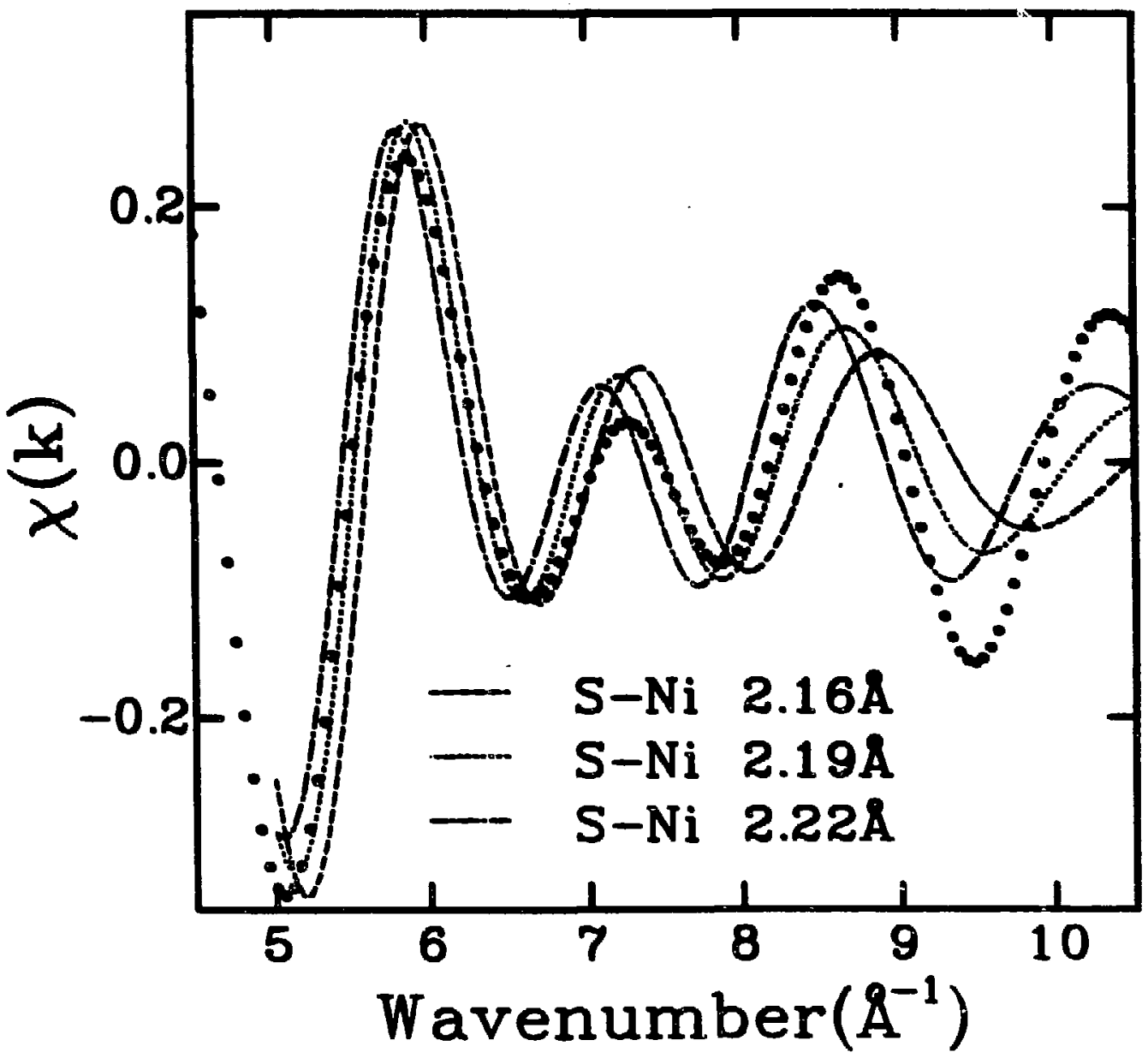

XBL $853-1826$

Figure 7 


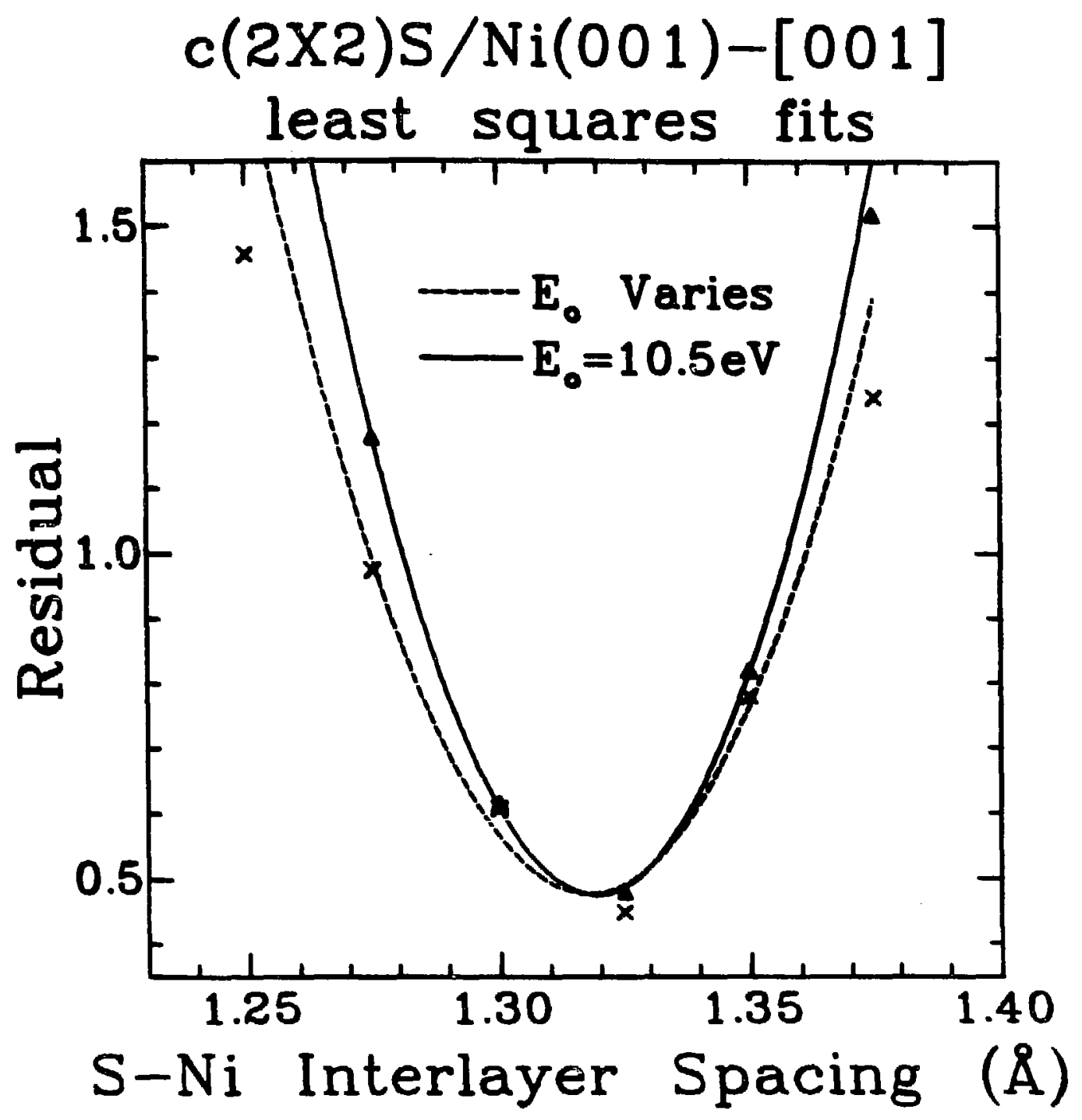

XBL 853-1827 


\section{CHAPTER 9:}

\section{CONCLUSION}

The preceding chapters have drawn specific conclusions on the particular topics they discuss, and they each contain their own summary. Thus we will use this conclusion to suggest what new directions may be taken based on the work presented here. We begin with a broad view and follow with suggestions relating to the individual chapters.

We might first ask if, based on our current progress, the method determining surface structures from photoelectron partial cross-section measurements merits further work. The key features of ARPEFS which recommend it for structure work are:

1) Chemical Specificity: The structural signal is contained in core-level partial cross-section oscillations. By selecting the core level observed, we select the element or even oxidation state of an element to study.

1i) Surface Sensitivity: Using photoelectrons in the 100-500 eV energy range gives good surface sensitivity.

111) Large Oscillation Amplitude: The detected interference is between direct and scattered waves, giving typical oscillations of 20-50\%.

1v) High Angular Sensitivity: Each different emission direction ylelds a different view of the structure; each different combination of polarization direction and crystal orientation gives different emphasis to the scattering atoms. 
v) Simple Theoretical Model: The above four experimental considerations combine to greatly simplify curved-wave, multiple-scattering calculations.

vi) Direct Fourier Analysis: The Fourier transform amplitude maps out scattering power versus geometrical path-length difference. The Fourier transform provides a means of displaying the structure information directly from a measurement.

vii) High Precision: The experimental curves can be measured and reduced to infer a structure in a fashion which would not Iimit the accuracy of the structure.

We must balance these advantages against the drawbacks of structure determination with ARPEFS and against alternative techniques of equal ability but greater ease. The two greatest barriers to the use of ARPEFS as a structure technique are the use of synchrotron radiation and the reliance on theoretical values for the inner potential and scattering phase functions. Synchrotron radiation beam time is currently very limited, but the future looks very bright. New electron storage rings dedicated to producing synchrotron radiation are opening throughout the world, and these new racilities provide more $x$-rays over a wider energy range than we have avallable today. Equally important, new designs for the experimental apparatus used on these storage rings will allow much more effective use of the avallable beam time.

The problem of theoretical parameters is a difficult one but one which should not be unsurmountable. On the scale of modern methods for electronic structure calculations, our scattering phase functions are not very sophisticated. On the other hand, ARPEFS measurements can 
produce such a large amount of information about the scattering of electrons that we may be able to devise a purely empirical set of scattering phase functions and eliminate any concern for the quality of the theory. The determination of the inner potential is tractable by some experimental method, but a deeper understanding of intermediate velocity electron propagation in solids is certainly required. The inner poten'ial problem, electron refraction, inelastic electron scattering, and dynamic screening of the photoelectron and core hole are interesting interconnected problems which should be attacked both theoretically and experimentally.

With these considerations, we briefly recall the advantage of the two most used structure methods: Low Energy Electron Diffraction (LEED) and Surface Extended X-Ray Absorption Fine Structure (SEXAFS).

When comparing ARPEFS to other structure techniques we must recall that ARPEFS measures a differential cross section: for its advantages to be manifest the sample must be orientationally ordered. That is, the vectors from the photoemitter to each scattering atom must be the same for all emitting atoms. (We could, of course, have a few distinct emitting atom locations, but not hundreds.) often this is equivalent to the periodic ordering required for LEED, but there may be cases in which this distinction is important. One example is the conversion between LEED patterns where we might expect orlentational ordering, but the twodimensional periodicity is not complete. For a randomly oriented system, an ARPEFS measurement should be equivalent to a surface EXAFS measurement, but one may also be interested in the ARPEFS measurement of a disordered system: presumably with some effort a theory of 
orientational disorder could be verifled with experimental ARPEFS measur ements .

LEED continues to make steady progress. The advent of high gain detectors reduces the electron beam damage effects that previously limited this technique. Theoretical refinements have improved the prospects for solving more complicated structures. The major strength of LEED--experimental simplicity--must be balanced against the complexity of its analysis. Clearly the large number of simple structures solved by LEED suggests that for these systems the complexity is tractable.

Surface EXAFS has made giant strides since its introduction. In return for venturing to a synchrotron storage ring, SEXAFS provides direct structure analysis including highly accurate bond lengths without requiring an ordered overlayer structure. The key advantage of SEXAFS-direct measurement of an average bond length to nearest neighbors--is also its key weakness for complicated structures. A well characterized but complex adsorbate could have many similar bond lengths which SEXAFS cannot resolve. For disordered systems, however, the average bond length may be the only useful parameter to describe the structure, and SEXAFS would be uniquely suitable.

Rather than emphasize the competition of these structure techniques we should recognize their complementary nature. ARPEFS exhibits some of the features of both LEED and SEXAFS and in combination with these techniques perhaps we can build a collection of reliable structures essential for an understanding of chemical bonding on surface. We have demonstrated that ARPEFS can solve simple surface structures such as the $c(2 \times 2) S / N i(001)$ structure discussed in this 
thesis. Atomic systems which will benefit from the advantages of ARPEFS Include:

1) face-centered cubic (111) surfaces where two different threefold hollow sites have similar nearest nelghbor distances but are distinguished by second layer substrate atoms. Normal emission ARPEFS would be ideal for scattering from the second layer atom.

11) Multiple site adsorption. More than one occupied site will likely have similar bond lengths but very different pathlength differences in some directions.

1ii) Atoms on stepped surfaces. Here the high angle selectivity would be valuable to concentrate on only the atoms on the edges.

Beyond atomic systems, the more complicated the adsorbate molecule, the more advantages of ARPEFS will be evident. ARPEFS can dissect a molecular adsorbate structure problem by examining each element from each of several different view points. With direct analysis and adequate development of the electron scattering theory, ARPEFS is capable of determining these structures.

Each of the chapters in this thesis covered topics which suggest important directions for future work. Some of these ideas are covered here so that we may finish with a look forward.

The lifting operator or derivative expansion approach to electron scattering in Chapter 2 has a great many applications toward practical improvement of the theory of electron scattering. Because the higher order curved-wave corrections appear as derivatives of the basic homogeneous wave scattering, other types of corrections can be written 
in terms of the same elements already available in the treatment of Chapter 2. The expressions for thermal averaging of the scattering amplitude derived in Chapter 5 is one example. Another interesting direction is automatic, gradient-driven, geometry searches. The error surfaces plotted in Chapter 6 are superfluous to the final result: we are only interested in the minimum point and possibly the curvature of the error surface. The geometry terms enter the expression for the least-squares error in a stralghtforward fashion, and formulas for the gradients and curvatures of the error surface could be derived in terms of the sattering factors from Chapter 2 with only moderate effort, leading to a quadratically convergent geometry optimization method. One of the prime motivations for the work of Chapters 3 and 4 was the difficulty in obtaining second order curved-wave corrections with the lifting operator method. Having completed example calculations with the Taylor Series Magnetic Quantum Number Expansion method, we now know that second order corrections are small, and the first order derivatives from Chapter 2 could be used successfully for most problems. Finally, it might be useful to elaborate the connection between the lifting operator and TS-MQNE formulae to facilitate conversion between them as a practical problem might require.

The approximate origin-shift addition theorem derived in chapter 3 is merely a tool for the development of the electron scattering formulae, but the magnetic quantum number expansion gives us a tangible physical picture for the nature of the approximation. Unfortunately, this addresses only one of the two expansion variables, and a more thorough understanding of the Fourier transform or rotation matrix concepts may allow us to "see" the other direction as well. Beyond 
pleasing our curiosity, we notice that the radial correction terms from Chapter 2 to which this other expansion variable corresponds are usually small: If we understood this variable, we may be able to truncate the expansion to which it corresponds or even eliminate it in many cases. Another direction to explore with the origin-shift equations is the expansion of Coulomb waves as would be appropriate for non-metal substrates and exponentially damped spherical Hankel waves which more accurately characterized the nature of spherical waves in a metal.

The small atom approximation ideas and in particular the Taylor Series Magnetic Quantum Number Expansion formulae from Chapter 3 should be directly applicable to other electron scattering prob: ers, such as EXAFS, LEED, and inelastic electron spectroscopies. Whe he' this application would represent a practical improvement could only be judged by trial. As the rotation of the coordinate system makes symmetry very difficult to incorporate, the TS-MQNE approach may be more valuable for problems with low symmetry where special methods do not apply. The properties of the scattering factors deserve another look since their number determines the cost of the calculation. In particular it may be possible to generate some of these factors from simple operations on the others, a great savings when the number of partial waves is large. of more lmmediate use would be formulae for $p, d$, and $f$ initial core-level ARPEFS, but this should be primarily a matter of computer implementation since the electron scattering equations do not change. This is in contrast to the lifting operator formulae of Chapter 2 where derivatives of the higher order waves are required.

The theory of ARPEFS presented in Chapter 5 is barely adequate to allow surface structure determination, and the discussion of many points 
brought out more problems than solutions. Foremost among the theoretical challenges is the possibility as systematic error in the scattering partial wave phase shifts. By no means should our efforts at simulating the experiment be considered as good as can be done. The scattering partial wave phase shifts we used, while comparable to others used in this energy range, are based on old 1deas about electronic structure which have not benefited from two decades of research in this field. All the avallable evidence suggests that the scattering potentials in the intermediate energy range should not be very sensitive to chemical bonding so that once a potential was demonstrated to be adequate for, say, $\mathrm{N} 1$ metal, it would be useful for all subsequent studies on $\mathrm{Ni}$. We have also neglected the photoion core in Chapter 5 , and any study of potentials should include the core-hole effect. A more satisfactory treatment of electron-electron interaction is also required to understand the inner potential and refraction problems; equally important, the adequacy of an isotropic mean free path should be reinvestigated. Our development was primarily aimed at metallic systems like S/N1, so a thorough re-examination of many points is required before studying semiconductors and lonic solids. Another rough spot is the Debye model for thermal averaging. From the experimental viewpoint the Debye model is adequate as it provides a convenient parameterization which is correct at high and low temperatures and smooth between. This suggests that a more fundamentaliy satisfactory formulation with the same properties may be avallable, perhaps based on the frequency distribution moment expansion. Finally, a careful comparison of our results and method to full multiple scattering, LEED-like theories is in order to understand the range of applicabllity of our approach. 
Fourier transformation of the energy dependent photoelectron diffraction and the subsequent interpretation of the Fourier spectra were dramatic developments in the use of photoelectron diffraction for surface structure analysis. Chapter 6 grapples with the technological aspects of the Fourier transform and while the resuits are not perfect, insight gained from relatively simple manipulation of the data cannot be denied. Whenever some aspect of a frequency spectrum does not agree with a preconcelved idea, it is a signal that one's entire knowledge about a problem has not been transmitted to the data analysis. Thus conventional Fourier analysle has low resolution because the information that we have a finite length estimate of a hypothetically infinitely long slgnal was not included. Similarly autoregressive Fourler analysis gives split peaks because the information that our signal has amplitude structure was not included. Another approach to Fourier analysis may develop whish can account for at least part of the amplitude dependence in ARPEFS; before the advent of the autoregressive algorithms, one might have concluded only conventional Fourier methods and least-squares fitting were possible. For example, one may be able to exploit the connection between the constant phase shift largely determined by the scattering phase and the scattering amplitude to deduce the scattering envelope. Along these lines one must imagine a three-dimensional transformation in which ARPEFS spectra from many different emission directions are processed directly into a three-dimensional image of the surface structure in a manner somewhat analogous to $x$-ray tomography recons tructions.

The experimental work in Chapter 7 amounts to a feasibility study for measuring surface structures with ARPEFS and despite some probiems 
the results are very favorable. Thus foremost among new experimental directions is the measurement of ARPEFS from other surface systems. Concurrent with this thesis more than a dozen measurements have been made by other members of our research group, and they are currently being analyzed for structure. In addition to studies of surface structure, a temperature dependent ARPEFS measurement would test the thermal averaging theory as well as contributing to the understanding of vibrations on surfaces. New experimental apparatus would dramatically improve the measurements of ARPEFS. Two designs in particular currently under construction in our laboratory will increase the precision and the number of measured curves. The first improvement is a new type of crystal goniometer in which the vacuum rotation is accomplished with sliding seals rather than rotating bellows. This difference is very signiflcant because the sliding seal arrangement allows trie : inple to be mounted on the end of a stiff sealed tube: the position of the sample can be maintained to $<0.01^{\circ}$. The second improvement is a new type of electron energy analyzer incorporating a focusing parabololdal electrostatic mirror, a time-of-flight analyzer, and a position encoding detector. The parabololdal electron mirror has -100 times larger angular acceptance than the apparatus used for the experiments in this thesis, and the time-of-flight technique should be several times more efficient in measuring photoemission spectra. The position encodingdetector means that several tens of simultaneous ARPEFS spectra can be accumulated, leading to a high probability of a definitive structure analysis after a single sweep of the photon energy. Other experimental directions should explore alternative sources for creating ARPEFS-like sigmals. Electron energy loss with or without coincidence is the first 
candidate, but two other possibilities can be suggested. First, the Einstein relation, $E_{k}=h v-E_{B}$, connects every photon energy with only one electron kinetic energy. Thus, in principle one does not require a photon monochromator for ARPEFS measurements. It should suffice to place the sample in a broadband $x$-ray source and scan the electron energy as long as the background signal is not too large. Second, a similar broadband source is in principle available in $\beta$ decay of nuclei. Thus the $\mathrm{e}^{-}$ejected from tritium should show ARPEFS oscillations, giving one of the few ways to study the structure of such a light element on surfaces.

Finally we come to Chapter 9. A catalog of the positions in energy and angle of the Generalized Ramsauer-Townsend resonances for the period chart is a necessary adjunct to surface structure work, and the trends In the resonance positions with atomic number should lead to a deeper understanding of the physical origins of these resonances. We have noted in Chapter 9 that one may be able to exploit the spin dependence of the resonance position, but the resonance is also related to the time dependence of the scattering event. Near resonance, the electron is delayed in the region of the scattering atom, and one might devise some way to exploit this delay to study the dynamics of photoabsorption.

Hopefully this thesis demonstrates that surface structure determination with photoelectron diffraction and, in particular, the angle-resolved photoemission extended fine structure is on the verge of fulfilling its early promise as a general purpose method for measuring surface structures. With additional development, ARPEFS analysis should contribute to our knowledge of the structure of surfaces and ultimately to our understanding of surface chemistry. 


\section{ACKNOWLEDGEMENTS}

This work was supported by the Director, Office of Energy Research, Office of Basic Energy Sciences, Chemical Sciences Division of the U.S. Department of Energy under Contract No. DE-AC03-76SF00098. It was performed at the Stanford Synchrotron Radiation Laboratory, which is supported by the Department of Energy, Office of Basic Energy Sciences and the National Science Foundation, Division of Materials Research. 
This report was done with support from the Department of Energy. Any conclusions or opinions expressed in this report represent solely those of the author(s) and not necessarily those of The Regents of the University of California, the Lawrence Berkcley Laboratory or the Department of Energy.

Reference to a company or product name does not imply approvai or recommendation of the product by the University of Califomia or the U.S. Department of Energy to the exclusion of others that may be suitable. 